\title{
Progress and Perspectives of Electrochemical CO2 Reduction on Copper in Aqueous Electrolyte
}

Nitopi, Stephanie A.; Bertheussen, Erlend; Scott, Søren Bertelsen; Liu, Xinyan; Engstfeld, Albert Kilian; Horch, Sebastian; Seger, Brian; Stephens, Ifan; Chan, Karen; Hahn, Christopher

Total number of authors:

13

Published in:

Chemical Reviews

Link to article, DOI:

10.1021/acs.chemrev.8b00705

Publication date:

2019

Document Version

Peer reviewed version

Link back to DTU Orbit

Citation (APA):

Nitopi, S. A., Bertheussen, E., Scott, S. B., Liu, X., Engstfeld, A. K., Horch, S., Seger, B., Stephens, I., Chan, K., Hahn, C., Nørskov, J. K., Jaramillo, T., \& Chorkendorff, I. (2019). Progress and Perspectives of Electrochemical $\mathrm{CO}_{2}$ Reduction on Copper in Aqueous Electrolyte. Chemical Reviews, 119(12), 7610-7672.

https://doi.org/10.1021/acs.chemrev.8b00705

\section{General rights}

Copyright and moral rights for the publications made accessible in the public portal are retained by the authors and/or other copyright owners and it is a condition of accessing publications that users recognise and abide by the legal requirements associated with these rights.

- Users may download and print one copy of any publication from the public portal for the purpose of private study or research.

- You may not further distribute the material or use it for any profit-making activity or commercial gain

- You may freely distribute the URL identifying the publication in the public portal 


\section{Progress and Perspectives of Electrochemical $\mathrm{CO}_{2}$ Reduction on Copper in Aqueous Electrolyte}

Stephanie A. Nitopi, ${ }^{\mathrm{a}, \sim}$ Erlend Bertheussen, ${ }^{\mathrm{b}, \sim}$ Soren B. Scott, ${ }^{\mathrm{b}}$ Xinyan Liu, ${ }^{\mathrm{a}}$ Albert K. Engstfeld, ${ }^{\text {b,c }}$ Sebastian Horch, ${ }^{b}$ Brian Seger, ${ }^{b}$ Ifan E.L. Stephens, ${ }^{\text {be }}$ Karen Chan, ${ }^{\text {a,d }}$ Christopher Hahn, ${ }^{\text {a,d }}$ Jens K. Nørskov, ${ }^{\text {a,b,d,* }}$ Thomas F. Jaramillo, ${ }^{\text {a,d,* }}$ and Ib Chorkendorff ${ }^{b, *}$

${ }^{\text {a }}$ SUNCAT Center for Interface Science and Catalysis, Department of Chemical Engineering, Stanford University, Stanford, California 94305, United States

${ }^{\mathrm{b}}$ Section for Surface Physics and Catalysis, Department of Physics, Technical University of Denmark, 2800 Kgs. Lyngby, Denmark

${ }^{\mathrm{c}}$ Institute of Surface Chemistry and Catalysis, Ulm University, D-89069 Ulm, Germany

${ }^{d}$ SUNCAT Center for Interface Science and Catalysis, SLAC National Accelerator Laboratory, Menlo Park, California 94025, United States

${ }^{\mathrm{e}}$ Department of Materials, Imperial College London, Royal School of Mines, London SW7 2AZ, U.K.

These authors contributed equally to this work.

*Corresponding authors: ibchork@fysik.dtu.dk, jaramillo@stanford.edu, jkno@dtu.dk

\section{Abstract}

To date, copper is the only heterogeneous catalyst that has shown a propensity to produce valuable hydrocarbons and alcohols, such as ethylene and ethanol, from electrochemical $\mathrm{CO}_{2}$ reduction $\left(\mathrm{CO}_{2} \mathrm{R}\right)$. There are variety of factors that impact $\mathrm{CO}_{2} \mathrm{R}$ activity and selectivity, including the catalyst surface structure, morphology, composition, the choice of electrolyte ions and $\mathrm{pH}$, the electrochemical cell design, etc. Many of these factors are often intertwined, which can complicate catalyst discovery and design efforts. Here we take a broad and historical view of these different aspects and their complex interplay in $\mathrm{CO}_{2} \mathrm{R}$ catalysis on $\mathrm{Cu}$, with the purpose of providing new insights, critical evaluations, and guidance to the field with regards to research directions and best practices. First, we describe the various experimental probes and complementary theoretical methods that have been used to discern the mechanisms by which products are formed, and next we present our current understanding of the complex reaction networks for $\mathrm{CO}_{2} \mathrm{R}$ on $\mathrm{Cu}$. Then we analyze two key methods that have been used in attempts to alter the activity and selectivity of $\mathrm{Cu}$ : nanostructuring and the formation of bimetallic electrodes. Finally, we offer some perspectives on the future outlook for electrochemical $\mathrm{CO}_{2} \mathrm{R}$. 


\section{Table of Contents}

1. Introduction

1.1. Motivation for $\mathrm{CO}_{2}$ Recycling

1.1.1. Closing the Carbon Cycle

1.1.2. Strategies for $\mathrm{CO}_{2}$ Recycling

1.2. Electrochemical $\mathrm{CO}_{2}$ Reduction

1.2.1. Desired Products: Thermodynamics and Economics

1.2.2. Copper as a Unique Electrocatalyst

2. Considerations for Conducting \& Comparing Electrochemical $\mathrm{CO}_{2}$ Reduction Experiments

2.1. Reporting Data

2.2. Intrinsic Kinetics vs. Mass Transport Effects

2.3. Deactivation of $\mathrm{Cu}$ Electrodes

2.4. Deconvolution of Various Effects in Complex Catalysts

3. Experimental Probes of $\mathrm{CO}_{2}$ Reduction Mechanisms

3.1. The Influence of Surface Facet on $\mathrm{CO}_{2}$ and $\mathrm{CO}$ Reduction

3.1.1. Influence of Various $\mathrm{Cu}(\mathrm{hkl})$ Surfaces on Product Distribution

3.1.2. Influence of Various $\mathrm{Cu}(\mathrm{hkl})$ Surfaces on Activity

3.1.3. Restructuring and Other Challenges Regarding Single Crystal Measurements

3.2. Reduction of Possible Intermediates

3.2.1. $\mathrm{CO}$ as a Crucial Intermediate

3.2.2. Reduction of Oxygenated $\mathrm{C}_{1}$ and $\mathrm{C}_{2}$ Species

3.3. Electrolyte Effects

3.3.1. pH Effects

3.3.2. Cation Effects

3.3.3. Anion Effects

3.4. Electrochemical Conditions

3.4.1. Temperature \& Pressure

3.5. Summary

4. Theoretical Studies on Copper

4.1. Introduction to Theoretical Approaches

4.1.1. Implicit Models

4.1.2. Explicit Models

4.1.3. H-shuttling Model

4.2. Theoretical Insights

4.2.1. Trends in Electrochemical Barriers

4.2.2. $\mathrm{CO}$ as a Crucial Intermediate

4.2.3. Pathway Towards Methane

4.2.4. CO Dimerization

4.2.5. $\mathrm{C}_{2}$ Pathways

4.2.6. Kinetics

4.2.7. Rate-Limiting Electron Transfers

4.3. Summary

5. Electrochemical $\mathrm{CO}_{2}$ Reduction Pathways on $\mathrm{Cu}$

6. Nanostructured Copper

6.1. Nanoparticles 
6.1.1. Nanoparticle Size Effects

6.1.2. Loading/Interparticle Distance

6.1.3. Shape Effects

6.1.4. Effect of Support and Catalyst-Ionomer Interaction

6.2. Three-Dimensionally Interconnected Catalyst Materials

6.2.1. Oxide-Derived Copper

6.2.2. Non-Oxide-Derived copper

6.2.3. The Role of Oxygen in $\mathrm{CO}_{2} \mathrm{R}$ Electrocatalysis

6.2.4. Activity Comparisons

6.3. Vapor-Fed Systems

6.4. Summary

7. Copper Bimetallics

7.1. Copper and $\mathrm{H}_{2}$ Producing Guest Metals (Fe, Ni, Pt, Pd)

7.2. Copper and CO Producing Guest Metals (Ag, $\mathrm{Au}, \mathrm{Zn}$ )

7.2.1. Synergistic Effects for CO Production

7.2.2. Synergistic Effects for $>2 \mathrm{e}^{-}$Products

7.3. Copper and $\mathrm{HCOO}^{-}$Producing Guest Metals (In, Sn)

7.4. Activity Comparisons

7.5. Summary

8. Conclusions \& Future Outlook

Acknowledgements

Supporting Information

References 


\section{Introduction}

There is a pressing need to advance the development of $\mathrm{CO}_{2}$ utilization technologies such as electrochemical $\mathrm{CO}_{2}$ reduction $\left(\mathrm{CO}_{2} \mathrm{R}\right)$; as a result, this has been a rapidly expanding field of research in recent years. In particular, there is a large body of work on copper $(\mathrm{Cu})$ materials for this reaction, since $\mathrm{Cu}$ is, as of yet, unique in its ability to catalyze the electrochemical conversion of $\mathrm{CO}_{2}$ to valuable fuels and chemicals. While many great review and perspective articles have been published in this area, they have typically had a more narrow focus either in terms of timespan or topics covered. ${ }^{1-21}$ Here we aim to provide an organized and comprehensive review of $\mathrm{CO}_{2} \mathrm{R}$ on copper and the multitude of approaches that have been used to tune its catalytic activity and selectivity. The scope strives to include all studies of electrochemical $\mathrm{CO}_{2} \mathrm{R}$ in aqueous electrolytes on $\mathrm{Cu}$-based catalysts, beginning with many of the early works in the 1980s-90s through those published in 2018, and takes into consideration both experimental and theoretical methods, planar and nanostructured $\mathrm{Cu}$, and $\mathrm{Cu}$ modified with other elements. This work is also a perspective, in which we critically analyze the literature in attempts to normalize/compare the various results and show general trends throughout the field. Additionally, we suggest several techniques and protocols that can allow for more reliable comparisons, enable a more fundamental understanding of the catalysis, and help drive this technology towards commercialization. This chapter first provides a broad context by summarizing anthropogenic carbon streams, comparing different strategies to recycle $\mathrm{CO}_{2}$, and touching upon the economics of electrochemical $\mathrm{CO}_{2}$ conversion. Then, planar polycrystalline copper is introduced as a unique electrocatalyst for this reaction, which will serve as the basis for all the discussions that follow.

\subsection{Motivation for $\mathrm{CO}_{2}$ Recycling}

At the core of biological metabolism is the ability to convert carbon between different oxidation states in order to store and release energy, as well as to synthesize functional molecules. Likewise, the oxidation of carbon is at the center of human civilization's collective "industrial metabolism" consisting of our energy infrastructure and chemical industry. Whereas in biological metabolism, reduction of $\mathrm{CO}_{2}$ 
in photosynthesis balances the oxidation of carbon in cellular respiration, carbon reduction is as of yet a missing piece of humanity's industrial metabolism. This imbalance has become a significant perturbation to Earth's natural carbon cycle, as indicated in the top two sections of Table 1. The resulting accumulation of the greenhouse gas $\mathrm{CO}_{2}$ in the atmosphere is the primary driver of today's climate change. ${ }^{22}$ While the Paris Climate Accord commits signatories to the goal of achieving "a balance between anthropogenic emissions by sources and removals by sinks of greenhouse gases in the second half of this century," ${ }^{23}$ it leaves it to individual nations to volunteer the specifics of how to achieve this goal. The problem is urgent: net $\mathrm{CO}_{2}$ emissions need to decrease rapidly and cross zero around the year 2050 if global warming is to be limited to the relatively safe level of $1.5^{\circ} \mathrm{C}$ above preindustrial levels. ${ }^{24}$ Strategies to reduce net $\mathrm{CO}_{2}$ emissions fall under three categories: decarbonization, carbon sequestration, and carbon recycling, all three of which will most likely need to play a role. This review will focus in-depth on the scientific progress and challenges of carbon recycling based on electrochemical $\mathrm{CO}_{2}$ reduction on $\mathrm{Cu}$ catalysts. For context, we will first briefly comment on decarbonization and carbon sequestration, as well as the other major $\mathrm{CO}_{2}$ recycling strategies.

\subsubsection{Closing the Carbon Cycle}

Significant progress is presently being made in decarbonization. Economic growth is increasingly decoupled from energy consumption and from $\mathrm{CO}_{2}$ emissions (Figure 1), ${ }^{25-27}$ electricity from renewable sources such as wind turbines and photovoltaics is increasing in volume and decreasing in price ${ }^{28}$ private investment in renewable energy is outpacing investment in fossil fuels, ${ }^{29}$ and global annual $\mathrm{CO}_{2}$ emissions are plateauing. ${ }^{25}$ However, significant challenges remain for full decarbonization..$^{30}$ Firstly, renewable energy sources such as wind and solar are variable and produce electrical power, which has to be utilized immediately or stored. While a number of energy storage technologies exist, including pumped hydro, compressed air, batteries, redox flow batteries, and

flywheels, they all have limitations in terms of scalability, versatility, and/or maximum storage time..$^{31,32}$ As of yet there is no technology in use that could feasibly store enough renewable electricity to power society through a cloudy and windless day. Secondly, not all energy is easily electrifiable. In transport, 
especially, which accounts for $14 \%$ of global $\mathrm{CO}_{2}$ emissions ${ }^{22}$ (middle portion of Table 1), there are clear advantages to energy in the form of liquid fuel. Finally, not all $\mathrm{CO}_{2}$ emissions are due to energy use. The bottom section of Table 1 shows a lower-bound estimation to the direct non-energy carbon use in industry, calculated as the stoichiometric amount of carbon used or released globally in the production of several key products:cement, ${ }^{33}$ steel,,${ }^{34}$ plastic,${ }^{35}$ ammonia, ${ }^{36}$ and aluminum..${ }^{37}$ Together, these products involve as much carbon as $12 \%$ of global carbon emissions. Increased recycling can help to lower this number, and decarbonization may be possible, if difficult, in some of these industries. ${ }^{30}$ However, it is clear that industry would continue to have an appetite for carbon even if energy were to be fully decarbonized.

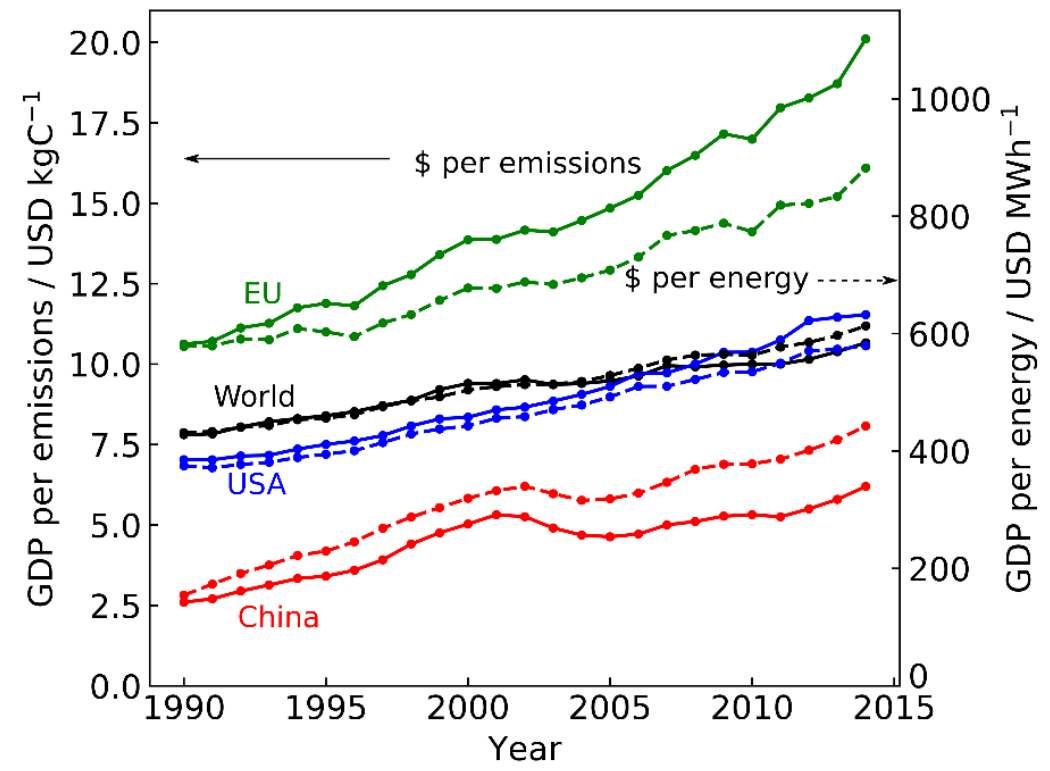

Figure 1: World GDP (purchasing power parity, 2011 dollars) is growing relative to energy consumption and $\mathrm{CO}_{2}$ emissions. Data obtained from The World Bank. ${ }^{27}$

Table 1: Global carbon fluxes

\begin{tabular}{|c|c|c|}
\hline Carbon flux & $\begin{array}{c}\text { Amount } / \\
{[\mathbf{G t C / y r}]}\end{array}$ & Year ${ }^{\text {citation }}$ \\
\hline Carbon cycle overview & 120 & $2007^{38}$ \\
\hline Air-land natural exchange & 90 & $2007^{38}$ \\
Air-ocean natural exchange & 11 & $2014^{39}$ \\
Anthropogenic carbon emissions & 13 & $2010^{22}$ \\
\hline Total anthropogenic GHG emissions by $\mathrm{CO}_{2}$ equivalent & 4 & $2014^{39}$ \\
\hline Fate of anthropogenic emissions & 3 & $2014^{39}$
\end{tabular}




\begin{tabular}{|c|c|c|}
\hline Accumulated in atmosphere & 4 & $2014^{39}$ \\
\hline \multicolumn{3}{|c|}{ Anthropogenic emissions by sector $(100 \%=13 \mathrm{GtC} / \mathrm{yr})$} \\
\hline $\begin{array}{c}\text { Electricity generation } \\
\text { Agriculture and land-use change (deforestation) } \\
\text { Industry (excluding electricity) } \\
\text { Transportation } \\
\text { Buildings } \\
\text { Other }\end{array}$ & \begin{tabular}{|c|}
$3.3(25 \%)$ \\
$3.2(24 \%)$ \\
$2.8(21 \%)$ \\
$1.9(14 \%)$ \\
$0.9(6 \%)$ \\
$1.4(10 \%)$ \\
\end{tabular} & $\begin{array}{l}2010^{22} \\
2010^{22} \\
2010^{22} \\
2010^{22} \\
2010^{22} \\
2010^{22}\end{array}$ \\
\hline \multicolumn{3}{|c|}{ Carbon in industrial reactions, $\mathrm{CO}_{2}$ as ultimate biproduct } \\
\hline $\begin{array}{c}\text { Cement (calcination): } \mathrm{CaCO}_{3} \rightarrow \mathrm{CaO}+\mathrm{CO}_{2} \\
\text { Steel (via CO): } \quad 2 \mathrm{Fe}_{2} \mathrm{O}_{3}+6 \mathrm{C}+3 \mathrm{O}_{2} \rightarrow 4 \mathrm{Fe}+6 \mathrm{CO}_{2} \\
\text { Plastic (disposal): }\left(\mathrm{CH}_{2}\right)_{2 n}+3 n \mathrm{O}_{2} \rightarrow 2 n \mathrm{H}_{2} \mathrm{O}+2 n \mathrm{CO}_{2} \\
\left.\text { Ammonia (via } \mathrm{H}_{2}\right): \quad 3 \mathrm{CH}_{4}+6 \mathrm{H}_{2} \mathrm{O}+4 \mathrm{~N}_{2} \rightarrow 8 \mathrm{NH}_{3}+3 \mathrm{CO}_{2} \\
\text { Aluminum (carbon anode): } 2 \mathrm{Al}_{2} \mathrm{O}_{3}+3 \mathrm{C} \rightarrow 4 \mathrm{Al}+3 \mathrm{CO}_{2} \\
\text { Total non-fuel carbon as industrial reactant }\end{array}$ & \begin{tabular}{|c|}
0.59 \\
0.38 \\
0.26 \\
0.045 \\
0.019 \\
1.28 \\
\end{tabular} & $\begin{array}{l}2017^{33} \\
2017^{34} \\
2015^{35} \\
2016^{36} \\
2016^{37}\end{array}$ \\
\hline \multicolumn{3}{|c|}{$\begin{array}{l}\text { Some of the carbon fluxes have been converted from carbon dioxide fluxes emissions to molecular mass }(1 \mathrm{GtC} / \mathrm{yr} \\
\left.\text { is equivalent to } 3.66 \mathrm{GtCO}_{2} / \mathrm{yr}\right) \text {. The data from Reference } 1 \text { are reported, not in terms of carbon or carbon dioxide } \\
\text { flux, but by total greenhouse gas emissions converted to the equivalent mass of } \mathrm{CO}_{2} \text { emissions based on the green } \\
\text { house gas's global warming potential. These data were converted to carbon flux as if they were } \mathrm{CO}_{2} \text { emissions. } \\
\text { The bottom section of the table shows the minimum non-energy carbon usage of the most important carbon-using } \\
\text { industrial processes, calculated based on global production and the stoichiometry of the simplified reaction } \\
\text { shown. Figures for steel and plastic exclude recycling. For plastic, we write the combustion reaction to represent } \\
\text { the stoichiometric amount of } \mathrm{CO}_{2} \text {, even though most plastic today is not incinerated but ends up in landfills or } \\
\text { the environment after use. } \\
\text { For more details, see Section } 2 \text { of the Supporting Information (SI). }\end{array}$} \\
\hline
\end{tabular}

In contrast to decarbonization, carbon sequestration is a strategy that accepts some continued net production of $\mathrm{CO}_{2}$ but aims to prevent its release to the atmosphere. ${ }^{40,41}$ There are at present 18 large-scale carbon capture and storage (CCS) projects in operation worldwide, separating about 40 megatonnes of $\mathrm{CO}_{2}$ per year $(9.6 \mathrm{MtC} / \mathrm{yr})$, from industrial point sources and storing it underground. ${ }^{42}$ This is just under $0.1 \%$ of global carbon emissions, but this could in principle be scaled up indefinitely as there are suitable geological formations for the storage of hundreds of years of $\mathrm{CO}_{2}$ at the present emissions rate. ${ }^{43}$ In particular, if the carbon stored underground originates from the atmosphere rather than from fossil fuels, as the case when $\mathrm{CO}_{2}$ resulting from the combustion of biomass is stored, the result is net-negative emissions. This strategy, bio-energy with carbon capture and storage (BECCS), can provide electricity at the same time as net-negative emissions, and will be necessary for stabilizing the climate in all but the most optimistic projections, but is as of yet largely unused. ${ }^{24}$ Unfortunately for net $\mathrm{CO}_{2}$ emissions, most existing and planned projects for CCS fall under the category of enhanced oil 
recovery (EOR), whereby $\mathrm{CO}_{2}$ is pumped underground to aid in the extraction of additional fossil fuels. ${ }^{42}$ This highlights one of the main challenges with carbon sequestration: the lack of a viable business case. $\mathrm{CO}_{2}$ separation from a power plant, for example, costs on the order of $\$ 60$ to $\$ 100 \mathrm{USD}$ per ton $\mathrm{CO}_{2}$ (approximately $\$ 200$ to $\left.\$ 350 \mathrm{USD} / \mathrm{tC}\right),{ }^{40}$ and together with transportation and storage costs, this results in a total increase of more than $25 \%$ in the cost of the electricity generated by a power plant utilizing CCS. ${ }^{44}$ Under existing policies and economics, carbon sequestration is thus rarely economically viable without EOR, which in effect adds value to the $\mathrm{CO}_{2}$ by using it to facilitate the extraction of additional fossil fuels. The immense potential of CCS, and in particular BECCS, for reducing net carbon emissions will only be realized once regulations make the cost of emitting $\mathrm{CO}_{2}$ higher than the costs of separation and storage..$^{40}$

The limitations to full implementation of decarbonization and carbon sequestration outlined above all point to a common missing piece of human civilization's collective "industrial metabolism," namely carbon recycling based on the conversion of $\mathrm{CO}_{2}$ to more reduced products. $\mathrm{CO}_{2}$ recycling can enable the storage of renewable electricity from intermittent sources in a dense and versatile form, provide a renewable carbon feedstock to chemical industries, and add value to $\mathrm{CO}_{2}$ captured at industrial point sources or directly from the air.

\subsubsection{Strategies for $\mathrm{CO}_{2}$ Recycling}

$\mathrm{CO}_{2}$ is the most oxidized form of carbon, together with carbonate minerals, with a formal oxidation state of +4 . As a result, conversion of $\mathrm{CO}_{2}$ into a more energetic product involves transfer of electrons to carbon, reducing its oxidation state. The family of reactions by which $\mathrm{CO}_{2}$ is converted to a more reduced product is most often called $\mathrm{CO}_{2}$ reduction. These reactions are also often referred to as $\mathrm{CO}_{2}$ hydrogenation for thermally-driven processes involving reaction with hydrogen $\left(\mathrm{H}_{2}\right)$ or $\mathrm{CO}_{2}$ fixation in natural photosynthesis and bio-inspired catalysis. ${ }^{45}$

In order for the production and ultimate combustion of $\mathrm{CO}_{2}$ reduction/hydrogenation products to represent a closed cycle, the ultimate source of electrons and protons for the reduction of $\mathrm{CO}_{2}$ must be water $\left(\mathrm{H}_{2} \mathrm{O}\right)$, as combustion of a hydrogenated carbon product releases $\mathrm{H}_{2} \mathrm{O} . \mathrm{CO}_{2}$ reduction / 
hydrogenation / fixation thus follows the overall formula:

$$
x \mathrm{CO}_{2}+y \mathrm{H}_{2} \mathrm{O} \rightarrow \text { product }+\mathrm{zO}_{2}
$$

Natural photosynthesis by plants and other phototrophs fixates a much larger amount of carbon globally than human activity releases (Table 1), ${ }^{38,39}$ so using biomass as a carbon source is an obvious carbon recycling strategy. However, the utility of bio-based carbon is limited by the amount of land required. Well-intended markets for transportation biofuels such as ethanol and biodiesel need energyintensive care (e.g. fertilizers, pesticides, etc.) and have led to deforestation in some cases, thus greatly mitigating or even eliminating any potential $\mathrm{CO}_{2}$ emission savings. ${ }^{46,47}$ As mentioned above, biomass energy with carbon capture and storage (BECCS), has the potential of achieving net negative emissions, however the land requirement is immense. Near-term availability of biomass for BECCS in the US, for example, corresponds at most to about $370 \mathrm{MtCO}_{2} / \mathrm{yr}(0.1 \mathrm{GtC} / \mathrm{yr})$, less than $10 \%$ of US emissions. ${ }^{48}$ The picture is similar on a global scale. ${ }^{49}$ High-yield biomass such as short-rotation forestry can yield up to about $800 \mathrm{tC} / \mathrm{km}^{2} / \mathrm{yr},{ }^{50}$ meaning that it would require about 14 million square kilometers to offset the present global carbon emissions of $11 \mathrm{GtC} / \mathrm{yr}$. Repurposing this amount of land, about the same area as is used globally for food crop production, ${ }^{22}$ is clearly not feasible. Thus, while important, harnessing the power of natural photosynthesis can only play a limited role in reaching net-zero emissions, leaving room for other $\mathrm{CO}_{2}$ recycling strategies.

There are pathways in the traditional chemical industry, all thermally activated, that could be used for $\mathrm{CO}_{2}$ hydrogenation, using $\mathrm{H}_{2}$ as the reductant. ${ }^{51,52} \mathrm{CO}_{2}$ can be reduced to carbon monoxide (CO) by the reverse water gas shift reaction:

$$
\mathrm{CO}_{2}+\mathrm{H}_{2} \rightarrow \mathrm{CO}+\mathrm{H}_{2} \mathrm{O}
$$

(2) Reverse water - gas shift

If an excess of $\mathrm{H}_{2}$ is used and the water is condensed out, the product gas is a mixture of $\mathrm{H}_{2}$ and $\mathrm{CO}$, which is called synthesis gas or syngas. Syngas can be used as the precursor to methane on a $\mathrm{Ni}$ catalyst,,$^{53}$ to multi-carbon hydrocarbons on an $\mathrm{Fe}$ or Co catalyst, ${ }^{54}$ or to methanol on a $\mathrm{Cu} / \mathrm{ZnO}$ catalyst. ${ }^{55,56}$ These reactions are called methanation, the Fischer-Tropsch reaction, and methanol synthesis, respectively.

$$
\begin{array}{ll}
\mathrm{CO}+3 \mathrm{H}_{2} \rightarrow \mathrm{CH}_{4}+\mathrm{H}_{2} \mathrm{O} & \text { (3) Methanation } \\
n \mathrm{CO}+(2 n+1) \mathrm{H}_{2} \rightarrow \mathrm{C}_{n} \mathrm{H}_{2 n+2}+n_{2} \mathrm{O}
\end{array}
$$


In methanol synthesis, some $\mathrm{CO}_{2}$ is required in the syngas stream because $\mathrm{CO}_{2}$ is actually the immediate reactant. ${ }^{57}$ The role of $\mathrm{CO}$ is to react with the water released by $\mathrm{CO}_{2}$ hydrogenation and generate more $\mathrm{CO}_{2}$ via the water-gas shift (reverse of Reaction 2). The net reaction is:

$$
\mathrm{CO}+2 \mathrm{H}_{2} \rightarrow \mathrm{CH}_{3} \mathrm{OH} \quad \text { (5) Water - gas shift plus methanol synthesis }
$$

All of these processes run at high pressures and temperatures; for example, $\sim 100$ bar and $250{ }^{\circ} \mathrm{C}$ are typical conditions for methanol synthesis, the mildest of the three reactions. ${ }^{52}$

In order for $\mathrm{CO}_{2}$ hydrogenation by the above reactions to be a renewable process, the hydrogen must come from water splitting using renewable energy:

$$
2 \mathrm{H}_{2} \mathrm{O} \rightarrow \mathrm{O}_{2}+2 \mathrm{H}_{2} \quad \text { (6) Water splitting }
$$

such that the combined reaction is on the form of Reaction 1 above. The water splitting would be driven electrochemically using electrical energy from renewable sources such as wind and solar, ${ }^{30,58,59}$ according to the half-reactions:

$$
\begin{array}{ll}
2 \mathrm{H}^{+}+2 e^{-} \rightarrow \mathrm{H}_{2} & \text { (7) } \mathrm{H}_{2} \text { Evolution Reaction (HER) at the cathode } \\
2 \mathrm{H}_{2} \mathrm{O} \rightarrow \mathrm{O}_{2}+4 \mathrm{H}^{+}+4 e^{-} & \text {(8) } \mathrm{O}_{2} \text { Evolution Reaction (OER) at the anode }
\end{array}
$$

Compared to hydrogen, carbon-based fuels have the advantages of higher volumetric energy density and greater ease of integration in the present infrastructure (drop-in fuels). Furthermore, carbonbased chemicals will still be needed regardless of energy decarbonization. ${ }^{60}$

An alternative to $\mathrm{CO}$ production by the reverse water-gas shift (Reaction 2) is electrochemical reduction of $\mathrm{CO}_{2}$ to $\mathrm{CO}$ :

$$
\mathrm{CO}_{2}+2 \mathrm{H}^{+}+2 e^{-} \rightarrow \mathrm{CO}+\mathrm{H}_{2} \mathrm{O}
$$

\section{Electrochemical CO production}

balanced by the OER (reaction 8). The electrochemically produced $\mathrm{CO}$ can then be reacted further as described above to form hydrocarbons or alcohols from $\mathrm{CO}_{2}$. This combined approach using electroreduction of $\mathrm{CO}_{2}$ in a high-temperature solid-oxide electrolysis cell and subsequent thermal hydrogenation proved especially promising in an analysis of $\mathrm{CO}_{2}$ recycling strategies. ${ }^{61}$

However, direct electrochemical reduction of $\mathrm{CO}_{2}$ can have a number of advantages compared to hydrogenation of $\mathrm{CO}_{2}$ or $\mathrm{CO}$ by electrochemically produced $\mathrm{H}_{2} \cdot{ }^{2,62}$ it (1) combines the electrochemical water splitting and subsequent thermal hydrogenation into a single electrochemical 
process, (2) enables products that cannot easily be prepared by thermally driven processes, and (3) can often run at or near room temperature and ambient pressure. These features imply that processes based on electrochemical $\mathrm{CO}_{2}$ reduction are more suitable to decentralization than the thermal counterparts, and that electrochemical $\mathrm{CO}_{2}$ reduction processes can be designed to enable relatively quick adjustment of production in order to match the overproduction of electricity from intermittent renewable sources. ${ }^{63}$

\subsection{Electrochemical $\mathrm{CO}_{2}$ Reduction}

In electrochemical $\mathrm{CO}_{2}$ reduction, the cathodic reaction is of the general form:

$$
x \mathrm{CO}_{2}+n \mathrm{H}^{+}+n e^{-} \rightarrow \text { product }+y \mathrm{H}_{2} \mathrm{O} \quad \text { (9) } \mathrm{CO}_{2} \text { reduction }\left(\mathrm{CO}_{2} \mathrm{R}\right)
$$

Like in water splitting, the anodic reaction in electrochemical $\mathrm{CO}_{2} \mathrm{R}$ must be the oxygen evolution reaction (OER, Reaction 8) in order to sum to the overall reaction given by Reaction 1 above. In other words, water is the only renewable and scalable source of electrons and protons. ${ }^{64}$ In this section, we first discuss the carbon dioxide reduction reaction in general, with a focus on the thermodynamics and economics of reducing $\mathrm{CO}_{2}$ to various products. We then summarize the actual $\mathrm{CO}_{2} \mathrm{R}$ activity and selectivity of metal electrodes, highlighting the unique ability of copper to reduce $\mathrm{CO}_{2}$ to hydrocarbons and alcohols.

\subsubsection{Desired Products: Thermodynamics and Economics}

Table 2 lists the equilibrium potentials for $\mathrm{CO}_{2} \mathrm{R}$ to commonly reported electrochemical products. All of the $\mathrm{CO}_{2} \mathrm{R}$ standard potentials here are calculated via the Gibbs free energy of reaction using gas-phase thermochemistry data and, for aqueous products, Henry's Law data, from NIST. ${ }^{65} \mathrm{CO}_{2}$ is always considered a gas, water a liquid, and the state of the product is gas or aqueous as indicated. Regardless of which $\mathrm{CO}_{2} \mathrm{R}$ product is formed at the cathode, the fact that the $\mathrm{CO}_{2} \mathrm{R}$ reaction together with OER (Reaction 8) must add to an overall reaction of the form of Reaction 1 means that the stoichiometric coefficients for electrons and protons in a sustained $\mathrm{CO}_{2}$ reduction reaction must be equal. Acidic products are therefore considered in the fully protonated form. Similar tables and lists provided 
in previous $\mathrm{CO}_{2} \mathrm{R}$ reviews, ${ }^{1,2,15,16,45}$ studies of $\mathrm{CO}_{2} \mathrm{R}$ electrocatalysis, ${ }^{66}$ and process/economic analyses for electrochemical $\mathrm{CO}_{2}$ conversion ${ }^{67-70}$ all show some variation due to the use of different potential scales, deprotonated products, and/or different standard states (for instance liquid instead of aqueous).

As described in detail in subsequent sections of this review, $\mathrm{CO}$ is an important intermediate in $\mathrm{CO}_{2} \mathrm{R}$ to hydrocarbons, aldehydes, and alcohols on $\mathrm{Cu}$, and a promising approach to $\mathrm{CO}_{2} \mathrm{R}$ is a stepwise one via $\mathrm{CO}$, in which $\mathrm{CO}$ reduction $(\mathrm{COR})$ is an important reaction in its own right. ${ }^{71}$ The equilibrium potential for $\mathrm{CO}$ reduction to a few products is therefore also included in this table, and an equation relating $\mathrm{CO}$ reduction equilibrium potential to $\mathrm{CO}_{2}$ reduction equilibrium potential is given in Section 1 of the Supporting Information (SI).

Table 2: Electrochemical reactions with equilibrium potentials

\begin{tabular}{|c|c|c|}
\hline Reaction & $\begin{array}{c}\mathbf{E}^{\mathbf{0}} / \\
{[\mathrm{V} \text { vs. } \mathrm{RHE}]}\end{array}$ & (Product) Name, abbreviation \\
\hline $\begin{array}{l}2 \mathrm{H}_{2} \mathrm{O} \rightarrow \mathrm{O}_{2}+4 \mathrm{H}^{+}+4 \mathrm{e}^{-} \\
2 \mathrm{H}^{+}+2 \mathrm{e}^{-} \rightarrow \mathrm{H}_{2}\end{array}$ & $\begin{array}{c}1.23 \\
0\end{array}$ & $\begin{array}{l}\text { Oxygen Evolution Reaction, OER } \\
\text { Hydrogen Evolution Reaction, HER }\end{array}$ \\
\hline$x \mathrm{CO}_{2}+n \mathrm{H}^{+}+n e^{-} \rightarrow$ Product $+y \mathrm{H}_{2} \mathrm{O}$ & & $\mathrm{CO}_{2}$ Reduction, $\mathrm{CO}_{2} \mathrm{R}$ \\
\hline $\mathrm{CO}_{2}+2 \mathrm{H}^{+}+2 \mathrm{e}^{-} \rightarrow \mathrm{HCOOH}_{(\mathrm{aq})}$ & -0.12 & Formic acid \\
\hline $\mathrm{CO}_{2}+2 \mathrm{H}^{+}+2 \mathrm{e}^{-} \rightarrow \mathrm{CO}_{(\mathrm{g})}+\mathrm{H}_{2} \mathrm{O}$ & -0.10 & Carbon monoxide \\
\hline $\mathrm{CO}_{2}+6 \mathrm{H}^{+}+6 \mathrm{e}^{-} \rightarrow \mathrm{CH}_{3} \mathrm{OH}_{(\mathrm{aq})}+\mathrm{H}_{2} \mathrm{O}$ & 0.03 & Methanol, MeOH \\
\hline $\mathrm{CO}_{2}+4 \mathrm{H}^{+}+4 \mathrm{e}^{-} \rightarrow \mathrm{C}_{(\mathrm{s})}+2 \mathrm{H}_{2} \mathrm{O}$ & 0.21 & Graphite \\
\hline $\mathrm{CO}_{2}+8 \mathrm{H}^{+}+8 \mathrm{e}^{-} \rightarrow \mathrm{CH}_{4}(\mathrm{~g})+2 \mathrm{H}_{2} \mathrm{O}$ & 0.17 & Methane \\
\hline $2 \mathrm{CO}_{2}+2 \mathrm{H}^{+}+2 \mathrm{e}^{-} \rightarrow(\mathrm{COOH})_{2(\mathrm{~s})}$ & -0.47 & Oxalic acid \\
\hline $2 \mathrm{CO}_{2}+8 \mathrm{H}^{+}+8 \mathrm{e}^{-} \rightarrow \mathrm{CH}_{3} \mathrm{COOH}_{(\mathrm{aq})}+2 \mathrm{H}_{2} \mathrm{O}$ & 0.11 & Acetic acid \\
\hline $2 \mathrm{CO}_{2}+10 \mathrm{H}^{+}+10 \mathrm{e}^{-} \rightarrow \mathrm{CH}_{3} \mathrm{CHO}_{(\mathrm{aq})}+3 \mathrm{H}_{2} \mathrm{O}$ & 0.06 & Acetaldehyde \\
\hline $2 \mathrm{CO}_{2}+12 \mathrm{H}^{+}+12 \mathrm{e}^{-} \rightarrow \mathrm{C}_{2} \mathrm{H}_{5} \mathrm{OH}_{(\mathrm{aq})}+3 \mathrm{H}_{2} \mathrm{O}$ & 0.09 & Ethanol, EtOH \\
\hline $2 \mathrm{CO}_{2}+12 \mathrm{H}^{+}+12 \mathrm{e}^{-} \rightarrow \mathrm{C}_{2} \mathrm{H}_{4(\mathrm{~g})}+4 \mathrm{H}_{2} \mathrm{O}$ & 0.08 & Ethylene \\
\hline $2 \mathrm{CO}_{2}+14 \mathrm{H}^{+}+14 \mathrm{e}^{-} \rightarrow \mathrm{C}_{2} \mathrm{H}_{6(\mathrm{~g})}+4 \mathrm{H}_{2} \mathrm{O}$ & 0.14 & Ethane \\
\hline $3 \mathrm{CO}_{2}+16 \mathrm{H}^{+}+16 \mathrm{e}^{-} \rightarrow \mathrm{C}_{2} \mathrm{H}_{5} \mathrm{CHO}_{(\mathrm{aq})}+5 \mathrm{H}_{2} \mathrm{O}$ & 0.09 & Propionaldehyde \\
\hline $3 \mathrm{CO}_{2}+18 \mathrm{H}^{+}+18 \mathrm{e}^{-} \rightarrow \mathrm{C}_{3} \mathrm{H}_{7} \mathrm{OH}_{(\mathrm{aq})}+5 \mathrm{H}_{2} \mathrm{O}$ & 0.10 & Propanol, $\mathrm{PrOH}$ \\
\hline$x \mathrm{CO}+n \mathrm{H}^{+}+n e^{-} \rightarrow$ Product $+y \mathrm{H}_{2} \mathrm{O}$ & & CO Reduction, COR \\
\hline $\mathrm{CO}+6 \mathrm{H}^{+}+6 \mathrm{e}^{-} \rightarrow \mathrm{CH}_{4(\mathrm{~g})}+\mathrm{H}_{2} \mathrm{O}$ & 0.26 & Methane \\
\hline $2 \mathrm{CO}+8 \mathrm{H}^{+}+8 \mathrm{e}^{-} \rightarrow \mathrm{CH}_{3} \mathrm{CH}_{2} \mathrm{OH}_{(\mathrm{aq})}+\mathrm{H}_{2} \mathrm{O}$ & 0.19 & Ethanol, EtOH \\
\hline $2 \mathrm{CO}+8 \mathrm{H}^{+}+8 \mathrm{e}^{-} \rightarrow \mathrm{C}_{2} \mathrm{H}_{4(\mathrm{~g})}+2 \mathrm{H}_{2} \mathrm{O}$ & 0.17 & Ethylene \\
\hline
\end{tabular}

We recommend reporting and using potentials on the thermodynamically relevant and $\mathrm{pH}$ independent RHE scale for all calculations of overpotential and energetic efficiency. For more discussion of thermodynamics and equilibrium potentials, please see Section 1 of the SI. 
While the equilibrium potential of $\mathrm{CO}_{2}$ reduction to hydrocarbons, aldehydes, and alcohols is slightly positive of RHE, their direct electrochemical production still requires significant energy input, as the minimum possible potential to drive the overall reaction (i.e., the difference between the $\mathrm{CO}_{2} \mathrm{R}$ equilibrium potential and the equilibrium potential of OER) is larger than $1 \mathrm{~V}$. Furthermore, both the OER and $\mathrm{CO}_{2} \mathrm{R}$ currently require large overpotentials, meaning that a $\mathrm{CO}_{2} \mathrm{R}$ device will run at a larger cell potential than this thermodynamic potential. Nonetheless, the thermodynamic cell potential indicates the minimum energy requirement for a given product, and thus, given a cost of energy, provides a means to estimate the economic viability of a product. More thorough techno-economic analyses have also been performed, which include capital and operational costs and assumptions about the overpotential and Faradaic efficiency of the desired $\mathrm{CO}_{2}$ reduction reaction. ${ }^{67,69,70,72}$

Figure 2 shows a simple mapping of the economics and energetics of possible $\mathrm{CO}_{2}$ reduction products. The approximate market price of selected possible $\mathrm{CO}_{2}$ reduction products are plotted against the minimum energy needed for their production by $\mathrm{CO}_{2} \mathrm{R}$ balanced with OER. The marker size indicates the (logarithmic) size of the global market, which spans more than four orders of magnitude. All economic quantities are normalized to mass of carbon. The dashed and dotted lines indicate the minimum cost of production given a captured $\mathrm{CO}_{2}$ price of $\$ 200 / \mathrm{tC}$ and an electricity price of $\$ 50 / \mathrm{MWh}$, or $\$ 20 / \mathrm{MWh}$, respectively. $\$ 200 / \mathrm{tC}$ is a representative price for carbon captured from a power plant. ${ }^{40}$ $\$ 50 / \mathrm{MWh}$ is representative of recent solar installations ${ }^{29}$ Current record lows for solar installation are around \$20/MWh, which will likely become more common in the future. Products above the line for a given $\mathrm{CO}_{2}$ capture cost and electricity cost may be economically feasible depending on capital and other costs, which are not considered here, but are in other analyses. Products below the line are not economically feasible. Direct air capture is estimated to cost $\$ 2000 / \mathrm{tC}$ with current technologies, ${ }^{40}$ which would push the lines up above nearly all products, though this will likely come down in the future. Taxes on carbon emissions push the effective price of captured $\mathrm{CO}_{2}$ down, since capturing $\mathrm{CO}_{2}$ avoids the tax. The raw data, sources, and calculations are given in Section 2 of the SI. For a more full picture we refer the reader to techno-economic analyses of $\mathrm{CO}_{2}$ reduction in the literature..$^{67,69,70,72}$ 


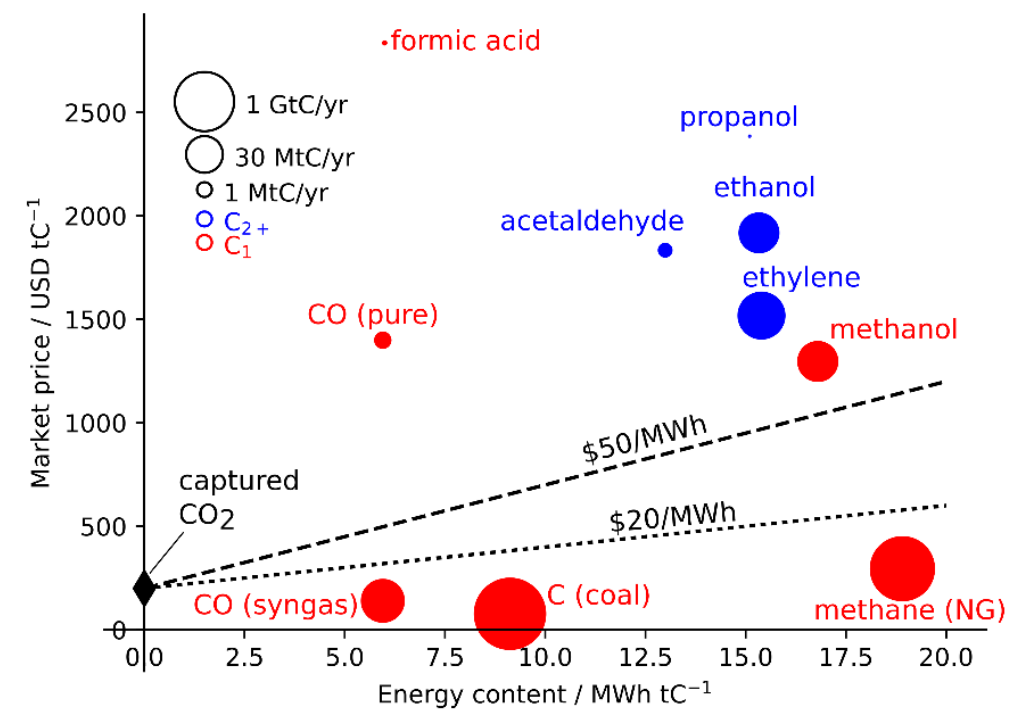

Figure 2: Market price of select $\mathrm{CO}_{2}$ recycling products as a function of energy content. Lines represent minimum energy and $\mathrm{CO}_{2}$ costs. Capital costs are not considered.

Despite its simplicity, Figure 2 captures a widely shared conclusion: ${ }^{28,40,69,70,73,74} \mathrm{CO}_{2}$ reduction

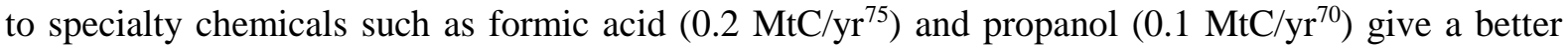
chance of near-term economic feasibility, whereas making an impact on the GtC/year scale relevant for global climate change will likely require renewable production of fuels competitive with the likes of coal $\left(4 \mathrm{GtC} / \mathrm{yr}^{22}\right)$ and natural gas $\left(1.4 \mathrm{GtC} / \mathrm{yr}^{76}\right)$. This is not feasible in the near term, as coal and natural gas are cheaper than the minimum energy required to synthesize them from $\mathrm{CO}_{2}$ even at $\$ 20 / \mathrm{MWh}$.

Ethylene $\left(120 \mathrm{MtC} / \mathrm{yr}^{77}\right)$ and ethanol $\left(40 \mathrm{MtC} / \mathrm{yr}^{70}\right)$ represent promising compromises between profitable niche market and impactful mass market for $\mathrm{CO}_{2}$ reduction. Ethylene is produced at large scale as a precursor for plastics and ethylene glycol in a highly energy-demanding and unselective process involving repeated cycles of steam cracking at $750-950{ }^{\circ} \mathrm{C}$ followed by quenching, distillation, and recompression..$^{78,79}$ At an electricity price of $\$ 50 \mathrm{USD} / \mathrm{MWh}$, it is worth about twice as much as the minimum energy input, with a global market about 100 times larger than the specialty chemicals. Ethanol is a fuel with high volumetric energy density that can be substituted for fossil fuels in the transport industry with minimal modifications, and thus represents a market with high growth potential ${ }^{80}$ Ethanol is produced primarily as a bio-fuel today, and is sold for a price much higher than 
the minimum energy required for its production by $\mathrm{CO}_{2} \mathrm{R}$ at $\$ 50 / \mathrm{MWh}$. Ethylene and ethanol are both major products in $\mathrm{CO}_{2}$ and $\mathrm{CO}$ electroreduction on copper, as described below.

\subsubsection{Copper as a Unique Electrocatalyst}

Studies of electrochemical $\mathrm{CO}_{2}$ reduction date back to at least the 1950 's ${ }^{81}$ The first study to quantify both gaseous and liquid products, and thus account for $100 \%$ of Faradaic efficiency, was reported by Yoshio Hori and coworkers in $1985 .{ }^{82}$ In this study, constant-current electrolysis of $\mathrm{CO}_{2-}$ saturated $0.5 \mathrm{M} \mathrm{KHCO}_{3}$ at $5 \mathrm{~mA} \mathrm{~cm}{ }^{-2}$ (geometric) was performed for up to an hour as a batch experiment on a number of polycrystalline metal electrodes. This and subsequent studies utilizing basically the same methods ${ }^{83-86}$ led to a classification of metal electrodes into four groups: ${ }^{1} \mathrm{~Pb}, \mathrm{Hg}, \mathrm{Tl}, \mathrm{In}, \mathrm{Sn}, \mathrm{Cd}$, and $\mathrm{Bi}$ produce primarily formate $\left(\mathrm{HCOO}^{-}\right) ; \mathrm{Au}, \mathrm{Ag}, \mathrm{Zn}, \mathrm{Pd}$, and $\mathrm{Ga}$ produce primarily carbon monoxide $(\mathrm{CO}) ; \mathrm{Ni}, \mathrm{Fe}, \mathrm{Pt}$, and Ti reduce very little $\mathrm{CO}_{2}$ and instead almost exclusively reduce water to $\mathrm{H}_{2}$; and $\mathrm{Cu}$ stands out in uniquely producing a number of hydrocarbons, aldehydes, and alcohols. $\mathrm{Cu}$ is thus the only pure metal that reduces $\mathrm{CO}_{2}$ to products requiring more than two electrons transfers (collectively referred to as "further reduced products" or " $>2 \mathrm{e}^{-}$products") with substantial Faradaic efficiencies. These results are summarized in Table 3, adapted from Hori's 2008 review of $\mathrm{CO}_{2}$ reduction on metal electrodes. ${ }^{1}$

Table 3: Faradaic efficiencies of $\mathrm{CO}_{2}$ reduction products on metal electrodes in $\mathrm{CO}_{2}$-saturated $0.1 \mathrm{M}$ $\mathrm{K}_{2} \mathrm{CO}_{3}(\mathrm{pH}=6.8)$. The potential has been converted from the reported SHE potential to the RHE scale by adding RT/Fln $(10) \mathrm{pH}=0.40 \mathrm{~V}$. Adapted with permission from Hori. ${ }^{1}$ Copyright $\odot 2$ 2008, Springer Science Business Media, LLC.

\begin{tabular}{|c|c|c|c|c|c|c|c|c|c|c|}
\hline Metal & $\begin{array}{c}\boldsymbol{E}[\mathbf{V} \text { vs. } \\
\mathbf{R H E}]\end{array}$ & $\begin{array}{c}\boldsymbol{J}_{\text {geo }} \\
{\left[\mathbf{m A} / \mathbf{c m}^{2}\right]}\end{array}$ & $\begin{array}{c}\mathbf{C H}_{\mathbf{4}} \\
{[\%]}\end{array}$ & $\begin{array}{c}\mathbf{C}_{2} \mathbf{H}_{\mathbf{4}} \\
{[\mathbf{\%}]}\end{array}$ & $\begin{array}{c}\text { EtOH } \\
{[\%]}\end{array}$ & $\begin{array}{c}\text { PrOH } \\
{[\%]}\end{array}$ & $\begin{array}{c}\mathbf{C O} \\
{[\%]}\end{array}$ & $\begin{array}{c}\mathbf{H C O O}^{-} \\
{[\%]}\end{array}$ & $\begin{array}{c}\mathbf{H}_{\mathbf{2}} \\
{[\%]}\end{array}$ & $\begin{array}{c}\text { Total } \\
{[\%]}\end{array}$ \\
\hline $\mathrm{Pb}$ & -1.23 & -5.0 & 0 & 0 & 0 & 0 & 0 & 97.4 & 5.0 & 102.4 \\
$\mathrm{Hg}$ & -1.11 & -0.5 & 0 & 0 & 0 & 0 & 0 & 99.5 & 0 & 99.5 \\
$\mathrm{Tl}$ & -1.20 & -5.0 & 0 & 0 & 0 & 0 & 0 & 95.1 & 6.2 & 101.3 \\
$\mathrm{In}$ & -1.15 & -5.0 & 0 & 0 & 0 & 0 & 2.1 & 94.9 & 3.3 & 100.3 \\
$\mathrm{Sn}$ & -1.08 & -5.0 & 0 & 0 & 0 & 0 & 7.1 & 88.4 & 4.6 & 100.1 \\
$\mathrm{Cd}$ & -1.23 & -5.0 & 1.3 & 0 & 0 & 0 & 13.9 & 78.4 & 9.4 & 103.0 \\
\hline $\mathrm{Au}$ & -0.64 & -5.0 & 0 & 0 & 0 & 0 & 87.1 & 0.7 & 10.2 & 98.0 \\
$\mathrm{Ag}$ & -0.97 & -5.0 & 0 & 0 & 0 & 0 & 81.5 & 0.6 & 12.4 & 94.6 \\
$\mathrm{Zn}$ & -1.14 & -5.0 & 0 & 0 & 0 & 0 & 79.4 & 6.1 & 9.9 & 95.4 \\
$\mathrm{Pd}$ & -0.8 & -5.0 & 2.9 & 0 & 0 & 0 & 28.3 & 2.8 & 26.2 & 60.2 \\
$\mathrm{Ga}$ & -0.84 & -5.0 & 0 & 0 & 0 & 0 & 23.2 & 0 & 79.0 & 102.0 \\
\hline
\end{tabular}




\begin{tabular}{|c|c|c|c|c|c|c|c|c|c|c|}
\hline $\mathrm{Cu}$ & -1.04 & -5.0 & 33.3 & 25.5 & 5.7 & 3.0 & 1.3 & 9.4 & 20.5 & 103.5 \\
\hline $\mathrm{Ni}$ & -1.08 & -5.0 & 1.8 & 0.1 & 0 & 0 & 0 & 1.4 & 88.9 & 92.4 \\
$\mathrm{Fe}$ & -0.51 & -5.0 & 0 & 0 & 0 & 0 & 0 & 0 & 94.8 & 94.8 \\
$\mathrm{Pt}$ & -0.67 & -5.0 & 0 & 0 & 0 & 0 & 0 & 0.1 & 95.7 & 95.8 \\
$\mathrm{Ti}$ & -1.20 & -5.0 & 0 & 0 & 0 & 0 & tr. & 0 & 99.7 & 99.7 \\
\hline
\end{tabular}

Note that in all cases, a significant overpotential is required to reach the specified current density, usually $-5 \mathrm{~mA} / \mathrm{cm}^{2}$. The largest overpotentials are required by the formate-producing metals, which also typically show very high $\mathrm{CO}_{2} \mathrm{R}$ Faradaic efficiencies (for example, $97 \%$ of $-5 \mathrm{~mA} / \mathrm{cm}^{2}$ for $\mathrm{Pb}$ at $-1.23 \mathrm{~V}$ vs. RHE, an overpotential of $1.1 \mathrm{~V}$ ) due to very low HER activity. The smallest overpotential for $\mathrm{CO}_{2} \mathrm{R}$ is achieved by $\mathrm{Au}$, which produces $\mathrm{CO}$ with high Faradaic efficiency, $87 \%$ of $5 \mathrm{~mA} / \mathrm{cm}^{2}$ at $-0.7 \mathrm{~V}$ vs. RHE, an overpotential of $0.6 \mathrm{~V}$. Copper uniquely produces a variety of hydrocarbon and oxygenated products at an intermediate potential $-1.04 \mathrm{~V} v s$. RHE (e.g., an overpotential of $0.9 \mathrm{~V}$ for $\mathrm{CO}_{2} \mathrm{R}$ to $\mathrm{CO}$ and an overpotential of $1.2 \mathrm{~V}$ for $\mathrm{CO}_{2} \mathrm{R}$ to methane). Note also that these results, by measuring $\mathrm{CO}_{2}$ reduction selectivity for each electrode at only one current density, effectively provide only a snapshot, as $\mathrm{CO}_{2}$ reduction selectivity can change with conditions and electrode potential.

The splitting of the metals into these four groups based on their selectivity has been explained as the result of their binding energy to key $\mathrm{CO}_{2} \mathrm{R}$ and $\mathrm{HER}$ intermediates, including ${ }^{*} \mathrm{H},{ }^{*} \mathrm{OCHO}$ (bound to the surface through $\mathrm{O}$ ), ${ }^{*} \mathrm{COOH}$ (bound to the surface through $\mathrm{C}$ ), and ${ }^{*} \mathrm{CO} \cdot{ }^{87}$ The unique ability of $\mathrm{Cu}$ to reduce $\mathrm{CO}_{2}$ to $>2 \mathrm{e}^{-}$products may be due to the fact that it is the only metal that has a negative adsorption energy for $* \mathrm{CO}$ but a positive adsorption energy for $* \mathrm{H}$, as shown in Figure 3. 


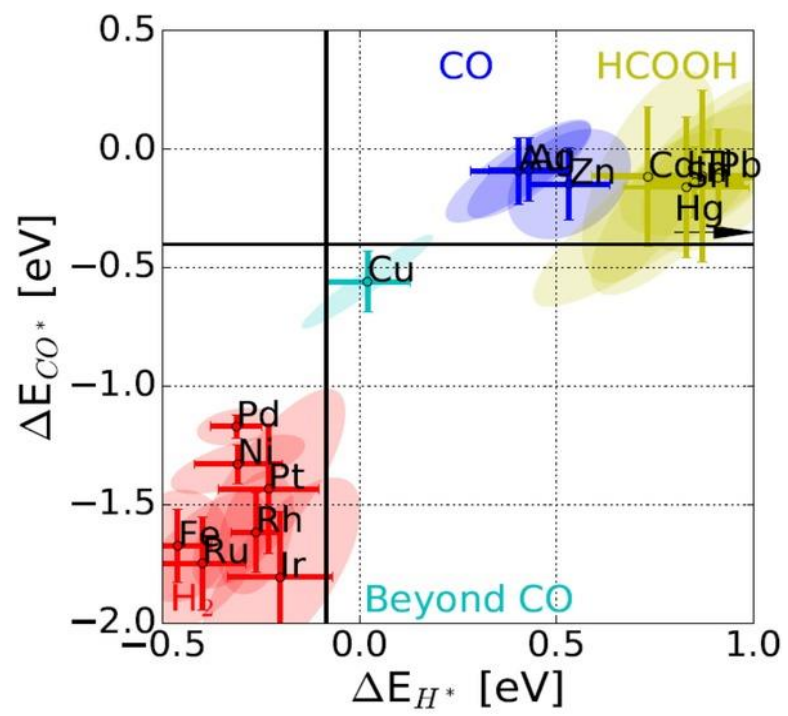

Figure 3: $\mathrm{CO}_{2}$ reduction metal classification. Reproduced with permission from Bagger et al. ${ }^{87}$ Copyright (c) 2017, John Wiley and Sons.

After the above-mentioned constant-current electrolysis experiments to compare different metal electrodes, Hori and coworkers also performed constant-potential electrolysis experiments on $\mathrm{Cu}$ in $\mathrm{CO}_{2}$-saturated $0.1 \mathrm{M} \mathrm{KHCO}_{3}(\mathrm{pH}=6.8)$, the most common electrolyte for $\mathrm{CO}_{2}$ reduction studies, to characterize the $\mathrm{CO}_{2} \mathrm{R}$ product distribution as a function of potential. ${ }^{86}$ The results, shown in Figure 4 (hollow markers) span a potential range of -0.8 to $-1.45 \mathrm{~V} v s$. NHE, or approximately -0.4 to $-1.05 \mathrm{~V}$ vs. RHE. In order of least cathodic to most cathodic onset potential (which loosely refers to the potential closest to the equilibrium potential at which a product is produced in detectable quantities), the major $\mathrm{CO}_{2}$ reduction products are $\mathrm{CO}, \mathrm{HCOO}^{-}, \mathrm{C}_{2} \mathrm{H}_{4}$, and $\mathrm{CH}_{4} . \mathrm{CO}$ and $\mathrm{HCOO}^{-}$pass through potentials of maximum Faradaic efficiency, and the Faradaic efficiency towards $\mathrm{C}_{2} \mathrm{H}_{4}$ reaches a plateau by about $1.0 \mathrm{~V}$ vs. RHE. Similar conclusions were reached years later by Kuhl et al.; ${ }^{66}$ electrolysis experiments were performed at potentials between -0.6 and $-1.2 \mathrm{~V} v s$. RHE and, using more sensitive product detection techniques for liquid products, a total of 16 products were observed. These results are coplotted with Hori's in Figure 4 (filled markers). 

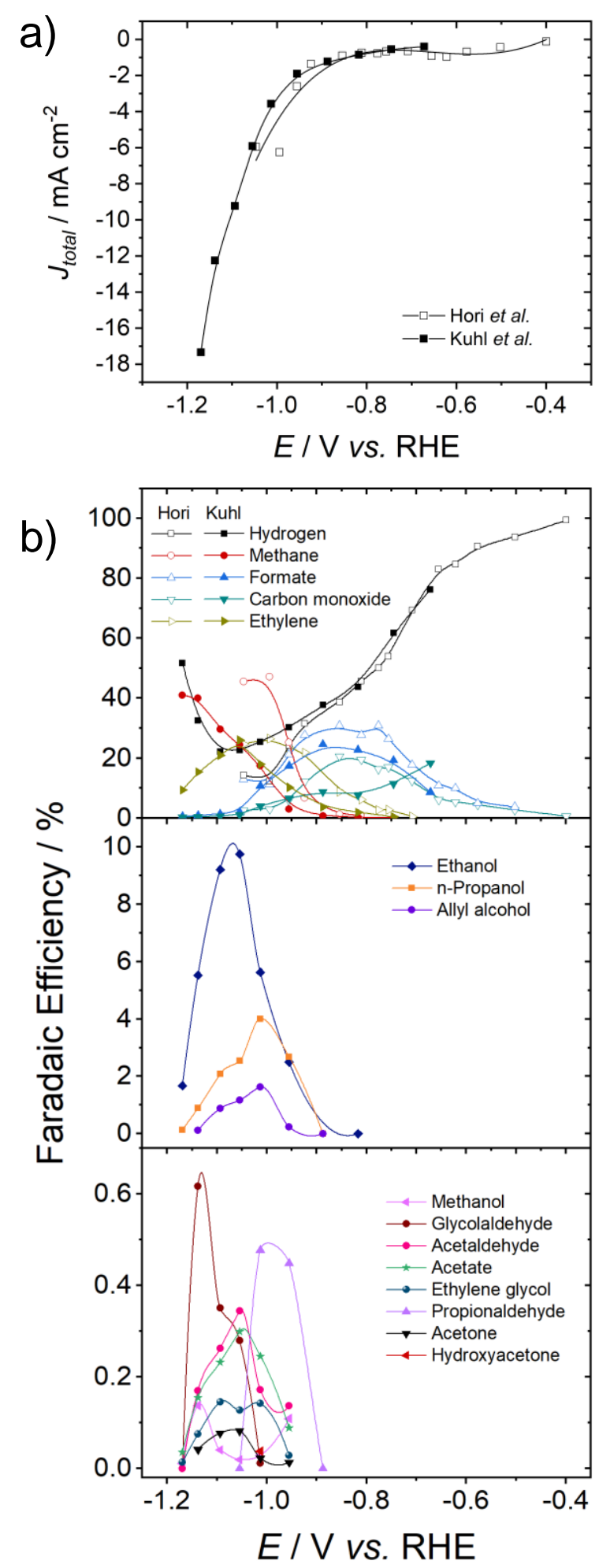

Figure 4: $\mathrm{CO}_{2} \mathrm{R}$ activity and selectivity on polycrystalline copper in $\mathrm{CO}_{2}$-saturated $0.1 \mathrm{M} \mathrm{KHCO}$ $(\mathrm{pH}=6.8)$. a) total current density and b) product Faradaic efficiencies, data obtained from Hori et al. ${ }^{86}$ (hollow) and Kuhl et al. ${ }^{66}$ (filled). 
The ability to reduce $\mathrm{CO}_{2}$ to valuable hydrocarbons, aldehydes, and alcohols is of great interest; as a result, there has been much work done in the field to better understand the reactivity of $\mathrm{Cu}$ and how it can be tuned to achieve greater selectivity, stability, and efficiency. Thus, the remainder of this work will focus exclusively on $\mathrm{Cu}$ and $\mathrm{Cu}$-based electrocatalysts. In the sections that follow, we will discuss experimental probes used to identify $\mathrm{CO}_{2} \mathrm{R}$ reaction mechanisms on $\mathrm{Cu}$ and the complementary theoretical methods \& descriptions of $\mathrm{CO}_{2} \mathrm{R}$. We will then discuss two materials classes that have been studied in attempts to alter the reactivity of planar polycrystalline $\mathrm{Cu}$ (nanostructured $\mathrm{Cu}$ and bimetallics with $\mathrm{Cu}$ ). Finally, we will provide some overall perspectives and an outlook for the future of $\mathrm{CO}_{2} \mathrm{R}$.

\section{Considerations for Conducting \& Comparing Electrochemical $\mathrm{CO}_{2}$ Reduction Experiments}

Before diving into the results in subsequent sections, we would like to set the stage by discussing some of the difficulties encountered when trying to reliably compare the electrocatalytic behavior of $\mathrm{Cu}$ between different electrochemical cells and conditions. For more detailed discussions of these challenges in an objective evaluation of $\mathrm{CO}_{2} \mathrm{R}$ performance, we direct the reader to recent perspective articles, which also provide recommendations to help standardize methods for measuring and reporting $\mathrm{CO}_{2} \mathrm{R}$ data. ${ }^{88,89}$

\subsection{Reporting Data}

First we would briefly like to discuss the metrics by which electrocatalytic activity and selectivity are reported. The selectivity is most often described in terms of Faradaic efficiency, which is the portion of the electrical current going to a specific product in steady-state electrolysis. The activity for $\mathrm{CO}_{2} \mathrm{R}$ to a specific product is described in terms of the overpotential, which is the absolute difference between the actual potential needed to make a product and the thermodynamic potential, or in terms of the partial current density at a given potential, which is the total current times the Faradaic efficiency. A more active catalyst is one that achieves a given partial current density at a lower overpotential, and/or 
provides a larger partial current density at a given overpotential. A major goal of applied electrocatalysis research is the development of electrode materials and conditions that are selective and active (as well as cheap and stable) for production of desired products. However, understanding the fundamentals of electrocatalysis on simple materials and model systems facilitates the design of better electrodes ${ }^{58}$ and the tradeoff between practical application and fundamental insight is important to keep in mind when comparing different works in the field.

An essential consideration is how the measured activity is normalized. The geometric area of the electrode is most commonly used to report the current density. While useful for assessing the overall electrode performance, this metric is inherently dependent on the catalyst loading or surface area. Thus, it is also important to consider the current normalized by the electrochemically active surface area (ECSA) of the electrode. This facilitates activity comparisons between catalysts with different roughness factors (RF, defined here as the ratio of ECSA/geometric electrode area), allowing one to determine if higher catalytic activity results from a higher average turnover frequency or simply from an increased number of active sites. There exist a variety of methods in electrocatalysis for determining the ECSA based on capacitance, under-potential deposition, CO stripping, and redox features that can be employed depending on the surface chemistry and morphology, and the best method varies based on the material studied. So far, only capacitance has found wide use for estimating the ECSA of nanostructured polycrystalline copper electrodes..$^{90-96}$ We emphasize that on any non-planar surface, the measurement of the ECSA is at best an approximation; consequently, we recommend that researchers take the limitations of each method of determining the ECSA into account when analyzing their data. Furthermore, we would also like to highlight the need for all future work, particularly on nanostructured electrodes, to report the ECSA whenever possible. It is only possible to evaluate whether a novel catalyst represents an improvement in intrinsic activity compared to state-of-the-art materials if the ECSA-normalized activity can be compared with the literature. As will be shown in Subsection 6.2.4 and Section 7.4, much of the suggested activity improvements in the field can attributed to increased surface area. 


\subsection{Intrinsic Kinetics vs. Mass Transport Effects}

As a consequence of the HER and $\mathrm{CO}_{2} \mathrm{R}$, which deplete protons or (equivalently) produce hydroxide ions, a $\mathrm{pH}$ gradient can develop at the electrode surface. This high local $\mathrm{pH}$ has several competing effects on these reactions due to a complicated interplay between mass transport, buffer equilibria, and $\mathrm{pH}$, which will be discussed in more detail in Subsection 3.3.1. Notably, in the $\mathrm{CO}_{2} /$ bicarbonate system, $\mathrm{CO}_{2}$ is both a reactant and a buffer, which means that changes in $\mathrm{pH}$ near the cathode surface can cause the concentration of dissolved $\mathrm{CO}_{2}$ to deviate from the concentration in the bulk electrolyte. For planar polycrystalline $\mathrm{Cu}$, it was found that the reported activity at potentials more cathodic than $-1 \mathrm{~V} v s$. RHE is strongly influenced by concentration polarization and thus does not represent its true intrinsic activity under bulk pH 6.8 conditions ${ }^{97}$ However, this cannot be used as a universal rule of thumb, as the hydrodynamics of the electrochemical cell and the morphology of the catalyst itself can both impact the extent of concentration polarization at a given potential.

Ultimately, since both the HER and $\mathrm{CO}_{2} \mathrm{R}$ produce one equivalent of $\mathrm{OH}^{-}$for each $\mathrm{e}^{-}$transfer (with the exception of the organic anions formate and acetate, for which one less $\mathrm{OH}^{-}$is released than electron transferred), changes in interfacial concentrations are primarily a function of the electrolyte and the total geometric current density,. This means that nanostructured catalysts with high surface areas will be more susceptible to concentration polarization at lower overpotentials due to the increased activity on a geometric area basis and increased transport requirements within a porous catalyst compared to a planar electrode..$^{98,99}$ Therefore, caution must be taken when comparing $\mathrm{CO}_{2} \mathrm{R}$ performance between catalysts with very different roughness factors since they will reach mass transport limitations at different rates.

In general, it is critical to ensure sufficient supply of $\mathrm{CO}_{2}$ to the electrode surface. In aqueous systems, this is accomplished by diffusion of $\mathrm{CO}_{2}$ through the electrolyte, so it is important that the electrochemical cell is well-mixed in order to maximize the potential window where the reaction is governed by intrinsic reaction kinetics. As one would expect for a diffusion-limited process, it has been shown that the maximum rate of $\mathrm{CO}_{2} \mathrm{R}$ increases with increased mixing due to a decrease in the boundary layer thickness. ${ }^{100-103}$ Thus, the hydrodynamics of an electrochemical cell will also have a 
direct effect on the local $\mathrm{pH}$ change at the electrode surface. We therefore recommend that researchers quantify the boundary layer thickness, for example, by measuring the diffusion-limited current density for a facile, outer sphere redox reaction such as ferricyanide reduction. ${ }^{88}$ This will allow for better comparisons between different electrochemical cells and mass transport conditions. Still, as described further in Subsection 3.3.1, correcting for mass transport effects in aqueous systems is extremely difficult. As a result, we strongly recommend that conclusions about intrinsic activity and selectivity only be drawn from data that is not impacted by significant concentration polarization so as not to convolute effects of the catalyst properties and the effects of mass transport.

\subsection{Deactivation of $\mathrm{Cu}$ Electrodes}

It has been demonstrated that the activity and selectivity of polycrystalline and single crystalline $\mathrm{Cu}$ electrodes towards $\mathrm{CO}_{2} \mathrm{R}$ and $\mathrm{COR}$ decays over time, although the details vary. ${ }^{104-118}$ The changes in activity can be tentatively attributed to (i) poisoning by impurities from the experimental setup, (ii) poisoning of the surface with reaction intermediates, and/or (iii) restructuring of the surface under reaction conditions. The latter will be discussed in Subsection 3.1.3, as it has been observed with insitu/operando methods that the $\mathrm{Cu}$ surface structure is dynamic and can reconstruct under reaction conditions. More often, the deactivation is attributed either to deposition of metal contaminants from the electrolyte, ${ }^{112,114} \mathrm{CO}_{2}$ stream, ${ }^{113}$ glassware, ${ }^{115}$ or counter electrode, ${ }^{119}$ including $\mathrm{Fe},{ }^{112,113} \mathrm{Zn},{ }^{112,114}$ $\mathrm{Pb},{ }^{114} \mathrm{Si},{ }^{115}$ and $\mathrm{Pt} ;{ }^{119}$ or to poisoning or coking of the surface by (decomposition of) reaction intermediates. ${ }^{104-111,118}$ Authors have recommended using the highest-purity reagents, ${ }^{112,113}$ preelectrolyzing the electrolyte solution, ${ }^{112}$ and/or removing impurities from the electrolyte with a bed of chelating agent before use ${ }^{114}$ to minimize the impact of contaminants on the reaction. Additionally, in light of the observation that $\mathrm{Pt}$ from the counter electrode can deposit on the working electrode despite separation by an anion exchange membrane, the use of alternate counter electrodes has also been suggested. ${ }^{88,119}$ Deactivation does not seem to play as much of a role in high surface area electrodes (this has mechanistic implications that will be discussed in Chapter 5)..$^{88,110,115}$ 
Finally, it is worth mentioning another technique that has been used to prevent deactivation and/or restore activity for $\mathrm{CO}_{2} \mathrm{R}$ on $\mathrm{Cu}$, namely, anodic pulses or sweeps. ${ }^{105,109,111,118,120}$ Pulsed electrolysis may allow for anodic stripping of poisoning species from the electrode surface. ${ }^{111}$ Anodic pulses or sweeps will also likely cause roughening of the electrode and/or oxidation of the $\mathrm{Cu}$ surface. ${ }^{109,118,120,121}$ Gupta et al. modeled the effect of pulsed electroreduction and showed that pulsing causes corresponding swings in local $\mathrm{pH}$ and $\mathrm{CO}_{2}$ concentrations. ${ }^{100}$ While we cannot say with certainty which of these factors are responsible, it is clear that pulsing or sweeping to anodic potentials has a beneficial impact on maintaining and/or restoring $\mathrm{CO}_{2} \mathrm{R}$ activity on $\mathrm{Cu}$. There have also been several other interesting observations noted when using potential pulsing/sweeping techniques, such as the formation of methanol ${ }^{122}$ and increased selectivity for the production of $\mathrm{C}_{2+}$ compounds. ${ }^{101,120,123}$ These results have been attributed to transient changes in the local chemical conditions such as an increased coverage of $* \mathrm{OH}$ and an accumulation of $\mathrm{CO}$ near the $\mathrm{Cu}$ surface, respectively. On the other hand, studies using applied voltage pulses in the millisecond time regime demonstrated that the selectivity on polycrystalline $\mathrm{Cu}$ could be shifted entirely to syngas $\left(\mathrm{H}_{2}\right.$ and $\left.\mathrm{CO}\right)$, with the ratio of these two products changing with the length of the pulses, ${ }^{121}$ or to strongly favor $\mathrm{CO}$ or $\mathrm{CH}_{4}$ production. ${ }^{124}$ Since these pulsed-bias methods have yielded increased selectivities for desirable products in addition to helping prevent deactivation, further work should be done to better understand the mechanisms at play and how they can best be utilized to enhance $\mathrm{CO}_{2} \mathrm{R}$ catalysis.

\subsection{Deconvolution of Various Effects in Complex Catalysts}

In addition to the deactivation mechanisms just discussed, there are a number of other factors that can lead to discrepancies in the catalytic performance of $\mathrm{Cu}$ between electrochemical systems. For example, as we will discuss in Section 3.3, the choice of electrolyte anions, cations, and $\mathrm{pH}$ can have a significant influence on the catalyst performance, so ideally, direct comparisons should only be made between catalysts tested in the same electrolytes. Ultimately, the entire electrochemical system (including catalyst, electrolyte composition \& concentration, electrochemical cell geometry \& hydrodynamics, membrane composition, and operating conditions) needs to be considered in order to 
determine if the measured activity and selectivity can be confidently attributed to the properties of the catalyst itself. To ensure that experimental conditions are satisfactory, researchers should benchmark their electrochemical set-up by making sure that literature results using a standard electrode material, such as planar polycrystalline $\mathrm{Cu}$, can be reproduced (within the variation typical of the field, as indicated by Figure 4) If this is not the case, any results obtained from more advanced materials are not meaningful, as other factors could be impacting the catalysis.

As will be discussed further in Chapters 6 and 7, different preparation methods can also yield very different $\mathrm{CO}_{2} \mathrm{R}$ performance for seemingly similar catalysts. Unfortunately, many advanced catalyst designs such as nanostructured and/or bimetallic electrodes are ill-defined, which can make it extremely challenging to deconvolute the influence of different morphological factors that can cause changes in behavior (such as surface faceting, composition, oxidation state, strain, grain boundaries, porosity, etc.). Complex catalysts should preferably be characterized to the greatest extent possible. In general, however, planar and/or well-defined electrodes are best for systematic studies that seek to draw fundamental relationships between activity/selectivity and structure/composition of catalysts. Furthermore, detailed studies as a function of voltage are needed to properly identify when novel catalysts are limited by their intrinsic kinetics rather than a convolution of kinetic and mass transport effects; only reporting data at one potential gives an incomplete picture of the catalyst behavior and can complicate comparisons between different catalysts and/or electrochemical systems.

Another key challenge in this area is the disconnect between $e x$-situ characterization and how catalysts function in-situ. This is especially challenging for a highly mobile and readily oxidized material such as $\mathrm{Cu}$. We caution against drawing conclusions about the catalyst under reaction conditions using ex-situ measurements alone, since the electrolyte and negative polarization of the cathode can have a significant influence on the catalyst properties. We also urge the continued development of in-situ/operando spectroscopic techniques in order to elucidate the true chemical state and structure of active sites during electrochemical $\mathrm{CO}_{2} \mathrm{R}$. Not only will this aid in the search for more active and selective catalysts, but this can also provide more useful insights into the reaction mechanisms occurring on the surface on an atomic-scale level. 


\section{Experimental Probes of $\mathrm{CO}_{2}$ Reduction Mechanisms}

In Section 1.2 we discussed how a large number of $>2 \mathrm{e}^{-}$products are formed during $\mathrm{CO}_{2} \mathrm{R}$ on polycrystalline $\mathrm{Cu}$ electrodes. Steering selectivity towards certain products instead of others is therefore an important challenge, and could potentially be done by stabilizing/destabilizing key intermediates, making the pathway of the desired product(s) energetically more favorable. Thus, comprehensive mechanistic understanding of $\mathrm{CO}_{2} \mathrm{R}$ is crucial in order to design more selective catalysts. In this chapter, various experimental studies aiming to provide mechanistic insight about $\mathrm{CO}_{2} \mathrm{R}$ on $\mathrm{Cu}$ electrodes are presented. This includes measurements on single crystals, further reduction of possible intermediates, and measurements probing the impact of the electrolyte and electrochemical conditions. The key findings will furthermore be the basis of the theoretical descriptions of the processes occurring on $\mathrm{Cu}$ surfaces, which will be discussed in detail in Chapter 4. Then, Chapter 5 will bring together the key conclusions from these experimental and theoretical studies and present our current understanding of the complex reaction networks for electrochemical $\mathrm{CO}_{2} \mathrm{R}$ on $\mathrm{Cu}$.

We would first like to acknowledge a growing area of research that underpins much of the gains in mechanistic understanding: in-situ/operando spectroscopies. Development of spectroanalytic techniques, such as X-ray absorption spectroscopy (XAS), ${ }^{125-128}$ Raman spectroscopy ${ }^{96,129-137}$ and various forms of infrared (IR) spectroscopy, ${ }^{119,134,137-150}$ has become increasingly important for discerning the catalyst surface structure, composition, and oxidation state under in-situ/operando conditions, as well for detecting the presence of adsorbed intermediates. Therefore, we will highlight relevant studies where they have contributed to mechanistic understanding throughout the different sections of this work. 


\subsection{The Influence of Surface Facet on $\mathrm{CO}_{2}$ and $\mathrm{CO}$ Reduction}

It is complicated, if not impossible, to derive unambiguously which structural elements present on a polycrystalline catalyst surface are contributing to the observed catalytic activity, since the measured catalytic activity is always a sum of activities resulting from different structures. In a simplified picture, a catalyst surface can consist of planar areas with single crystal orientations and various step and kink sites with low coordination. Further, complex atomic structures could be present at the interface between different grains that are typically observed in polycrystalline materials and/or nanostructured surfaces. $\mathrm{Cu}$ single crystal electrodes with preferential orientation are possible model catalysts to investigate the first two of the mentioned structural properties. This includes planar surfaces, such as $\mathrm{Cu}(111), \mathrm{Cu}(100)$ and $\mathrm{Cu}(110)$, as well as higher-index surfaces which can be described as a low-index surface with a certain density of step sites. Single crystal electrodes are ideal systems to draw comparisons with theoretical calculations because methods such as density functional theory (DFT) only probe local phenomena, which should be equally distributed on these atomically flat catalysts. Further, single crystals are also quite useful for studying the influence of other $\mathrm{CO}_{2} \mathrm{R}$ rate-determining factors such as electrolyte $\mathrm{pH}$, cations and anions since they provide a well-defined surface structure. Note, however, that single crystals themselves are not viable catalysts for practical applications, due to their low geometric surface area, and the resulting low geometric current densities. Therefore, several groups have synthesized nanostructured materials with preferential faceting and high dispersion, which will be discussed further in Chapter 6. In this section, we describe experimental findings obtained on single-crystal model systems, describe surface reconstructions on $\mathrm{Cu}$, discuss the mechanistic interpretations, and suggest focus areas for future studies.

\subsubsection{Influence of Various Cu(hkl) Surfaces on Product Distribution}

The electrochemical reduction of $\mathrm{CO}_{2}$ on $\mathrm{Cu}$ single crystals has been studied by a variety of methods, including voltammetry (linear potential sweeps), ${ }^{151-156}$ chronoamperometry (constant potential) ${ }^{155,157,158}$ and chronopotentiometry (constant current). ${ }^{151-154,159}$ The choice of electrochemical technique depends on the method employed to characterize liquid and gaseous products. For instance, 
in-situ techniques that can monitor the evolution of products in real-time, such as differential electrochemical mass spectrometry (DEMS) can be coupled with cyclic voltammetry (CV) or other scanning techniques. However, ex-situ bulk product analysis, such as chromatography techniques and NMR spectroscopy, need to be coupled with chronoamperometry and/or chronopotentiometry. The choice of technique also depends on whether quantitative information, such as the concentrations of products formed, is of interest, or if it is sufficient to identify trends between electrodes.

Early chronopotentiometric measurements by Frese indicated that among the three low-index $\mathrm{Cu}$ single crystal facets, i.e, $\mathrm{Cu}(111), \mathrm{Cu}(110)$ and $\mathrm{Cu}(100)$, the $\mathrm{Cu}(111)$ surface yields the highest $\mathrm{CH}_{4}$ rate and $\mathrm{Cu}(100)$ the lowest. ${ }^{159}$ Further measurements by Hori et al. indicated that $\mathrm{Cu}(100)$ surfaces primarily form $\mathrm{C}_{2} \mathrm{H}_{4}$ and a somewhat smaller amount of $\mathrm{CH}_{4}$, while $\mathrm{Cu}(110)$ and $\mathrm{Cu}(111)$ surfaces preferentially form $\mathrm{CH}_{4}$ with very small amounts of $\mathrm{C}_{2} \mathrm{H}_{4} \cdot{ }^{151}$ This was later confirmed in follow-up measurements by Hori et al., ${ }^{152-154}$ as well as other research groups. ${ }^{155,156}$ In a study showing the product distribution over a range of potentials, $\mathrm{C}_{2} \mathrm{H}_{4}$ formation set in at a similar or lower overpotential than $\mathrm{CH}_{4}$ formation for all investigated single crystal electrodes. ${ }^{156}$ At higher overpotentials, the formation of $\mathrm{CH}_{4}$ becomes the dominant process. ${ }^{156}$ This is similar to the trend observed for polycrystalline $\mathrm{Cu}$, as shown in Figure 4 of Subsection 1.2.2, for which $\mathrm{CH}_{4}$ formation reaches maximum selectivity at around $-1.1 \mathrm{~V}$ to $-1.2 \mathrm{~V} v s$. RHE, where $\mathrm{CO}_{2} \mathrm{R}$ activity starts becoming mass transport limited on planar electrodes. ${ }^{66,97,156}$

Liquid, oxygenated compounds are interesting products as well, due to their aforementioned high energy density and moderate to high value. In the previously described studies by Hori et al., several liquid compounds were detected, of which alcohols and carboxylates were the most significant, as shown in Table 4. ${ }^{152-154}$ Around $-1.0 \mathrm{~V}$ vs. RHE, $\mathrm{Cu}(100)$ surfaces preferentially formed alcohols compared to formic acid, while the opposite could be observed for $\mathrm{Cu}(111) . \mathrm{Cu}(110)$ surfaces yielded similar selectivity towards alcohols and carboxylates. Huang et al. recently showed a similar trend as Hori et al. around $-1.0 \mathrm{~V} v s$. RHE, and less cathodic potentials, the main liquid product observed was formate. ${ }^{156}$ At potentials cathodic of $-1.0 \mathrm{~V} v s$. RHE, where the formation of $\mathrm{C}_{2} \mathrm{H}_{4}$ reached a maximum on all single crystal surfaces, Huang et al. observed formation of ethanol with significant Faradaic 
efficiency. A similar trend was observed on polycrystalline $\mathrm{Cu}$, suggesting that the two products are formed through a partially shared pathway.

Table 4: Product distribution in $\mathrm{CO}_{2}$ reduction at a series of copper single-crystal electrodes, $\mathrm{Cu}(\mathrm{S})$ $[\mathrm{n}(100) \mathrm{x}(111)]$ and $\mathrm{Cu}(\mathrm{S})-[\mathrm{n}(100) \mathrm{x}(110)]$. The measurements were performed by chronopotentiometriy at $5 \mathrm{~mA} \mathrm{~cm}{ }^{-2}$ in $0.1 \mathrm{M} \mathrm{KHCO}_{3}$. The potentials $v s$. RHE were calculated from the potentials $v s$. SHE using the Nernst equation, assuming a $\mathrm{pH}$ of 6.8. Adapted with permission from Hori. ${ }^{1}$ Copyright (C) 2008, Springer Science Business Media, LLC. The data for $\mathrm{Cu}(110)$ is from Hori, 1995. ${ }^{151}$

\begin{tabular}{|c|c|c|ccccccc|c|}
\hline $\begin{array}{c}\text { Crystal } \\
\text { orientation }\end{array}$ & $\begin{array}{c}\text { Potential / } \\
\text { V vs. SHE }\end{array}$ & $\begin{array}{c}\text { Potential / } \\
\text { V vs. RHE }\end{array}$ & $\mathbf{C H}_{\mathbf{4}}$ & $\mathbf{C}_{2} \mathbf{H}_{\mathbf{4}}$ & $\mathbf{C O}$ & Alc. & Ald. & $\mathbf{H C O O H}$ & $\mathbf{H}_{2}$ & $\mathbf{C}_{2} \mathbf{H}_{4} /$ \\
$\mathbf{C H}_{4}$
\end{tabular}

In order to elucidate the influence of undercoordinated sites on the product distribution, Hori et al. also performed a series of $\mathrm{CO}_{2}$ reduction studies on stepped $\mathrm{Cu}$ single crystal surfaces. ${ }^{152-154}$ The distribution of gaseous and liquid products is summarized in Table 4. The presence of (111) or (110) steps on $\mathrm{Cu}(100)$ surfaces led to significantly enhanced Faradaic efficiency toward alcohols. Their data also indicated that a number of stepped surfaces exist that yield higher $\mathrm{C}_{2} \mathrm{H}_{4} / \mathrm{CH}_{4}$ ratios compared to the $\mathrm{Cu}(100)$ surface, which is the most selective low-index crystal facet for the formation of $\mathrm{C}_{2} \mathrm{H}_{4}$, as described above. In Figure 5, the $\mathrm{C}_{2} \mathrm{H}_{4} / \mathrm{CH}_{4}$ ratio is plotted as a function of crystal orientation, clearly illustrating the notion that steps can further enhance the selectivity to $\mathrm{C}_{2} \mathrm{H}_{4}$ formation compared to the planar single crystals. Thus, it seems that one approach to improving the selectivity towards $\mathrm{C}_{2} \mathrm{H}_{4}$ is to engineer $\mathrm{Cu}$ electrodes with an increased occurrence of (100) surfaces and optimal occurrence of steps. Indeed, this has been seen in epitaxially-grown copper films with a high degree of preferential surface orientation, where films with a preferentially (751)-oriented surface rich in steps with highly 
undercoordinated sites show high selectivity to $\mathrm{C}_{2+}$ products and especially oxygenated products. ${ }^{158}$ Additional efforts following this direction will be discussed in more detail in Chapter 6 .

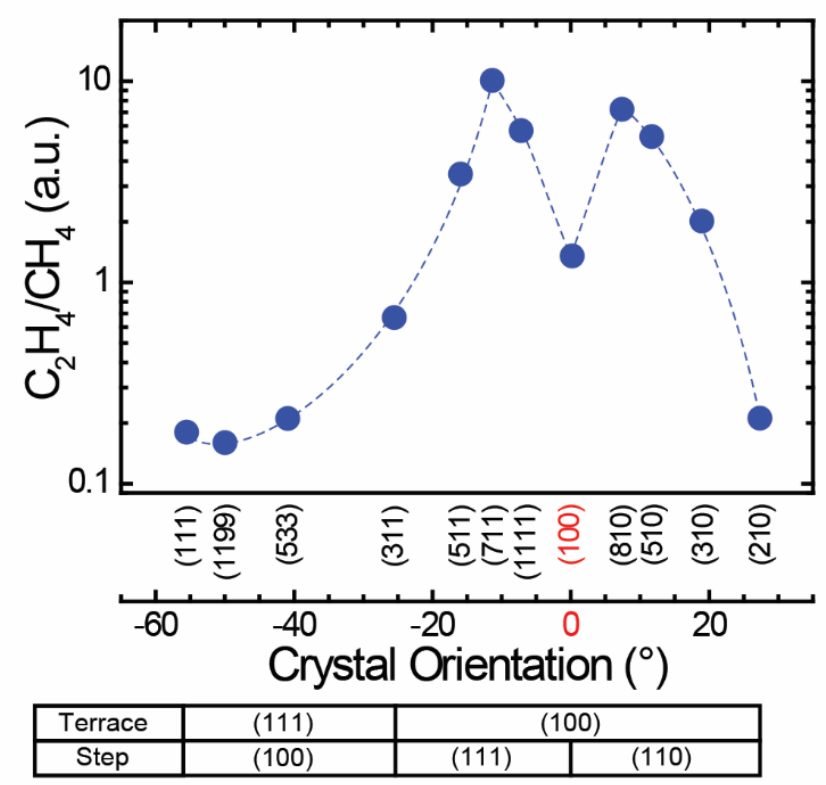

Figure 5: Variation of $\log \left(\mathrm{C}_{2} \mathrm{H}_{4} / \mathrm{CH}_{4}\right)$ in terms of the current efficiency and the electrode potential with the angle of the crystal orientation with reference of $\mathrm{Cu}(100)$. The data for the figure were obtained from Hori et al. ${ }^{154}$

As discussed later in Subsections 3.2.1 and 4.2.2, $\mathrm{CO}$ has been identified as a key intermediate in the reduction of $\mathrm{CO}_{2}$ to $>2 \mathrm{e}^{-}$products. ${ }^{86,104,160-162}$ Thus, to obtain further mechanistic insight about the formation of these compounds, COR has been studied on $\mathrm{Cu}$ single crystals. ${ }^{163-167}$ None of these studies quantified the various products formed, making it hard to draw conclusions about Faradaic efficiencies and partial current densities. However, studying the onset potentials for the various products can give an indication of the selectivity towards the different compounds in various potential ranges. In DEMS measurements of various single crystals in phosphate buffer at $\mathrm{pH} 7, \mathrm{Cu}(100)$ exhibited an earlier onset than $\mathrm{Cu}(111)$ and $\mathrm{Cu}(110)$ for $\mathrm{C}_{2} \mathrm{H}_{4} \cdot{ }^{167}$ Further, $\mathrm{Cu}(100)$ facilitated little to no $\mathrm{CH}_{4}$ formation at the measured potentials, while $\mathrm{Cu}(111)$ gave a similar onset for $\mathrm{C}_{2} \mathrm{H}_{4}$ and $\mathrm{CH}_{4} \cdot \mathrm{Cu}(911)$, which was shown by Hori et al. to be particularly selective towards $\mathrm{C}_{2} \mathrm{H}_{4}$, behaves similarly to $\mathrm{Cu}(100)$ in this study. In order to gain more conclusive insights about COR on various single crystalline surfaces, further systematic investigations with complete product quantification are required, similar to what has been demonstrated by Hori et al. for $\mathrm{CO}_{2}$ reduction. 
Based on the discussion above, it is clear that $\mathrm{CO}_{2} \mathrm{R}$ measurements on single crystals can yield important information about which surface sites are responsible for the formation of key products. Increasing our understanding of the active surface can aid in the development of highly selective catalyst materials. However, based on the measurements described in the previous paragraphs, it seems clear that more work performing thorough and complete product analysis is necessary before conclusions can be drawn about specific trends. In addition, the reports on non-planar surfaces indicate that engineering of materials to achieve presence of well-defined steps on the surface can be a valuable design principle for improving selectivity toward $\mathrm{C}_{2+}$ products such as $\mathrm{C}_{2} \mathrm{H}_{4}$ and alcohols.

\subsubsection{Influence of Various Cu(hkl) Surfaces on Activity}

As will be discussed in Chapter 4, theoretical studies predict vastly different activity for different crystal facets, rather than just the selectivity differences described so far. In order to be able to evaluate $\mathrm{CO}_{2} \mathrm{R}$ activity experimentally, thorough, quantitative product analysis is needed. Additionally, the catalyst needs to be studied while under activation control, i.e., not experiencing mass transport limitations, as discussed in detail in Section 2.2. As a result, only a few of the reports mentioned in the previous subsection can be used to draw conclusions about trends in activity. These studies ${ }^{154,156,158}$ do show slight variations in both overall and $\mathrm{CO}_{2} \mathrm{R}$ activity depending on orientation, but nothing comparable to the magnitude expected based on computational studies. For example, the study on epitaxially grown $\mathrm{Cu}$ thin films reported $\sim 1$ order of magnitude higher activity towards $\mathrm{C}-\mathrm{C}$ coupled products for the highly stepped $\mathrm{Cu}(751)$-oriented surface as compared to the planar $\mathrm{Cu}(111)$ oriented surface. ${ }^{158}$ These results are illustrated in Figure 6. Note that while these electrodes exhibit preferential orientation, they are not truly single crystalline, meaning that they have a certain occurrence of grain boundaries and other undercoordinated defects on the surface. This could explain why the activity of these and other well-defined surfaces do not show as much difference as predicted by theory. Step sites have been identified as the main contributor to the activity of various catalytic systems. For instance, it has been seen in the field of thermally activated catalysis that blocking step sites can have a drastic impact; experiments where the steps of Ru single crystals ( $1 \%$ of the surface) were blocked led to a reduction in $\mathrm{N}_{2}$ dissociation activity by more than 9 orders of magnitude. ${ }^{168}$ Similarly, a small 
fraction of step sites have also been suggested to dominate the overall electrochemical $\mathrm{CO}_{2} / \mathrm{CO}$ reduction activity. ${ }^{169}$ Thus, even a small quantity of existing defects or step sites formed in-situ (vide infra) could be dominating the activity of these otherwise single crystalline electrodes.

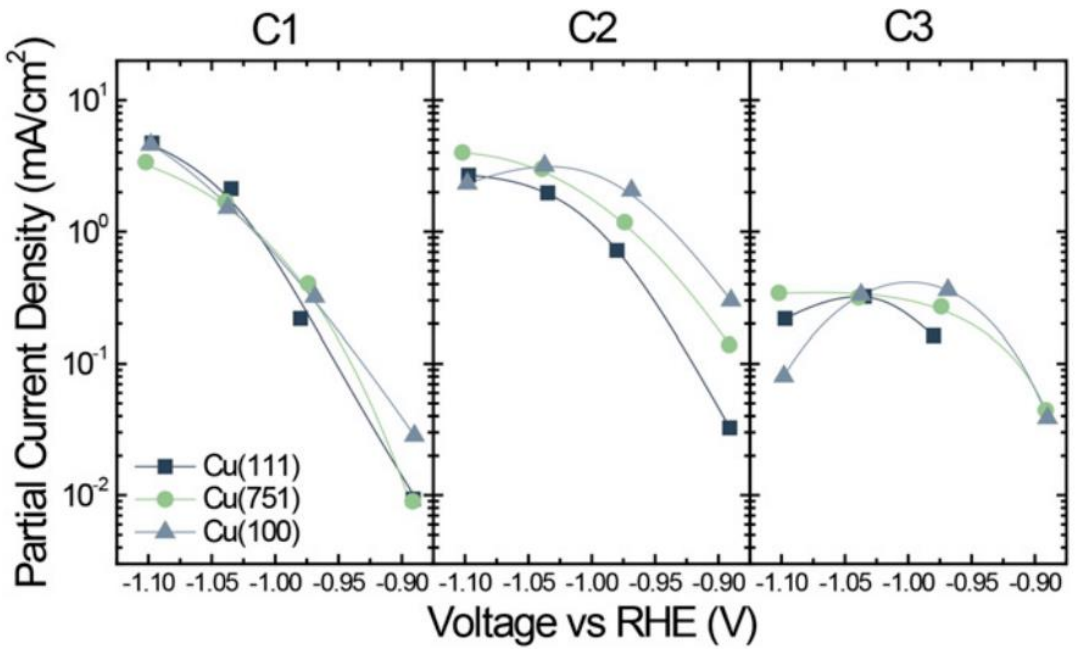

Figure 6: $\mathrm{CO}_{2} \mathrm{R}$ on epitaxially grown $\mathrm{Cu}$ films oriented in the (111), (751) and (100) directions. The graphs show partial current density toward $\mathrm{C}_{1}$ (left), $\mathrm{C}_{2}$ (middle) and $\mathrm{C}_{3}$ (right) products. Reprinted from Hahn et al. ${ }^{158}$

\subsubsection{Restructuring and Other Challenges Regarding Single Crystal Measurements}

As described above, single crystal measurements can be highly useful to study the $\mathrm{CO}_{2} \mathrm{R}$ activity and selectivity of various structures. However, one must be cautious because the quality and consistency of single crystal studies are sensitive to a number of factors, discussed herein. Hori et al. described in detail how the reproducibility of $\mathrm{CO}_{2} \mathrm{R}$ measurements on $\mathrm{Cu}(110)$ and $\mathrm{Cu}(111)$ single crystals strongly depends on the pre-treatment of the electrodes. ${ }^{153}$ Although the structural properties of the bulk are well-defined, the homogeneity of the surface can vary depending on the pre-treatment. One possible factor could be the formation of oxides during the preparation and transfer to the electrochemical cell. A partially oxidized surface, which is reduced in the actual electrochemical cell, could lead to an irreversible reconstruction of the $\mathrm{Cu}$ surface, a phenomenon that for example has been shown in detail for Pt electrodes. ${ }^{170,171}$ This effect has also been intentionally exploited to prepare nanostructured electrodes in a number of studies, as discussed in Subsection 6.2.1. As a result of the oxidation occurring upon exposure to ambient air, Hori and coworkers do not rule out that the final 
electrodes might be textured with defect sites, which could have a large influence on the measured electrocatalytic activity of the electrodes, as we just discussed. However, they probed the surface structure only by the use of $\mathrm{CV}$, which can give indicative but not conclusive evidence.

Surface probe techniques, such as scanning tunneling microscopy (STM), can image the surface with excellent resolution to evaluate whether defect sites are present. It is important to keep in mind that only small fractions of the electrode surface are probed with this type of technique, so the resulting image may therefore not provide a representative indication of the whole surface. Nonetheless, STM can give a good impression of the defect density for various types of electrodes. In Figure 7, STM images are shown from 4 different preferentially oriented electrodes, spanning 3 preparation procedures and 4 measurement conditions. Figure 7a shows an STM image of a $\mathrm{Cu}(100)$ single crystal that was prepared by annealing under ultra-high vacuum (UHV) and transferred to the characterization chamber without being exposed to oxygen. The crystal exhibits large terraces and a relatively small amount of defects on the surface. In comparison, in-situ STM images of electropolished $\mathrm{Cu}(111)($ Figure 7b) and $\mathrm{Cu}(100)$ (Figure 7c) show significant occurrence of steps and other defects. An example of in-situ STM images from an epitaxially grown $\mathrm{Cu}(100)$-oriented film are shown in Figure 7d, which also display a significant occurrence of defects since this preparation procedure does not lead to truly single crystalline electrodes. 

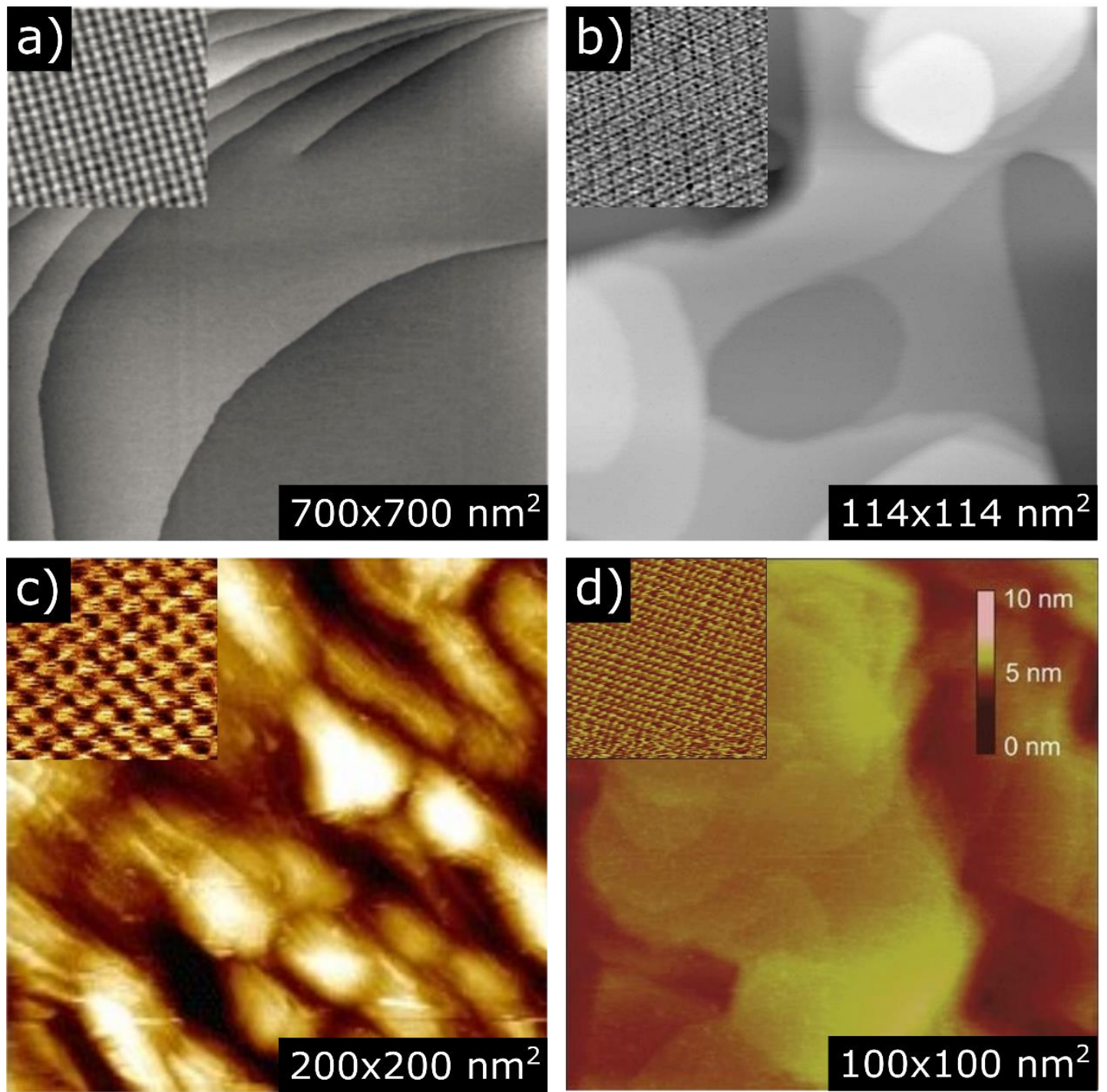

Figure 7: STM images of various oriented surfaces. (a) $700 \mathrm{~nm}$ x $700 \mathrm{~nm}$ STM image of ultra-high vacuum prepared $\mathrm{Cu}(100)$ single crystal. Inset: $4 \mathrm{~nm}$ x $4 \mathrm{~nm}$ image of the same surface. Reproduced with permission from Engstfeld et al. ${ }^{172}$ Copyright (C) 2018, John Wiley and Sons. (b) $114 \mathrm{~nm}$ x $114 \mathrm{~nm}$ in-situ STM image of electropolished $\mathrm{Cu}(111)$ single crystal at $-0.6 \mathrm{~V}$ (vs. pseudo-reference) in $\mathrm{PO}_{4}$ buffer. Inset: $9.5 \mathrm{~nm} \times 9.5 \mathrm{~nm}$ image of the same surface. Reprinted with permission from Schlaup et al. ${ }^{173}$ Copyright (C) 2012, Elsevier B.V. (c) $200 \mathrm{~nm}$ x $200 \mathrm{~nm}$ in-situ STM image of electropolished $\mathrm{Cu}$ (100) single crystal at $-0.9 \mathrm{~V}$ vs. SHE. Inset: $2 \mathrm{~nm} \times 2 \mathrm{~nm}$ image of the same surface. Adapted with permission from Kim et al. ${ }^{174}$ Copyright $@$ 2016, Springer Science Business Media New York. (d) 100 $\mathrm{nm} \times 100 \mathrm{~nm}$ in-situ STM image of epitaxially grown $\mathrm{Cu}(100)$ film at $-0.24 \mathrm{~V} v$ s. RHE in $0.1 \mathrm{M} \mathrm{HClO}_{4}$. Inset: $5 \mathrm{~nm} \times 5 \mathrm{~nm}$ image of the same surface. Reprinted from Hahn et al. ${ }^{158}$

In addition to the influence of pre-treatment, there is the possibility that $\mathrm{Cu}$ surface restructuring under reaction conditions can lead to changes in activity and selectivity. Earlier reports indicated that $\mathrm{Cu}(111)$ restructures to a highly textured surface when exposed to even small $\mathrm{CO}$ partial pressures in vacuum, ${ }^{175}$ and recent experimental spectroscopic ${ }^{134}$ and computational ${ }^{176}$ studies have also indicated that the presence of $\mathrm{CO}$ increases the density of highly active, undercoordinated step sites on 
polycrystalline $\mathrm{Cu}$. Furthermore, restructuring promoted by $\mathrm{CO}$ was also observed using operando grazing incidence X-ray diffraction (GIXRD) on polycrystalline copper thin films. ${ }^{177}$ Thus, since there is typically a significant coverage of $\mathrm{CO}$ under $\mathrm{CO}_{2} \mathrm{R}$ conditions, ${ }^{178}$ it is possible that reconstruction and the formation of step sites on otherwise defect-free single crystalline surfaces during reaction could explain why there are only moderate differences in $\mathrm{CO}_{2} \mathrm{R}$ activity on different facets, as mentioned above.

Various studies employing in-situ electrochemical scanning tunneling microscopy (ECSTM) have also reported reconstruction of $\mathrm{Cu}$ surfaces under applied potentials while not in the presence of $\mathrm{CO}_{2} / \mathrm{CO} \cdot{ }^{173,174,179-182}$ For example, it has been demonstrated that at a potential of $-0.9 \mathrm{~V} v s$. SHE in both argon-purged $0.1 \mathrm{M} \mathrm{KOH}$ and $0.1 \mathrm{M} \mathrm{KHCO}_{3}$, a polycrystalline $\mathrm{Cu}$ surface gradually forms $\mathrm{Cu}(111)$ facets on a timescale of 30 minutes, and subsequently transform into $\mathrm{Cu}(100)$ facets after another 30 minutes. ${ }^{179,182} \mathrm{The} \mathrm{Cu}(110)$ surface undergoes a similar restructuring compared to the polycrystalline $\mathrm{Cu}$ electrodes; the top-most planes of a $\mathrm{Cu}(110)$ surface transformed over time into a disordered $\mathrm{Cu}(110)$, then into a disordered mixed $\mathrm{Cu}(110)-\mathrm{Cu}(111)$, and finally into an ordered $\mathrm{Cu}(110)-\mathrm{Cu}(100)$ plane. ${ }^{181}$ In contrast, no reconstruction was observed on single crystal $\mathrm{Cu}(111)$ or $\mathrm{Cu}(100)$ surfaces under the same conditions, ${ }^{181}$ nor for epitaxially grown $\mathrm{Cu}(111)$ and $\mathrm{Cu}(100)$ films at $-0.24 \mathrm{~V}$ vs. RHE in $0.1 \mathrm{M} \mathrm{HClO}_{4}{ }^{158}$ While it is somewhat unclear what drives the (in)stability of these different systems, it is likely that it has to do with the comparative adhesions of various surfaces in contact with each other. ${ }^{181}$ Another possibility could be due to other factors from the local reaction environment (i.e., electrolyte and/or potential-dependent effects). However, more work needs to be done to better understand how and why $\mathrm{Cu}$ restructuring occurs under reaction conditions.

Another important point for $\mathrm{Cu}$ is that, compared to other monometallic catalyst systems, ${ }^{170}$ few thorough benchmarking studies on the electrochemical properties of $\mathrm{Cu}$ single crystal surfaces have been reported. Such data could be used to determine the properties of electrodes used for a particular study, which is standard practice for other electrode materials, such as Pt. ${ }^{183}$ For example, CVs of single crystal electrodes recorded in alkaline solutions at potentials where only $\mathrm{OH}$ adsorption on the surface occurs (-0.2 V to $0.5 \mathrm{~V} v s$. RHE) and no bulk oxide formation is expected, exhibit different redox features for different reports in the literature, which should not be the case for truly single crystal 
electrodes. ${ }^{156,165,180,184-187}$ Further, a recent study on the electrochemical properties of polycrystalline $\mathrm{Cu}$ and $\mathrm{Cu}(100)$ electrodes, including samples prepared under UHV conditions to avoid surface contamination of oxygen, clearly suggests that the features observed in the CV strongly depend on the pre-treatment of the electrodes. ${ }^{172}$ This is illustrated in Figure 8, where the $\mathrm{CV}$ of a $\mathrm{Cu}$ electrode prepared under UHV conditions is plotted $v s$. the CVs obtained for samples exposed to different pretreatments under laboratory conditions. ${ }^{172}$ The authors ascribed the features in the CV between $0.30 \mathrm{~V}$ and $0.45 \mathrm{~V}$ to different sites than the $\mathrm{Cu}(100)$ planes, indicating that all electrodes except for that prepared under UHV conditions contain a significant amount of defect sites on the surface. Other approaches to assess the consistency of $\mathrm{Cu}$ electrodes could include comparing key values for the HER, which so far has only been reported in a few studies on polycrystalline $\mathrm{Cu}$ electrodes in alkaline ${ }^{188-190}$ or acidic ${ }^{191}$ solutions, or investigating the distinct features obtained from lead ${ }^{191,192}$ or thallium ${ }^{193}$ underpotential deposition (UPD) on single crystal electrodes.

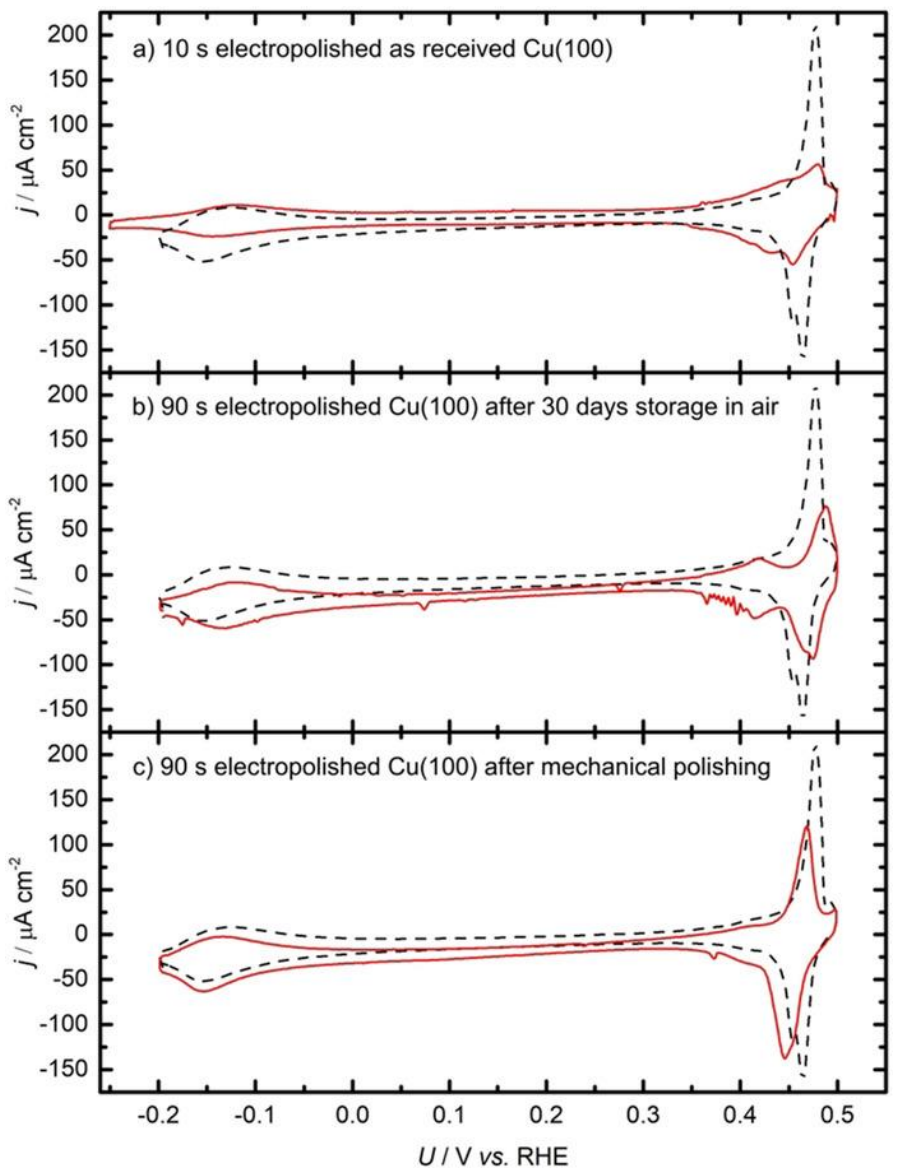

Figure 8: Comparison of the $\mathrm{CV}$ recorded on a $\mathrm{Cu}(100)$ electrode prepared under UHV conditions (dashed line) with a) an electropolished as received $\mathrm{Cu}(100)$ electrode from the supplier, b) $90 \mathrm{~s}$ electropolished $\mathrm{Cu}(100)$ electrode after storage in air for 30 days and $\mathbf{c})$ an electropolished $\mathrm{Cu}(100)$ 
electrode after mechanical polishing $(0.1 \mu \mathrm{m}$ Alumina powder for 10 minutes and further 10 minutes with $0.05 \mu \mathrm{m}$ Alumina powder). All CVs were recorded in $0.1 \mathrm{M} \mathrm{KOH}$ and at a scan rate of $50 \mathrm{mV} \cdot \mathrm{s}^{-}$

${ }^{1}$. Reprinted with permission from Engstfeld et al. ${ }^{172}$ Copyright $@$ ( 2018, John Wiley and Sons.

Better insight about the fundamental electrochemical properties of $\mathrm{Cu}$ single crystal electrodes would be valuable in order to better compare results among different research groups within the field of $\mathrm{CO}_{2} \mathrm{R}$. In addition, it would be useful to elucidate the influence of higher overpotentials and different electrolytes and $\mathrm{pH}$ ranges. For example, it was shown by $\mathrm{CV}$ that polycrystalline $\mathrm{Cu}$ electrodes exposed to $-0.5 \mathrm{~V}$ or $-0.6 \mathrm{~V} v s$. RHE in $1 \mathrm{M} \mathrm{NaOH}$ at $60{ }^{\circ} \mathrm{C}$ for 3 hours that new sites are formed on the surface. ${ }^{194}$ Cathodic corrosion, hydrogen embrittlement or hydride formation can also play a crucial role at such potentials. ${ }^{195,196}$ These effects have received little attention in relation to $\mathrm{CO}_{2} \mathrm{R}$ as of yet, but could influence activity and stability. Furthermore, such insights could also be highly useful for other processes where the electro-chemical/-catalytic properties of $\mathrm{Cu}$ are important, such as nitrate reduction or studies related to the initial oxidation of $\mathrm{Cu}$ single crystal electrodes in aqueous environment. ${ }^{197-200}$

\subsection{Reduction of Possible Intermediates}

In the previous section, we outlined the facet-dependent activity \& selectivity of $\mathrm{Cu}$ and saw how tunable the reaction is on the atomic scale. Here we will discuss some of the key intermediate species and how probing their reactivity has helped shed light on $\mathrm{CO}_{2}$ reduction pathways.

\subsubsection{CO as a Crucial Intermediate}

Carbon monoxide $(\mathrm{CO})$ and formate $\left(\mathrm{HCOO}^{-}\right)$are the $2 \mathrm{e}^{-}$products formed from $\mathrm{CO}_{2} \mathrm{R}$, and as discussed in the Subsection 1.2.2, there are classes of metals that yield each of these products with high selectivity. Early experimental work found that the reduction of $\mathrm{CO}$ on $\mathrm{Cu}$ leads to a similar product distribution as $\mathrm{CO}_{2} \mathrm{R}$ on $\mathrm{Cu}$, suggesting that $\mathrm{CO}$ is a key reaction intermediate. ${ }^{86,104,160-162}$ Subsequent detailed studies of $\mathrm{CO}$ reduction (COR) on $\mathrm{Cu}$ have provided further support for this claim, ${ }^{115,160,201}$ in addition to the spectroscopic observation of surface adsorbed $\mathrm{CO}$ under $\mathrm{CO}_{2} \mathrm{R}$ conditions. ${ }^{133-135,145-148}$ Consequently, several studies proposed that the binding energy of adsorbed $\mathrm{CO}\left({ }^{*} \mathrm{CO}\right)$ is a key 
descriptor for reduction activity to $>2 \mathrm{e}^{-}$products. ${ }^{202-204}$ Metals that bind $\mathrm{CO}$ too strongly will be poisoned by this intermediate, whereas metals that bind $\mathrm{CO}$ too weakly will desorb it from the surface before it can react further. Thus, well-aligned with the Sabatier principle, ${ }^{205}$ the intermediate binding energy for $\mathrm{CO}$ on $\mathrm{Cu}$ is a key reason for its ability to catalyze $\mathrm{CO}_{2} \mathrm{R}$ to products further reduced than CO. In addition, studies have shown that $\mathrm{CO}$ adsorption on $\mathrm{Cu}$ suppresses the competing hydrogen evolution reaction (HER) due to site blocking effects and/or changes in $* \mathrm{H}$ binding energy. ${ }^{86,178,202,206}$ The coverage of $\mathrm{CO}$ on $\mathrm{Cu}$ is typically very high, ${ }^{178}$ at least until there are mass transport limitations (vide infra). Thus, $\mathrm{CO}$ poisoning of the HER is an important mechanism by which $\mathrm{Cu}$ maintains a high Faradaic efficiency for $\mathrm{CO}_{2} \mathrm{R}$ in aqueous electrolytes. ${ }^{206}$

Ever since the determination of $\mathrm{CO}$ as a key intermediate in the reduction of $\mathrm{CO}_{2}$ to hydrocarbons and alcohols, there has been increasing interest in studying COR as a proxy for understanding trends in $\mathrm{CO}_{2} \mathrm{R}$. This has several advantages including fewer reaction steps/intermediates to consider and avoiding the complication of the reactant being part of the buffer (as is the case for $\mathrm{CO}_{2} \mathrm{R}$ in bicarbonate solutions). The latter, in turn, allows for a wider range of $\mathrm{pH}$ conditions to be examined, in addition to allowing for direct comparison with blank measurements where the electrolyte is purged with an inert gas. Another motivation for better understanding COR catalysis is that this could provide an alternate route for $\mathrm{CO}_{2} \mathrm{R}$ that can potentially overcome scaling relations by splitting the reaction into two separate steps: 1) $\mathrm{CO}_{2}$ reduction to $\mathrm{CO}$, then 2) $\mathrm{CO}$ reduction to the desired product(s). Thus, we will discuss insights gained from experimental and computational COR studies where appropriate in the sections that follow, and we also recommend continued development of viable COR catalysts for potential tandem systems.

\subsubsection{Reduction of Oxygenated $\mathrm{C}_{1}$ and $\mathrm{C}_{2}$ Species}

The discovery of at least 16 distinct products formed from $\mathrm{CO}_{2} \mathrm{R}$ on $\mathrm{Cu}$ highlights the complexity of this reaction. ${ }^{66}$ Besides the dominant hydrocarbons, methane and ethylene, this includes a broad mix of aldehydes, ketones, carboxylic acids, and alcohols, the majority of which are multicarbon compounds. As a result, probing the reduction activity of small oxygenated species has been 
used as a technique to help elucidate mechanistic pathways; here we will briefly describe the key findings from these studies.

Unlike $\mathrm{CO}$, it has been shown that another $2 \mathrm{e}^{-}$reduction product, formate/formic acid, does not typically undergo further reduction on $\mathrm{Cu}$ under reductive conditions; ${ }^{86}$ however with very high concentrations, high cathodic bias, and highly acidic or basic electrolytes, trace amounts of methane have been reported. ${ }^{104,207}$ Formaldehyde $\left(\mathrm{CH}_{2} \mathrm{O}\right)$ reduction has been shown to produce mainly methanol, ${ }^{108,113,160}$ although small amounts of methane have also been reported. ${ }^{104,108,113,160,207}$ Finally, reduction of methanol (or methoxide, which is methanol deprotonated under high $\mathrm{pH}$ ) has been shown to yield no products. ${ }^{104,113,207}$ These results strongly suggest that formate and methanol are terminal products for electrochemical $\mathrm{CO}_{2} \mathrm{R}$, and intermediate(s) related to formaldehyde (such as adsorbed formyl $* \mathrm{CHO}$ ) may be on the pathway to methanol and methane.

The presence of hydroxyl and/or carbonyl moieties in many of the multi-carbon products suggests that the $\mathrm{C}-\mathrm{C}$ bond is formed early in the reaction, at least before one of the two carbon-oxygen bonds in $\mathrm{CO}_{2}$ is broken. ${ }^{66}$ Accordingly, it has been suggested that enol-like species may be intermediates to multi-carbon products, ${ }^{66}$ such as an enediol or enediolate. ${ }^{113}$ The observation that reduction of ethylene oxide produces ethylene indicates that a strongly adsorbed epoxide in the form of an oxametallacycle is another possible intermediate in ethylene formation. ${ }^{113}$ Ethylene glycol and oxalic acid reduction did not lead to any reduction products, suggesting that these are not intermediates in $\mathrm{CO}_{2} \mathrm{R} \cdot{ }^{113}$ This is curious given that ethylene glycol has been detected as a minor product, ${ }^{66}$ so a recent theoretical study suggested that the reason why this species is not further reduced is due to its $\mathrm{OH}$ groups being repelled by the surface, making its re-adsorption endergonic. ${ }^{208}$ Finally, both glyoxal ${ }^{113,209}$ and glycoaldehyde ${ }^{113}$ reduction have been shown to produce acetaldehyde and ethanol but not ethylene. This suggests that these minor products are intermediates on the pathway to acetaldehyde and ethanol. ${ }^{208}$

It has long been thought that aldehydes are key intermediates to alcohols after Hori and coworkers observed that acetaldehyde and propionaldehyde were easily reduced to ethanol and npropanol, respectively. ${ }^{160}$ Further support for this has been provided by several COR studies that have noted aldehyde production at lower overpotentials than alcohols that decreases with increasing 
overpotential where alcohols become dominant, ${ }^{115,201}$ as well as recent demonstrations of direct acetaldehyde reduction to ethanol. ${ }^{91,210}$ Another study employed a DEMS set-up where the catalyst was coated directly onto the pervaporation membrane used to transfer volatile species into the mass spectrometer in order to detect species directly from the electrode-electrolyte interface. ${ }^{101}$ Through this method, it was observed that acetaldehyde and propionaldehyde are produced in 2-4 times higher abundance than ethanol or propanol compared to sampling from the bulk electrolyte, where they are typically only observed as minor products. ${ }^{101}$ Thus, the authors suggest that these aldehydes are transiently produced \& relatively abundant within the local reaction environment, but are typically reduced further to the corresponding alcohols before diffusing into the bulk electrolyte. Interestingly, it was also found that ethanol production increased at the expense of propionaldehyde at more cathodic potentials, suggesting that ethanol and propionaldehyde may share a common intermediate such as acetaldehyde. ${ }^{101}$

Finally, it is worth highlighting a recent study that has led to significant new insights on the role of water in the formation of $\mathrm{C}_{2+}$ oxygenated products. ${ }^{211}$ By carrying out $\mathrm{C}^{16} \mathrm{O}$ reduction in $\mathrm{H}_{2}{ }^{18} \mathrm{O}$ electrolyte and quantifying the isotopic composition of the products using gas chromatography-mass spectrometry (GC-MS), Lum et al. determined that the majority of ethanol, acetate, and 1-propanol formed were ${ }^{18} \mathrm{O}$ enriched, meaning that they incorporated oxygen from the solvent water. Allyl alcohol and methanol were not enriched with ${ }^{18} \mathrm{O}$, which suggests different mechanisms for their formation. Importantly, water has not previously been considered as a dominant $\mathrm{O}$ source in the formation of oxygenated products, so the researchers then investigated how this could be the case using quantum mechanics-based metadynamics calculations. This led to the identification of a new mechanism for $\left({ }^{18} \mathrm{O}\right)$ ethanol formation, which was termed as Grotthuss chain ethynyl concerted hydrolysis $(\mathrm{GECH}) .{ }^{211}$ The mechanistic pathways and intermediate branch points for formation of ethylene, $\left({ }^{18} \mathrm{O}\right)$ ethanol, and $\left({ }^{16} \mathrm{O}\right)$ ethanol were then elucidated; these $\mathrm{C}_{2}$ reaction networks are shown in purple in Figure 18. It is important to keep in mind that Cannizzaro-type disproportionation reactions could also occur in the electrolyte, especially at high $\mathrm{pH}$, converting an aldehyde (acetaldehyde) into the corresponding alcohol (ethanol) and carboxylate (acetate). ${ }^{212}$ Thus, this is another possible mechanism by which ${ }^{18} \mathrm{O}$ could be 
incorporated into the products. Lum et al. performed control experiments that indicate this is not the main mechanism by which these products are formed, ${ }^{211}$ but researchers should still keep the possibility of these reactions in mind when working under strongly alkaline conditions, especially when a high concentration of aldehydes are formed.

\subsection{Electrolyte Effects}

The choice of electrolyte can have a huge impact on $\mathrm{CO}_{2} \mathrm{R}$ performance. There are a number of factors that have complex relations with the local reaction environment; the composition and concentration of anions \& cations can cause changes in the electrostatic interactions, buffer capacity, $\mathrm{pH}$, and availability of proton donors. These changes are not always straightforward to map due to intertwining effects. In this section we will discuss the major variables within the electrolyte $(\mathrm{pH}$, cations, anions) and their impacts on $\mathrm{CO}_{2} \mathrm{R}$ catalysis.

\subsection{1 pH Effects}

Studying the effect of $\mathrm{pH}$ on $\mathrm{CO}_{2} \mathrm{R}$ comes with several challenges. Given that $\mathrm{CO}_{2}$ forms bicarbonate and carbonate when it is purged through alkaline solutions, electrolytes for $\mathrm{CO}_{2} \mathrm{R}$ are generally limited to neutral and acidic $\mathrm{pH}$ conditions. However, by using $\mathrm{CO}$ reduction (COR) as a proxy, mechanistic insights about the pathway to further reduced products can be obtained. Pioneering work of this nature by Hori et al. first revealed that the selectivity between $\mathrm{CH}_{4}$ and $\mathrm{C}_{2} \mathrm{H}_{4}$ is impacted by $\mathrm{pH} \cdot{ }^{86,160,161}$ By studying $\mathrm{COR}$ on $\mathrm{Cu}$, the production rates for each product could be compared in buffer solutions spanning a bulk pH of 6 to $12 .{ }^{160}$ When the measurements of partial current density towards $\mathrm{C}_{2} \mathrm{H}_{4}\left(\right.$ or $\left.\mathrm{C}_{2} \mathrm{H}_{5} \mathrm{OH}\right)$ are included on a single Tafel plot on the SHE scale (Figure 9a), a straight line emerges. Thus, the rate-determining step on the pathway to $\mathrm{C}_{2+}$ products such as $\mathrm{C}_{2} \mathrm{H}_{4}$ is $\mathrm{pH}$ independent on an absolute potential scale (SHE), and thus will shift on the RHE scale to more positive potential (decreasing overpotential) by $59 \mathrm{mV}$ per unit increase in $\mathrm{pH}$. $\mathrm{For}_{\mathrm{CH}}$, on the other hand, the Tafel plot was widely scattered unless only measurements at the same $\mathrm{pH}$ were included, indicating that 
the rate-determining step for the $\mathrm{C}_{1}$ pathway involves proton transfer and is thus dependent on $\mathrm{pH}$. Hori et al. explained this $\mathrm{pH}$ dependence in terms of the Tafel equation where the partial current for $\mathrm{CH}_{4}$ is proportional to the proton activity; indeed, by plotting the logarithm of the $\mathrm{CH}_{4}$ partial current density plus pH (Figure 9b), a straight line emerges. From these plots, transfer coefficients of $\alpha=0.35$ and $\alpha=$ 1.33 were extracted for $\mathrm{C}_{2} \mathrm{H}_{4}$ and $\mathrm{CH}_{4}$, respectively. ${ }^{160}$ This led Hori et al. to propose that the reaction paths for $\mathrm{CH}_{4}$ and $\mathrm{C}_{2} \mathrm{H}_{4}$ formation do not share rate-determining steps and thus have mechanisms that are separated at early stages of $\mathrm{CO}$ reduction. Further evidence for this notion was provided in a series of studies on single crystal electrodes by the group of Marc Koper, ${ }^{164,166,167}$ which revealed that there are two possible pathways to $\mathrm{C}_{2} \mathrm{H}_{4}$ depending on the $\mathrm{pH}$, surface structure and overpotential. These mechanisms will be discussed further in Chapters 4 and 5.
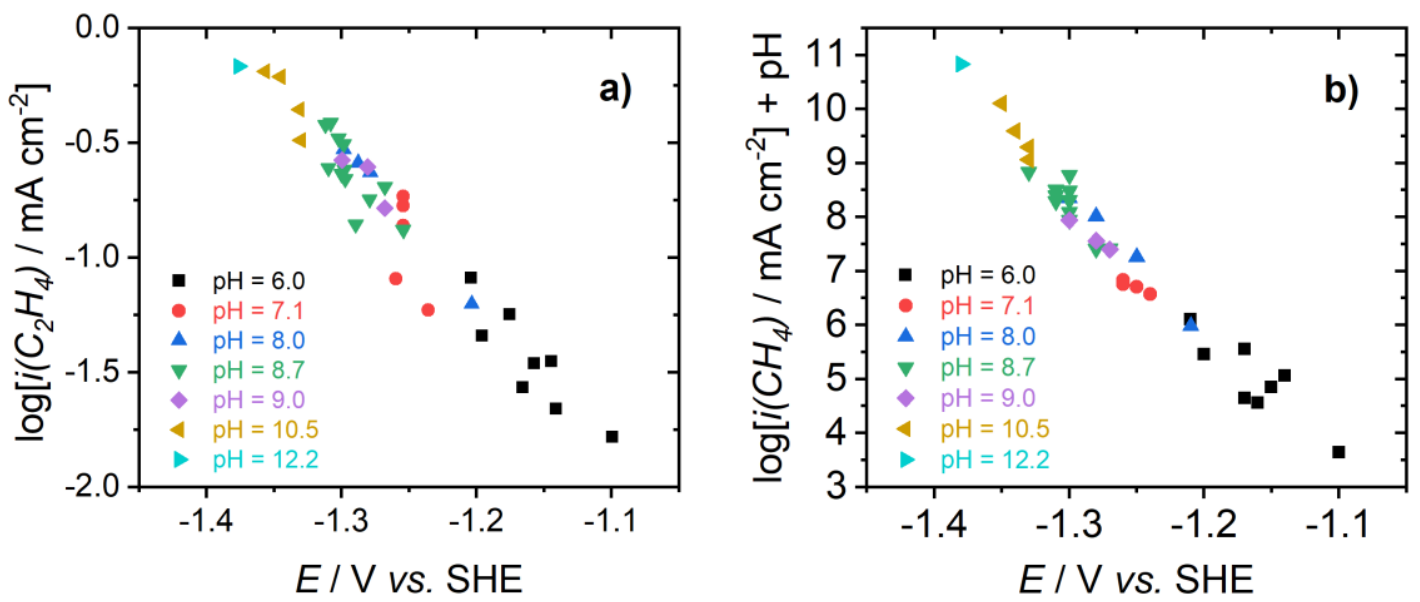

Figure 9: Tafel plots from COR measurements that were made over a $\mathrm{pH}$ range of 6 to 12. a) plots the logarithm of the partial current density going towards ethylene against applied potential on the SHE scale; b) plots the logarithm of the partial current density going towards methane, plus the $\mathrm{pH}$, against applied potential on the SHE scale. Adapted from Hori et al. ${ }^{160}$ Copyright $\odot 1997$, American Chemical Society.

Recent work comparing $\mathrm{CO}_{2} \mathrm{R}$ and $\mathrm{COR}$ on planar polycrystalline $\mathrm{Cu}$ in $\mathrm{pH} 6.8$ and 13 , respectively, has led to increased mechanistic understanding of the role of $\mathrm{pH} .^{201}$ The partial current densities for $\mathrm{CO}_{2} \mathrm{R}$ and COR products plotted on both the RHE and SHE scale illustrates the overall effect of $\mathrm{pH}$ (Figure 10). The $\mathrm{C}_{2+}$ product overpotentials display a $\mathrm{pH}$ independence on the SHE scale, essentially overlapping for $\mathrm{CO}_{2} \mathrm{R}$ and $\mathrm{COR}$, which corresponds to a positive $\sim 0.36 \mathrm{~V}$ shift on the $\mathrm{RHE}$ 
scale from $\mathrm{pH} 6.8$ to $\mathrm{pH} 13$ (or $\sim 59 \mathrm{mV}$ per $\mathrm{pH}$ unit, as expected). On the other hand, the $\mathrm{C}_{1}$ product $\left(\mathrm{CH}_{4}\right)$ overpotential shows a $\mathrm{pH}$ dependence on the SHE scale, leading to a much smaller shift on the RHE scale of $\sim 0.15 \mathrm{~V}$. It was concluded that both field stabilization of a rate-limiting chemical reaction (i.e. $\mathrm{CO}$ dimerization) or proton-electron transfer from water during the rate-limiting step are plausible explanations for the $\mathrm{pH}$ independence of the $\mathrm{C}_{2+}$ pathway on the SHE scale. Then, the complex $\mathrm{pH}$ dependence for $\mathrm{CH}_{4}$ was rationalized by a kinetic analysis, which suggested that this pathway is limited by a later proton-electron transfer. ${ }^{201,213}$ These findings, which will be discussed in more detail in Section 4.2 and in the SI, are in good agreement with Hori's observations (see Section 1 of the SI for a derivation of the RHE-scale $\mathrm{pH}$ dependence from Figure 9b). Notably, because the overpotential for $\mathrm{C}_{2+}$ products is greatly reduced on the RHE scale, this means that operating in basic electrolytes can lead to a major improvement in energy efficiency for the production of multi-carbon compounds from $\mathrm{CO}_{2} \mathrm{R} / \mathrm{COR}$. Consequently, $\mathrm{KOH}$ electrolytes are increasingly being explored as a means to achieve higher selectivity and energy efficiency for $\mathrm{C}_{2+}$ products, particularly in vapor-fed systems where $\mathrm{CO}_{2}$ is directly delivered in the gas-phase rather than solubilized in the aqueous electrolyte and diffused to the cathode. These efforts are described in more detail in Section 6.3. 

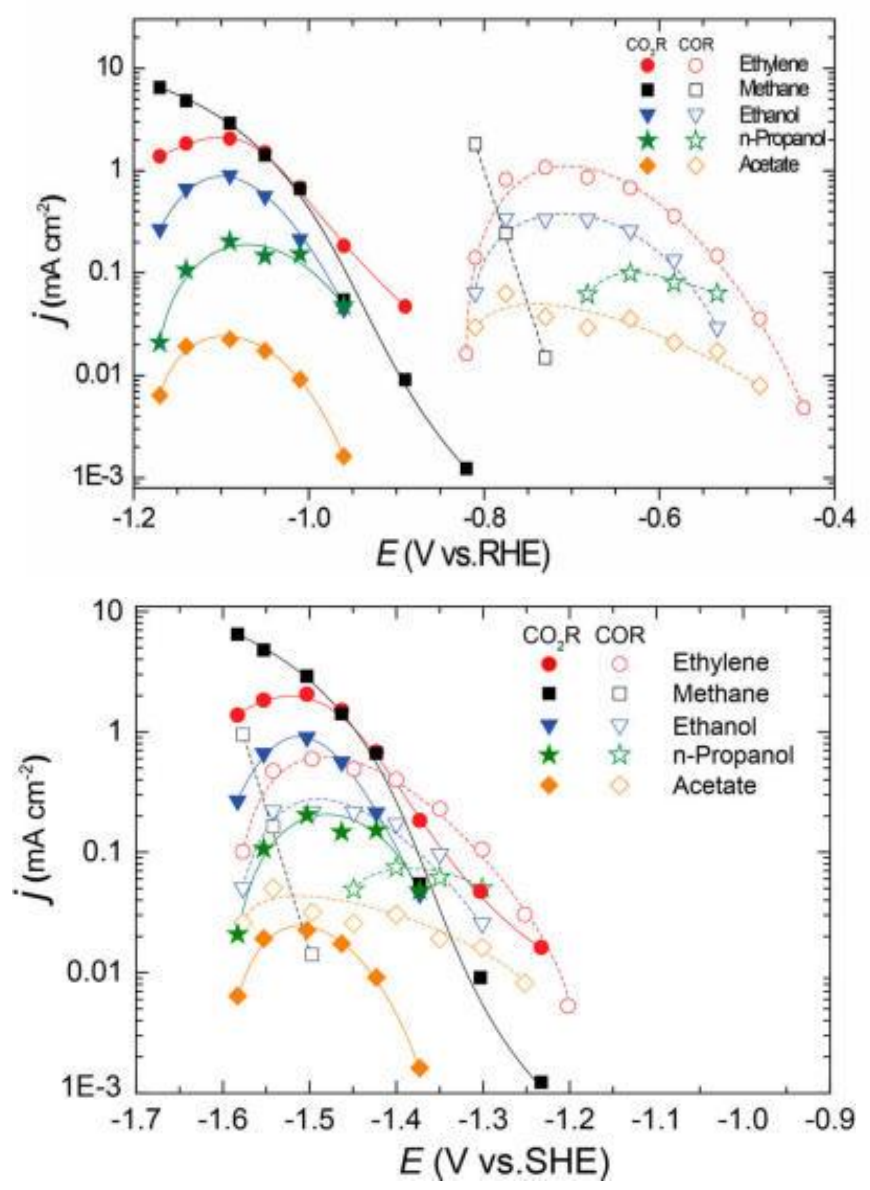

Figure 10: $\mathrm{CO}_{2} \mathrm{R}$ (filled) and COR (hollow) partial current densities on the RHE scale (top) and the SHE scale (bottom). Reprinted from Wang et al. ${ }^{201}$ Copyright $\odot$ 2018, American Chemical Society

While $\mathrm{CO}_{2} \mathrm{R}$ has not typically been conducted in aqueous electrolytes with high $\mathrm{pH}$ due to the buffering capacity of $\mathrm{CO}_{2}$, understanding the impacts of $\mathrm{pH}$ has still been a major area of study due to the dynamic reaction environment at the electrode surface. As discussed in Section 2.2, a local $\mathrm{pH}$ gradient can develop at the cathode surface due to depletion of protons/production of hydroxide ions from the HER and $\mathrm{CO}_{2} \mathrm{R}$. In the $\mathrm{CO}_{2}$ /bicarbonate system, $\mathrm{CO}_{2}$ is both a reactant and a buffer, so an increased $\mathrm{pH}$ near the cathode surface can cause the concentration of dissolved $\mathrm{CO}_{2}$ to deviate (decrease) from that in the bulk electrolyte. The key equilibria reactions for this system are: ${ }^{2,100,214}$

$$
\begin{aligned}
& \mathrm{CO}_{2(\mathrm{~g})} \leftrightarrow \mathrm{CO}_{2(\mathrm{aq})} \\
& \mathrm{CO}_{2(\mathrm{aq})}+\mathrm{H}_{2} \mathrm{O} \leftrightarrow \mathrm{H}_{2} \mathrm{CO}_{3} \\
& \mathrm{H}_{2} \mathrm{CO}_{3} \leftrightarrow \mathrm{HCO}_{3}^{-}+\mathrm{H}^{+}
\end{aligned}
$$

$$
\begin{aligned}
& K_{S}=30 \mathrm{mM} / \mathrm{bar} \\
& K_{e q}=1.7 \times 10^{-3} \\
& p K_{a}=3.6
\end{aligned}
$$




$$
\begin{array}{lll}
\mathrm{CO}_{2(\mathrm{aq})}+\mathrm{H}_{2} \mathrm{O} \leftrightarrow \mathrm{HCO}_{3}^{-}+\mathrm{H}^{+} & (11=11.1+11.2) & p K_{a}=6.4 \\
\mathrm{HCO}_{3}^{-} \leftrightarrow \mathrm{CO}_{3}^{2-}+\mathrm{H}^{+} & (12) & p K_{a}=10.3
\end{array}
$$

Or in alkaline solutions, these can be written as:

$$
\begin{aligned}
& \mathrm{CO}_{2}+\mathrm{OH}^{-} \leftrightarrow \mathrm{HCO}_{3}^{-} \\
& \mathrm{HCO}_{3}^{-}+\mathrm{OH}^{-} \leftrightarrow \mathrm{CO}_{3}^{2-}+\mathrm{H}_{2} \mathrm{O}
\end{aligned}
$$

Reactions 10,11 , and 12 result in $\mathrm{CO}_{2}$ saturated solutions having bulk $\mathrm{pH}$ values from around pH 6-8 for bicarbonate concentrations of $0.05-2 \mathrm{M}$, respectively. ${ }^{2}$ The kinetics of $\mathrm{CO}_{2}$ hydration (Reaction 11.1) are slow, and the equilibrium concentration of $\mathrm{H}_{2} \mathrm{CO}_{3}$ is very low. ${ }^{2,100}$ However, the nucleophilic attack of dissolved $\mathrm{CO}_{2}$ by $\mathrm{OH}^{-}$is faster, such that Reaction 11a dominates over Reaction $11(11=11.1+11.2)$ when $\mathrm{pH}>10 .^{214}$ The kinetics for the protonation/deprotonation reactions $(11.2$, 12, and 12a) are all quite fast, and it can be assumed that $\mathrm{HCO}_{3}{ }^{-}$is in equilibrium with $\mathrm{CO}_{3}{ }^{2-}$ at all times. ${ }^{100}$ Mathematical models have been used to solve the relevant diffusion equations, along with the reaction equations for $\mathrm{CO}_{2}$ electroreduction with a given selectivity and geometric current density, to estimate the interfacial $\mathrm{pH}$ and concentrations, many of which are based on the seminal work by Gupta et al. (ref $\left.{ }^{100}\right){ }^{86,97-100,110,215-218}$ It has been shown that even modest current densities can cause the interfacial $\mathrm{pH}$ to vary significantly from that in the bulk electrolyte, perturbing the reaction thermodynamics and kinetics in several ways.

First, it is worth taking a moment to briefly discuss the impact of $\mathrm{pH}$ on the HER. At higher $\mathrm{pH}$ where the proton concentration is low, water reduction is expected to dominate over proton reduction. From a thermodynamics standpoint, HER is pH-independent on the RHE scale and it is irrelevant whether the reaction proceeds via proton or water reduction; in principle, any Brønsted acid can act as a proton donor. ${ }^{206}$ However, this can have a huge impact on the kinetics of the HER, as demonstrated by Strmcnik et al. for noble-metal electrodes. ${ }^{219,220}$ Furthermore, Grozovski et al. distinguished $\mathrm{H}^{+}$ reduction from $\mathrm{H}_{2} \mathrm{O}$ reduction and found their corresponding kinetic parameters using a combination of RDE experiments and modeling. ${ }^{221}$ It is expected that the HER proceeds primarily via water reduction under $\mathrm{CO}_{2} \mathrm{R}$ conditions. ${ }^{206}$ It is also possible that the electrolyte buffer can act as a proton donor, 
depending on its $\mathrm{pKa}$ value, concentration, and mass transport/availability; this will be discussed in more detail in Subsection 3.3.3. While the explicit impact of $\mathrm{pH}$ on the HER is still an ongoing area of research, it has been thought that increasing local $\mathrm{pH}$ helps favor $\mathrm{CO}_{2} \mathrm{R}$ over $\mathrm{HER}$, mainly due to the decreasing overpotential for the formation of $\mathrm{C}_{2+}$ products, as discussed earlier. On the other hand, a high local $\mathrm{pH}$ shifts equilibria of the acid-base reactions towards (bi)carbonates, which can reduce the concentration of $\mathrm{CO}_{2}$ near the surface, decreasing selectivity towards $\mathrm{CO}_{2} \mathrm{R}$ and favoring the HER instead. ${ }^{97,215}$

These contradictory consequences have led to some debate over whether a high local $\mathrm{pH}$ is beneficial or detrimental for $\mathrm{CO}_{2} \mathrm{R}$ catalysis. Several researchers have proposed that $\mathrm{CO}_{2}$ is still likely to be present under a non-equilibrium high $\mathrm{pH}$ owing to its slow hydration kinetics. ${ }^{86,139,150,222,223}$ This of course is very much dependent on the sufficient transport of $\mathrm{CO}_{2}$ to the surface. However, with this in mind, it seems likely that there is an optimum $\mathrm{pH}$ that balances the benefits of increased $\mathrm{pH}$ with the slow, disadvantageous decline in $\mathrm{CO}_{2}$ concentration. Indeed, recent modeling studies have found such a volcano-type dependence on $\mathrm{pH}$ for $\mathrm{CO}_{2} \mathrm{R}$ selectivity (particularly to $\mathrm{C}_{2+}$ products) and proposed an optimal local $\mathrm{pH}$ range of 9-10.98,99 At higher $\mathrm{pHs}$, the HER becomes dominant due to mass transport limitations of $\mathrm{CO}_{2}$. Interestingly, in this mass transport limited regime, the activity and selectivity for $\mathrm{C}_{2+}$ products plateaus and eventually declines, while $\mathrm{CH}_{4}$ production generally increases. ${ }^{66,97,201}$ Thus, one can identify when the reaction is limited by $\mathrm{CO}_{2}$ mass transport by observing when the $\mathrm{C}_{2+}$ activity plateaus ${ }^{201}$ or by increasing mixing at a given potential to see if the $\mathrm{C}_{2+}$ selectivity is altered. ${ }^{209} \mathrm{An}$ example of the latter approach is shown in Figure 11. While it remains unclear how pH itself affects the adsorption energies and coverages of reaction intermediates, especially since these are all also a strong function of potential, it seems likely that $* \mathrm{H}$ coverage increases at the expense of $* \mathrm{CO}$ in this mass transport limited regime, providing a stronger driving force for hydrogenation of $\mathrm{CO}$ to $\mathrm{CH}_{4}$ and a decreased probability of $\mathrm{CO}$ coupling to $\mathrm{C}_{2+}$ products. ${ }^{66,216}$ This hypothesis was further supported by a recent study incorporating microkinetic modeling, which revealed that $* \mathrm{CO}$ coverage is gradually depleted as its reduction becomes increasingly favorable with decreasing (more cathodic) potential, and 
formation rates of $\mathrm{C}_{2+}$ products are more drastically impacted due to their second-order dependence on $*$ CO coverage. ${ }^{213}$

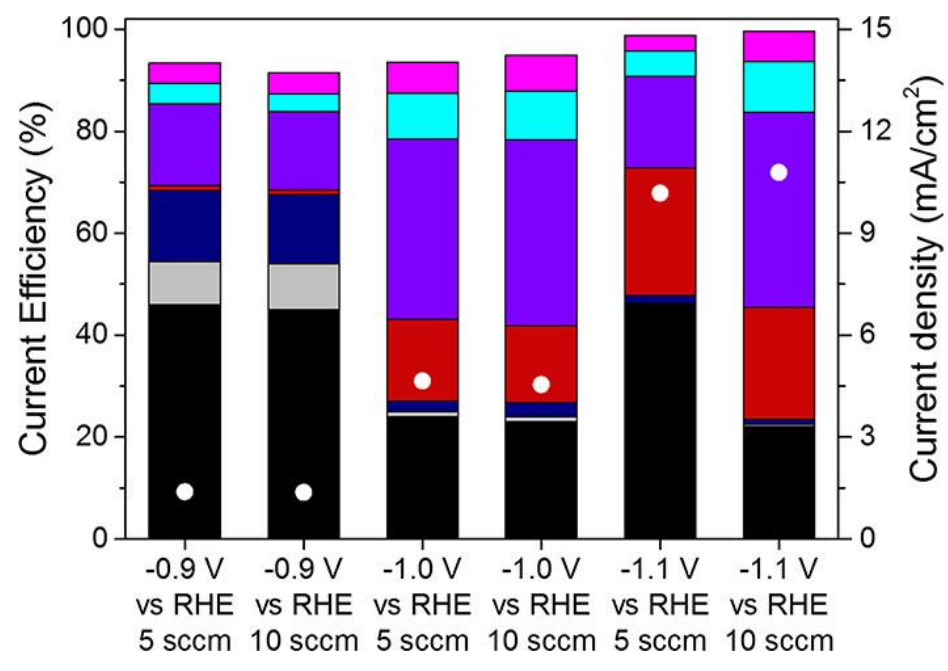

\author{
Hydrogen \\ Carbon monoxide \\ Formate \\ Methane \\ Ethylene \\ Ethanol \\ Minor liquid products
}

Figure 11: Current efficiency (bars) and total current density (dots) for $\mathrm{CO}_{2}$ reduction over $\mathrm{Cu}(100)$ at different flow rates of $\mathrm{CO}_{2}$. Under mass transport limited conditions, increased reactant availability will affect the product distribution; thus, this was used to identify under what potentials the reaction is governed by the intrinsic kinetics. Reprinted from Resasco et al. ${ }^{209}$ Copyright (C) 2017, American Chemical Society.

Potential-dependent trends can be difficult to discuss in the context of the $\mathrm{pH}$ effect. First of all, it is important to realize that when the $\mathrm{pH}$ at the electrode surface significantly deviates from that in the bulk if the potential is compared to the (bulk) RHE equilibrium potential without accounting for local $\mathrm{pH}$ gradients, then the catalytic overpotential is overestimated, which can cause errors in mechanistic interpretations. ${ }^{110,150,206}$ This has led some researchers to report results on a "corrected RHE" scale that uses the local $\mathrm{pH}$ as estimated from mathematical modeling. ${ }^{218}$ This has also motivated experimental measurement of the $\mathrm{pH}$ at the electrode/electrolyte interface under $\mathrm{CO}_{2} \mathrm{R}$ conditions. While this has remained a difficult challenge, there are two recent reports that have attempted to do so using attenuated total reflectance surface-enhanced infrared absorption spectroscopy (ATR-SEIRAS) to measure \& correlate the relative intensities of species involved in the buffer equilibria reactions with the local $\mathrm{pH}$ near the electrode surface. ${ }^{149,150}$ There are a few discrepancies between these studies, as this is an active area of method development, and more work should be done to validate and further advance these techniques. 
Secondly, as discussed in Section 2.2, it is also important to keep in mind that the extent of $\mathrm{OH}^{-}$ concentration polarization and thus local $\mathrm{pH}$ at a given applied potential will differ between catalysts and electrochemical cells. Catalysts with high surface areas will be more susceptible to $\mathrm{OH}^{-}$ concentration polarization at lower overpotentials. On the plus side, this likely explains the $\mathrm{CH}_{4}$ suppression \& enhanced selectivity to $\mathrm{C}_{2+}$ products typically observed on high surface area catalysts, which will be discussed in more detail in Chapter 6. This can also lead to HER suppression by local depletion of buffer species that act as competent proton donors, ${ }^{222,224}$ as discussed in Subsection 3.3.3. Unfortunately this also causes difficulties comparing potential-dependent $\mathrm{CO}_{2} \mathrm{R}$ performance between catalysts with very different roughness factors. This is not only an issue for high surface area catalysts though; even planar catalysts can exhibit different activity and selectivity if evaluated in electrochemical cells with different hydrodynamic boundary layer thicknesses. ${ }^{101}$ Therefore, it is important to keep all of these factors in mind when drawing conclusions about potential-dependent activity and selectivity.

Finally, it is worth noting that a gas diffusion electrode (GDE), while not suited for fundamental studies due to a complex interface, can in principle combine the best of both worlds, enabling both high $\mathrm{pH}$ conditions and sufficient mass transport of $\mathrm{CO}_{2}$ even with high surface area catalysts. See Section 6.3 for further discussion of these vapor-fed systems.

\subsubsection{Cation Effects}

It has been well documented in the literature that electrochemical $\mathrm{CO}_{2} \mathrm{R}$ activity \& selectivity is influenced by the alkali metal cation in the electrolyte. Initial work by Hori and Murata showed that the selectivity of $\mathrm{CO}_{2}$ and $\mathrm{CO}$ reduction on polycrystalline $\mathrm{Cu}$ was strongly influenced by cation size, with larger cations increasing the selectivity towards formation of $\mathrm{C}_{2+}$ species and decreasing the selectivity for the HER. ${ }^{225}$ Since then, a number of reports have confirmed and expanded on these experimental observations. ${ }^{98,209,215,226,227}$ While the experimental trends are largely in agreement, there has been some disagreement over the explanation for this effect. Hori and Murata rationalized the change in selectivity with cation size by the difference of outer Helmholtz plane (OHP) potential, based 
on Frumkin's theory, ${ }^{228}$ whereby the OHP potential will shift to more positive values with the degree of specific adsorption, which increases with cation size due to their lower hydration numbers. ${ }^{225}$ Recent theoretical work, however, has suggested that specific adsorption of alkali cations is not applicable to the conditions of $\mathrm{CO}_{2} \mathrm{R},{ }^{215}$ in part due to the very negative reduction potentials of alkali ions calculated on transition metal electrodes. ${ }^{229}$ In addition, oxygen reduction studies have shown that alkali cations primarily interact non-covalently, with surface X-ray scattering data suggesting that the cations are not adsorbed and remain partially solvated at the interface. ${ }^{230,231}$ DFT calculations demonstrate that solvation increases the favorability of specific adsorption and suggest a low coverage of partially hydrated $\mathrm{K}^{+}$is thermodynamically favorable under high cathodic potentials and $\mathrm{pH}$ conditions. ${ }^{232}$ Due to the complex nature of the electrochemical interface, it is difficult to say with certainty to what extent alkali cations interact with the electrode surface. Experimental identification of the mechanism(s) by which cations affect surface electrochemical processes (i.e., via specific adsorption and/or non-specific interactions) is extremely challenging and remains an open question. We will not distinguish that here and instead simply focus on the potential impacts that cations on/near the surface could have on $\mathrm{CO}_{2} \mathrm{R}$.

Electrochemical $\mathrm{CO}_{2}$ reduction potentials are typically more negative than the potential of zero charge (PZC) on low-index facets of $\mathrm{Cu}(\sim 0.7 \mathrm{~V} v s$. SHE$),{ }^{191}$ so solvated cations should accumulate on/near the surface of the electrode during reaction. This can block active sites on the surface, alter the binding energy or coverage of reaction intermediates, and/or influence the barriers of elementary steps. ${ }^{232}$ The presence of the cation near the metal surface also gives rise to high electric fields of roughly $-1 \mathrm{~V} / \AA$ in the vicinity of the ion (within $5 \AA$ ). ${ }^{233,234}$ While cations in the bulk of the electrolyte are stable to hydrolysis, electrostatic interactions between the solvated cation and cathode lower the pKa in the vicinity of the electrode; the magnitude of this decrease in pKa increases with larger alkali cation size and larger cathodic potentials. ${ }^{215}$ Thus, it was proposed that this allows larger cations to act as a $\mathrm{pH}$ buffer, offsetting the increase in local $\mathrm{pH}$ induced by concentration polarization and restoring the local $\mathrm{CO}_{2}$ concentration closer to the bulk value. ${ }^{215}$ This theoretical model matched their experimental measurements, but it must be noted that the experiments were performed under strong mass transport limitations. Thus, this model does not explain that fact that increasing activity and selectivity to $\mathrm{C}_{2+}$ 
products are observed both at lower overpotentials when not in a concentration polarized regime, as well as during COR, which does not have the same dependence of reactant concentration on $\mathrm{pH} .{ }^{209,227}$

Two recent studies have instead suggested that the primary role of the cation is to act as a promoter, stabilizing certain $\mathrm{CO}_{2} \mathrm{R} / \mathrm{COR}$ intermediates on the surface that have favorable electrostatic interactions with solvated cations. ${ }^{209,227}$ Specifically, cation-created electrostatic field stabilization decreases the energy for $* \mathrm{CO}_{2}$ adsorption, the precursor to $2 \mathrm{e}^{-}$products, and $\mathrm{C}-\mathrm{C}$ coupling to form *OCCO or $* \mathrm{OCCHO}$, possible precursors to $\mathrm{C}_{2+}$ products. ${ }^{209}$ This explains the trends observed not only for $\mathrm{Cu}$, but is also consistent with $\mathrm{CO}_{2} \mathrm{R}$ selectivity enhancements to $2 \mathrm{e}^{-}$products observed on $\mathrm{Ag}^{209,215,235}$ and Sn. ${ }^{209}$ This alone, however, does not explain the observed trend with cation size. For that, DFT calculations by Resasco et al. revealed that larger hydrated cations are more energetically favored at the outer Helmholtz plane than smaller ones, which suggests that a higher concentration of cations will accumulate with increasing cation size leading to a larger local electric field. ${ }^{209}$ The same researchers then provided support for this hypothesis by conducting experiments in mixed electrolytes, which showed that only a small percentage of the larger cations is needed to observe their influence on the intrinsic rates of formation of certain reaction products (e.g., $\leq 1$ order of magnitude increase in partial current densities for $\left.\mathrm{C}_{2} \mathrm{H}_{4}, \mathrm{C}_{2} \mathrm{H}_{5} \mathrm{OH}\right){ }^{209} \mathrm{~A}$ recent study using attenuated-total internal reflection Fourier-transform infrared (ATR-FTIR) spectroscopy has provided further evidence for this cation promotion effect, as it was found that with increasing cation size, surface-adsorbed CO molecules experience a greater interfacial electrostatic field that accelerates its reduction kinetics and acts to shift the $\mathrm{C} \equiv \mathrm{O}$ stretch frequency to lower energies. ${ }^{138}$

Future work in this area could expand on the findings for these monovalent alkali cations by investigating the impacts of divalent or trivalent cations. For example, $\mathrm{CO}_{2} \mathrm{R}$ rate increases have been observed over a $\mathrm{Cu}-\mathrm{Sn}-\mathrm{Pb}$ alloy cathode as the size and surface charge of the cation in the supporting electrolyte increases from $\mathrm{Na}^{+}$to $\mathrm{La}^{3+} .{ }^{236}$ There has also been some interest in the use of ionic liquids for $\mathrm{CO}_{2} \mathrm{R}$ after initial reports showed increased selectivity to $\mathrm{CO}$ at low overpotentials on $\mathrm{Ag}, \mathrm{MoS}_{2}$ and Pt electrodes. ${ }^{237-239}$ It has been hypothesized that the presence of ionic liquids can stabilize reaction intermediates, help bind the $\mathrm{CO}_{2}$ molecule, and/or suppress the HER by blocking active sites on the 
surface. While this could be a promising avenue to explore, we offer a word of caution, as it has also been found that the ionic liquids break down during $\mathrm{CO}_{2} \mathrm{R},{ }^{240,241}$ which can cause difficulties with product detection and limit its practicality for (re)use at scale. Finally, in moving towards practical $\mathrm{CO}_{2} / \mathrm{CO}$ electrolyzers, which will likely be vapor-fed reactors that employ GDEs, we will need to carefully consider how to engineer the ionomer/membrane moieties to promote stabilization of intermediates in a similar manner to the cation effects discussed here.

\subsubsection{Anion Effects}

The vast majority of $\mathrm{CO}_{2}$ reduction catalysis has been studied in $\mathrm{KHCO}_{3}$ electrolytes due to the $\mathrm{CO}_{2}$-(bi)carbonate-water equilibrium, which helps maintain a neutral bulk $\mathrm{pH}$. As a result, the impacts of the electrolyte anion have been studied to a lesser extent. Hori and coworkers presented results for constant current electrolysis in various electrolyte solutions, which showed that hydrocarbons and alcohols are the preferred products in $\mathrm{KCl}, \mathrm{KClO}_{4}, \mathrm{~K}_{2} \mathrm{SO}_{4}$, and dilute $\mathrm{KHCO}_{3}$ solutions, while $\mathrm{H}_{2}$ formation dominates in $\mathrm{K}_{2} \mathrm{HPO}_{4}$ solutions. ${ }^{84,86}$ These findings were explained in terms of a nonequilibrium local region of high $\mathrm{pH}$ close to the electrode. As discussed earlier, $\mathrm{OH}^{-}$ions are released during electroreduction, which can be neutralized by $\mathrm{HCO}_{3}^{-}$or $\mathrm{H}_{2} \mathrm{PO}_{4}^{-}$. Thus, in the non-buffering electrolytes or in dilute $\mathrm{KHCO}_{3}$, there is likely insufficient neutralization leading to a rise in $\mathrm{pH}$, which favors $\mathrm{CO}_{2} \mathrm{R}$ over $\mathrm{HER}$ and $\mathrm{C}_{2+}$ over $\mathrm{C}_{1}$ products. ${ }^{84,86}$ Other studies on the impact of $\mathrm{KHCO}_{3}$ concentration have mirrored these trends, showing that with increasing anion concentration the rates of hydrogen evolution and methane production increase, again pointing to local $\mathrm{pH}$ effects as the cause. ${ }^{110,242}$

A recent report, however, found that the changes in $\mathrm{pH}$ near the electrode surface were insufficient to explain differences in activity and selectivity observed with changes in anion buffering capacity; instead, it was proposed that these differences result from the ability of buffering anions to donate hydrogen directly to the electrode surface. ${ }^{218}$ While water is expected to be the proton source under $\mathrm{CO}_{2} \mathrm{R}$ conditions, it is possible that buffering anions can serve as proton donors given the lower pKa values compared to water. Thus, products where the rate-limiting step involves a proton transfer 
$\left(\mathrm{H}_{2}\right.$ and $\left.\mathrm{CH}_{4}\right)$ will be impacted by changes in the hydrogen source. Accordingly, it was found that $\mathrm{H}_{2}$ and $\mathrm{CH}_{4}$ activity increased ( $\leq 1$ order of magnitude) with decreasing $\mathrm{pKa}$ of the buffering anion and were not impacted by non-buffering anions, while formation of $\mathrm{CO}, \mathrm{HCOO}^{-}$, and $\mathrm{C}_{2+}$ products were insensitive to anion identity. ${ }^{218}$ This suggests that the buffering anions can serve as competent proton donors, with their effectiveness increasing with decreasing pKa. The impact was less pronounced once the local $\mathrm{pH}$ (as determined by mathematical modeling) was taken into account by plotting the activities on a calculated RHE scale; future studies should seek to test these effects over a wider range of potentials in case transport effects were also playing a role. Interestingly, this could still shed some light on one mechanism by which $\mathrm{H}_{2}$ evolution may be suppressed during $\mathrm{CO}_{2} \mathrm{R}$; depletion of $\mathrm{HCO}_{3}{ }^{-}$under basic conditions could impose mass transport limitations for the HER and limit the ability for this buffer to serve as a proton donor. ${ }^{222,224}$ Especially for mesoporous electrodes with high RFs and inhibited diffusion, this could serve to slow the rate of HER until sufficient overpotential to drive the sluggish water reduction kinetics is achieved.

In addition to bicarbonate serving as a $\mathrm{pH}$ buffer and proton source, recent studies have also discussed the possibility of bicarbonate serving as a $\mathrm{CO}_{2}$ source. ${ }^{119,139,140}$ Using a combination of in-situ spectroscopic analysis (ATR-SEIRAS) and isotopic labeling, along with mass spectroscopy (MS) or potential stepping techniques, two reports proposed that the $\mathrm{CO}_{2}$ source during $\mathrm{CO}_{2} \mathrm{R}$ is $\mathrm{CO}_{2}$ in equilibrium with bicarbonate anions instead of free $\mathrm{CO}_{2}$ molecules supplied to the solution. ${ }^{119,140}$ This hypothesis, however, relies on the critical assumption of fast equilibrium between $\mathrm{CO}_{2}$ and bicarbonate, which may not be the case since rate constants for the other equilibrium reactions are several orders of magnitude larger. ${ }^{139}$ With the increasing trend of in-situ spectroscopic analysis during $\mathrm{CO}_{2} \mathrm{R}$, the role of bicarbonate is an active area of research; thus, continued work should be done to further elucidate the $\mathrm{CO}_{2} \mathrm{R}$ reaction mechanisms in bicarbonate electrolytes.

Finally, it is also worth noting that there have been various studies on the influence of halide ions on $\mathrm{Cu}$ electrochemistry. ${ }^{95,96,117,243-250}$ Some reports suggest that there will be specific adsorption of these anions on the $\mathrm{Cu}$ surface, which could alter the charge density and selectivity for $\mathrm{CO}_{2} \mathrm{R}$ products, as well as aid in suppression of the HER. ${ }^{244-249}$ However, many studies show that the presence of halide 
ions primarily leads to changes in the $\mathrm{Cu}$ surface structure \& morphology; in particular, oxidationreduction cycles in the presence of halide ions has been one strategy used to increase selectivity to $\mathrm{C}_{2+}$ products, such as $\mathrm{C}_{2} \mathrm{H}_{4}$ (see Chapter 6 for further discussion). ${ }^{95,96,117,243,244,249,250}$ Thus, it is currently unclear what the dominating effect(s) of employing halides are, so this represents another area for future studies aimed at better understanding ionic interactions during $\mathrm{CO}_{2} \mathrm{R}$ catalysis.

\subsection{Electrochemical Conditions}

In addition to electrolyte effects, several studies outline the importance of the electrochemical cell design, components and operating conditions. ${ }^{88,97,251}$ While an in-depth discussion of these topics is outside the scope of this review, there are a few considerations and trends that we will touch upon here. Most $\mathrm{CO}_{2} \mathrm{R}$ studies to date have been conducted under ambient temperature and pressure, but these factors have also been explored to manipulate the local reaction conditions. Here we will briefly discuss some of the trends observed under non-ambient environments.

\subsubsection{Temperature \& Pressure}

From Henry's Law and the acid/base buffer equilibria $\left(\mathrm{CO}_{2} / \mathrm{HCO}_{3}{ }^{-} / \mathrm{CO}_{3}{ }^{-2}\right)$, it is understood that increasing the concentration of dissolved $\mathrm{CO}_{2}$ in the electrolyte can be achieved by decreasing temperature or increasing pressure (Figure 12)..$^{2,102,103,110,252-255}$ As a result, several studies have looked at varying the temperature $83,102,104,162,252-255$ and pressure ${ }^{102,103,110,201,226}$ during $\mathrm{CO}_{2} \mathrm{R} / \mathrm{COR}$. In general, it is difficult to draw direct comparisons between these limited studies due to the use of different electrolytes, applied voltages, techniques (galvanostatic vs. potentiostatic) and temperature/pressure ranges. However, a few high level trends can be extracted from their findings. Across the board, decreasing temperature was found to increase selectivity to $\mathrm{CH}_{4} \cdot{ }^{83,102,104,162,252-255}$ Trends for the other products are not as clear, although some results do show an increased production rate of $\mathrm{C}_{2} \mathrm{H}_{4}{ }^{83,104}$ and/or $\mathrm{CO}^{83,255}$ at higher temperatures. The rate of $\mathrm{H}_{2}$ evolution was found to be suppressed at lower temperatures and greatly enhanced at higher temperatures. ${ }^{83,102,252,253,255}$ The overall rate of $\mathrm{CO}_{2} \mathrm{R}$ was 
impacted by temperature to a lesser degree, ${ }^{102}$ with some studies finding minimal changes in the total partial current density for $\mathrm{CO}_{2} \mathrm{R}$ below room temperature ${ }^{4,104,105}$ and modest increases in reaction rate above room temperature. ${ }^{104,255}$ This leads to overall higher selectivity for $\mathrm{CO}_{2} \mathrm{R}$ at lower temperatures, and lower selectivity at higher temperatures where the HER becomes dominant. The reasons for these temperature effects are not entirely clear; changes in temperature affect the $\mathrm{CO}_{2}$ solubility, thermal diffusion rates, $\mathrm{pH}$, solution resistance, and may also alter adsorption equilibria. More detailed studies are needed to better understand the impact of temperature on $\mathrm{CO}_{2} \mathrm{R}$ product selectivity over a broader range of potentials.

As $\mathrm{CO}_{2}$ or $\mathrm{CO}$ pressure decreases below $1 \mathrm{~atm}$, the HER is dominant and minimal $\mathrm{CO}_{2} \mathrm{R} / \mathrm{COR}$ is detected. ${ }^{201,226}$ Meanwhile, with increased pressures, the total $\mathrm{CO}_{2} \mathrm{R}$ current generally increases (with the exception of the highest pressures in Hara et al.,${ }^{103}$ where there is significant missing Faradaic efficiency in their galvanostatic experiments at 40 and $60 \mathrm{~atm}) .{ }^{102,103,110}$ These trends are likely due in large part to the changes in dissolved concentration of $\mathrm{CO}_{2}$ with pressure. Interestingly, one study found that $\mathrm{C}_{2} \mathrm{H}_{4}$ selectivity increased with increased pressure, which was attributed to an increase in local $\mathrm{CO}$ concentration and surface coverage. ${ }^{110}$ Again, more systematic studies are needed to better understand the impact of pressure over a broader range of potentials and conditions. While operation of $\mathrm{CO}_{2} \mathrm{R}$ under ambient temperature and pressure has been discussed as a potential advantage of electrochemical $\mathrm{CO}_{2}$ recycling compared to other processes, we should not limit the scope of study to ambient conditions. Altering temperature and pressure remain areas ripe for further investigation, both to gain increased mechanistic understanding of electrochemical vs. chemical steps and for identifying operating conditions that achieve optimal activity and selectivity for $\mathrm{CO}_{2} \mathrm{R}$ to desired product(s). 


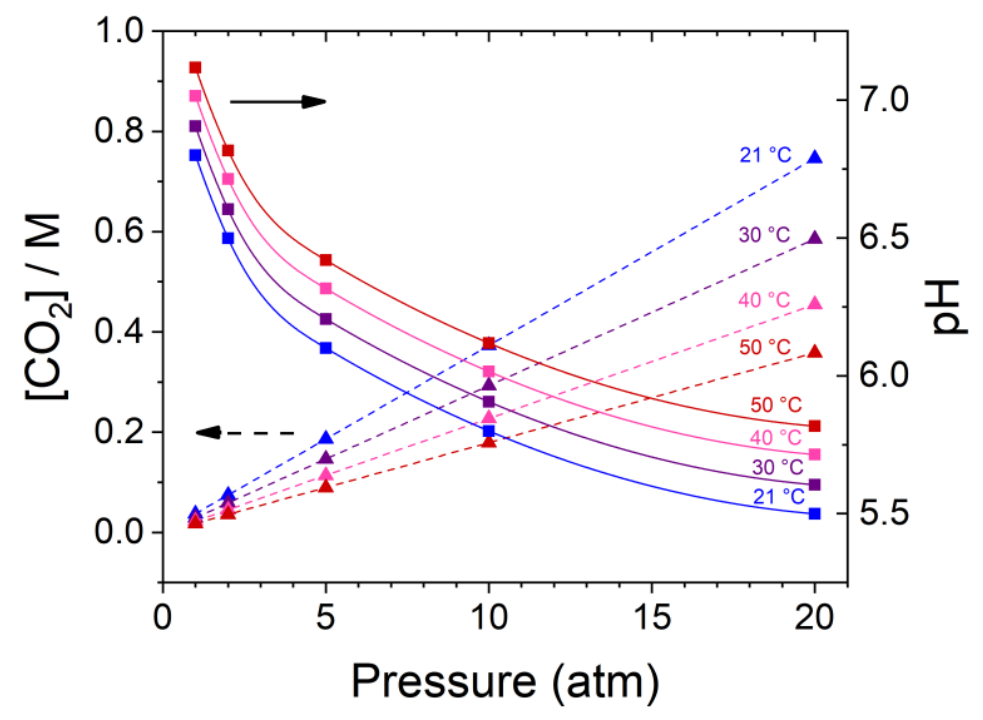

Figure 12: Effect of pressure and temperature on the concentration of dissolved $\mathrm{CO}_{2}$ in the electrolyte, $\left[\mathrm{CO}_{2}\right]$, and $\mathrm{pH}$. Adapted from T. Hatsukade. ${ }^{256}$ This work is licensed under a Creative Commons Attribution Noncommercial 3.0 United States License (http://creativecommons.org/licenses/bync/3.0/us/).

\subsection{Summary}

Electrochemical $\mathrm{CO}_{2}$ reduction on copper is a complex reaction involving multiple steps, intermediates, and reaction pathways. It is also highly sensitive to many factors from the atomic scale to the reactor scale including the electrode surface structure and morphology, electrolyte $\mathrm{pH}$ and identity/quantity of ions present, degree of mass transport, temperature, pressure, etc.

Single crystals and oriented surfaces represent useful tools to study the impact of crystal facet on $\mathrm{CO}_{2} \mathrm{R}$ performance of $\mathrm{Cu}$ electrodes. In particular, interesting trends in product distribution have been reported as a function of surface structure. Among the planar surfaces, $\mathrm{Cu}(100)$ was shown to yield a particularly high ethylene/methane ratio, and the selectivity toward ethylene could be further improved by adding steps to the surface. ${ }^{152-154,159}$ These findings can help provide design principles to steer the selectivity of catalyst materials towards important products. On the other hand, no significant trends in activity have been reported. This could be due to defects or step sites existing on the surface and/or formed in-situ, leading to similar overall activity on all the $\mathrm{Cu}$ single crystal surfaces investigated. We would like to emphasize the need for more quantitative data from oriented surfaces, 
preferably of single crystal quality, as a function of potential. This is vital in order to obtain further mechanistic information, which can lead to identification of key parameters that can be optimized to steer selectivity and activity toward desired products.

At the same time, it is also important to keep in mind that the crystallographic orientation of the surface of single crystal electrodes can differ from their bulk orientation. Some reports indicated that the surface structure of the electrodes was strongly dependent on the choice of pre-treatment procedure, ${ }^{153,172}$ while others showed that certain electrodes tended to restructure under reaction conditions. ${ }^{173,174,179-182}$ While the causes for restructuring require further investigation, some likely reasons include effects from the presence of $\mathrm{CO}$, the negative applied potentials, or other factors from the local reaction environment, such as electrolyte effects. We emphasize that phenomena exhibited by crystals or films oriented in a specific direction may not originate from the bulk orientation, but rather from the defects present at the surface, as illustrated in Figure 7; as such, scientists should be cautious in making direct comparisons between the results from experiments on single crystals to DFT-based models. In addition, due to the complications we discussed above, we strongly recommend that future studies of single crystals and other oriented systems investigate the structural properties the electrodes in-situloperando whenever possible.

The electrolyte presents several variables that can be used to tune $\mathrm{CO}_{2} \mathrm{R}$ activity and selectivity. In particular, the impact of $\mathrm{pH}$ is a complex subject that causes several competing effects on the local reaction environment. A high interfacial $\mathrm{pH}$ is beneficial for increasing selectivity towards $\mathrm{CO}_{2} \mathrm{R}$ and reducing the overpotential required to form desirable $\mathrm{C}_{2+}$ products on an RHE scale; it becomes a detriment, however, when there is insufficient mass transport of $\mathrm{CO}_{2}$, and the acid-base equilibria between $\mathrm{CO}_{2}, \mathrm{HCO}_{3}{ }^{-}, \mathrm{CO}_{3}{ }^{2-}$ and $\mathrm{OH}^{-}$causes a significant decrease in the local $\mathrm{CO}_{2}$ concentration. The extent of concentration polarization is determined by the overall flux of species both to and from the electrode surface. This depends not only on the degree of mixing within an electrochemical cell, which impacts the hydrodynamic boundary layer thickness, but also the overall reaction rate (current density), which is impacted by the surface area of the catalyst. We strongly recommend that conclusions about intrinsic activity and selectivity only be drawn from data that is not significantly impacted by mass transport effects. 
The electrolyte cation and anion also play important roles in the catalysis. Experimental trends for $\mathrm{CO}_{2} \mathrm{R}$ on $\mathrm{Cu}$ have consistently demonstrated increased activity and selectivity for $\mathrm{C}_{2+}$ products with increasing alkali cation size, although the hypotheses behind these effects have evolved over time. 98,209,215,225-227 By combining theoretical and experimental methods, greater mechanistic insights have been obtained. While the extent and mechanism of alkali cation interaction with the surface is still an open question, the localized electrostatic fields they create is proving to be a critical factor impacting $\mathrm{CO}_{2} \mathrm{R}$ activity \& selectivity. Indeed, DFT calculations reveal that cation-created field effects are crucial for $\mathrm{CO}_{2}$ activation on weak binding surfaces ${ }^{233}$ and $\mathrm{CO}$ dimerization on $\mathrm{Cu}$ at room temperature..$^{234}$ Anion effects have often been discussed in relation to the local $\mathrm{pH}$ at the electrode surface, ${ }^{84,86,110,242}$ but it also been proposed that buffering anions can donate hydrogen directly to the electrode surface, which increases the rates of $\mathrm{H}_{2}$ and $\mathrm{CH}_{4}$ formation. ${ }^{218}$ Recent studies have additionally discussed the possibility of bicarbonate serving as a $\mathrm{CO}_{2}$ source, but further work is needed to determine the extent to which this may occur. Finally, temperature and pressure represent additional levers by which to tune the catalysis since the concentration of $\mathrm{CO}_{2}$ in the electrolyte can be increased via increasing pressure or decreasing temperature; in addition, these electrochemical conditions may also alter adsorption equilibria and diffusion rates. While some trends in product selectivity have been observed, this remains an area in need of further systematic investigations.

In summary, in this Chapter we discussed how a variety of different experimental factors can impact $\mathrm{CO}_{2} \mathrm{R}$ on $\mathrm{Cu}$, the mechanistic insights that have been gained from studying them, and how they can be tailored to optimize activity and selectivity to desired product(s), including areas for future study. In the next Chapter we will build upon these experimental findings and take a more in depth look at the complementary theoretical descriptions of $\mathrm{CO}_{2} \mathrm{R}$ on $\mathrm{Cu}$ and how these methods have helped shape our mechanistic understanding of the factors governing $\mathrm{CO}_{2} \mathrm{R}$ activity and selectivity.

\section{Theoretical Studies on Copper}

In recent years, developments in ab initio methods for the simulation of surface and 
electrochemical reactions have enabled theoretical mechanistic investigations and theory-guided catalyst design. ${ }^{58}$ Electrochemical $\mathrm{CO}_{2}$ reduction is particularly challenging due to the complex reaction networks and the importance of activation energies and cation effects. ${ }^{169,233}$ In what follows, we provide an introduction to state-of-the-art modeling approaches for electrochemical processes and discuss the major conclusions, controversies, and open challenges in recent work.

\subsection{Introduction to Theoretical Approaches}

Modeling electrochemical interfaces from an atomistic perspective presents many open challenges. In general, the thermodynamics for electrochemical reactions are relatively simple to obtain with the computational hydrogen electrode model, ${ }^{257}$ which relies on calculations of adsorption energies and a $\mathrm{H}_{2}$ gas reference to account for the free energy of proton-electron pairs, i.e. $\mu\left(\mathrm{H}^{+}\right)+\mu(\mathrm{e})=$ $\frac{1}{2} \mu\left(\mathrm{H}_{2}\right)-\mathrm{e} U$, where $U$ is the potential vs. the reversible hydrogen electrode (RHE). For a protonelectron transfer to ${ }^{*} \mathrm{CO}$ to form ${ }^{*} \mathrm{CHO}$, for instance, the corresponding free energy change is

$$
\Delta G=\mu\left(\mathrm{CHO}^{*}\right)-\mu\left(\mathrm{CO}^{*}\right)-\left[\frac{1}{2} \mu\left(H_{2}\right)-e U\right]+\Delta G_{\text {solv }}+\Delta G_{\text {field }}
$$

where $\Delta G_{\text {solv }}$ and $\Delta G_{\text {field }}$ refer to contributions of solvation and field. ${ }^{258,259}$ For a given reaction step, a thermodynamic limiting potential $U_{\mathrm{L}}$ can be determined, which is defined as the potential at which a reaction step becomes exergonic, where $\Delta G=0$. For a reaction pathway, the corresponding limiting potential is given by that of the step with the highest reaction free energy. This approach has been applied to a mechanistic analysis of $\mathrm{CO}_{2} \mathrm{R}$ pathways towards $\mathrm{C}_{1}$ products on stepped $\mathrm{Cu}(211)$, and $U_{\mathrm{L}}$ for various products have been shown to be correlated with experimental onset potentials on polycrystalline copper. ${ }^{202}$ Likewise, the onset potentials for $\mathrm{CH}_{4}$ or $\mathrm{CH}_{3} \mathrm{OH}$ across polycrystalline transition metals have been shown to follow trends in the corresponding $U_{\mathrm{L}}{ }^{58}$

While a thermodynamic analysis using the limiting potential $U_{L}$ as a key metric has shown mechanistic insight and correlation with experimental data on transition metals, this presents only a necessary but insufficient criterion for activity. Determination of activation energies allows for turnover frequencies to be evaluated as a function of potential. Surface hydrogenation barriers (i.e. Langmuir- 
Hinshelwood type) through an adsorbed $\mathrm{H}^{*}$, where electron transfer has already occurred, are easy to determine in vacuum, but electrochemical barriers (sometimes referred to as Eley-Rideal260 type) are considerably more challenging. In addition, as discussed in Section 3.3, ion and solvent effects have been shown to be important factors determining activity and selectivity towards various products. The investigation of electrochemical activation energies and the resultant kinetics and selectivities require models that explicitly account for the potential, solvent, and ions, where much method development is ongoing and where considerable variations exist in the energetics for similar processes determined using different approaches. In recent years, $\mathrm{CO}_{2} \mathrm{R}$ energetics have been studied theoretically using implicit solvent models that include a continuum description of the ions, ${ }^{261,262}$ explicit solvent models where water and ions are considered atomistically, ${ }^{169,260}$ and also a H-shuttling approach ${ }^{262-264}$ where a water molecule shuttles protons from the surface and back. We will first briefly summarize these different theoretical approaches.

\subsubsection{Implicit Models}

In general, the inclusion of solvent molecules and ions adds many extra degrees of freedom and computational time relative to vacuum|metal calculations for activation barrier calculations. Implicit solvent models apply a continuum model of the electrolyte, which corresponds to a negligible increase in computational time. The charge at the interface can be varied continuously, such that the potential is easily tuned. While simple and computationally cheap, these models present several challenges. One of the most widely used implementations of the implicit method makes use of a simple linearized PoissonBoltzmann distribution of the ions, ${ }^{265}$ which does not account for effects of finite ion size and has been suggested in classical models to lead to unphysical interfacial capacitances. ${ }^{266}$ The potential of zero charge (PZC) has been proposed as a benchmark for these methods ${ }^{261,267}$ however, since PZCs and metal work functions are correlated, ${ }^{266}$ the agreement between experimental and theoretical PZC may reflect the accuracy of generalized gradient approximation (GGA) functionals in the determination of metal work functions more than the description of the solvent. Such a benchmark also does not evaluate the accuracy of the reaction energetics, where solvation or cation interactions can have a significant 
effect. Finally, a fundamental challenge associated with implicit solvent simulations at constant potential is that the ions are modeled to adiabatically follow the shifts in dipoles associated with a reaction event. These two processes, however, occur on very different timescales. ${ }^{268,269}$ The effect of such an assumption on the energetics has not been evaluated against explicit models.

\subsubsection{Explicit Models}

Explicit solvent methods allow for an atomistic level picture of solvation and cation effects, but at the expense of significantly increased computational cost and the introduction of other challenges. Since ions are modeled explicitly and within finite-sized unit cells, the potential is not continuously variable. Proton-electron transfer processes modeled in finite-sized unit cells also give rise to significant shifts in the potential along the reaction pathway. These issues can be mitigated using a capacitor model of the interface, which allows for the extrapolation of calculated barriers to the limit of infinite cell sizes, corresponding to the constant-potential limit. ${ }^{270,271}$ State of the art GGA-level functionals predict a poor band alignment of solvent HOMO, LUMO with the metal Fermi level, which can give rise to artificial charge transfer across the interface and corresponding effects on the reaction energetics. ${ }^{272}$ Unrelated to band alignment is the partial charge of hydronium ions at the Helmholtz plane arising from overlap of solvent and metal charge, which affect the reaction energetics. ${ }^{273}$ Transition states have been determined using either nudged elastic band $(\mathrm{NEB})^{169,274}$ or metadynamics and potential of mean force ${ }^{260,275}$ the latter method includes entropic contributions from solvent reorganization, but at an increased computational cost.

\subsubsection{H-shuttling Model}

An alternative to full explicit solvent models has been put forth by Janik et al. ${ }^{263,264,276}$ In this approach, a water molecule is used to shuttle an adsorbed $\mathrm{H}^{*}$ from the surface to the adsorbate (Figure 13). This shuttling process is considered to provide an estimate of the associated proton-electron transfer barrier. The potential $v s$. RHE for the process is assumed to correspond to that where $\mathrm{H}^{*}$ in the initial state is equilibrated with a proton-electron pair, as determined using the computational hydrogen 
electrode. In contrast to explicit solvent calculations, potential shifts along the reaction pathway arising from shifts in charge are not considered. The transfer coefficient is assumed to be 0.5 plus a correction determined by shifts in the dipoles of the adsorbates. While the simplicity of this approach is compelling, the assumptions should be evaluated with more detailed models.

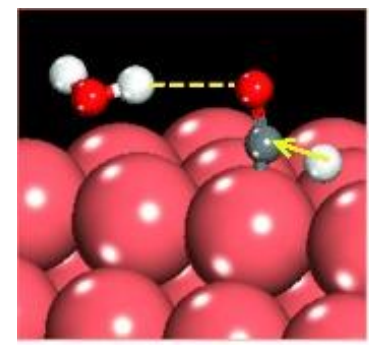

(a)

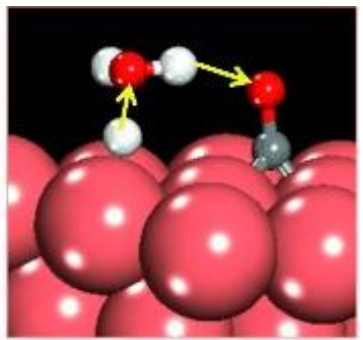

(b)

Figure 13: Water shuttling model for proton-electron transfer to $* \mathrm{CO}$ to form (a) ${ }^{*} \mathrm{CHO}$ and (b) $* \mathrm{COH}$. A single water molecule is included to shuttle an adsorbed $* \mathrm{H}$ and mimic proton-electron transfer from solution. It was found that $* \mathrm{CHO}$ formation occurs through direct transfer of $* \mathrm{H}$ to the $* \mathrm{CO}$. Reprinted with permission from Janik et al. ${ }^{264}$ Copyright (C) 2013, John Wiley and Sons.

\subsection{Theoretical Insights}

In general, thermodynamic analyses in the current literature have been performed using the computational hydrogen electrode model. The choice of functional, pseudopotentials, and the treatment of solvation should all affect the energies, but resultant trends and energies reported by different groups do not differ significantly (e.g. the similarity in trends in energetics of $\mathrm{Cu}(111)$ intermediates from Refs ${ }^{263,277}$ ). Theoretical electrochemical activation energies, on the other hand, widely differ with approach, due to the differences in the simplifying assumptions made in modeling the electrochemical interface.

Tables S2, S3, S4 summarize, respectively, the activation energies for key $\mathrm{CO}_{2} \mathrm{R}$ to $\mathrm{CO}$, initial $\mathrm{CO}$ reduction, and $\mathrm{C}-\mathrm{C}$ coupling steps determined using various methods, and, where available, their transfer coefficient $\beta$ and the barrier for the corresponding surface hydrogenation process. These tables can be found in Section 3 of the Supporting Information (SI). In this review, we assume the criterion for facile kinetics to be a turnover frequency of $1 / \mathrm{s}$ per site, which corresponds to approximately a 0.75 $\mathrm{eV}$ barrier at room temperature, ${ }^{278}$ and we have reported the barriers at $0 \mathrm{~V} v s$. RHE wherever possible. In reporting barriers determined with $\mathrm{H}$-shuttling, we considered the transfer of $\mathrm{H}^{*}$ through $\mathrm{H}_{2} \mathrm{O}$ to be classified as electrochemical (i.e., involving electron transfer) and a direct transfer of $\mathrm{H}^{*}$ as a surface 
hydrogenation. Overall, the differences in energetics and resultant mechanisms reported point to the importance of evaluating the assumptions made in the models of the electrochemical interface. In what follows, we discuss general insights and controversies in current theoretical investigations of $\mathrm{CO}_{2} \mathrm{R}$ on $\mathrm{Cu}$.

\subsubsection{Trends in Electrochemical Barriers}

One of the general insights from existing theoretical investigations is the trends in activation energies for proton-electron transfer towards different intermediates, which, given the complexity of $\mathrm{CO}_{2} \mathrm{R}$ pathways towards the many possible products (as shown later in Chapter 5, Figure 18), provides some qualitative guidance in the search for rate-determining steps. Using an explicit solvent model and NEB barrier calculations on various transition metals, proton-electron transfers to oxygen ends of reaction intermediates were found to be in general facile, while those to carbon or those involving $\mathrm{C}-\mathrm{O}$ bond scission are more difficult. ${ }^{274}$ Similarly, metadynamics ${ }^{260}$ with explicit solvent and $\mathrm{H}$ shuttling ${ }^{264,276}$ barrier calculations found that oxygen are hydrogenated primarily through electrochemical $v s$. direct $* \mathrm{H}$ transfer steps.

\subsubsection{CO as a Crucial Intermediate}

The pathway towards CO has been analyzed with several methods, as tabulated in Table S2. On $\mathrm{Cu}(111)$, both explicit solvent with NEB transition state search ${ }^{169}$ and $\mathrm{H}$-shuttling methods ${ }^{263,264}$ find the activation energies for $\mathrm{CO}_{2}$ reduction to ${ }^{*} \mathrm{CO}$ to require low overpotentials to reach the $0.75 \mathrm{eV}$ threshold for facile kinetics. On $\mathrm{Cu}(100), \mathrm{H}$-shuttling models ${ }^{276}$ report less surmountable barriers than explicit solvent with metadynamics, ${ }^{279}$ which give a net barrier of $0.8 \mathrm{eV}$ for $\mathrm{CO}_{2(\mathrm{~g})}$ to $* \mathrm{COOH}$ at -0.4 $\mathrm{V} v s$. RHE; the reason for this discrepancy may be differences in solvation or the simplifying assumptions behind the $\mathrm{H}$-shuttling model. Overall, a facile path towards $\mathrm{CO}$ is consistent with experimental reports that both $\mathrm{CO}$ and $\mathrm{CO}_{2}$ reduction give similar product distributions, suggesting $\mathrm{CO}$ to be a key reaction intermediate in $\mathrm{CO}_{2}$ reduction, as discussed earlier in Subsection 3.2.1. Formate, on the other hand, was shown to be a dead end, given that the free energy difference from ${ }^{*} \mathrm{OCHO}$ to 
${ }^{*} \mathrm{OCH}_{2} \mathrm{O}$ is $1.6 \mathrm{eV} .{ }^{202}$ This theoretical result is consistent with experiments using $\mathrm{HCOO}^{-}$as a feedstock, which gave no detectable products. ${ }^{86}$

\subsubsection{Pathway Towards Methane}

Using explicit water models, barriers determined using NEB and metadynamics suggest identical reaction intermediates from $\mathrm{CO}$ to $\mathrm{CH}_{4}$ on stepped $\mathrm{Cu}(211)^{169}$ and $\mathrm{Cu}(100)^{260}$ at $\mathrm{pH} 7$, respectively. The free energy diagram for $\mathrm{Cu}(211)$ is shown in Figure 14a, and the reaction species determined on $\mathrm{Cu}(100)$ are shown in Figure 14b. In this pathway, ${ }^{*} \mathrm{CO}$ to $* \mathrm{CHO}$ is the rate-determining step, which is followed by a series of proton-electron transfers to form ${ }^{*} \mathrm{CHOH},{ }^{*} \mathrm{CH},{ }^{*} \mathrm{CH}_{2},{ }^{*} \mathrm{CH}_{3}$, and $\mathrm{CH}_{4}$. In contrast to a purely thermochemical analysis, ${ }^{202}{ }^{*} \mathrm{CH}_{3} \mathrm{O}$ is not found to be an intermediate towards $\mathrm{CH}_{4}$ due to the insurmountable barrier for proton-electron transfer to ${ }^{*} \mathrm{CH}_{3} \mathrm{O}$ to form $\mathrm{CH}_{4}+$ *O. ${ }^{264,274}$ It must be noted that crucial differences exist between pathways determined by explicit and $\mathrm{H}$-shuttling models. Whereas explicit methods ${ }^{169,260}$ suggest that all steps following ${ }^{*} \mathrm{CO}$ are protonelectron transfers on $\mathrm{Cu}(211)$ and $\mathrm{Cu}(100),{ }^{\mathrm{a}} \mathrm{H}$-shuttling calculations suggest that ${ }^{*} \mathrm{CHO}$ is formed through a direct transfer of ${ }^{*} \mathrm{H}$ on both $\mathrm{Cu}(100)$ and $\mathrm{Cu}(111){ }^{263,280} \mathrm{On} \mathrm{Cu}(111), \mathrm{H}$-shuttling in conjunction with implicit solvent ${ }^{262}$ and explicit water ${ }^{169}$ methods suggest a ${ }^{*} \mathrm{COH}$ and ${ }^{*} \mathrm{CHO}$ intermediate, respectively.
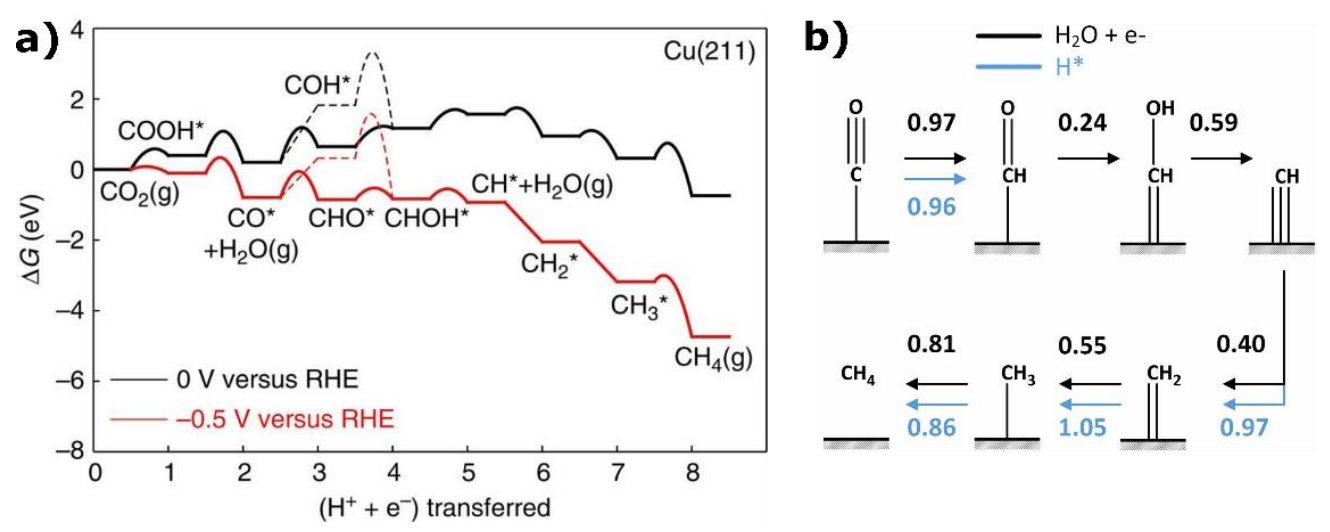

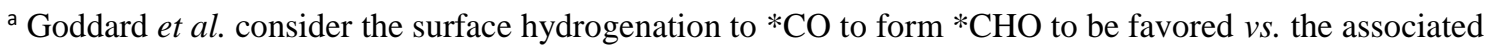
proton transfer, and report barriers of 0.96 and $0.97 \mathrm{eV}$ (at $-0.59 \mathrm{~V} v s$. RHE) respectively. However, the latter electrochemical barrier would go down substantially with decreasing potential, assuming a usual $\beta \sim 0.5$.
} 
Figure 14: a) Free energy diagram of $\mathrm{CO}_{2} \mathrm{R}$ to $\mathrm{CH}_{4}$ on $\mathrm{Cu}(211)$, model system for $\mathrm{Cu}$ steps. Reprinted from Liu et al. ${ }^{169}$ This work is licensed under a Creative Commons Attribution 4.0 International License (https://creativecommons.org/licenses/by/4.0/). b) Reaction intermediates towards $\mathrm{CH}_{4}$ determined on $\mathrm{Cu}(100)$. Reprinted from Cheng et al. ${ }^{260}$

\subsubsection{CO Dimerization}

Pathways toward $\mathrm{C}_{2+}$ products are considerably more complex and present more controversy amongst theoretical studies. Adding to this complexity is that certain $\mathrm{C}_{2+}$ intermediates with large dipole moments are dramatically affected by the fields induced by cations at the interface, which add additional degrees of freedom affecting the energetics (see Figure 15). Both vibrational spectra ${ }^{134,145-148}$ and kinetic models ${ }^{169,261}$ suggest $* \mathrm{CO}$ to be present on $\mathrm{Cu}$ under $\mathrm{CO}_{2}$ and $\mathrm{CO}$ reduction conditions, and the coupling of $2 * \mathrm{CO}$ to form a $* \mathrm{OCCO}$ dimer has been studied using several approaches. It was found that *OCCO on $\mathrm{Cu}(100),(111)$, and (211) have insurmountable dimerization barriers (>1 eV) in vacuum, but water solvation and cation effects together stabilize the $* \mathrm{OCCO}$ relative to $2 * \mathrm{CO}$ such that dimerization becomes facile..$^{234,281-283}$ Further, it was found that $\mathrm{Cu}(100)$ and stepped (211) exhibit lower barriers than $\mathrm{Cu}(111) .{ }^{283}$ Implicit solvent models with a continuum ion distribution should also provide a field-induced stabilization, though the charge and field distribution would not be as localized as in the case of an explicit cation; Head-Gordon et al. report a dimerization barrier of only $0.53 \mathrm{eV}$ at $0 \mathrm{~V} v s$. RHE on $\mathrm{Cu}(100)$ in the presence of a continuum distribution of charge in the solvent. ${ }^{261}$ Overall, the sensitivity of the energetics of this step to water solvation and cation-induced fields likely contributes to the tremendous variation in reported $\mathrm{CO}$ dimerization barriers on $\mathrm{Cu}$ facets: 0.53 to $1.3 \mathrm{eV}$ on $\mathrm{Cu}(100)$, and 0.89 to $1.7 \mathrm{eV}$ on $\mathrm{Cu}(111)$ (see Table $\mathbf{S 3}$ in SI). 


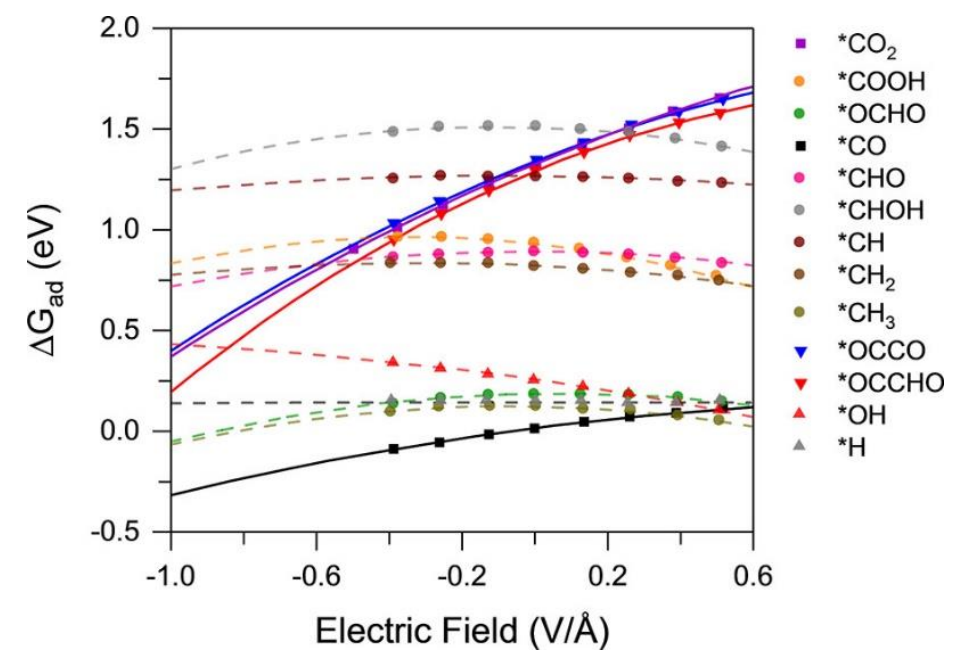

Figure 15: Field effects on various $\mathrm{CO}_{2} \mathrm{R}$ intermediates on $\mathrm{Cu}(111)$. The energy of each adsorbate as a function of field strength is obtained by applying a uniform electric field oriented perpendicular to the slab. Adsorbates that are most stabilized by field are $* \mathrm{CO}_{2}$, $* \mathrm{OCCO}, * \mathrm{OCCHO}$. Reprinted from Resasco et al. ${ }^{209}$ Copyright (C 2017, American Chemical Society.

\subsection{5 $\mathrm{C}_{2}$ Pathways}

Beyond $* \mathrm{OCCO}$, the determination of $\mathrm{C}_{2}$ mechanistic pathways and their facet dependence presents an open challenge, with different methods reporting different mechanisms. These differences point to the importance of method development and benchmarking efforts in $a b$ initio simulations of the electrochemical interface. Vacuum calculations on $\mathrm{Cu}(211)$ suggest that coupling barriers tend to decrease with increasing hydrogenation of the coupling species, ${ }^{281}$ and that the pathway to acetaldehyde and ethanol is thermodynamically downhill from ${ }^{*} \mathrm{OCCHO} .{ }^{91}$ With vacuum|metal calculations on $\mathrm{Cu}(100)$, a $* \mathrm{OCCOH}$ to $* \mathrm{OCC}$ pathway was found to be thermodynamically favored after $* \mathrm{CO}$ dimerization. ${ }^{284}$ Likewise, this pathway was found to be dominant in an ab initio, explicit solvent study on $\mathrm{Cu}(211)$, despite the difference in the treatment of the interface and in the facet. ${ }^{213}$ An analysis with the $\mathrm{H}$-shuttling model suggests that pure $\mathrm{Cu}(100)$ facets do not have facile barriers to form $\mathrm{C}_{2}$ products, and it is concluded that reconstruction of the surface is required for C-C coupling. ${ }^{276}$ However, these calculations were performed without cations and a full solvent layer, both of which can stabilize some crucial intermediates in the processes considered. An implicit solvent study on $\mathrm{Cu}(100)$ found $* \mathrm{OCCHO}$ to be a critical intermediate towards $\mathrm{C}_{2+}$ products, with $* \mathrm{OCHCHO}$ found to be the precursor to acetaldehyde and ethanol, and ${ }^{*} \mathrm{COCHOH}$ the precursor to ethylene. ${ }^{208}$ The former is in line with 
experimental observation that OCHCHO (glyoxal) can be reduced to acetaldehyde and ethanol. ${ }^{113,209}$ Finally, explicit solvent simulations with metadynamics on $\mathrm{Cu}(100)$ suggest that, at low overpotentials, $\mathrm{C}_{2+}$ products are formed through $\mathrm{CO}$ dimerization and subsequent proton-electron transfers towards ${ }^{*} \mathrm{HOCCOH}^{260}$ ( $v s$. the $*$ OCC intermediate proposed by Ref. ${ }^{284}$ ). At high overpotentials, it is assumed that adsorbed $* \mathrm{H}$ saturates the surface such that $* \mathrm{OCCHO}$ is formed through 2 Eley-Rideal steps of $\mathrm{CO}_{(\mathrm{g})}+* \mathrm{H} \rightarrow * \mathrm{CHO}$, then $* \mathrm{CHO}+\mathrm{CO}_{(\mathrm{g})} \rightarrow * \mathrm{OCCHO} .{ }^{260}$ We note, however, that the barrier reported for the first Eley-Rideal step is insurmountable at room temperature at $1.01 \mathrm{eV}$. Microkinetic models also suggest that at steady state the $* \mathrm{CO}$ coverage continues to dominate over $* \mathrm{H}$, even down to -1 $\mathrm{V},{ }^{169,261}$ and $* \mathrm{CO}$ has been observed with vibrational spectroscopy down to such potentials. ${ }^{147}$

\subsubsection{Kinetics}

Given the challenge of determining electrochemical activation energies, few mean-field kinetic models of CO reduction have been reported in the literature. ${ }^{169,213,261}$ Kinetics provide insight into rates, selectivities, intermediate coverages and competition amongst different pathways, all as a function of reaction conditions. However, since rates depend exponentially on energies (i.e. $r \sim \exp \left(-E_{d} / k T\right)$ ), large uncertainties are expected in predicted rates. For example, only a $0.1 \mathrm{eV}$ uncertainty in a barrier translates to over an order of magnitude uncertainty in rate at room temperature. Trends in general are therefore considered to be more reliable than absolute rates.

Recently, a kinetic model for $\mathrm{CO}$ reduction to $\mathrm{CH}_{4}$ on transition metals was developed. ${ }^{169}$ Based on the reaction pathway on $\mathrm{Cu}(211)$ (Figure 14a), it was assumed that the proton-electron transfer from ${ }^{*} \mathrm{CO}$ to ${ }^{*} \mathrm{CHO}$ is the limiting step for $\mathrm{CO}$ reduction on all transition metals. Scaling relations for the $\mathrm{H}-$ $\mathrm{CO}$ transition state and the corresponding kinetic activity volcano for $\mathrm{CO}$ reduction to $\mathrm{CH}_{4}$ were determined on (111) and (211) surfaces, which is shown in Figure 16. Adsorbate-adsorbate interactions, which come into play on metals that bind as strongly as $\mathrm{Cu}$ or more, were included with a first-order adsorbate interaction model. ${ }^{285}$ The stepped facet was found to be considerably more favorable for *CO protonation than flat ones, owing to the ease of $* \mathrm{CO}$ rotation and also the accessibility of the $\mathrm{C}$ end to proton transfer from the solvent. Stepped facets were therefore predicted to give orders of magnitude 
higher rates than flat ones. ${ }^{169}$ In fact, it is suggested that only a small fraction of stepped sites can account for the experimentally determined activity of polycrystalline transition metals ${ }^{204}$ which are overlaid in Figure 16a. ${ }^{169} \mathrm{Cu}$ already lies close to the peak of the activity volcano for materials that follow transition metal scaling, due to its intermediate ${ }^{*} \mathrm{CO}$ binding energy. Based on this analysis, two design criteria for active $\mathrm{CO}$ reduction catalysts were proposed: (i) stabilization of stepped sites and (ii) stabilization of the $* \mathrm{H}-\mathrm{CO}$ intermediates for a given $* \mathrm{CO}$ binding energy ${ }^{169}$ The corresponding contour plot of activity based on $* \mathrm{H}-\mathrm{CO}$ and $* \mathrm{CO}$ energies is given in Figure 16b. We note that the predicted significantly higher activity of steps has yet to be demonstrated experimentally, although studies of both single crystal ${ }^{1}$ and epitaxial films ${ }^{158}$ do suggest roughly an order of magnitude variation in activity amongst different facets. Step poisoning, undercoordinated surface defects, and/or surface reconstruction may contribute to this discrepancy between experimental and theoretical results, as discussed earlier in Section 3.1.
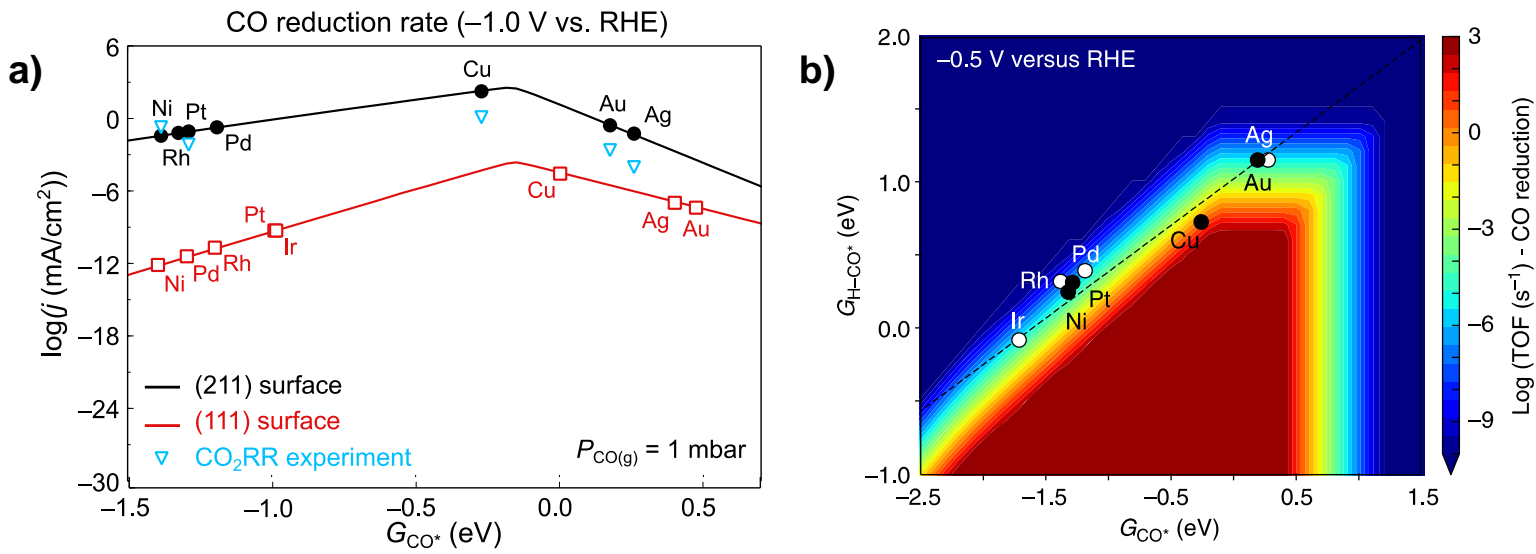

Figure 16: a) Kinetic volcano for $\mathrm{CO}$ reduction to $\mathrm{CH}_{4}$ on transition metals. $\mathrm{CO}_{2} \mathrm{R}$ Experiments from Kuhl et al. ${ }^{204} \mathrm{~A} 1 \mathrm{mbar} \mathrm{CO}$ pressure was assumed in the kinetic model to compare $\mathrm{CO}$ and $\mathrm{CO}_{2} \mathrm{R}$ rates. b) $2 \mathrm{D}$ Volcano reflecting the dependence of activity on $\mathrm{H}-\mathrm{CO}$ transition state and $\mathrm{CO}$ scaling. Both figures are reprinted from Liu et al. ${ }^{169}$ This work is licensed under a Creative Commons Attribution 4.0 International License (https://creativecommons.org/licenses/by/4.0/).

The $\mathrm{C}_{2}$ kinetic model of Head-Gordon et al. ${ }^{261}$ considers the initial C-C coupling steps, with energetics determined through an implicit solvent model. The coupling barriers for CO dimerization were found to slightly increase with more cathodic potential, such that the dimerization pathway shuts down in favor of CO-CHO coupling at high overpotential. The resultant kinetics suggested a decrease 
in $\mathrm{C}_{2+}$ products at moderate overpotentials, consistent with DEMS experiments on $\mathrm{Cu}(100) .{ }^{164}$ This picture is in contrast to an atomistic picture, where cation concentrations, and therefore $\mathrm{CO}$ dimerization rates, should increase with decreasing potential. The physical origin of these effects and the differences between conclusions obtained through implicit and explicit approaches requires further evaluation.

\subsubsection{Rate-Limiting Electron Transfers}

Rate-limiting electron transfers in $\mathrm{CO}_{2}$ reduction has been a recurrent and contentious theme in the current theoretical literature. In particular, $\mathrm{CO}$ dimerization has been postulated to have a ratelimiting electron transfer decoupled from proton transfer. ${ }^{284}$ However, adsorbate states are strongly coupled to the metal surface, where electrons are readily available. Adsorbate-induced states have widths on the order of eV, ${ }^{169,277}$ and the corresponding rate of electron jumps is then of the order $10^{15} / \mathrm{s}$; therefore the electrons respond adiabatically to the configurational changes of the reaction intermediates along the reaction pathway. ${ }^{278}$

One motivation for the hypothesis behind rate-limiting electron transfers is the experimental observation of a lower onset potential ( $v s$. RHE) for $\mathrm{C}_{2+}$ products with increased $\mathrm{pH},{ }^{164,286}$ which is consistent with the analysis of the $\mathrm{pH}$ independence of ethylene formation on the SHE scale in the seminal work by Hori. ${ }^{160}$ The idea is that a rate-limiting electron transfer decoupled from proton transfer would show an absolute potential (e.g., vs. SHE) dependence, which is invariant with $\mathrm{pH}$ since the chemical potential of protons is not involved. Absolute potential dependence then translates to more positive onsets on an RHE scale, which accounts for $\mathrm{pH}$. However, dependence on the absolute potential can also arise from either proton-electron transfer from $\mathrm{H}_{2} \mathrm{O}$, which is the proton source in neutral to basic solution, or through field-stabilization of chemical steps with large dipoles. Indeed, hydrogen evolution current densities from metal surfaces have been observed to depend only on SHE potential under a range of $\mathrm{pH}$ from neutral to very alkaline conditions, ${ }^{219}$ which also translates to earlier onsets as $\mathrm{pH}$ is increased.

The effects of $\mathrm{pH}$ on $\mathrm{C}_{2}$ vs. $\mathrm{C}_{1}$ selectivity and activity for $\mathrm{COR}$ and $\mathrm{CO}_{2} \mathrm{R}$ on $\mathrm{Cu}(211)$ was examined in a kinetic model that was based on ab initio activation energies and mean-field adsorbate- 
adsorbate interactions. ${ }^{213}$ In general, as discussed in Subsection 3.3.1, the $\mathrm{C}_{2+}$ activity is enhanced at high $\mathrm{pH}$ and only shows a dependence on the absolute potential, while the $\mathrm{C}_{1}$ activity shows a lesser enhancement with increasing $\mathrm{pH} .{ }^{160,201} \mathrm{H}_{2}$ formation was shown to exhibit a similar SHE dependence to $\mathrm{C}_{2}$ activity. ${ }^{201}$ The kinetic model suggested that the pure SHE dependence of $\mathrm{C}_{2}$ and $\mathrm{H}_{2}$ activities was due to an early rate-determining proton-electron transfer $(* \mathrm{OCCO}$ to $* \mathrm{OCCOH}$, and the Volmer step, respectively) while the mixed RHE/SHE dependence of $\mathrm{C}_{1}$ activity was due to a later proton-electron transfer $\left({ }^{*} \mathrm{CHOH}\right.$ to $\left.{ }^{*} \mathrm{CH}\right)$. The latter is in line with the hypotheses from Hori's original study. ${ }^{160}$ Figure 17 shows the experimental ${ }^{201}$ and theoretical ${ }^{213}$ curves for $\mathrm{CO}$ reduction at $\mathrm{pH} 7$ and $13 .{ }^{213}$ Overall, the major qualitative features between experiment and theory are in agreement. The depletion of $\mathrm{C}_{2}$ products at high overpotentials arises from a decreased $\mathrm{CO}$ coverage upon the onset of $\mathrm{C}_{1}$ production; since $\mathrm{C}_{2}$ production rates are $2^{\text {nd }}$ order in CO coverage, $\mathrm{C}_{2}$ activity is affected significantly more than $\mathrm{C}_{1}$ products by decreases in coverage. This effect is worsened with mass transport limitations. The branching out of the $\mathrm{C}_{1}$ activity at high overpotentials is due to a transition to a regime where the $* \mathrm{CO}$ to ${ }^{*} \mathrm{CHO}$ electrochemical step is rate limiting, in line with the conclusions from Liu et al. ${ }^{169}$
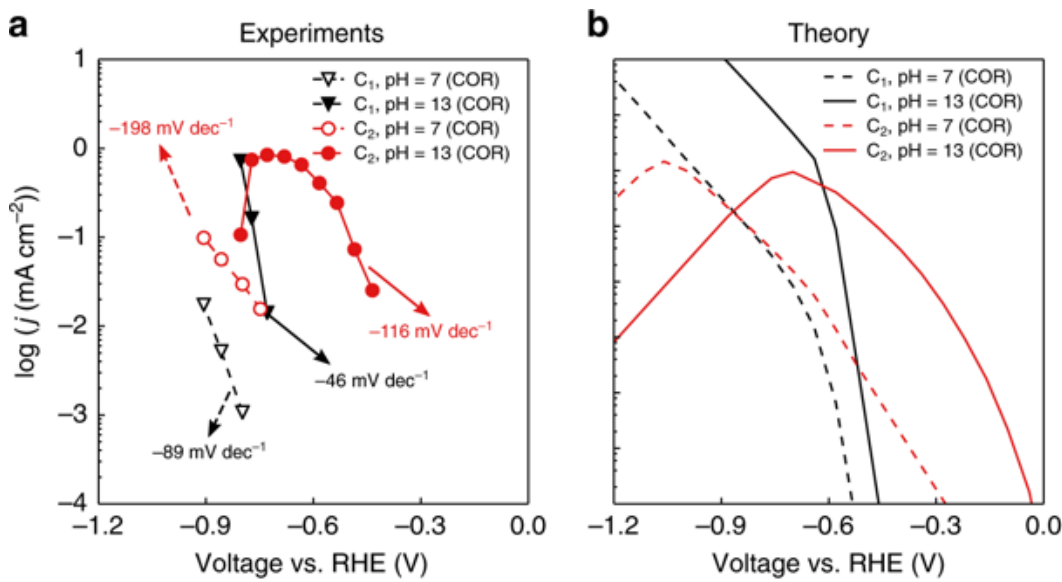

Figure 17: a) Measured COR activities towards $\mathrm{C}_{1}$ and $\mathrm{C}_{2+}$ products on polycrystalline $\mathrm{Cu}$ at $\mathrm{pH} 7$ and 13. Data from Ref. ${ }^{201}$ b) Predicted COR polarization curves from microkinetic model at $\mathrm{pH}=7$ and $\mathrm{pH}=13$. Reprinted from Liu et al. ${ }^{213}$ This work is licensed under a Creative Commons Attribution 4.0 International License (https://creativecommons.org/licenses/by/4.0/).

Xiao et al. also investigated the energetics of reaction pathways on $\mathrm{Cu}(111)$ as a function of $\mathrm{pH}$, using a hybrid of $\mathrm{H}$-shuttling and implicit solvent models. ${ }^{262} \mathrm{~A} \mathrm{pH}$ dependence was added by correcting proton-electron transfer barriers with the shift in the chemical potential of a proton with $\mathrm{pH}$, 
$0.06 \mathrm{eV} / \mathrm{pH}$ unit (some examples given in Table S3 in SI). However, as discussed above, under neutral and alkaline conditions, the source of protons is water, such that the electrochemical barriers would show an absolute (SHE) potential dependence, translating to lower activation energies at higher $\mathrm{pH}$. As a result of the model's $\mathrm{pH}$ correction, under alkaline conditions, all $\mathrm{H}$ transfers are less favored relative to $\mathrm{C}-\mathrm{C}$ coupling. The main conclusion from this analysis was a higher $\mathrm{C}_{2}$ selectivity at high $\mathrm{pH}$, in accordance with experiment, but the assumptions behind the model applied require further examination.

\subsection{Summary}

In recent years, developments in atomistic modeling of the electrochemical interface have made the analysis of $\mathrm{CO}_{2} \mathrm{R}$ pathways possible. Major advances in the field include the establishment of $\mathrm{CO}$ as a crucial reaction intermediate, investigations of cation promotion, the determination of the mechanism towards $\mathrm{CH}_{4}$, where results from different explicit solvent methods appear to converge, and the establishment of design criteria for more active catalysts. On the other hand, there have thus far been little consensus in the theoretical literature regarding $\mathrm{C}_{2}$ reaction pathways and their dependence on $\mathrm{pH}$ and facet termination, due to their inherent complexity, the sensitivity of the intermediates to the solvent and cation environment, and controversy regarding the origin of $\mathrm{pH}$ effects. Experimental evaluation of facet dependence is also challenging due to the possibility of surface reconstruction and defects in single crystal or epitaxial films, as discussed in Section 3.1. We suggest that, while the analysis of onset potentials from reaction energetics can offer significant insight, kinetic modeling is required to elucidate the interplay between coverage, potential dependence of activation energies, adsorbate-adsorbate interactions, and pH effects. Furthermore, we would like to highlight the need for development of multi-scale models ${ }^{287}$ that can help bridge quantum- and molecular-level dynamics with continuum models of reactant and product transport. Additional theoretical hurdles, not limited to investigations of $\mathrm{CO}_{2} \mathrm{R}$, are the reconciliation of differences in conclusions amongst existing theoretical approaches, the evaluation of the simplifying approximations made, and also development of new functionals for improved descriptions of charge at the interface, which would have major implications on the accuracy of the associated energetics. 


\section{Electrochemical $\mathrm{CO}_{2}$ Reduction Pathways on $\mathrm{Cu}$}

Ever since Hori's discovery in 1985 that $\mathrm{CO}_{2}$ is reduced to hydrocarbons on $\mathrm{Cu}$ electrodes with relatively large Faradaic efficiencies, ${ }^{82}$ many researchers have put a lot of effort into elucidating the reaction mechanisms of $\mathrm{CO}_{2} \mathrm{R}$ on $\mathrm{Cu}$. However, after three decades of work, there is still little consensus. Understanding the mechanisms by which products are formed during $\mathrm{CO}_{2} \mathrm{R}$ is essential for the development of effective catalysts that can better meet our needs. Therefore, at this point, we would like to present our current state of understanding of the mechanistic pathways for $\mathrm{CO}_{2} \mathrm{R}$ on $\mathrm{Cu}($ Figure 18), which takes into account a variety of both experimental and theoretical studies described in the previous two chapters.

First we'll provide a few brief notes about the structure and flow of this figure. We have drawn the intermediates in a way that consistently gives each carbon atom a valency of 4 , even though this sometimes differs slightly from the way the intermediate was drawn when proposed. The number on the horizontal axis is the number of carbons in an intermediate or product. The number on the vertical axis is the number of electrons transferred to the intermediate or product, which is four times the number of carbon atoms minus the total oxidation state of the species. The total oxidation state is taken to be the sum of the formal oxidation states of the carbon atoms plus the number of surface-oxygen bonds, representing the fact that bound oxygen has to be reductively cleared from the surface to complete a catalytic cycle. ${ }^{202}$ The formal oxidation of each carbon atom is defined as the sum of the contributions from each of the four bonds: C-H bonds contribute -1 to the carbon atom's oxidation state, C-O bonds contribute $+1, \mathrm{C}-\mathrm{C}$ bonds and $\mathrm{C}$-surface bonds contribute 0 , and an unbound electron pair contributes 1. A dotted bond contributes exactly half as much to the carbon atom's oxidation state as a full bond. Note that these rules are a formalism which actual charge distribution does not rigorously obey. When named, intermediates are generally referred to using the name of their desorbed state. For a bound intermediate to desorb, a pair of bonds to the surface are replaced by an additional bond in the molecule, enabling tautomerization if a C-C double bond is formed adjacent to an $\mathrm{OH}$ group. ${ }^{66}$ If an intermediate with an odd total oxidation state were to desorb from the surface without an electron transfer, it would form a free radical. 
Concerted proton-electron transfer (CPET) steps are represented by solid lines going down one level, while dashed lines involve no proton transfer, dash-dotted lines are coupling reactions involving neither proton nor electron transfer, and dotted lines involve multiple electron transfers. To simplify the figure, we have attempted to group mechanistic proposals that are consistent with each other, also considering which research groups have supported or contributed to each other's proposed mechanisms. The remainder of this chapter summarizes the key reasoning behind the proposed mechanisms based on the experimental and theoretical studies discussed in the previous two chapters. In short: the mechanism in black was proposed by Hori based on his own and other early research in the field. ${ }^{85,160}$ The mechanism in red includes a one-carbon $\left(\mathrm{C}_{1}\right)$ pathway ${ }^{202}$ and initial C-C coupling mechanism ${ }^{234}$ proposed based on DFT calculations by the group of Jens Nørskov, and a $\mathrm{C}_{2}$ pathway based on experimental work from Thomas Jaramillo's group. ${ }^{66}$ The mechanism in blue was proposed by the group of Marc Koper ${ }^{16}$ based on a combination of their experimental observations ${ }^{113}$ and theoretical calculations, ${ }^{284}$ as well as much of the $\mathrm{C}_{1}$ pathway suggested by the group of Michael Janik. ${ }^{264}$ The mechanisms in purple are works by the Goddard group. ${ }^{211,275}$ The orange lines represent a mechanism combining $2 \mathrm{e}^{-}$product pathways from a combined theory-experiment study; ${ }^{288}$ and $>2 \mathrm{e}^{-} \mathrm{C}_{1}$ and $\mathrm{C}_{2}$ pathways determined from microkinetic modeling. ${ }^{213}$ Finally, the green lines depict recent reports on $2 \mathrm{e}^{-}$pathways ${ }^{135}$ and $\mathrm{C}_{2}$ pathways. ${ }^{208}$ Keep in mind that the division of mechanistic proposals in Figure 18 is not absolute or complete. For example, there are many individual theoretical studies described in Chapter 4 that only overlap with certain portions of more extensive mechanistic frameworks shown here. Furthermore, the field is developing quickly, with researchers often adding, removing, and adjusting proposed pathways. Thus, for clarity, the goal of this figure is not meant to be entirely exhaustive in representing all references and the divergences between them, but rather to show several schools of thought that have evolved over time. It is also worth noting that a few other reports have similarly provided mechanistic overviews in recent years. ${ }^{8,276,289,290}$ 


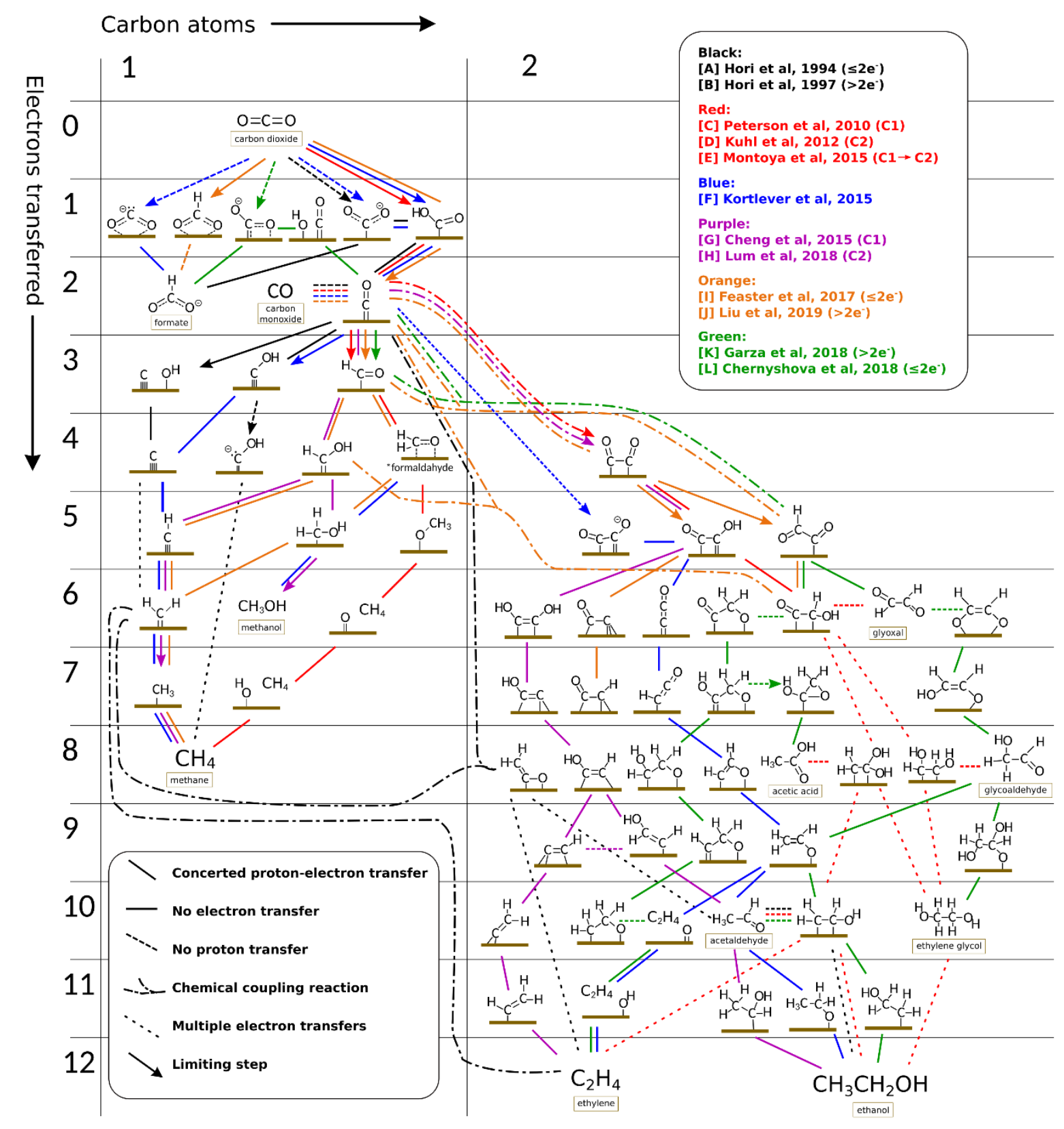

Figure 18: Possible mechanistic pathways of $\mathrm{CO}_{2}$ reduction to $\mathrm{C}_{1}$ and $\mathrm{C}_{2}$ products on polycrystalline copper, grouped into different colored reaction schemes taken from the works in the top-right legend: [A] Hori et al. ${ }^{85}$; [B] Hori et al. ${ }^{160}$; [C] Peterson et al. ${ }^{202}$; [D] Kuhl et al. ${ }^{66}$; [E] Montoya et al. ${ }^{234}$; [F] Kortlever et al. ${ }^{16} ;[\mathrm{G}]$ Cheng et al. ${ }^{278} ;[\mathrm{H}]$ Lum et al. ${ }^{211}$; [I] Feaster et al. ${ }^{288} ;[\mathrm{J}]$ Liu et al. ${ }^{213} ;[\mathrm{K}]$ Garza et al. ${ }^{208}$; [L] Chernyshova et al. ${ }^{135}$. The bottom-left legend states the meaning of the texture of the lines connecting intermediates. Expanded from the mechanistic compilation by S. Scott. ${ }^{291}$

In general, most mechanisms agree that the step in which $\mathrm{CO}_{2}$ is adsorbed is the limiting step in $\mathrm{CO}_{2} \mathrm{R}$ to $2 \mathrm{e}^{-}$products, but there is some uncertainty on whether this first intermediate is arrived at by CPET or a decoupled pathway through a charged intermediate. ${ }^{16}$ Then, whether this intermediate binds through the carbon atom or oxygen atom(s) is thought to be a key distinction between the formate and 
CO pathways. A recent combined experimental and theoretical study found clear volcano trends for the * $\mathrm{COOH}$ binding energy and $* \mathrm{OCHO}$ binding energy for $\mathrm{CO}_{2} \mathrm{R}$ to $\mathrm{CO}$ and $\mathrm{HCOO}^{-}$, respectively, indicating that these are likely the main intermediates for these products. ${ }^{288}$ Alternatively, a recent report combining operando surface-enhanced Raman scattering (SERS), and DFT simulations provide evidence that the first intermediate to both $\mathrm{HCOO}^{-}$and $\mathrm{CO}$ on $\mathrm{Cu}$ electrodes is a carboxylate anion ${ }^{*} \mathrm{CO}_{2} \cdot{ }^{-135}$ However, rather than being bound through the carbon atom or the oxygen atoms, as shown above, the authors propose that carboxylate is coordinated to the surface through one of its C-O bonds, rigorously using isotope exchange and electrochemical Stark effects to verify the assignment of relevant SERS peaks. Then, DFT simulations suggest that this intermediate is converted into $\mathrm{HCOO}^{-}$and $\mathrm{CO}$ via activation of the $\mathrm{Cu}-\mathrm{C}$ and $\mathrm{C}-\mathrm{O}$ bonds and stabilization of the $\mathrm{Cu}-\mathrm{O}$ bond, respectively, with increasing negative potential. ${ }^{135}$ Thus, while different intermediates are proposed, evidently both the $\mathrm{Cu}-\mathrm{C}$ and $\mathrm{Cu}-\mathrm{O}$ bond strengths may still serve as key descriptors governing the selectivity of $\mathrm{Cu}$ to both $\mathrm{CO}$ and $\mathrm{HCOO}^{-}$.

As discussed earlier, it is widely accepted that $\mathrm{CO}$ is the key intermediate to $>2 \mathrm{e}^{-}$products. Beyond $* \mathrm{CO}$, a significant distinguishing feature between the $\mathrm{C}_{1}$ and $\mathrm{C}_{2+}$ pathways is the observed sensitivity to $\mathrm{pH}$. It has been shown that the ethylene formation pathway is $\mathrm{pH}$ independent on the SHE scale, whereas the methane formation pathway is $\mathrm{pH}$ dependent on the SHE scale, ${ }^{160,201}$ which leads to the experimental observation of a lower onset potential ( $v s$. RHE) for $\mathrm{C}_{2+}$ products with increased $\mathrm{pH}$. Accordingly, the limiting step in $\mathrm{C}_{1}$ formation has been thought to be $\mathrm{CO}$ protonation either through a ${ }^{*} \mathrm{COH}$ or $* \mathrm{CHO}$ intermediate, ${ }^{113,160,202,264,275,292}$ while the limiting step in $\mathrm{C}_{2+}$ formation has generally been thought to be CO dimerization that is decoupled from proton transfer. ${ }^{2,113,234,260,261,284}$ Theoretical calculations have differed in their view of $* \mathrm{COH} v s . * \mathrm{CHO}$ depending on the computational techniques and assumptions. A recent microkinetic model has suggested that this $\mathrm{C}_{1}$ rate-limiting step actually changes under different applied potentials, with $* \mathrm{CO} \rightarrow * \mathrm{CHO}$ being limiting at high overpotentials and $* \mathrm{CHO} \rightarrow * \mathrm{CHOH}$ being limiting at low overpotentials. ${ }^{213}$ Furthermore, contrary to the above, this microkinetic model has also suggested that the $\mathrm{C}_{2+}$ pathway could indeed be limited by a CPET step, since the first proton-electron transfer from water to an adsorbate would yield a pure dependence on the 
absolute potential (i.e., it is $\mathrm{pH}$ independent on the SHE scale). ${ }^{213}$ However, it is equally suggested that this $\mathrm{pH}$ independence could also arise from field-stabilization of a limiting chemical reaction with large dipoles (i.e., CO dimerization), which is supported by studies investigating cation effects. This analysis highlights the need for future theoretical studies to consider not only thermodynamics but also reaction kinetics due to the complicated interplay between adsorbate coverages, adsorbate-adsorbate interactions, field effects, etc.

While CO dimerization has been widely suggested, there are many aspects to this step that are still up for debate, such as whether CO coupling is an electrochemical step (involving a rate-limiting electron transfer) $)^{2,113,234,261,284}$ or is purely a chemical step (not involving a rate-limiting electron transfer) $)^{234,261}$ and whether coupling occurs between two adsorbed $\mathrm{CO}$ molecules $\left({ }^{*} \mathrm{CO}+\right.$ $* \mathrm{CO})^{2,113,234,260,261}$ and/or an adsorbed and a free $\mathrm{CO}$ molecule $\left(* \mathrm{CO}+\mathrm{CO}_{(\mathrm{g})}\right) \cdot{ }^{208,284}$ Additionally, some studies also include the possibility of coupling between species such as ${ }^{*} \mathrm{CHO}$ and $* \mathrm{CO}^{261}$ or $\mathrm{CO}_{(\mathrm{g})},{ }^{208,260}$ particularly at higher overpotentials. This partially stems from the notion that there are two possible pathways to ethylene depending on the surface structure and overpotential. A series of studies on $\mathrm{Cu}$ single crystals have noted a low overpotential pathway ( $\mathrm{CO}$ dimerization) preferred on $\mathrm{Cu}(100)$ and a higher overpotential pathway shared with $\mathrm{CH}_{4}$, likely through $* \mathrm{CHO}$, that is preferred on $\mathrm{Cu}(111) .{ }^{164,166,167}$ This is consistent with the higher selectivity for $\mathrm{C}_{2} \mathrm{H}_{4}$ observed on $\mathrm{Cu}(100)$ compared to $\mathrm{Cu}(111)$, as discussed in Subsection 3.1.1. Furthermore, a recent study employing in-situ Fouriertransform infrared spectroscopy (FTIR) observed a hydrogenated CO dimer intermediate at low overpotentials on the $\mathrm{Cu}(100)$ surface but not $\mathrm{Cu}(111)$, providing strong support for this mechanism. ${ }^{141}$ While the authors assigned this species to be ${ }^{*} \mathrm{COCOH}$ based on their DFT calculations, another theoretical study ${ }^{208}$ suggested that, since the vibrational frequencies of adsorbates depend strongly on their adsorption mode, their calculated ${ }^{*} \mathrm{COCHO}$ structure could also have signals in the same range. Thus, discussions continue as to whether the CO dimerization pathway proceeds through a $* \mathrm{COCHO}^{208,234,261}$ or $* \mathrm{COCOH}^{141,260,284}$ intermediate.

Finally, it is also worth mentioning that alternate $\mathrm{C}_{2+}$ mechanisms have been proposed involving dimerization of $\mathrm{CH}_{2}$ groups, ${ }^{104,160,276,293}$ dimerization of $\mathrm{CH}_{3}$ groups, ${ }^{294}$ coupling of two hydrogenated 
CO species, ${ }^{276,281}$ and a Fischer-Tropsch-like $\mathrm{CO}$ insertion mechanism of adsorbed $\mathrm{CH}_{2}$ and $\mathrm{CO} .{ }^{130,160,293}$ While these may be thermodynamically feasible, it is suggested that the surface concentration of these intermediates is likely to be too low for them to be a major source of C-C bonds. ${ }^{208}$ However, this could be highly dependent on the reaction conditions, and thus, these possible mechanisms cannot be conclusively ruled out.

Within the $\mathrm{C}_{1}$ pathway, the selectivity between methane and methanol is of particular interest, as methanol is by far the more valuable product (Figure 2), but has proven elusive. As discussed earlier in Subsection 3.2.2, species related to formaldehyde have been thought to serve as intermediates for methane and methanol formation. One plausible pathway is $* \mathrm{CHO} \rightarrow * \mathrm{CH}_{2} \mathrm{O} \rightarrow * \mathrm{CH}_{3} \mathrm{O} \rightarrow \mathrm{CH}_{3} \mathrm{OH}$ or $\mathrm{CH}_{4}{ }^{202}$ The lack of reduction of methanol to methane ${ }^{113}$ is one indicator that this may not be the most facile route to methane, although the reduction of formaldehyde primarily leads to methanol, ${ }^{108,113,160}$ suggesting that this may be an intermediate on the route to methanol formation. Still, Kuhl et al. observed methane and methanol formation on a number of transition metals and saw that the onset potentials for methane and methanol formation are closely correlated, indicating a mechanism in which these products share a common limiting step. ${ }^{204}$ Cheng et al. (purple $C_{1}$ lines in Figure 18) found that after subsequent protonation of ${ }^{*} \mathrm{CHO},{ }^{*} \mathrm{CHOH}$ is the likely branch point between methanol $\left({ }^{*} \mathrm{CHOH}\right.$ $\left.\rightarrow * \mathrm{CH}_{2} \mathrm{OH} \rightarrow \mathrm{CH}_{3} \mathrm{OH}\right)$ and methane $\left({ }^{*} \mathrm{CHOH} \rightarrow{ }^{*} \mathrm{CH} \rightarrow{ }^{*} \mathrm{CH}_{2} \rightarrow{ }^{*} \mathrm{CH}_{3} \rightarrow \mathrm{CH}_{4}\right)$, with the latter pathway being more favorable. ${ }^{275}$ Thus, $* \mathrm{CO}$ protonation to $* \mathrm{CHO}$ could still apply to both pathways. Interestingly, Kuhl et al. found methanol but no detectable methane on Au (the least oxophilic metal studied) and methane but no detectable methanol on Fe (the most oxophilic metal studied), while the other 5 metals tested $(\mathrm{Ag}, \mathrm{Zn}, \mathrm{Cu}, \mathrm{Ni}, \mathrm{Pt})$ produced both. ${ }^{204}$ Thus, the oxophilicity of the surface may play an important role in determining the selectivity between these two products.

Interestingly, when the selectivity towards methane is increased, a deactivation effect occurs that is not present when ethylene is the major product. ${ }^{110}$ This implies that large amounts of poisoning intermediates form on $\mathrm{Cu}$ in the $\mathrm{C}_{1}$ pathway, and such intermediates may not take part in the $\mathrm{C}_{2+}$ pathway. A likely culprit in this regard is graphitic carbon. Some reports suggest that graphitic carbon can serve as an intermediate in $\mathrm{CO}_{2} \mathrm{R},{ }^{107,264,295}$ and as shown above, a possible pathway to $\mathrm{CH}_{4}$ could go 
through $* \mathrm{COH} \rightarrow{ }^{*} \mathrm{C} \rightarrow$ subsequent hydrogenation to $\mathrm{CH}_{4} \cdot{ }^{264}$ Buildup of graphitic carbon has been commonly suggested as a cause for deactivation though, so this could explain why deactivation is seen on $\mathrm{Cu}$ electrodes that more predominantly make $\mathrm{CH}_{4}$. This could also explain why nanostructured $\mathrm{Cu}$ electrodes, which typically exhibit suppression of the $\mathrm{CH}_{4}$ pathway, have demonstrated increased stability with minimal or no deactivation observed over long term electrolysis. ${ }^{90,108,116,117}$ In addition, the increased surface area of nanostructured electrodes can itself be a cause for their increased stability since the higher ratio of ECSA to electrolyte volume can reduce the impact of poisoning via other mechanisms, such as deposition of trace metal impurities in the electrolyte. ${ }^{88,110,115}$ Furthermore, the surface area of $\mathrm{Cu}$ electrodes also plays a key role in determining selectivity, which will be addressed in the next chapter.

As can be seen in the right half of Figure $\mathbf{1 8}$ above, the proposed mechanisms for $\mathrm{C}_{2}$ products diverge completely after the C-C coupling step, and we refer the reader to the original studies for the detailed reasoning behind each proposed mechanism. ${ }^{16,66,160,208,211,213,234}$ In short, as discussed in Subsection 3.2.2, the reduction of oxygenated species such as glyoxal, glycoaldehyde, acetaldehyde, propionaldehyde, etc. have led to identification of possible intermediates and pathways to alcohols. In addition, water has recently been shown to play an important role in the formation of certain oxygenated products. ${ }^{211}$ Under strongly alkaline conditions, Cannizzaro-type disproportionation reactions in the electrolyte may also lead to the formation of alcohols and carboxylic acids from the corresponding aldehyde. ${ }^{212}$ In terms of potential-dependent trends, some studies have demonstrated that oxygenates are preferred over hydrocarbons at lower overpotentials. ${ }^{115,201}$ Overall, there is still much being learned about what dictates selectivity between the various $\mathrm{C}_{2+}$ oxygenates and hydrocarbons. Through future work developing in-situ/operando spectroscopies, isotope labeling experiments, and computational methods involving kinetic modeling, we may be able to ascertain the distinguishing features of the dominant pathway(s) in order to tailor $\mathrm{CO}_{2} \mathrm{R}$ activity and selectivity towards the desired product(s).

This concludes our brief overview of reaction networks for $\mathrm{CO}_{2} \mathrm{R}$ on $\mathrm{Cu}$. In the chapters that follow, we will expand on this framework and discuss deeper insights on activity \& selectivity trends that have been gained from studying more advanced (nanostructured and bimetallic) $\mathrm{Cu}$ catalysts. 


\section{Nanostructured Copper}

Nanostructuring of $\mathrm{Cu}$ electrodes has been widely applied in attempts to improve selectivity and energy efficiency for $\mathrm{CO}_{2} \mathrm{R}$. Nanostructuring of electrocatalyst materials leads to improved geometric activity (current density normalized to the geometric surface area of the electrode) due to an increase in the electrochemically active surface area (ECSA) compared to planar electrodes. In addition, it can oftentimes lead to altered electrocatalytic properties compared to those of planar electrodes. These differences can be caused by a number of effects, including, but not limited to, a higher occurrence of undercoordinated sites, preferential faceting, changes in the local $\mathrm{pH}$, and re-adsorption of products. ${ }^{296,297}$ In this work, we classify the $\mathrm{Cu}$ nanomaterials into two different types of systems: (supported) nanoparticles (Section 6.1) and (unsupported) three-dimensionally interconnected electrodes (Section 6.2). It is important to note that these two sections both address aqueous phase $\mathrm{CO}_{2} \mathrm{R}$, meaning that $\mathrm{CO}_{2}$ is delivered to the electrode via diffusion through the aqueous electrolyte. Vapor-fed $\mathrm{CO}_{2} \mathrm{R}$ systems, where the $\mathrm{CO}_{2}$ is delivered directly to the electrode in the gas phase, will briefly be addressed in Section 6.3. In this chapter we will only discuss pure $\mathrm{Cu}$ systems, in some cases on a support material; nanostructured bimetallic systems with $\mathrm{Cu}$ are covered in Chapter 7.

\subsection{Nanoparticles}

Nanoparticles are individual particles with a diameter of $100 \mathrm{~nm}$ or less that are typically supported on a suitable material. Since all heterogeneous catalytic processes take place at the surface, the high surface area to volume ratio of nanoparticulate catalysts allows for greater utilization of a given material on a mass basis. This is an important concern for precious metal catalysts, but is less of an issue for $\mathrm{Cu}$ because of its natural abundancy. ${ }^{298}$ An important motivating factor for studying nanoparticle catalysts is their high degree of compatibility to be incorporated into gas diffusion electrodes (GDEs) and membrane electrode assemblies (MEAs); these vapor-fed systems will be discussed in Section 6.3. Here we will detail work that has been done to study the fundamental effects of nanoparticle size, shape, and loading on $\mathrm{CO}_{2} \mathrm{R}$ performance in aqueous-phase reactors. ${ }^{299-307}$ 


\subsubsection{Nanoparticle Size Effects}

Particle size can have a direct influence on the reactivity of a catalyst material. This comes down to a number of possible effects, mainly related to a significant increase in the surface to bulk atom ratio as the size is decreased. Firstly, as the size of a particle decreases the curvature of its surface becomes larger and the average coordination of the surface atoms is lowered, due to a higher occurrence of undercoordinated sites. ${ }^{308}$ Decreasing the coordination number of catalytically active atoms leads to perturbed electronic structure and oftentimes increased reactivity. Furthermore, for very small particles, a significant amount of strain is induced on the surface atoms. This shifts the d-band and, again, influences reactivity. ${ }^{309}$ Depending on the catalytic process and material, increased reactivity may improve or deteriorate activity. For simple $2 \mathrm{e}^{-}$processes, the effect of changes in reactivity can be predicted by determining the optimum binding energy of the key intermediate. ${ }^{205}$ However, $\mathrm{CO}_{2} \mathrm{R}$ pathways to $>2 \mathrm{e}^{-}$products are inherently much more complex (as was shown in Figure 18) and involve many more proton-electron transfers and intermediate species. Therefore, it is hard to predict the effect of changes in the electronic structure on the catalyst performance, and both theory and experimental work are needed.

Based on the concepts outlined above, a few different groups have investigated size effects of $\mathrm{Cu}$ nanoparticles for $\mathrm{CO}_{2} \mathrm{R}^{299-301}$ The results from various individual studies generally show different trends. Importantly, they all used different catalyst synthesis methods, which can lead to different nanoparticle size, morphology and stability. If ligands are used during synthesis, they can bind to active sites and alter the activity if not completely removed before electrochemical testing. Furthermore, $\mathrm{Cu}$ is inherently mobile, in particular in the presence of CO. ${ }^{175}$ Thus, depending on the initial geometry, significant restructuring can take place under reaction conditions, which can cause changes in nanoparticle size, shape, and distribution. 

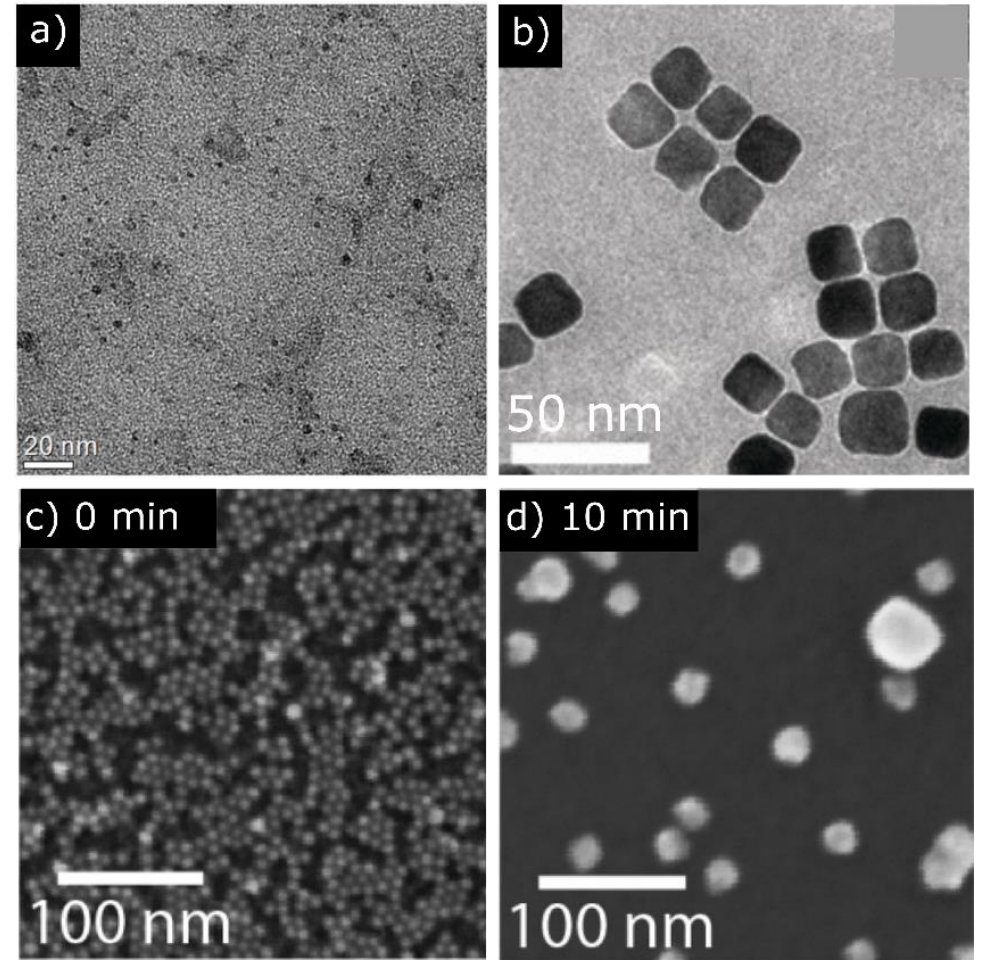

Figure 19: TEM images of $\mathrm{Cu}$ nanoparticles from various studies. a) Spherical particles, $2.4 \mathrm{~nm}$ average diameter. Reprinted from Reske et al. ${ }^{299}$ Copyright (C 2014, American Chemical Society. b) Cubic particles, $24 \mathrm{~nm}$ average edge length, Reprinted with permission from Loiudice et al. ${ }^{301}$ Copyright (C 2016, John Wiley and Sons. c) and d) Spherical particles as-prepared and after $10 \mathrm{~min}$ $\mathrm{CO}_{2} \mathrm{R}$ at $-1.25 \mathrm{~V}$ vs. RHE, respectively. Reprinted from Manthiram et al. ${ }^{300}$ Copyright (C) 2014, American Chemical Society.

Reske et al. synthesized various $\mathrm{Cu}$ nanoparticle electrodes of diameter $15 \mathrm{~nm}$ and smaller. A TEM image of a representative sample is displayed in Figure 19a. When measuring $\mathrm{CO}_{2} \mathrm{R}$, the authors observed that the samples exhibited lower $\mathrm{CO}_{2} \mathrm{R}$ Faradaic efficiency towards hydrocarbons and increased selectivity to the formation of $\mathrm{CO}$ and $\mathrm{H}_{2}$ as compared to polycrystalline foils, as displayed in Figure 20b. ${ }^{299}$ Furthermore, they showed that particles smaller than $5 \mathrm{~nm}$ gave rise to significantly increased current density at a fixed potential, which led to increased production rates for the various products as displayed in Figure 20a. Surprisingly, no clear trend in product distribution could be observed with changing particle size in this study. Manthiram et al., on the other hand, reported enhanced methane production between -0.95 and $-1.45 \mathrm{~V}$ vs. RHE on $\mathrm{Cu}$ nanoparticles with an initial size of $7 \mathrm{~nm}$, as compared to polycrystalline $\mathrm{Cu}^{300}$ These nanoparticles quickly sintered, as illustrated by the transformation over 10 minutes of $\mathrm{CO}_{2} \mathrm{R}$ shown in Figures 19c and 19d. Loiudice et al. 
synthesized larger, cube shaped nanocrystals of $24-63 \mathrm{~nm}$, with a TEM image of a $24 \mathrm{~nm}$ particle sample displayed in Figure 19b. ${ }^{301}$ They showed an optimal $\mathrm{CO}_{2} \mathrm{R}$ Faradaic efficiency of around $80 \%$, with high selectivity toward ethylene, for $44 \mathrm{~nm}$ particles at $-1.1 \mathrm{~V} v$ s. RHE. As mentioned above, the divergence between these three studies can have different reasons. Manthiram et al. showed that their $7 \mathrm{~nm}$ particles aggregated to form larger clusters with an average size of $23 \mathrm{~nm}$ shortly after introduction to the catalytic environment. ${ }^{300}$ Furthermore, two of the studies used spherical particles, ${ }^{299,300}$ while the third mainly studied cube-shaped particles. ${ }^{301}$ As discussed in Section 3.1, differences in surface structure can influence product distribution, and to some extent activity. Finally, different loadings were used, both between the different studies and within a single study. The effect of this will be discussed in the following subsection. Unfortunately, the diverging results make it difficult to draw clear conclusions about trends for different sizes of $\mathrm{Cu}$ nanoparticles.
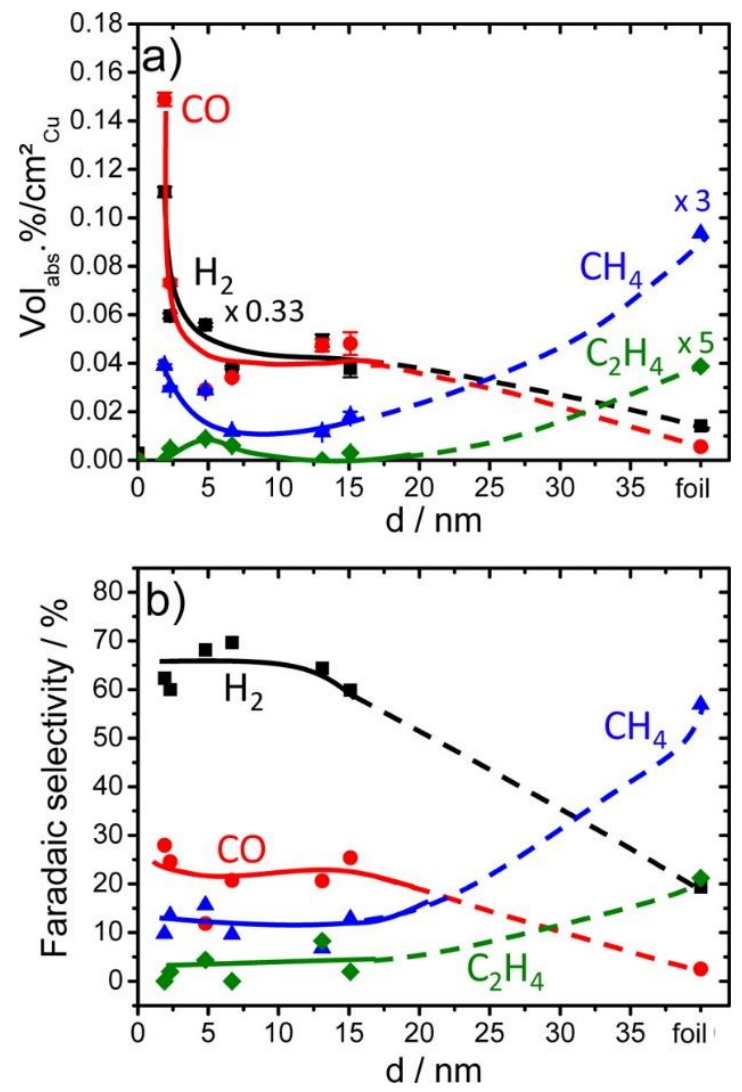

Figure 20: $\mathrm{CO}_{2}$ reduction on $\mathrm{Cu}$ nanoparticles of different size at $-1.1 \mathrm{~V} v s$. RHE. (a) Production rate of individual products. (b) Faradaic efficiency to individual products. Reprinted from Reske et al. ${ }^{299}$ Copyright (C) 2014, American Chemical Society. 


\subsubsection{Loading/Interparticle Distance}

Another parameter that can influence the $\mathrm{CO}_{2} \mathrm{R}$ activity and selectivity of nanoparticles is the amount that is loaded onto the support. ${ }^{302,303}$ Mistry et al. used inverse micelle encapsulation to synthesize nanoparticles with well-defined size and interparticle distance. ${ }^{302}$ They found some variation in the product distribution with loading, depending on particle size, but no clear trend, similarly to what was the case for the study of pure size effects, as discussed above. They attributed the effects to readsorption of products/intermediates and local $\mathrm{pH}$ effects. Kim et al. investigated three widely different loadings of $6.7 \mathrm{~nm} \mathrm{Cu}$ particles on carbon paper. ${ }^{303}$ The highest loading was studied further, leading to observations of a structural transition of nanoparticle ensembles into cube-shaped structures. This coincided with a shift in product distribution towards more $\mathrm{C}_{2+}$ compounds and lower $\mathrm{H}_{2}$ evolution, possibly caused by the exposure of different surface sites and/or local $\mathrm{pH}$ changes in the rough structure. The studies mentioned above should be followed up with further work regarding mass transport effects on $\mathrm{CO}_{2} \mathrm{R}$ activity and selectivity for $\mathrm{Cu}$ nanoparticle systems. Additional well-controlled studies using alternative synthesis techniques and three-dimensional support materials will help to elucidate some of these phenomena.

\subsubsection{Shape Effects}

As discussed in Subsection 3.1.1, certain $\mathrm{Cu}$ single crystal facets exhibit improved selectivity to specific products. $\mathrm{Cu}(100)$ electrodes, and especially "miscut" $\mathrm{Cu}(100)$ electrodes with an optimal density of steps, have been shown to exhibit higher selectivity for C-C coupling than other planar surfaces..$^{154,158,164,167}$ Reports also suggest that the production of oxygenates is enhanced on certain crystals, including single crystals with (100) and (110) terraces and varying step density, as well as epitaxially grown $\mathrm{Cu}$ films with (751) orientation. ${ }^{153,154,158}$ Motivated by the work on single crystals, some effort has gone into increasing the occurrence of certain facets by controlling nanoparticle shape. For instance, the surface of cubic structures should be dominated by (100) facets since $\mathrm{Cu}$ is a facecentered cubic (FCC) metal. In addition, unlike perfect single crystals, faceted nanoparticles also contain edge and corner sites, which could affect the resulting activity strongly. For instance, recent 
DFT results suggest that $\mathrm{Cu}(211)$, which exhibits a high density of surface step sites, yields several orders of magnitude higher activity than (100) and (111) surfaces for $\mathrm{CO}$ reduction to $\mathrm{CH}_{4} \cdot{ }^{169}$

Cubic $\mathrm{Cu}$ nanoparticles have been synthesized by different groups, as discussed in the previous two subsections. ${ }^{301,303,310}$ A representative TEM image is shown in Figure 19b. These particles exhibit enhanced $\mathrm{C}_{2+}$ product selectivity compared to polycrystalline $\mathrm{Cu}$, possibly caused by increased occurrence of (100) facets. However, Grosse et al. reported drastic morphology changes undergoing during $\mathrm{CO}_{2} \mathrm{R}$, including loss of the cubic shape. ${ }^{310} \mathrm{Li}$ et al. produced thin nanowires with well-defined five-fold twinned edges, exhibiting high selectivity towards $\mathrm{CO}_{2} \mathrm{R}$, with $\mathrm{CH}_{4}$ produced almost exclusively around $-1.25 \mathrm{~V} v s$. RHE. ${ }^{304}$ It is unclear, however, which facets are exposed on the terraces of the nanowires. It is also worth mentioning that $-1.25 \mathrm{~V}$ is a large overpotential, where polycrystalline $\mathrm{Cu}$ also mainly produces methane from $\mathrm{CO}_{2} \mathrm{R} \cdot{ }^{66}$ Furthermore, at potentials more negative than $-1.0 \mathrm{~V}$ $v s$. RHE, polarization effects begin to impact $\mathrm{CO}_{2} \mathrm{R}$ catalysis on planar polycrystalline $\mathrm{Cu},{ }^{97}$ meaning that these high surface area nanowire electrodes will likely experience mass transport limitations at even lower applied potentials. We would like to underline the importance of measuring novel catalyst materials at potentials where mass transport limitations are not prevalent in order to more directly investigate their intrinsic behavior; this issue was discussed in more detail in Section 2.2.

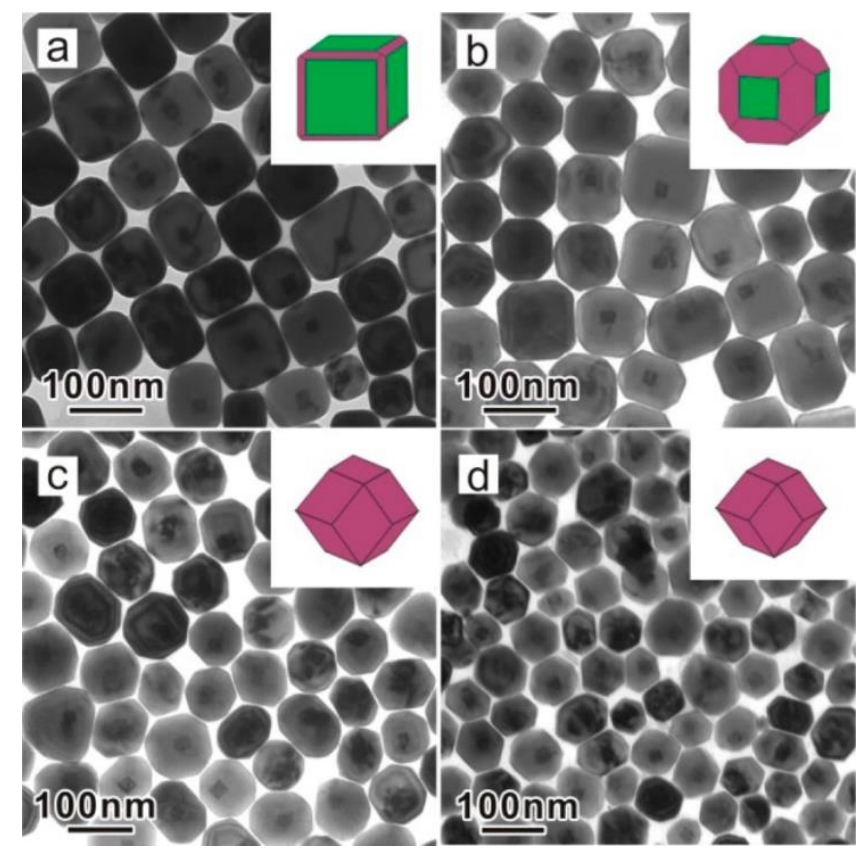

Figure 21: $\mathrm{Cu}$ nanoparticles prepared by etching cubic particles for different durations: a) $4 \mathrm{~h}, \mathbf{b}) 8 \mathrm{~h}$, c) $12 \mathrm{~h}$, and d) $24 \mathrm{~h}$. Reprinted from Wang et al. ${ }^{305}$ Copyright $\odot$ 2016, American Chemical Society. 
The studies mentioned in the previous paragraph utilized bottom-up methods to obtain faceting of their particles. With a different, top-down approach, Wang et al. used chemical etching of cubic $\mathrm{Cu}$ nanoparticles with varying duration to obtain different shapes, as shown in Figure 21. ${ }^{305}$ The nanocrystals that were etched for 12 hours or more (Figures 21c and 21d) exhibited a rhombic dodecahedral shape with preferential exposure of (110) facets. These particles exhibited enhanced selectivity to hydrocarbons and reduced $\mathrm{CO}$ production at around $-0.8 \mathrm{~V} v s$. RHE. The authors did not, however, report any liquid products. As mentioned above, Hori et al. reported that single crystals with (110) orientation exhibit significantly enhanced production of oxygenates. ${ }^{153}$ Therefore, it is likely that oxygenates were produced from these measurements as well. This emphasizes the importance of carrying out thorough product analysis, since this information can give significant fundamental insight. An important point to consider when investigating shape effects is stability, both in terms of activity and retaining the synthesized shape. This is particularly important for $\mathrm{Cu}$, where structural transformations are likely due to its high mobility. In an attempt to address this issue, Huang et al. studied the development of shape and $\mathrm{CO}_{2} \mathrm{R}$ activity of the cubic $\mathrm{Cu}$ nanoparticles shown in Figure 19b with electrolysis time. Using TEM, they performed ex-situ monitoring of changes to the morphology of the nanoparticles after various durations of electrolysis up to $12 \mathrm{~h}$, while also tracking activity and product distribution. They saw some changes with time; $\mathrm{H}_{2}$ evolution activity increased and $\mathrm{CO}_{2} \mathrm{R}$ activity decreased with longer duration for most samples, and the nanoparticle shape became increasingly ill-defined. They attributed the changes to adsorbate-induced restructuring of the $\mathrm{Cu}$ catalyst, yielding useful information for further studies. However, they did not provide conclusive evidence that this is the only or main cause of the deactivation. Importantly, they did not provide evidence that their electrodes were not contaminated, which can be challenging to avoid for such longterm measurements due to the highly negative polarization of the electrode. ${ }^{12,114,115}$ Furthermore, they only reported $\mathrm{CO}_{2} \mathrm{R}$ at $-1.1 \mathrm{~V} v s$. RHE, which is in a potential region where non-planar electrodes are likely to start reaching mass transport limited activity. Thus, any trends in activity that are observed here might not be valid at lower overpotentials. 
In general, more work on nanoparticles with preferential faceting would be welcome, in particular to elucidate the effect of particle shape in more detail, and to be able to decouple it from other parameters such as size, loading, support effects, etc. Further stability studies addressing the points raised in the previous paragraph would also be of value to the community.

\subsubsection{Effect of Support and Catalyst-Ionomer Interaction}

For any nanoparticle-based catalyst, a suitable support material is vital to ensure good dispersion and mechanical stability of the catalyst material. In addition, the interaction between support and nanoparticles can induce changes in the electronic structure of the catalyst and/or add interfacial sites, altering the performance of the catalyst. The support effect is utilized heavily in several industrial catalytic reactions, such as methanol synthesis on $\mathrm{Cu} / \mathrm{ZnO} / \mathrm{Al}_{2} \mathrm{O}_{3}{ }^{311}$ However, only a limited number of studies investigating support effects in $\mathrm{CO}_{2} \mathrm{R}$ have been reported to date. Song et al. showed that $\mathrm{Cu}$ nanoparticles supported on needle-shaped nitrogen doped carbon exhibit high selectivity towards ethanol production at high overpotentials, with a maximum Faradaic efficiency of $63 \%$ at $-1.2 \mathrm{~V} v \mathrm{~s}$. RHE. ${ }^{312}$ They attributed the high selectivity to the interaction between the nanoparticles and the highly textured support material. Subsequently, a number of further studies on $\mathrm{Cu}$ nanoparticles on various carbon supports reported significant selectivity towards ethanol ${ }^{313}$ or ethylene. ${ }^{314,315}$

Feng et al. synthesized $\mathrm{Cu}$ nanoparticles on graphene by physical vapor deposition, and observed a large number of grain boundaries. ${ }^{316}$ From these non-equilibrium structures, they observe high selectivity towards ethanol and acetate from COR. Some works also report catalytic enhancement from metal-support interactions for $\mathrm{CO}_{2} \mathrm{R}$ on $\mathrm{Au}$ and $\mathrm{Ag} .{ }^{317,318}$ TEM images illustrating the geometry of these two studies are shown in Figures 22a and 22b, respectively. 

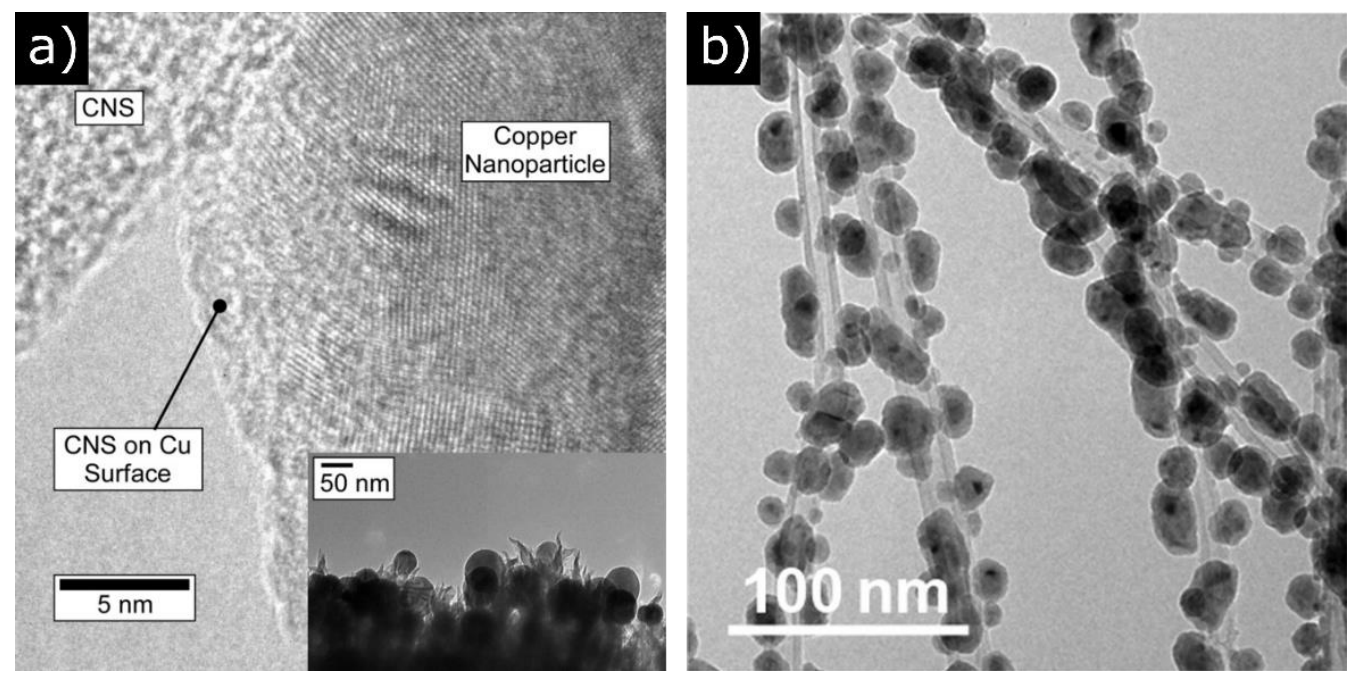

Figure 22: Transmission electron microscopy images of $\mathrm{Cu}$ nanoparticles on two different supports. a) Carbon nanospikes. Reprinted with permission from Song et al. ${ }^{312}$ Copyright $(9) 2016$, John Wiley and Sons. b) Carbon nanotubes. Reprinted with permission from Feng et al. ${ }^{316}$ Further permissions related to this material should be directed to the American Chemical Society.

In moving towards more practical device designs, such as vapor-fed systems (Section 6.3), understanding how the other components including the support and ionomer materials impact $\mathrm{CO}_{2} \mathrm{R}$ on $\mathrm{Cu}$ will become increasingly important. For example, it has previously been shown that an optimal ionomer loading exists for hydrogen oxidation electrocatalysts. ${ }^{319}$ Gupta et al. tried to determine a similar ideal ionomer content for $\mathrm{Cu}$ nanoparticles, and found that optimal formate production occurs using $25 \%$ Nafion content in the catalyst ink. ${ }^{307}$ We would like to highlight the need for more fundamental studies in these areas in order to help translate aqueous-phase $\mathrm{CO}_{2} \mathrm{R}$ catalysis to highly complex vapor-fed designs.

\subsection{Three-Dimensionally Interconnected Catalyst Materials}

Another approach to nanostructuring is the preparation of three-dimensionally interconnected (3-D) electrodes. In the following, we separate the discussion between oxide-derived and non-oxidederived electrodes and consider how the different pretreatments influence $\mathrm{CO}_{2} \mathrm{R}$ activity. 


\subsubsection{Oxide-Derived Copper}

Rapid removal of oxygen during reduction of oxidized $\mathrm{Cu}$ is a straightforward means to achieve porous, inhomogeneous surfaces that exhibit altered $\mathrm{CO}_{2} \mathrm{R}$ performance. Hori et al. were the first, to the best of our knowledge, to report $\mathrm{CO}_{2} \mathrm{R}$ on this type of $\mathrm{Cu}$ electrode, which had significantly higher ECSA, as measured by the double-layer charging current. ${ }^{160}$ Other early work by Karl W. Frese Jr. reported significant methanol production from $\mathrm{CO}_{2} \mathrm{R}$ on intentionally oxidized copper electrodes. ${ }^{320} \mathrm{~A}$ large number of later works on similar catalysts have not reported methanol production; thus, it is still not clear how, if at all, methanol can be produced with significant selectivity from $\mathrm{Cu}$ electrodes. However, the preparation of nanostructured surfaces from oxidized precursors has still led to a number of interesting findings, which will be discussed subsequently. For ease of navigating the wide variety of materials that have been studied, we organize this subsection by the choice of synthesis method: thermal oxidation, anodic treatment, and other $\mathrm{Cu}_{2} \mathrm{O}$ film-derived electrodes.

\section{Thermal oxidation}

Kanan and coworkers showed that calcination of planar $\mathrm{Cu}$ foils in air and subsequent electrochemical reduction resulted in high surface area 3-D electrodes.$^{90}$ An SEM image that illustrates the resulting surface structure can be seen in Figure 23a. When carrying out $\mathrm{CO}_{2} \mathrm{R}$ on these electrodes, the selectivity towards $\mathrm{CO}$ and formate was improved between -0.2 and $-0.7 \mathrm{~V} v s$. RHE, compared to polycrystalline $\mathrm{Cu}$ foil. Similar studies have since been carried out using this thermal oxidation and electrochemical reduction method to alter the $\mathrm{CO}_{2} \mathrm{R}$ activity of $\mathrm{Cu}$ electrodes, but a common approach has been to use a copper precursor material with a higher surface area than planar foils, such as $\mathrm{Cu}$ mesh, foam, and nanowire structures..$^{92-94,321,322}$ Some of these studies report enhanced production of $\mathrm{CO}$ and formate, and little to no formation of $>2 \mathrm{e}^{-}$products. ${ }^{92-94}$ This corresponds well to the results by Kanan and coworkers. ${ }^{90}$ On the other hand, two studies also showed enhanced selectivity for $>2 \mathrm{e}^{-}$ products, such as hydrocarbons and alcohols, compared to polycrystalline $\mathrm{Cu}$, which was not reported previously. ${ }^{321,322}$ Notably, $\mathrm{CH}_{4}$ production was suppressed in favor of $\mathrm{C}-\mathrm{C}$ coupled products. Possible causes for the discrepancy between these studies on calcined oxide-derived $\mathrm{Cu}$ electrodes could be 
inadequate product analysis in some of the studies and varying mass transport conditions within the different catalyst morphologies and electrochemical cells used by the different research groups.

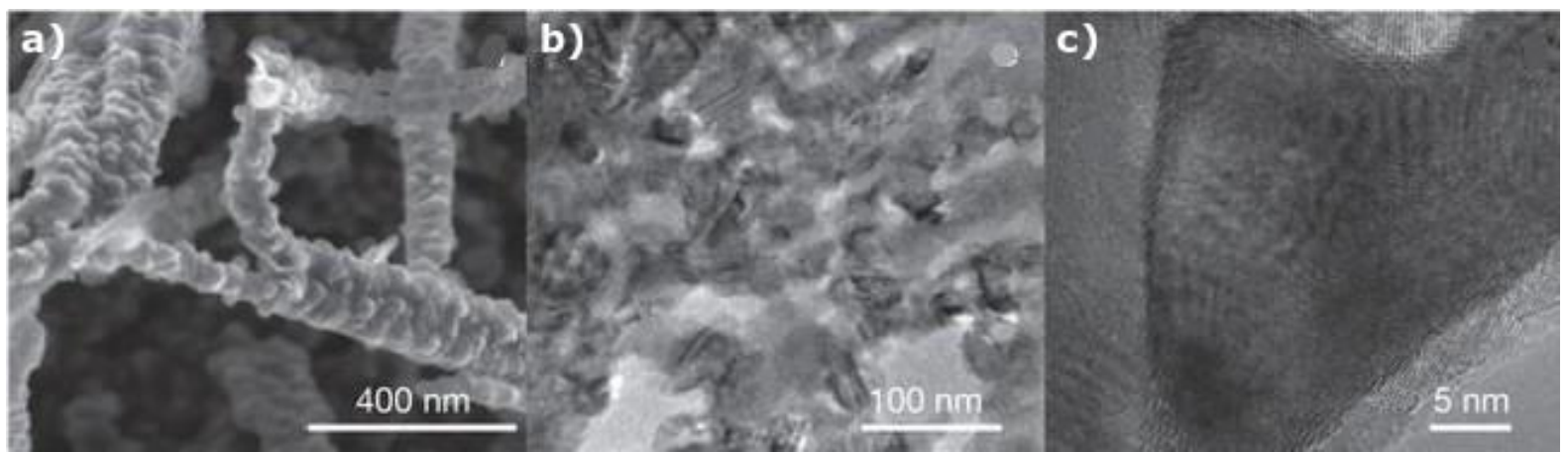

Figure 23: Electron microscopy images of calcined oxide-derived Cu electrodes. a) and b) SEM images. c) TEM image. Reprinted with permission from Li et al. ${ }^{71}$ Copyright (C) 2014, Springer Nature.

Kanan and coworkers also conducted $\mathrm{CO}$ reduction $(\mathrm{COR})$ studies on their oxide-derived $\mathrm{Cu}$ foils. ${ }^{71}$ High COR selectivity was observed between -0.25 and $-0.40 \mathrm{~V} v s$. RHE, mostly towards the $\mathrm{C}_{2}$ compounds ethanol and acetate. At $-0.3 \mathrm{~V}$ vs. RHE, CO was reduced at a Faradaic efficiency of 57\%, with an impressive $43 \%$ to ethanol production. Similarly high activity and selectivity towards COR was reported by Raciti et al. on Cu nanowires. ${ }^{323}$ However, due to the poor solubility of carbon monoxide in aqueous solutions, ${ }^{324}$ low maximum COR current densities were obtained in both of these studies. Nevertheless, such a high selectivity towards liquid products spurred much interest in this class of materials..$^{91,316,325,326}$

In the aforementioned COR study by Kanan and coworkers, a large number of grain boundaries in the calcined oxide-derived $\mathrm{Cu}$ electrodes were found using transmission electron microscopy (TEM), as illustrated in Figures $\mathbf{2 3 b}$ and $\mathbf{2 3 c}$. $^{71}$ They hypothesized that the high selectivity could be caused by undercoordinated sites with special geometries that were stabilized by grain boundary surface terminations. By later studying nanoparticles with varying grain boundary density, they were able to correlate the COR performance to a high occurrence of grain boundaries. ${ }^{316}$ Verdaguer-Casadevall et al. used CO temperature programmed desorption (TPD) to probe the binding strength of CO on oxidederived $\mathrm{Cu}$ foils. ${ }^{325} \mathrm{~A}$ high temperature feature that was not present for planar polycrystalline $\mathrm{Cu}$ could be identified, suggesting the presence of strong-binding sites on the oxide-derived surface. The 
occurrence of these sites could be correlated with COR activity, supporting the notion that certain sites present on oxide-derived $\mathrm{Cu}$ are responsible for its excellent performance. However, the TPD in this study was complicated by gas-phase mass transport and $\mathrm{CO}$ re-adsorption in the pores of the oxidederived copper, and modeling was required to decouple this effect from the binding strength of particular sites. The modeling suggested that the high temperature feature was entirely due to strong binding of $\mathrm{CO}$, rather than mass transport, but there is still some uncertainty to this result due to the inhomogeneity of the material.

Rather than specific morphological features being responsible for the high COR activity and selectivity to oxygenated products, other studies have suggested that this has more to do with the increased surface area of these materials. ${ }^{15,201}$ As shown in Figure 24, the production of both oxygenated and $\mathrm{C}-\mathrm{C}$ coupled products are favored at lower overpotentials. ${ }^{201}$ Thus, the performance of these very rough morphologies can be explained by their greater number of surface sites, which allow these electrodes to reach high geometric COR rates at more positive potentials where the production of $\mathrm{C}_{2+}$ oxygenates is favored. Importantly, when normalized by the ECSA, planar $\mathrm{Cu}^{201}$ and these state of the art $\mathrm{Cu}$ materials ${ }^{71,323}$ exhibit very similar $\mathrm{COR}$ rates when compared at potentials that are free of diffusion limitations, suggesting that these potential-dependent selectivity trends are the key reason for the differing performance of these materials.
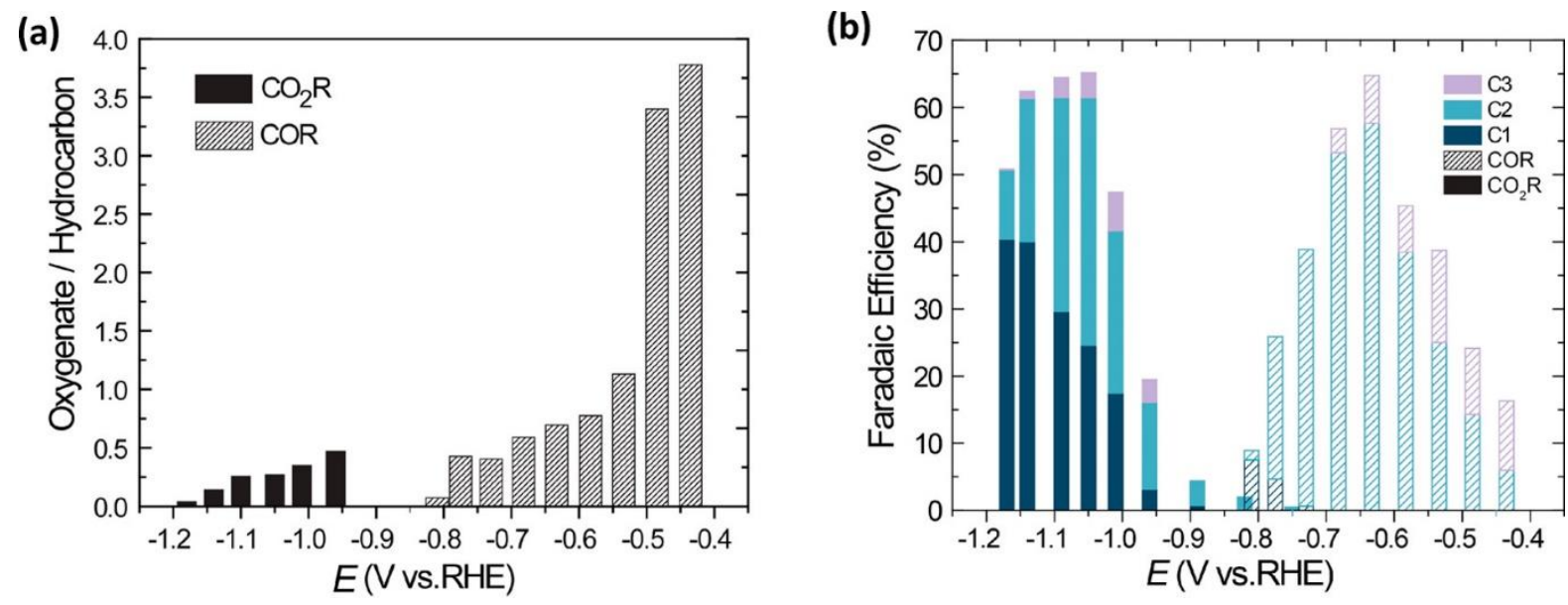

Figure 24: Potential-dependent selectivity of $\mathrm{Cu}$ toward $\mathrm{CO}_{2} \mathrm{R}$ and $\mathrm{COR}$. (a) oxygenate/hydrocarbon ratios of the reduction products as a function of potential, and (b) Faradaic efficiencies for $\mathrm{C}_{1}, \mathrm{C}_{2}$, and $\mathrm{C}_{3}$ products, each as a function of potential. These potential-dependent selectivity trends reveal that the production of $\mathrm{C}_{2+}$ oxygenates is increasingly favored over hydrocarbons at Reprinted from Wang et al. ${ }^{201}$ Copyright $\odot$ 2018, American Chemical Society. 


\section{Anodic treatment}

Another approach to prepare oxide-derived nanostructured electrocatalysts is in-situ anodic treatments. Oxidation-reduction cycles result in the formation of nanostructures on the surface of polycrystalline $\mathrm{Cu} .^{95,96,117,327}$ Similar to some studies on calcined oxide-derived $\mathrm{Cu}$, the in-situ electrochemical oxidation-reduction treatment resulted in suppressed $\mathrm{CH}_{4}$ evolution and enhanced production of $\mathrm{C}_{2} \mathrm{H}_{4}$ compared to planar $\mathrm{Cu}$. $\mathrm{C}_{2}$ and $\mathrm{C}_{3}$ oxygenates were later identified as important liquid products from anodically treated $\mathrm{Cu}$ foils by performing rigorous product analysis. ${ }^{96,328}$ Roberts et al. followed up their $\mathrm{CO}_{2} \mathrm{R}$ work on copper nanocubes synthesized by potential cycling in the presence of $\mathrm{Cl}^{-95}$ by also testing these anodically nanostructured catalyst for COR. ${ }^{286}$ Ethylene formation was favored over methane on copper nanocubes compared to polycrystalline foils, confirming the trends they observed previously for $\mathrm{CO}_{2} \mathrm{R}$. In general, it seems that anodic nanostructuring of $\mathrm{Cu}$ surfaces favors production of $\mathrm{C}_{2+}$ over $\mathrm{C}_{1}$ compounds. Using a different approach, Roldan Cuenya and coworkers created a thick, amorphous oxide film on the surface of $\mathrm{Cu}$ foils by oxygen plasma treatment. ${ }^{126,249,329}$ Depending on the exact pre-treatment conditions, this material exhibited high selectivity towards ethylene formation. A maximum Faradaic efficiency of $62 \%$ was observed at around $-0.9 \mathrm{~V} v$ s. RHE. ${ }^{126}$

Different reasons for the high ethylene selectivity of these materials have been suggested. Kwon et al. ${ }^{96}$ reiterated the explanation given by Kanan and coworkers for calcined oxide-derived copper, attributing it to the high occurrence of grain boundaries and/or other defects on the surface. ${ }^{316}$ In the case of the copper nanocubes, it has been speculated that the high degree of (100) surface orientation of the electrodes could play a role, due to the cubic geometry of the surface crystallites and the large number of undercoordinated sites present. ${ }^{95}$ Eilert et al. used X-ray absorption spectroscopy to study the properties of the $\mathrm{Cu}$ electrodes as they were undergoing redox cycling to produce the cube-shaped surfaces, showing that these nanostructures are derived from $\mathrm{Cu}_{2} \mathrm{O} .{ }^{125}$ They also prepared a catalyst derived from a $\mathrm{CuO}$ precursor, exhibiting very similar $\mathrm{CO}_{2} \mathrm{R}$ performance. Interestingly, these electrodes showed a disordered surface morphology without cubic geometries. This points towards other causes for the high $\mathrm{C}_{2} \mathrm{H}_{4} / \mathrm{CH}_{4}$ ratio than the occurrence of cubic facets with preferential (100) orientation. The higher roughness factors (ECSA/geometric electrode area) of these nanostructured 
electrodes would lead to an elevated local $\mathrm{pH}$ close to the electrode surface, which has been shown to favor $\mathrm{C}_{2} \mathrm{H}_{4}$ over $\mathrm{CH}_{4}$, so this is another likely reason for the altered product distribution compared to more planar electrodes.

\section{Other $\mathrm{Cu}_{2} \mathrm{O}$ film-derived electrodes}

A third class of oxide-derived materials are electrodes derived from copper(I)oxide films, prepared by electrodeposition or hydrothermal synthesis onto a substrate. ${ }^{116,129,216,330}$ A rough surface is again created after reduction of the oxide layer, although typically less rough than the calcined electrodes described above. Examples of such films of different thicknesses as-deposited and after reduction are shown in Figure 25, illustrating the change in morphology induced by removal of lattice oxygen. $\mathrm{CO}_{2} \mathrm{R}$ measurements on this class of materials resulted in suppression of methane and enhanced $\mathrm{C}_{2}$ product formation, with ethylene, ethane and ethanol being the main products. In the different studies, the effects of bulk crystal structure, film thickness and crystallite size were discussed, giving minor variations in product distribution. Interestingly, these methods for preparing oxide-derived, nanostructured electrodes overall yield similar results to the anodic treatments discussed above with regards to methane suppression and higher selectivity to $\mathrm{C}_{2+}$ products. On the other hand, some of the

calcined oxide-derived copper electrodes were highly selective towards $\mathrm{CO}$ and $\mathrm{HCOO}^{-}$at low overpotential, but exhibited modest hydrocarbon productions. A possible reason for this discrepancy could be the significantly higher roughness factors resulting from the thermally oxidized precursors as compared to the other methods of obtaining oxide-derived electrodes. A very high roughness factor leads to higher activities at lower overpotentials, where the $2 \mathrm{e}^{-}$products from $\mathrm{CO}_{2} \mathrm{R}, \mathrm{CO}$ and $\mathrm{HCOO}^{-}$, are preferred. This could also lead to these catalysts being mass-transport limited in a region where they would otherwise produce significant amounts of $>2 \mathrm{e}^{-}$hydrocarbon and/or oxygenates. 

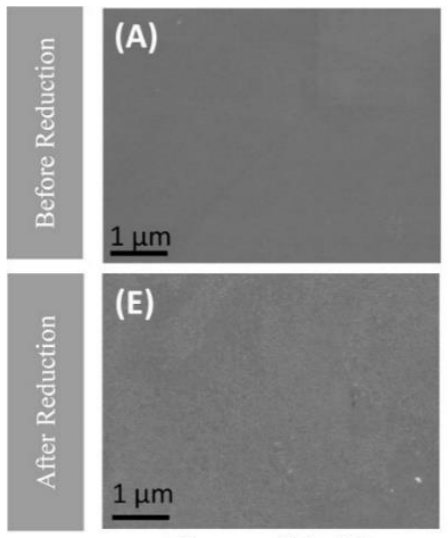

Electropolished $\mathrm{Cu}$
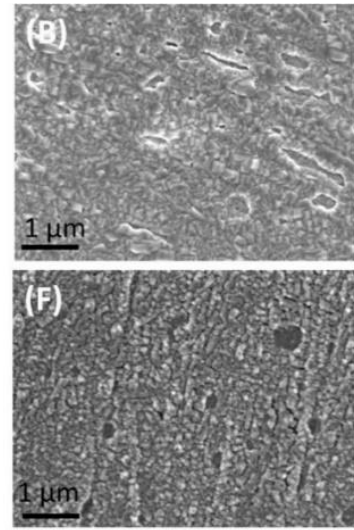

$0.2 \mu \mathrm{m} \mathrm{Cu}_{2} \mathrm{O}$ film
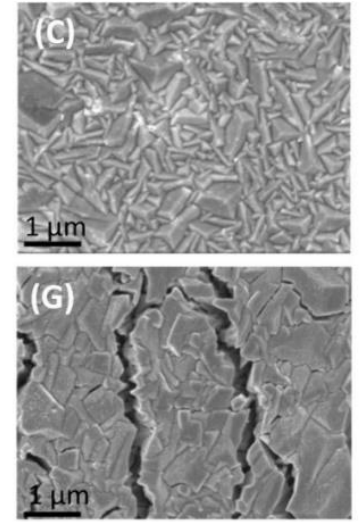

$1.7 \mu \mathrm{m} \mathrm{Cu}_{2} \mathrm{O}$ film
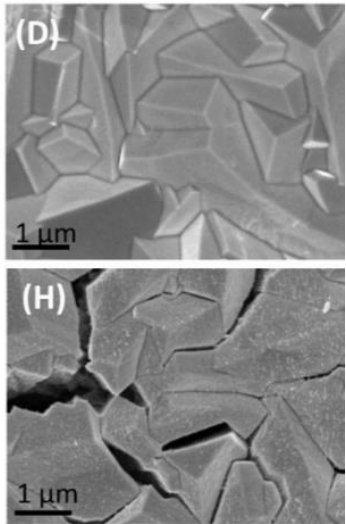

$8.8 \mu \mathrm{m} \mathrm{Cu}_{2} \mathrm{O}$ film

Figure 25: $\mathrm{SEM}$ micrographs of various $\mathrm{Cu}_{2} \mathrm{O}$ films prepared by electrodeposition. (a)-(d) Asdeposited and (e)-(h) after $\mathrm{CO}_{2} \mathrm{R}$ at $-0.99 \mathrm{~V} v$ s. RHE. Reprinted from Ren et al. ${ }^{129}$ Copyright (C) 2015, American Chemical Society.

\subsubsection{Non-Oxide-Derived Copper}

While it is important to note that a thin native oxide surface layer is formed on $\mathrm{Cu}$ whenever exposed to ambient air, here we would like to briefly discuss 'non-oxide-derived' copper catalysts. By this we mean to highlight several approaches other than those based on the intentional formation and reduction of a thick copper oxide layer on the surface that have been utilized for preparing 3-D copper electrodes, such as sputter deposition, electrodeposition, and dealloying. ${ }^{131,331-336}$ Interestingly, similar trends as for the anodically treated and other $\mathrm{Cu}_{2} \mathrm{O}$-derived catalysts discussed previously were observed in all of these studies. Increased $\mathrm{C}_{2+}$ and lower $\mathrm{C}_{1}$ product selectivity was reported compared to polycrystalline $\mathrm{Cu}$, and specifically, ethylene and sometimes ethanol formation reached significant selectivity at lower overpotentials. It seems that many approaches can lead to significantly increased $\mathrm{C}_{2+}$ over $\mathrm{C}_{1}$ product selectivity from $\mathrm{CO}_{2} \mathrm{R}$ compared to polycrystalline $\mathrm{Cu}$. The independence of the resulting product distribution on the preparation method suggests that the reason for the improved selectivity is related to the broader impacts of nanostructuring (such as increased surface area), and not necessarily an oxide precursor.

This hypothesis was supported by two recent works where multiple different nanostructured $\mathrm{Cu}$ electrodes were prepared and tested for $\mathrm{CO}_{2} \mathrm{R}$. Lum et al. synthesized four different types of oxidederived $\mathrm{Cu}$ electrodes, spanning the three different groups discussed above, and studied their $\mathrm{C}_{2+}$ product selectivity from $\mathrm{CO}_{2} \mathrm{R}$ between -0.7 and $-1.0 \mathrm{~V}$ vs. RHE. ${ }^{98}$ The varying ECSA of the different 
catalysts enabled determination of an ideal roughness to optimize $\mathrm{C}_{2+}$ product selectivity at the two extreme potentials, as shown in Figure 26. The authors attributed this optimum to a compromise between elevated local $\mathrm{pH}$, which has been shown to enhance $\mathrm{C}_{2+}$ product formation, and sufficient transport of $\mathrm{CO}_{2}$ to the active sites. This hypothesis was corroborated by mass transport modeling. ${ }^{98,99}$ Ren et al. utilized a similar approach, where they prepared four different types nanostructured electrodes by electrodeposition; three were oxide-derived and one was not. ${ }^{290}$ The trends that they observed could all be related to the surface area of the electrode, regardless of whether the material was derived from an oxidized precursor or not. The authors concluded that in order to obtain selectivity towards certain products, the material should be designed to have a limiting current density in a potential region where $\mathrm{Cu}$ is selective for a certain product. ${ }^{290}$ If, for instance, $2 \mathrm{e}^{-}$products are desired, the electrodes should exhibit very high roughness so that significant current can be achieved at low overpotential. As discussed previously, such electrodes reach mass transport limitations early, and therefore produce small amounts of $>2 \mathrm{e}^{-}$products. Thus, if the aim is to produce oxygenates and/or hydrocarbons, intermediate to low roughness is desirable, as was also illustrated by Lum et al. in Figure $26 .{ }^{98}$ 

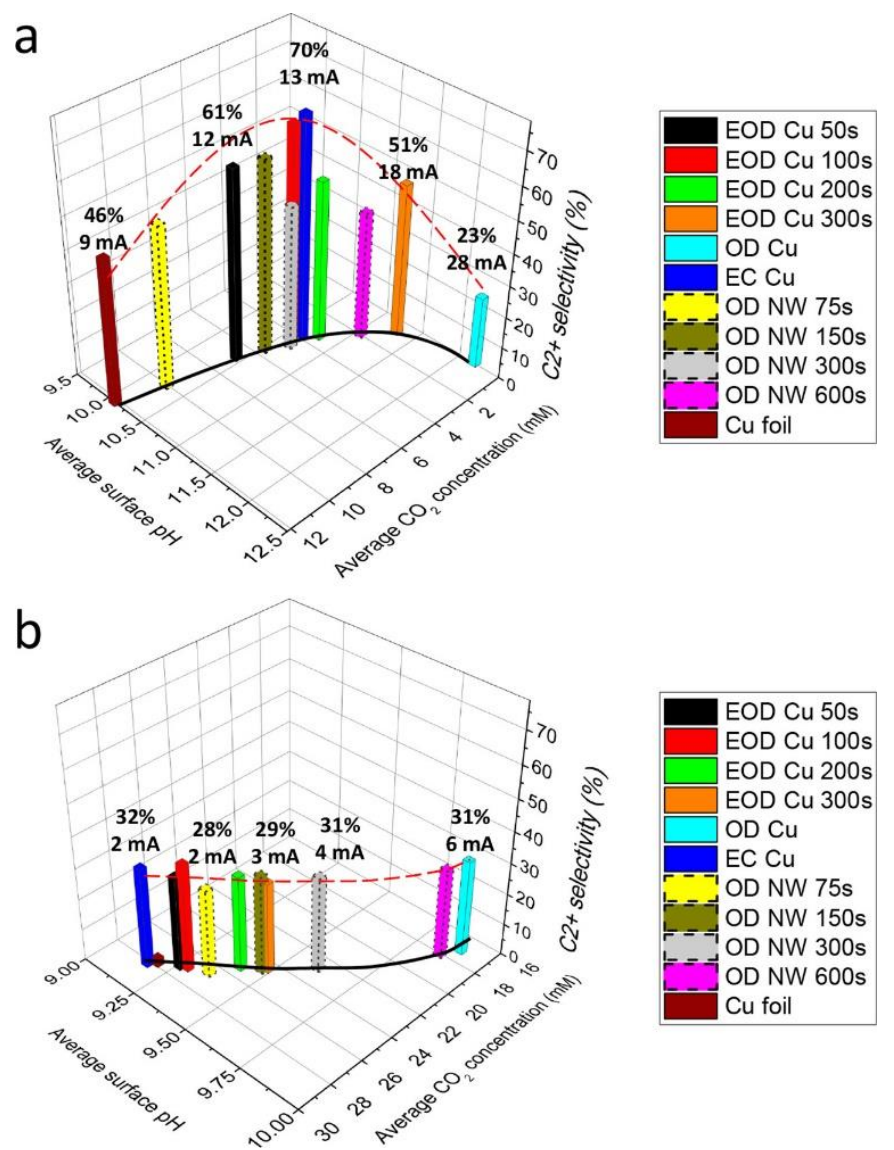

Figure 26: $\mathrm{C}_{2+}$ product selectivity from $\mathrm{CO} 2$ reduction on various oxide-derived $\mathrm{Cu}$ electrodes in 0.1 $\mathrm{M} \mathrm{KOH}$ at a) $-1.0 \mathrm{~V}$ and b) $-0.7 \mathrm{~V}$. Reprinted from Lum et al. ${ }^{98}$ Copyright $\odot$ 2017 , American Chemical Society.

As previously mentioned, many nanostructured electrodes are ill-defined, making it difficult to deconvolute the influence of different possible reasons for changes in behavior. Effects that can play a role include changes in mass transport and increase in local $\mathrm{pH}$, re-adsorption and further reduction of products, and higher occurrence of undercoordinated sites. One approach to try to deconvolute surface area effects from other effects is to prepare electrodes with ordered pore structure. Yang et al. prepared electrodes of more well-defined porosity by sputtering $\mathrm{Cu}$ onto a structured template. ${ }^{332}$ This resulted in a $\mathrm{Cu}$ film with cylindrical pores of varying width and depth, as displayed in Figure 27. They found that pores of $30 \mathrm{~nm}$ diameter and $40 \mathrm{~nm}$ (Figure 27a) depth yield mainly $\mathrm{C}_{2} \mathrm{H}_{4}$ at $-1.3 \mathrm{~V} v$ s. RHE, while $\mathrm{CH}_{4}$ is the main $\mathrm{CO}_{2} \mathrm{R}$ product on polycrystalline electrodes. Deeper pores (Figure 27b) led to enhanced $\mathrm{C}_{2} \mathrm{H}_{6}$ selectivity, while wider pores (Figure 27c) gave product distributions more similar to polycrystalline $\mathrm{Cu}$. The authors attributed the increase in $\mathrm{C}_{2}$ production for the electrodes with narrow 
pores to an increased local $\mathrm{pH}$. The change from ethylene to ethane with deeper pores could be caused by re-adsorption and further reduction of the products due to the longer retention time in the catalyst layer. Another similar study was performed by Song et al. on ordered, mesostructured $\mathrm{Cu}$ inverse-opal electrodes. ${ }^{337}$ As expected from the above arguments, they observed improved $\mathrm{C}_{2+}$ selectivity with increasing thickness of the porous $\mathrm{Cu}$ layer. This type of systematic studies is important in order to help identify of the factors that lead to improved performance of 3-D electrodes. We therefore suggest this as an area of increased attention in the future.
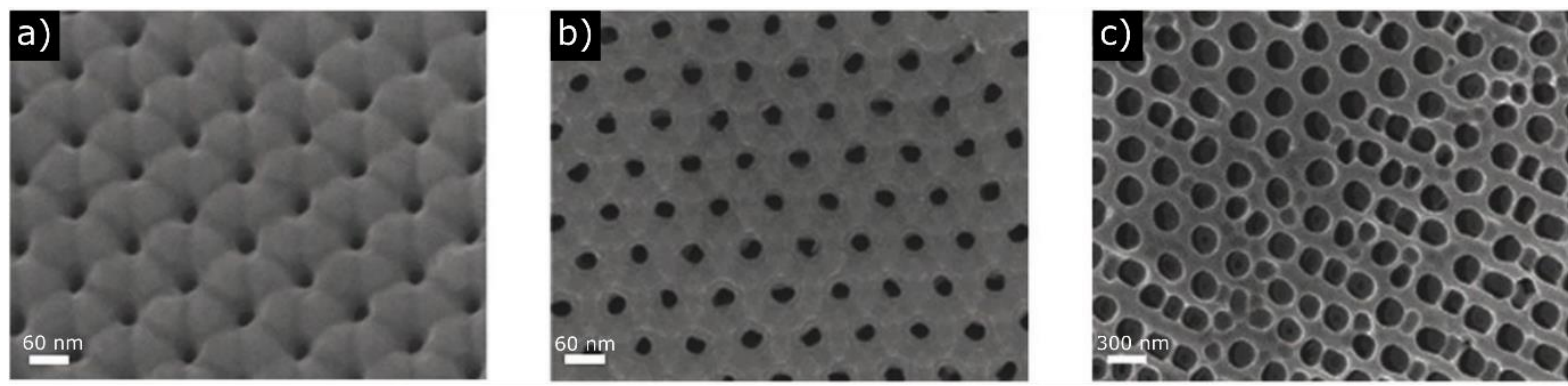

Figure 27: SEM images of mesoporous $\mathrm{Cu}$ films with holes of different dimensions. a) $30 \mathrm{~nm}$ width and $40 \mathrm{~nm}$ depth, b) $30 \mathrm{~nm}$ width and $70 \mathrm{~nm}$ depth and c) $300 \mathrm{~nm}$ width and $40 \mathrm{~nm}$ depth. Reprinted with permission from Yang et al. ${ }^{332}$ Copyright @ 2016, John Wiley and Sons.

\subsubsection{The Role of Oxygen in $\mathrm{CO}_{2} \mathrm{R}$ Electrocatalysis}

The role of oxygen in $\mathrm{CO}_{2}$ electroreduction has emerged as a topic of acute discussion within the field in the last few years. The topic has, however, been debated for decades already, starting with the previously mentioned report by Frese in the early 1990's. ${ }^{320}$ As described earlier, oxide-derived nanostructured materials have been shown to yield interesting selectivity effects for $\mathrm{CO}_{2} \mathrm{R}$ and COR. An important question is whether this is a result of the nanostructure, or oxygen from the oxidized precursor remaining close to the surface under reaction conditions. Intuitively, one would expect any oxides to be reduced under the $\mathrm{CO}_{2} \mathrm{R}$ conditions, due to the strong thermodynamic driving force from the negative potentials applied (see Table 5). Recent DFT calculations indeed suggested that reduction of $\mathrm{Cu}$ oxides is more favorable than $\mathrm{CO}_{2} \mathrm{R}$ at the relevant potentials. ${ }^{136}$ However, a number of reports 
explain that some amount of the initial oxide can be stabilized and affects catalysis. In the following, we will discuss the relevant studies that argue the two points of view.

Table 5: Equilibrium potentials for the reduction various copper oxide species. Calculated with Thermochemistry data from NIST as described in Section 1 of the SI.

\begin{tabular}{|c|c|}
\hline Reaction & $\mathbf{E}^{0} / \mathbf{V}$ vs. RHE \\
\hline $\mathrm{CuO}+2\left(\mathrm{H}^{+}+\mathrm{e}^{-}\right) \leftarrow \rightarrow \mathrm{Cu}+\mathrm{H}_{2} \mathrm{O}$ & 0.56 \\
$2 \mathrm{CuO}+2\left(\mathrm{H}^{+}+\mathrm{e}^{-}\right) \leftarrow \rightarrow \mathrm{Cu}_{2} \mathrm{O}+\mathrm{H}_{2} \mathrm{O}$ & 0.66 \\
$\mathrm{Cu}_{2} \mathrm{O}+2\left(\mathrm{H}^{+}+\mathrm{e}^{-}\right) \leftarrow \rightarrow 2 \mathrm{Cu}+\mathrm{H}_{2} \mathrm{O}$ & 0.46 \\
$\mathrm{Cu}(\mathrm{OH})_{2}+2\left(\mathrm{H}^{+}+\mathrm{e}^{-}\right) \leftarrow \rightarrow \mathrm{Cu}+2 \mathrm{H}_{2} \mathrm{O}$ & 0.53 \\
$\mathrm{Cu}_{2} \mathrm{CO}_{3}(\mathrm{OH})_{2}+4\left(\mathrm{H}^{+}+\mathrm{e}^{-}\right) \leftarrow \rightarrow 2 \mathrm{Cu}+3 \mathrm{H}_{2} \mathrm{O}+\mathrm{CO}_{2}$ & 0.55 \\
\hline
\end{tabular}

DFT calculations have been applied both to study whether or not oxygen can be stable under reaction conditions and how it could potentially affect catalysis. Favaro et al. studied the effect of subsurface oxygen on the physisorption of $\mathrm{CO}_{2}$ onto a $\mathrm{Cu}$ surface in a gaseous environment. Using DFT and ambient pressure X-ray photoelectron spectroscopy (APXPS), they showed that subsurface oxygen was critical to achieve stable adsorption of $\mathrm{CO}_{2}$ onto the surface. ${ }^{338}$ Similarly, Kim et al. used XPS depth profiling before and after $\mathrm{CO}_{2} \mathrm{R}$ as evidence that a copper oxide phase catalyzes the $\mathrm{CO}_{2} \mathrm{R} .{ }^{330}$ There remains a gap to be addressed, however, between these $e x$-situ results and true $\mathrm{CO}_{2} \mathrm{R}$ conditions, since adding electrolyte to the system and applying negative polarization of the cathode are likely to have a significant influence. Liu et al. suggested that subsurface oxygen close to the surface can actually be stabilized by the disordered near-surface structure and roughness of oxide-derived copper. ${ }^{339}$ Furthermore, they argued that near-surface oxygen can enhance the $\mathrm{CO}$ binding strength by withdrawing electron density from surface atoms and reduce the $\sigma$ repulsion between $\mathrm{CO}$ and surface $\mathrm{Cu}$ atoms. Other calculations showed that only oxygen in the topmost two subsurface layers can have a non-negligible influence on the reactivity of surface atoms, but that the barrier for diffusion of these oxygen atoms are too low for them to be stable under reaction conditions. ${ }^{340,341}$

A number of further $e x$-situ studies have been carried out showing that oxygen is present after $\mathrm{CO}_{2} \mathrm{R}^{320,330,342-345}$ These groups applied techniques such as X-ray photoelectron spectroscopy, Auger 
electron spectroscopy and TEM, showing copper oxides present throughout the catalyst. However, exsitu studies after transfer through air are problematic when studying copper, because of the rapid reoxidation upon exposure to $\mathrm{O}_{2} \cdot{ }^{346,347}$ As discussed previously, reduction of $\mathrm{Cu}$ oxide films before or during $\mathrm{CO}_{2} \mathrm{R}$ leads to formation of nanostructured surfaces. The high abundance of defects on such surfaces ensures even faster oxidation in ambient air after $\mathrm{CO}_{2} \mathrm{R},{ }^{347}$ making it even harder to draw conclusions about their oxidation state under reaction conditions. Recently, Cavalco et al. kept their electrodes under an inert gas atmosphere when transferring them to characterization by TEM, and still saw significant amounts of oxygen. ${ }^{344}$ It is, however, difficult to ensure that absolutely no oxygen reaches the sample at any point in the process. One way to get around oxygen contamination when applying ex-situ techniques is using isotopically labelled oxygen. Lum et al. carried out depth profiling of oxide-derived $\mathrm{Cu}$ that had been oxidized anodically in $\mathrm{H}_{2}{ }^{18} \mathrm{O} \cdot{ }^{347}$ They saw that after only 10 minutes, less than $1 \%$ of the original content of ${ }^{18} \mathrm{O}$ was remaining, similarly to ${ }^{16} \mathrm{O}$ control samples, as shown in Figure 28. This suggests that at least a large fraction of the initial oxide is reduced under reaction conditions. Some recent studies have prepared $\mathrm{Cu}$ materials with other dopants than oxygen, such as sulfur ${ }^{144,348}$ and boron. ${ }^{349}$ This way materials can be prepared that are more resistant against reduction than those prepared with oxygen, while still retaining some of the effects that are thought to occur when $\mathrm{O}$ is present close to the surface. However, more work is needed to evaluate if this is actually a viable approach for future materials design. 
a

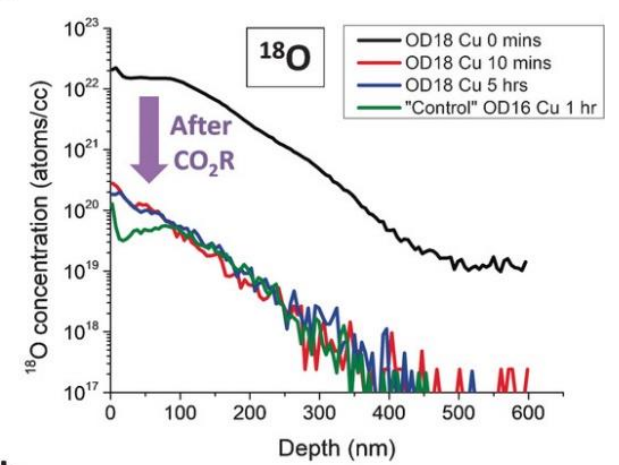

b

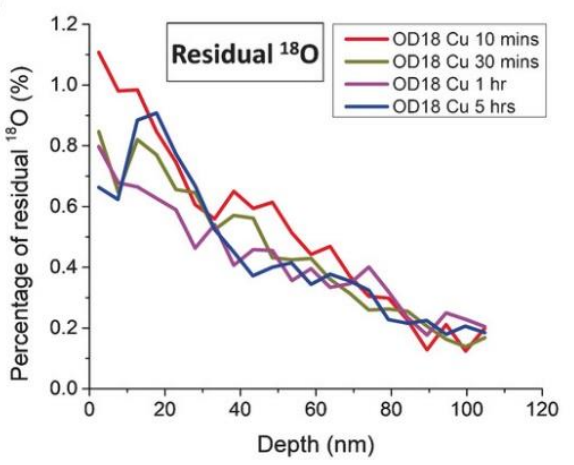

C

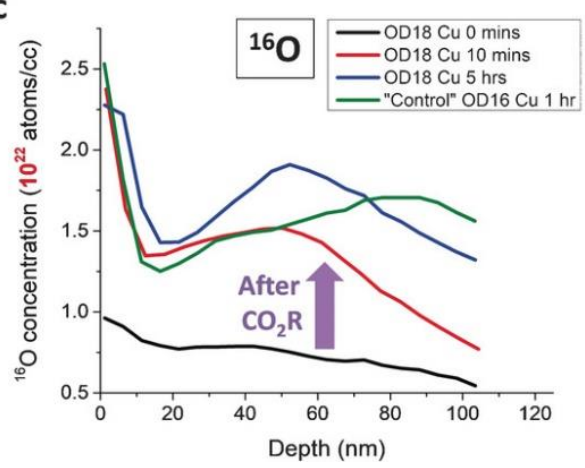

Figure 28: a) ${ }^{18} \mathrm{O}$ and c) ${ }^{16} \mathrm{O}$ content of $\mathrm{OD} \mathrm{Cu}$ catalysts with different $\mathrm{CO}_{2} \mathrm{R}$ durations measured by SIMS. b) The percentage of ${ }^{18} \mathrm{O}$ that remains in $\mathrm{OD} 18 \mathrm{Cu}$ after $\mathrm{CO}_{2} \mathrm{R}$. Reprinted with permission from Lum and Ager. ${ }^{347}$ Copyright $\odot$ 2018, John Wiley and Sons.

Because of the rapid re-oxidation of $\mathrm{Cu}$ in air, operando techniques are useful to study the oxidation state of these electrodes during $\mathrm{CO}_{2} \mathrm{R}$. A number of studies showed reduction of the oxidized precursor under reaction conditions using operando measurements such as X-ray absorption spectroscopy (XAS), ${ }^{125}$ Raman spectroscopy, ${ }^{96,129,130,136}$ and grazing incidence X-ray diffraction (GIXRD). ${ }^{177}$ Other operando studies suggest that oxygen is present under reaction conditions. ${ }^{126,250,350}$ However, there is uncertainty regarding these techniques as well. Catalysis happens at the surface, and only the reactivity of the topmost layers of atoms matter. As discussed above, the presence of different species, such as oxygen, close to the surface can alter this reactivity and affect catalysis, but they need 
to be within a few atomic layers underneath the surface. ${ }^{339,341}$ Most of the operando techniques employed thus far cannot clearly distinguish whether the oxygen is at or close to the surface, or deeper in the bulk where it will not affect catalysis. On the other hand, even small amounts of oxygen present at or close to the surface might affect reactivity, and most of the techniques would not be able to resolve such minute amounts of oxygen present at the surface.

As a general note, it is very challenging to design an experiment that conclusively determines the active site of a catalyst under reaction conditions. However, until evidence that oxygen species are stable close to the surface where they can influence catalysis is presented, it is the opinion of the authors that this is unlikely given the strong thermodynamic driving force for reduction. As discussed above, the fact that $\mathrm{CO}_{2} \mathrm{R}$ on oxide-derived $\mathrm{Cu}$ materials lead to a similar product distribution as non-oxidederived $\mathrm{Cu}$ further indicates that it is the nanostructure, and not the oxidation state of the precursor material, that gives the altered $\mathrm{CO}_{2} \mathrm{R}$ performance.

\subsubsection{Activity Comparisons}

Throughout Section 6.2, we have grouped the different 3-D materials into two main categories; oxide-derived and non-oxide-derived catalysts. Although a number of different approaches to nanostructuring have been applied in the literature, which have yielded electrodes with highly diverse structures, there are many similarities in their performance for $\mathrm{CO}_{2} \mathrm{R}$. Importantly, studies on 3-D electrodes carried out at low overpotentials report improved selectivity towards carbon monoxide and/or formate compared to planar copper foils. At more negative potentials, some or full suppression of methane formation and enhanced selectivity towards ethylene and other $\mathrm{C}_{2+}$ products has been reported.

Nanostructuring gives electrodes with increased roughness factors, varying from 2 to 850 in the studies discussed herein. This results in increased current density when normalized by geometric surface area. In Figure 29a, geometric $\mathrm{CO}_{2} \mathrm{R}$ current densities for many of the studies discussed throughout this section are shown, compared to data obtained on planar, polycrystalline $\mathrm{Cu}$ by Kuhl et al. ${ }^{66}$ and Hori et al. ${ }^{85}$ In this plot, all the nanostructured materials exhibit higher geometric activity at a given potential than polycrystalline copper, when not under mass transport limited contitions. 
This does not, however, reflect the intrinsic activity of the material, as discussed in Section 2.1. Therefore, it is instructive to also compare the specific activity of the electrode, as shown in Figure $\mathbf{2 9 b}$, where the activities are normalized to ECSA. Here we see that there is little or no difference in the total intrinsic $\mathrm{CO}_{2} \mathrm{R}$ activity among the different types of nanostructured electrodes. Furthermore, the intrinsic activity of these various $\mathrm{Cu}$ nanostructures is similar to that of planar, polycrystalline $\mathrm{Cu}$. The similar intrinsic activity of all $\mathrm{Cu}$ electrodes is underlined by the Tafel-like behavior observed in Figure 29b, prior to diffusion limited conditions. This explains why high roughness factor materials tend to exhibit earlier onset; higher roughness electrodes yield higher overall current densities, which allows for the detection of products at lower overpotential, even at low turnover frequencies per surface site. The magnitude of the diffusion limited current density also scales with surface roughness, with high surface area materials reaching mass transport limitation at lower overpotential. These findings strongly suggest that nanostructuring does not alter the intrinsic activity of copper in any significant manner, perhaps within a factor of 2-4, for any synthesis method used. Furthermore, it does not seem that intrinsic $\mathrm{CO}_{2} \mathrm{R}$ activity is higher for oxide-derived materials (studies [C-J] in Figure 29), as compared to non-oxide-derived electrodes such as polycrystalline $\mathrm{Cu}$ (studies $[\mathrm{A}, \mathrm{B}]$ ) and mesostructured $\mathrm{Cu}$ (study $[\mathrm{K}]$ ). This further corroborates the notion that the precursor material oxidation state is not a crucial factor for $\mathrm{CO}_{2} \mathrm{R}$ performance, ${ }^{98,290}$ and that any subsurface oxygen that may or may not be present based on different preparations of $\mathrm{Cu}$ has little or no influence on intrinsic $\mathrm{CO}_{2} \mathrm{R}$ activity. 


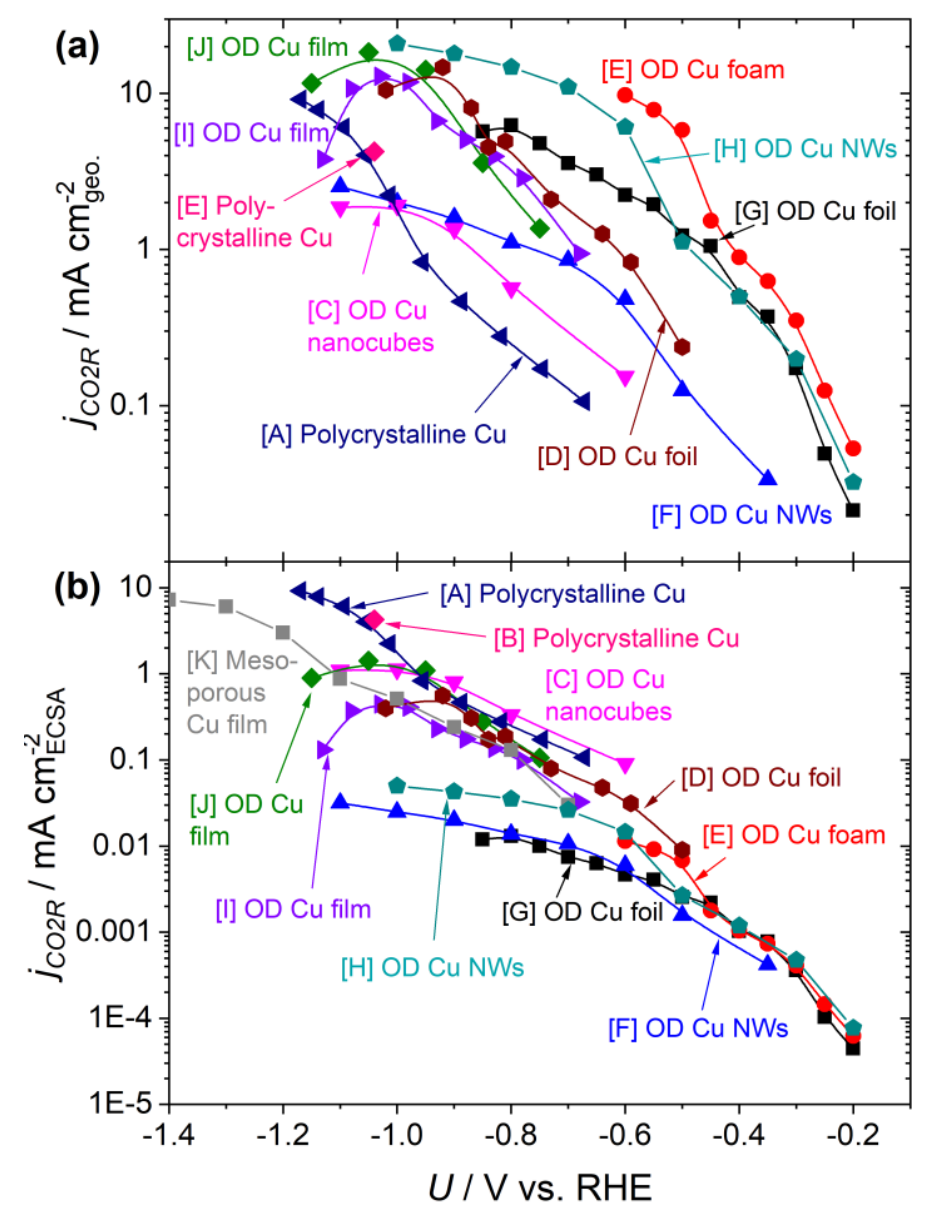

Figure 29: Comparison of $\mathrm{CO}_{2} \mathrm{R}$ activity for various nanostructured $\mathrm{Cu}$ electrocatalysts with polycrystalline $\mathrm{Cu}$. (a) $\mathrm{CO}_{2} \mathrm{R}$ partial current density normalized to geometric surface area. (b) $\mathrm{CO}_{2} \mathrm{R}$ partial current density normalized to electrochemical surface area (ECSA). The data were obtained from the following studies: [A] Kuhl et al. ${ }^{66}$; [B] Hori et al..$^{85}$; [C] Kwon et al. ${ }^{96}$; [D] Mistry et al. ${ }^{126}$; [E] Min et al..$^{94} ;[\mathrm{F}]$ Ma et al. ${ }^{93,321} ;[\mathrm{G}] \mathrm{Li}$ et al. ${ }^{90} ;[\mathrm{H}]$ Raciti et al. ${ }^{92} ;[\mathrm{I}]$ Handoko et al. ${ }^{216} ;[\mathrm{J}]$ Ren et al. ${ }^{328} ;[\mathrm{K}]$ Yang et al. ${ }^{332}$. Studies $[\mathrm{A}, \mathrm{B}]$ are polycrystalline $\mathrm{Cu},[\mathrm{C}-\mathrm{J}]$ are oxide-derived, nanostructured $\mathrm{Cu}$ and $[\mathrm{K}]$ is non-oxide-derived nanostructured $\mathrm{Cu}$.

Although the ECSA-normalized activity is similar for the various types of nanostructured electrodes, there are differences in the product distribution. First, as mentioned previously, mass transport conditions generally have a large influence on the product distribution obtained from a catalyst material. When approaching mass transport limited conditions, the local $\mathrm{pH}$ close to the electrode surface increases. Since electrodes with high surface area reach mass transport limitation at relatively low overpotentials, improved $\mathrm{C}_{2+}$ product selectivity can be observed under these conditions. However, as significant mass transport limitations are reached, too little $\mathrm{CO}_{2}$ reaches the electrode surface, and $\mathrm{H}_{2}$ evolution takes over. This is a likely reason for calcined oxide-derived $\mathrm{Cu}$ foils exhibiting a significant drop in $\mathrm{CO}_{2} \mathrm{R}$ selectivity at potentials more negative than $-0.6 \mathrm{~V}$ vs. RHE, and low 
hydrocarbon formation in general. The very high roughness factors cause the bulk of the non-masstransport-limited activity to be in a low overpotential region where $\mathrm{CO}_{2} \mathrm{R}$ to $\mathrm{CO}$ and $\mathrm{HCOO}^{-}$is favored; meanwhile, these systems favored $\mathrm{C}_{2+}$ oxygenates at low overpotentials when doing COR (since the overpotential to reduce $\mathrm{CO}_{2}$ to $\mathrm{CO}$ is not a limiting factor), which can be understood by the potentialdependent selectivity trends shown in Figure 24. Thus, a key finding here is that the roughness factor of the electrode in effect shifts the potential window where kinetically-limited $\mathrm{CO}_{2} \mathrm{R}$ can take place, which has a very large impact on the product selectivity.

Electrode roughness is not the only important parameter though; morphological features such as pore size and shape can also strongly influence product distribution, as well as the retention time of products and intermediates in the catalyst structure. A good illustration of this is that studies investigating catalyst layer thickness or pore depth generally find that thicker nanostructures lead to more ethane on behalf of ethylene. Ethane is generally not observed on planar electrodes, and can likely be attributed to re-adsorption and further reduction of ethylene. Although clear trends are outlined above, more work is still needed to improve our understanding of which effects drive selectivity to different products, and eventually give us the possibility to steer $\mathrm{CO}_{2} \mathrm{R}$ towards desirable chemicals. Furthermore, since current densities of $100 \mathrm{~mA} \mathrm{~cm}{ }^{-2}$ or higher seems to be a minimum for commercialization to be realistic, ${ }^{60}$ more work needs to be done to enhance mass transport of $\mathrm{CO}_{2}$ to achieve higher reaction rates. A key strategy towards that end will be discussed subsequently.

\subsection{Vapor-Fed Systems}

All of the catalysts described thus far have been tested using conventional aqueous-phase $\mathrm{CO}_{2} \mathrm{R}$ reactors. While these have clearly been useful for gaining fundamental insights, the poor solubility of $\mathrm{CO}_{2}$ in aqueous electrolytes $(\sim 34 \mathrm{mM})$, along with the acid/base buffer equilibria $\left(\mathrm{CO}_{2} / \mathrm{HCO}_{3}-\mathrm{CO}_{3}^{-2}\right)$ described earlier lead to significant challenges in achieving high $\mathrm{CO}_{2} \mathrm{R}$ reaction rates. Vapor-fed systems can overcome these practical limitations by delivering gas phase $\mathrm{CO}_{2}$ directly to the catalyst surface. Indeed, some of the best performing $\mathrm{Cu}$ catalyst systems reported today in terms of Faradaic efficiency, energy conversion efficiency, and stability use vapor-fed reactors. ${ }^{127,132,293,331,348,351-354} \mathrm{We}$ 
therefore wanted to take this opportunity to briefly discuss these reactor configurations and highlight promising developments in this area. For more information, we direct the readers to a recent perspective article that details the progress, challenges, and opportunities towards the development of practical $\mathrm{CO}_{2}$ electrolyzers, ${ }^{355}$ as well as a recent review on the same topic. ${ }^{356}$

In commercial electrocatalytic systems, such as fuel cells and water electrolyzers, a gas diffusion layer is typically employed as a support material in order to maximize transport of reactants to the catalyst surface. These layers are comprised of open, three-dimensional structures of relatively inert materials, such as carbon. Together with the porous catalyst, this forms what is known as a gas diffusion electrode (GDE). A GDE can be used with either a liquid electrolyte or a solid-state polymer electrolyte membrane. A membrane electrode assembly (MEA) refers to the latter case, whereby an assembled stack is composed of a polymer electrolyte membrane sandwiched between two GDEs. Here, ionomers are used to extend the electrolyte membrane material into the GDEs to provide intimate contact with the catalyst and ensure efficient transport of ions to/from the surface. This is important because the relevant reactions can only take place at triple-phase boundaries, which are points where the gaseous reactant, electron-conduction catalyst, and ion-conducting polymer or liquid electrolyte meet. Vapor-fed systems are thus inherently more complex than aqueous-phase systems due to the multitude of heterogeneous interfaces across a range of length scales (Figure 30) and will present many challenges in optimizing $\mathrm{CO}_{2} \mathrm{R}$ activity and selectivity.

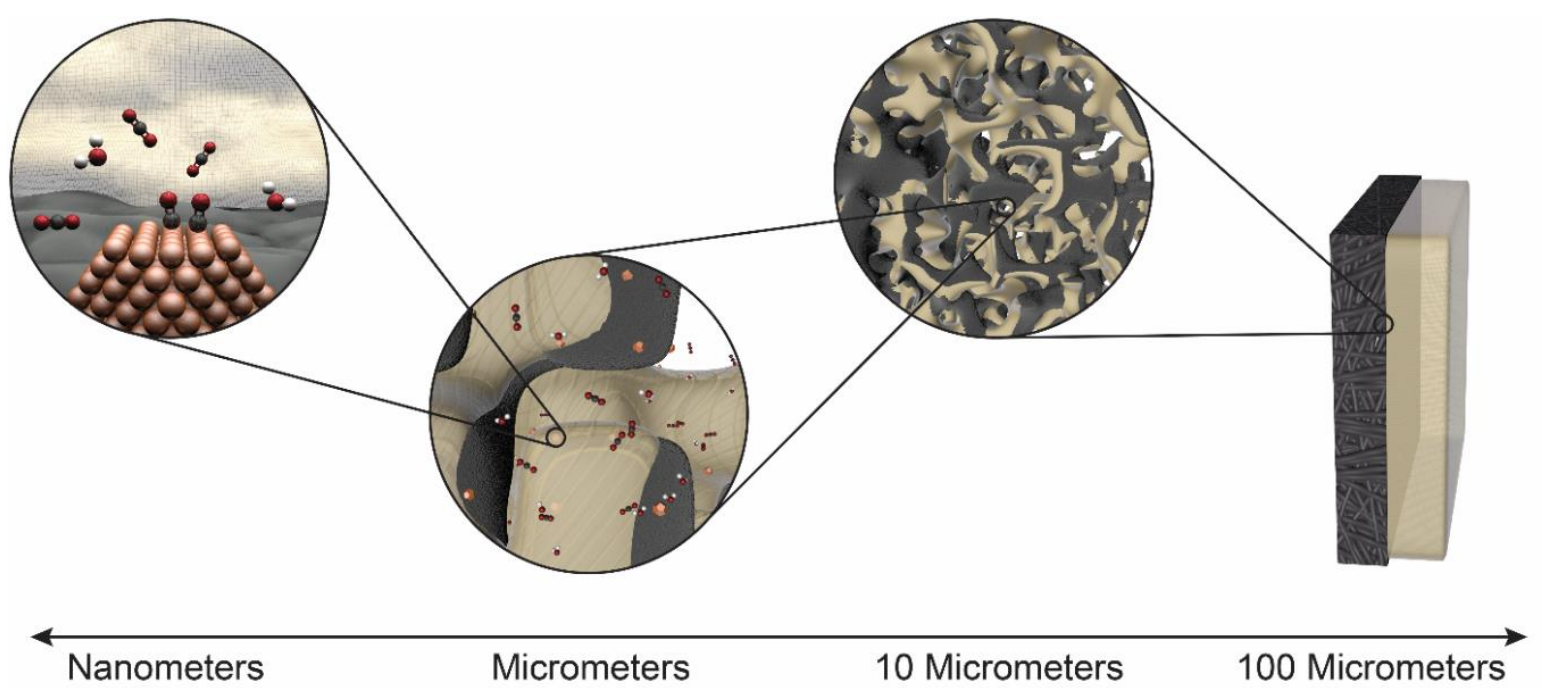

Figure 30: Schematic of a 3-dimensional GDE depicting the multiple length scales where phenomena are occurring during electrochemical $\mathrm{CO}_{2} \mathrm{R}$. Reprinted from Higgins et al. ${ }^{355}$ Copyright (C) 2018, American Chemical Society, 
While still in its infancy, research in these areas have already demonstrated promising activity and selectivity improvements compared to conventional aqueous-phase reactors. ${ }^{127,132,293,331,348,351-353}$ For example, various vapor-fed reactors using $\mathrm{Cu}$-based GDEs have achieved high $\mathrm{CO}_{2} \mathrm{R}$ geometric partial current densities to ethylene of around $150-473 \mathrm{~mA} / \mathrm{cm}^{2},{ }^{127,132,293,331,352,353}$ and to ethanol of around $40-108 \mathrm{~mA} / \mathrm{cm}^{2} .^{132,293,348,352,353}$ As discussed in Subsection 3.3.1, alkaline electrolytes can improve selectivities and energy efficiencies to $\mathrm{C}_{2+}$ products. This was illustrated in a recent report where researchers tested various electrolytes including $\mathrm{KOH}, \mathrm{KHCO}_{3}, \mathrm{KCl}$, and $\mathrm{K}_{2} \mathrm{SO}_{4}$ in their microfluidic $\mathrm{CO}_{2}$ flow cell electrolyzer and found that an optimal production of $\mathrm{C}_{2+}$ products $(411$ $\mathrm{mA} / \mathrm{cm}^{2}$ at $-0.67 \mathrm{~V} v s$. RHE, corresponding to $62 \%$ Faradaic efficiency) could be achieved in $\mathrm{KOH} .{ }^{353}$ Another study employed a range of strongly basic conditions (1-10 M KOH) and could achieve a partial current density for $\mathrm{C}_{2+}$ products as high as $608 \mathrm{~mA} / \mathrm{cm}^{2}$ at $-0.67 \mathrm{~V} v$ s. RHE (corresponding to $81 \%$ Faradaic efficiency). ${ }^{352}$ These carbon-based GDEs were unfortunately found to degrade at such high reaction rates, but the stability was improved by incorporating a polymer layer to help prevent flooding. In this way, Sargent and coworkers were able to achieve $\sim 70 \%$ Faradaic efficiency for $\mathrm{CO}_{2} \mathrm{R}$ to $\mathrm{C}_{2} \mathrm{H}_{4}$ at $-0.55 \mathrm{~V}$ vs. RHE for 150 hours in $7 \mathrm{M} \mathrm{KOH}$ (although the current slowly declined from 100 to 75 $\left.\mathrm{mA} / \mathrm{cm}^{2}\right){ }^{352}$ It is worth noting that for sustained $\mathrm{CO}_{2} \mathrm{R}$ under alkaline conditions, reactor systems will have to be carefully engineered to avoid neutralization of the electrolyte via $\mathrm{CO}_{2}$ conversion to bicarbonate; otherwise, regeneration of the $\mathrm{KOH}$ will add to the costs and detract from the overall energy balance of the system. This could also be one advantage of performing COR instead of $\mathrm{CO}_{2} \mathrm{R}$ in such a device.

The studies discussed above demonstrate the promise of using vapor-phase $\mathrm{CO}_{2}$ reactants in overcoming intrinsic limitations of aqueous-based systems, but there is still much work ahead in further developing and optimizing these practical device architectures for commercial application. The choice of electrolyte, catalyst, and GDE fabrication process will all have a significant impact on device performance and must ideally be designed to optimize transport of $\mathrm{CO}_{2}$, products, electrons, and ions simultaneously. There is thus a large phase space to explore in tuning relevant properties of the gas diffusion layer(s), such as porosity, hydrophobicity, and thickness. Well-controlled studies that aim to 
understand the effects of nanoparticle size, shape, and loading, as well as catalyst-support and catalystionomer interactions will also be essential. Translating this and other fundamental knowledge gained from probing catalyst morphology and electrolyte effects in aqueous-phase reactors to these complex vapor-fed systems will aid in their advancement. Furthermore, since $\mathrm{CO}_{2} \mathrm{R}$ in these 3-D architectures involves many processes over a range of length and time scales, it will become increasingly important to couple experimental and theoretical investigations. Continued development of microkinetic and 3-D transport modeling methods are promising candidates in that regard.

\subsection{Summary}

Nanostructuring gives electrodes with increased roughness factors, varying from 2 to 850 in the studies discussed in this chapter, which results in increased current density when normalized by geometric area of the electrode. However, when normalized by the ECSA of the electrode, it is found that the intrinsic activity of $\mathrm{Cu}$ catalysts is similar among all the different forms of electrodes reported to date, whether nanostructured or planar, and irrespective of the particular nanostructure or synthesis route. This highlights the importance of data normalization for determining whether higher catalytic activity results from a higher average turnover frequency or simply from an increased number of active sites.

While nanostructuring does not appear to change the intrinsic activity of $\mathrm{Cu}$, interesting changes in product distribution have been observed. For example, many 3-D catalyst materials exhibit some or full $\mathrm{CH}_{4}$ suppression and increased $\mathrm{C}_{2+}$ product selectivity. This has largely been explained by mass transport modeling studies, which have shown that an ideal compromise between $\mathrm{pH}$ increase and $\mathrm{CO}_{2}$ supply at the electrode surface gives an optimal $\mathrm{C}_{2+}$ selectivity on rough electrodes. ${ }^{9899}$ More generally, since the number of active sites impacts the potential window where high reaction rates are achieved, the catalyst surface area has emerged as a key determinant of product selectivity. Other morphological factors such as preferential faceting, grain boundaries, and pore shape and size may also lead to changes in $\mathrm{CO}_{2} \mathrm{R}$ selectivity due to surface structure effects and/or re-adsorption and further reduction of products. 
Nanostructured electrodes are particularly relevant because of their potential compatibility with vapor-fed reactors, a promising technology platform capable of achieving industrially relevant current densities. However, substantial efforts will be needed in order to understand how $\mathrm{CO}_{2} \mathrm{R}$ catalysts behave in such a different environment than the conventional aqueous-phase, lab-scale reactors that are typically employed today. We feel that research in these directions should be prioritized in order to begin accelerating the development of practical devices that convert $\mathrm{CO}_{2}$ to high value fuels and chemicals.

\section{Copper Bimetallics}

Changing structural properties of the catalyst material (e.g., via hierarchical nanostructuring, particle size/support effects, etc.) is one key tactic to alter the catalytic performance, as discussed in the previous chapter. Another approach being employed to improve the activity, selectivity, and stability of $\mathrm{Cu}$ for $\mathrm{CO}_{2} \mathrm{R}$ is the preparation of bimetallic systems. Bimetallics can enhance performance compared to monometallic catalysts by a variety of mechanisms including electronic ligand and strain effects $^{357-362}$ and geometric ensemble effects. ${ }^{357-359,363,364}$ The electronic effects refer to a change in the electronic structure of the host material after addition of another element, which alters the binding strength of intermediate adsorbates on the surface according to the $d$-band model. ${ }^{360,365}$ Geometric ensemble effects refer to a change in the atomic arrangement of actives sites, which alters the way adsorbates interact with the surface and each other. In addition to simply changing the number or configuration of particular atoms in an ensemble, bifunctional active sites can also be created, where neighboring metals serve different catalytic roles. For example, it has been thought that bifunctional active sites for $\mathrm{CO}_{2} \mathrm{R}$ could be created using a metal with higher oxygen affinity, allowing $\mathrm{COOH}^{*}$ or $\mathrm{CHO}^{*}$ to be stabilized by interacting with the surface through both the carbon and oxygen atoms compared to $\mathrm{CO}^{*}$, which tends to bind surfaces through the carbon atom in an upright geometry. ${ }^{203,366}$ Additionally, isolated active sites can be formed that change the proximity of other adsorbates, which can impact the selectivity. ${ }^{367}$ 
There are a multitude of bulk systems that can be formed when two elements are mixed, including solid solutions, intermetallic compounds, and phase-separated systems. Details governing the formation of these bimetallics and their different properties are outside the scope of this review. Instead, we will focus on the resulting model systems that allow for the study of the aforementioned electronic and geometric effects. These include bulk alloys, surface alloys (alloy layer supported on a monometallic core), near surface alloys (alloy beneath one or a few monometallic layers), or guest metal modified surfaces (e.g., islands or particles on a monometallic host). In addition, there are bimetallic overlayers, either planar or nanoparticulate (core-shell) structures, which refer to a monometallic surface supported on either a different metal, a bulk alloy, or a (near) surface alloy. Due to the large number of possible bimetallic combinations, with regard to both the composition and structural arrangements, computational screening approaches have been used to aid in the search for improved $\mathrm{CO}_{2} \mathrm{R}$ catalysts. ${ }^{203,366-371}$ There have been a number of non-Cu-based bimetallics that have demonstrated synergistic activity or selectivity, primarily to $2 \mathrm{e}^{-}$products. ${ }^{372-377}$ This chapter will only focus on the experimental results obtained for $\mathrm{Cu}$-based bimetallic systems in aqueous solutions under ambient conditions.

As described in Subsection 1.2.2, early experimental work led by Hori and coworkers showed that on $\mathrm{Fe}, \mathrm{Ni}, \mathrm{Pt}$, and $\mathrm{Pd}$, the competing HER is primarily observed, on $\mathrm{Ag}$, $\mathrm{Au}$, and $\mathrm{Zn}$ the major product is $\mathrm{CO}$, and on $\mathrm{Cd}, \mathrm{Pb}, \mathrm{Hg}, \mathrm{Tl}, \mathrm{Sn}$, and $\mathrm{In}$ the major product is $\mathrm{HCOO}^{-1,85}$ The $\mathrm{Cu}$-based bimetallic combinations discussed in the following sections are thus organized according to these groupings by the dominant $\mathrm{CO}_{2} \mathrm{R}$ selectivity of the guest metals. First, we will describe the structural properties of the studied model catalysts and summarize the main experimental findings with respect to their catalytic activity and selectivity for $\mathrm{CO}_{2} \mathrm{R}$ compared to pure $\mathrm{Cu}$. Then, we will provide some overall perspectives on the benefits of using bimetallics, pitfalls to avoid when investigating these systems, and suggestions for future studies. 


\subsection{Copper and $\mathrm{H}_{2}$ Producing Guest Metals (Fe, Ni, Pt, Pd)}

Mainly $\mathrm{H}_{2}$ is formed during $\mathrm{CO}_{2} \mathrm{R}$ on monometallic electrodes of group 8-10 transition metals. ${ }^{1,85}$ Thus, $\mathrm{Cu}-\mathrm{M}$ bimetallic electrodes (where $\mathrm{M}=\mathrm{Fe}, \mathrm{Ni}, \mathrm{Pt}$ ) typically result in increased HER activity and selectivity compared to pure $\mathrm{Cu}$. This has been shown for a variety of model systems: $\mathrm{Cu}$

electrodes modified with $\mathrm{Fe}$, Ni, or Pt deposits, ${ }^{378,379} \mathrm{CuPt}$ alloy nanocubes and nanocrystals, ${ }^{380,381}$ and $\mathrm{Cu}$ overlayers on Pt electrodes. ${ }^{382,383}$ Further, trends have been observed whereby increased guest metal coverage on $\mathrm{Cu}$, increased $\mathrm{Pt}$ content in the nanoalloys, and decreased $\mathrm{Cu}$ overlayer thickness led to increased $\mathrm{H}_{2}$ activity and/or selectivity. In the case of the $\mathrm{Cu}$ overlayers, only after the $\mathrm{Cu}$ layer was sufficiently thick ( $>5 \mathrm{~nm}$ ) did the $\mathrm{H}_{2}$ formation return to bulk Cu-like values. ${ }^{383} \mathrm{In}$ a separate study, it was demonstrated using electrochemical scanning tunneling microscopy (ECSTM) that the presence of $\mathrm{CO}$ formed during $\mathrm{CO}_{2} \mathrm{R}$ destabilizes thinner $\mathrm{Cu}$ overlayers due to the very high binding affinity of $\mathrm{CO}$ on $\mathrm{Pt}^{382}$ This causes restructuring from a flat morphology into more complex three dimensional particles, while simultaneously exposing the underlying Pt substrate, as shown in Figure 31. ${ }^{382}$ Thus, the increased affinity for HER in the bimetallic systems likely results from increased exposure of these guest metals on the catalyst surface.
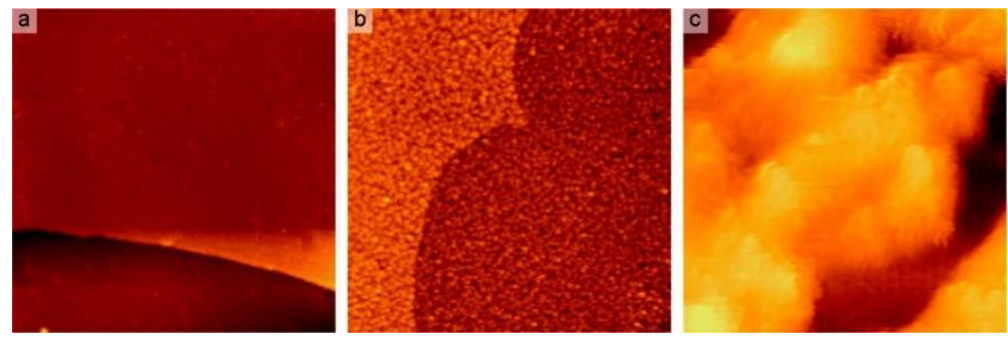

Figure 31: a) Atomically flat morphology of the Cu overlayer in a CO-free electrolyte $(499 \mathrm{~nm})^{2}, \mathrm{U}=$ $0.02 \mathrm{~V}, \mathrm{U}_{\mathrm{B}}=292 \mathrm{mV}, \mathrm{I}_{\mathrm{T}}=1 \mathrm{nA}$. b) Granular morphology of the Cu overlayer after exposure to a COsaturated electrolyte $(499 \mathrm{~nm})^{2}, \mathrm{U}=0.01 \mathrm{~V}, \mathrm{U}_{\mathrm{B}}=356 \mathrm{mV}, \mathrm{I}_{\mathrm{T}}=10 \mathrm{nA}$. c) Disordered Cu overlayer with $(2 \times 2)$-CO structure inside the vacancies $(13.6 \mathrm{~nm})^{2}, \mathrm{U}=0.01 \mathrm{~V}, \mathrm{U}_{\mathrm{B}}=356 \mathrm{mV}, \mathrm{I}_{\mathrm{T}}=10 \mathrm{nA}$. Reprinted from Varela et al. ${ }^{382}$ Copyright (C) 2013, American Chemical Society.

As one might expect, these systems also exhibit a concurrent decrease in overall $\mathrm{CO}_{2} \mathrm{R}$ activity and selectivity, and in general only mixtures of $\mathrm{H}_{2}, \mathrm{CO}, \mathrm{CH}_{4}$, and $\mathrm{C}_{2} \mathrm{H}_{4}$ were detected. Interestingly, with increasing $\mathrm{Ni}$ or $\mathrm{Fe}$ coverage on $\mathrm{Cu}$, Hori found a linear correlation between the HER selectivity and $\mathrm{CH}_{4} / \mathrm{C}_{2} \mathrm{H}_{4}\left(\mathrm{C}_{1} / \mathrm{C}_{2}\right)$ selectivity ratio. ${ }^{378}$ It is worth noting, however, that the increase in $\mathrm{C}_{1} / \mathrm{C}_{2}$ ratio 
was due to significant $\mathrm{C}_{2} \mathrm{H}_{4}$ inhibition rather than a boost in $\mathrm{CH}_{4}$ production. This trend extends across many of these other systems, where $\mathrm{C}_{2} \mathrm{H}_{4}$ was only detected in trace quantities ${ }^{379,382}$ or not at all..$^{380,381}$ Based on the mechanistic studies discussed earlier, this decreased $\mathrm{C}_{2} \mathrm{H}_{4}$ production may be attributed to increased $\mathrm{H}$ coverage on the surface, which can hinder C-C coupling. In the nanoalloys and guest metal modified $\mathrm{Cu}$ systems, this can also be due to a lack of adjacent $\mathrm{Cu}$ surface sites required for $\mathrm{CO}$ dimerization. However, for the thin $\mathrm{Cu}$ overlayer system, it was determined via ECSTM that even after sintering, the $\mathrm{Cu}$ ensembles would still be large enough to allow for C-C bond formation, but the $\mathrm{C}_{2} \mathrm{H}_{4}$ Faradaic efficiency did not exceed $0.6 \% .{ }^{382}$ Meanwhile, for thicker $\mathrm{Cu}$ overlayers $(>5 \mathrm{~nm}$ ), it was found that the ratio between $\mathrm{CH}_{4}$ and $\mathrm{C}_{2} \mathrm{H}_{4}$ could be tuned as a function of overlayer thickness and potential. ${ }^{383}$ Both of these studies point to tensile strain on $\mathrm{Cu}$ from lattice mismatch with the Pt substrate playing a role in the $\mathrm{CO}_{2} \mathrm{R}$ selectivity by altering the adsorption energy of reactive intermediates, as previously discussed. While these $C_{1} / C_{2}$ selectivity trends are interesting and merit further study to better understand, unfortunately it is clear that none of these bimetallics with highly HER active metals resulted in a more active $\mathrm{CO}_{2} \mathrm{R}$ catalyst for $>2 \mathrm{e}^{-}$products (Figure 36 in Section 7.4).

Interestingly, earlier reports from Watanabe et al. ${ }^{384,385}$ indicated that $\mathrm{Cu}-\mathrm{Ni}$ alloys could have synergistic methanol production because they found that neither of the pure metals produced methanol, but their $\mathrm{Cu}-\mathrm{Ni}$ alloys were shown to produce around 5-10\% Faradaic efficiency. The different structural properties of the bimetallic electrodes (incorporating $\mathrm{Ni}$ as an overlayer/surface additive $v s$. mixing as an alloy) might be the cause for the different products observed between this and the other reports discussed above. With improved product detection, however, methanol production has since been confirmed for both monometallic $\mathrm{Cu}$ and $\mathrm{Ni}$ electrodes by Kuhl et al. ${ }^{204} \mathrm{Thus,} \mathrm{it} \mathrm{is} \mathrm{unclear} \mathrm{what,}$ if any, synergistic effects were at play, and more thorough investigations are needed.

Palladium is somewhat unique in this grouping in that, according to Hori's data, Pd has very similar $\mathrm{H}_{2}$ and $\mathrm{CO}$ Faradaic efficiencies ( $\left.27 \%\right)$, although there is $\sim 40 \%$ of the current unaccounted for, which is likely from hydrogen absorption. ${ }^{1}$ The ease with which hydrogen can be absorbed into $\mathrm{Pd}$ metal has attracted interest in this material for $\mathrm{CO}_{2} \mathrm{R}$, and early studies showed that $\mathrm{Pd}$ selectivity was heavily dependent on whether it was employed as a hydrogen-depleted or hydrogen-enriched surface, 
with the latter leading to greatly enhanced formate production. ${ }^{386}$ Similarly, it was found that $\mathrm{Cu}-$ modified $\mathrm{Pd}$ and electrolytically charged $\mathrm{Pd}+\mathrm{H}$ electrodes displayed different selectivities, where $\mathrm{Cu} / \mathrm{Pd}+\mathrm{H}$ electrodes led to higher Faradaic efficiencies for the production of methane and particularly for formate compared to $\mathrm{Cu} / \mathrm{Pd}$ electrodes. ${ }^{387,388}$ This effect was enhanced when the amount of absorbed hydrogen in the $\mathrm{Pd}$ electrodes was larger $\left(\mathrm{Cu} / \mathrm{Pd}+\mathrm{H}\right.$ charged to $\left.20^{\circ} \mathrm{C} v s .100{ }^{\circ} \mathrm{C}\right)$, and when the coverage of $\mathrm{Cu}$ was lower, likely because higher $\mathrm{Cu}$ coverages prevented hydrogen from discharging through the cracks in the $\mathrm{Cu}$ film. ${ }^{387,388}$ Production of $\mathrm{CO}$ and $\mathrm{C}_{2+}$ products were not enhanced by the absorption of hydrogen. ${ }^{388}$ Another study found a complete shift in selectivity from $\mathrm{C}_{2} \mathrm{H}_{4}(32.1 \% \rightarrow 3.4 \%)$ to $\mathrm{C}_{2} \mathrm{H}_{6}$ $(<1 \% \rightarrow 30.1 \%)$ at $-1.0 \mathrm{~V}$ vs. $\mathrm{RHE}$ on a $\mathrm{Cu}_{2} \mathrm{O}$-derived catalyst after $\mathrm{Pd}(\mathrm{II})$-chloride was added to the electrolyte, while the production of other carbonaceous species remained almost unchanged. ${ }^{389}$ Mechanistic studies on this system lead the authors to suggest that $\mathrm{CO}_{2} \mathrm{R}$ on the $\mathrm{Cu}$ sites first produces $\mathrm{C}_{2} \mathrm{H}_{4}$, which is then hydrogenated to $\mathrm{C}_{2} \mathrm{H}_{6}$ with the assistance of adsorbed $\mathrm{PdCl}_{\mathrm{x}}$. Ultimately the practicality of this system may be limited since $\mathrm{PdCl}_{2}$ served as a sacrificial dopant, resulting in less than $100 \%$ total Faradaic efficiency due to reduction of the $\mathrm{PdCl}_{2}$ and decreasing $\mathrm{C}_{2} \mathrm{H}_{6}$ selectivity over time as this species was depleted. Regardless, both of these studies point to Pd serving as an active hydrogen source, shifting $\mathrm{CO}_{2} \mathrm{R}$ selectivity towards hydrogenated products.

While these bimetallics utilized $\mathrm{Pd}$ or $\mathrm{Cu}$ as substrates for deposition of the other component, $\mathrm{Cu}-\mathrm{Pd}$ alloys have also been reported and displayed a very different trend. Composition-dependent studies of Cu-Pd alloy nanoparticles ${ }^{390}$ and electrodeposited mesoporous films ${ }^{391}$ resulted in enhanced activity and selectivity to $\mathrm{CO}$, with optimum ratios of $\mathrm{Cu}: \mathrm{Pd}$ producing around $80 \% \mathrm{CO}$ at $-0.8 \mathrm{~V} v s$. RHE. Both studies attribute the enhanced $\mathrm{CO}$ production to a ligand effect, where $\mathrm{Cu}$ weakens the $\mathrm{CO}$ adsorption energy on adjacent Pd sites. Another reason for $\mathrm{CO}$ being the dominant $\mathrm{CO}_{2} \mathrm{R}$ product reported in these studies could be due to a lower $\mathrm{H}^{*}$ coverage resulting from $\mathrm{Pd}-\mathrm{H}$ bond weakening in Cu-Pd alloys, preventing CO hydrogenation. ${ }^{391,} 392$ While electronic effects may certainly have an impact, other studies suggest that geometric effects play the primary role in governing selectivity of $\mathrm{Cu}-\mathrm{Pd}$ bimetallics. A 1:1 ratio of CuPd gas diffusion electrodes was systematically studied in ordered, disordered, and phase separated atomic arrangements (Figure 32). ${ }^{393}$ Both intermetallic mixing patterns 
primarily made $\mathrm{CO}$ over a range of potentials, similar to the other two $\mathrm{Cu}-\mathrm{Pd}$ alloy systems reported, while the phase separated CuPd system showed a very clear difference in selectivity and made $>60 \%$ $\mathrm{C}_{2+}$ products. Furthermore, a compositional study of disordered $\mathrm{Cu}-\mathrm{Pd}$ electrodes also resulted in higher selectivity for $\mathrm{C}_{2+}$ products as the concentration of $\mathrm{Cu}$ increased from $\mathrm{Pd}<\mathrm{CuPd}_{3}<\mathrm{CuPd}<\mathrm{Cu}_{3} \mathrm{Pd}<\mathrm{Cu}$. ${ }^{393}$ Likely, the hydrogen absorption ability of Pd and the C-C coupling ability of $\mathrm{Cu}$ are both diminished upon increased mixing. Thus, bimetallic systems where $\mathrm{Cu}$ and $\mathrm{Pd}$ are sufficiently separated likely leads to more hydrogenated and/or $\mathrm{C}_{2+}$ products, whereas bimetallic systems with increased mixing between these two elements may lead to more $\mathrm{CO}$. While this provides compelling evidence for geometric effects driving the selectivity, increasing the degree of mixing should enhance any ligand effects as well. This is why, in general, it is extremely challenging to deconvolute ligand/strain effects from geometric effects.

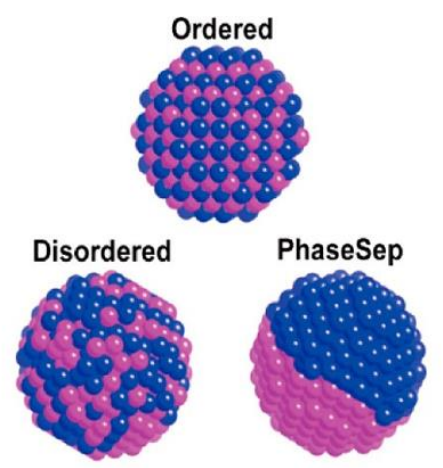

Figure 32: Schematics of ordered, disordered, and phase separated particles with 1:1 composition of CuPd. Reprinted from Ma et al. ${ }^{393}$ Copyright $@$ 2017, American Chemical Society.

\subsection{Copper and CO Producing Guest Metals (Ag, Au, Zn)}

Silver, gold, and zinc are all highly selective for $\mathrm{CO}$ production from $\mathrm{CO}_{2} \mathrm{R}$ in aqueous solutions under ambient conditions. ${ }^{1,85}$ As a result, many $\mathrm{Cu}-\mathrm{M}$ bimetallic electrodes (where $\mathrm{M}=\mathrm{Ag}, \mathrm{Au}, \mathrm{Zn}$ ) have shown increased selectivity, and in some cases higher intrinsic activity, for making CO. ${ }^{155,394-398}$ Alternatively, some $\mathrm{Cu}-\mathrm{M}$ bimetallic electrodes (where $\mathrm{M}=\mathrm{Ag}, \mathrm{Au}, \mathrm{Zn}$ ) have demonstrated synergistic effects leading to improved activity or selectivity for C-C coupled oxygenated products. ${ }^{73,123,128,130,132,399-403}$ The different factors leading to these two broader outcomes will be discussed subsequently. 


\subsubsection{Synergistic Effects for CO Production}

Several composition-dependent studies have shown increasing CO selectivity with increasing guest metal content, and this was demonstrated for a variety of bimetallic structures including: $\mathrm{Cu}-\mathrm{Ag}$ dendritic electrodeposits, ${ }^{394}$ small coverages of $\mathrm{Au}$ on $\mathrm{Cu},{ }^{395} \mathrm{Au}_{\mathrm{x}} \mathrm{Cu}_{1-\mathrm{x}}$ pulsed laser deposition alloys with and without $\mathrm{O}_{2}$ present, ${ }^{396} \mathrm{Au}_{\mathrm{x}} \mathrm{Cu}_{100-\mathrm{x}}$ bulk alloys, ${ }^{155}$ and $~ 10-11 \mathrm{~nm} \mathrm{Cu}-\mathrm{Au}$ alloy nanoparticles. ${ }^{397}$ This can be expected due to the weaker binding of $\mathrm{CO}$ on $\mathrm{Ag}$ and $\mathrm{Au}$, favoring $\mathrm{CO}$ desorption in these systems. ${ }^{277}$ There does exist some synergistic effect though, since several $\mathrm{Cu}$-Au bimetallics showed higher activities for CO production compared to pure Au electrodes tested in each study. ${ }^{155,397,398}$ Several explanations for this include: $\mathrm{CO}$ adsorbed on $\mathrm{Cu}$ sites can further promote $\mathrm{CO}$ desorption on $\mathrm{Au}$ sites due to dipole repulsion, ${ }^{155}$ or that neighboring $\mathrm{Cu}$ atoms can instead form a bond with the oxygen end of $\mathrm{COOH}$ via a bifunctional mechanism, stabilizing this intermediate for $\mathrm{CO}$ production on $\mathrm{Au}$ sites. ${ }^{397}$ It is unclear to what extent these may be true, but both suggest that an optimal surface $\mathrm{Cu}: \mathrm{Au}$ composition is required for enhanced CO activity in these alloys. It is worth noting that the optimal bulk composition was different in these two studies, but this could be due to a morphology effect for the planar $v s$. nanoparticle alloy configurations and/or the true surface compositions differed from the bulk.

Alternatively, a third study on $\mathrm{CuAu}(1: 1)$ alloy nanoparticles actually ascribed their enhanced $\mathrm{CO}$ activity to a compressively strained Au surface layer that formed as a result of a disorder-to-order transformation. ${ }^{398}$ The disordered $\mathrm{CuAu}$ nanoparticles were largely selective for $\mathrm{H}_{2}$, in agreement with the group's previous composition-dependent study, ${ }^{397}$ but the ordered $\mathrm{CuAu}$ nanoparticles were found to have higher specific and mass activities for CO production compared to pure Au nanoparticles. Since surface characterization revealed that a three-atoms-thick $\mathrm{Au}$ shell formed on top of the ordered $\mathrm{CuAu}$ alloy core, bifunctional effects were ruled out in this specific system; instead, it was suggested on the basis of a complementary DFT study that $6 \%$ compressive strain and vertical ligand effects contributed to the catalytic activity. ${ }^{398}$ While enhanced $\mathrm{CO}$ activity has been demonstrated in these $\mathrm{Cu}$ - $\mathrm{Au}$ alloy and core-shell systems, it is worth noting that none have shown a higher average turnover frequency than the state-of-the-art Au catalysts (Figure 37 in Section 7.4). Cu-Au bimetallics may offer an interesting 
opportunity to rationally design electrocatalysts yielding optimal and tunable syngas $\left(\mathrm{H}_{2}\right.$ and $\left.\mathrm{CO}\right)$ compositions, as demonstrated in a systematic study of $\mathrm{Cu}$ monolayer coverage on Au nanoneedles. ${ }^{133}$

Another report sought to take advantage of strain and vertical ligand effects by synthesizing $\mathrm{Au}$ core $\mathrm{Cu}$ shell nanoparticles $(\mathrm{Au} @ \mathrm{Cu})$ with different $\mathrm{Cu}$ shell thicknesses (7-10, 14-18, 30-40 layers). ${ }^{404}$ The cubic $\mathrm{Au}$ nanoparticle core resulted in epitaxial growth of $\mathrm{Cu}(100)$ for low and intermediate quantities of $\mathrm{Cu}$ layers, but this broke down for the largest number of $\mathrm{Cu}$ layers, which looked to be more (111) in character. Therefore, this system has several competing effects including strain, surface structure, roughness, and particle size differences that make it tough to ascribe the activity and selectivity to any one of these in particular. In addition, all three samples showed time-dependent selectivity over the course of an hour. ${ }^{404}$ While they do not confirm with structural characterization after electrochemistry, this could be due, at least in part, to $\mathrm{Cu}$ overlayer instability. Previous work investigating $\mathrm{Cu}$ overlayers on $\mathrm{Au}(100)^{405}$ and $\mathrm{Au}(111)^{405,406}$ observed alloy formation for $\mathrm{Cu}$ monolayers ${ }^{405,406}$ and multilayers ${ }^{406}$ under strongly cathodic potentials, while submonolayers ${ }^{405}$ relieved the interface tension by structural collapse. Thus, the significant (12.5\%) tensile strain between $\mathrm{Cu}$ and Au may simply be too unstable to yield a certain benefit, especially given the already high mobility of $\mathrm{Au}$ and $\mathrm{Cu}$ at room temperature. The Au overlayer on intermetallic $\mathrm{CuAu}$ nanoparticles generated by the disorder-to-order transformation resulted in an intermediate compressive strain (6\%) for $\mathrm{Au} .{ }^{398} \mathrm{The}$ reduced interfacial tension, plus the fact that the Au shell is already supported on an alloy phase, may decrease the driving force for overlayer instability. While extended tests over 12 hours do show some loss of activity (15\%) and gold content, ${ }^{398}$ near surface alloys could still be a promising paradigm to investigate moving forward for achieving stable strain effects.

\subsubsection{Synergistic Effects for $>2 e^{-}$Products}

There are two key effects in some $\mathrm{Cu}-\mathrm{M}$ bimetallic electrodes (where $\mathrm{M}=\mathrm{Ag}, \mathrm{Au}, \mathrm{Zn}$ ) that have led to improved activity or selectivity for C-C coupled and oxygenated products: $\mathrm{CO}$ spillover and electronic effects. In the first case, by co-locating domains of $\mathrm{Cu}$ with those of a $\mathrm{CO}$-generating metal, such as $\mathrm{Ag}, \mathrm{Au}$, and $\mathrm{Zn}$, additional $\mathrm{CO}$ can be supplied to $\mathrm{Cu}$ via eased surface diffusion over short 
length scales and/or by simply increasing the local CO concentration within the diffusion boundary layer, which in turn can lead to a higher coverage of $* \mathrm{CO}$. One potential demonstration of this concept was shown in a study on $\mathrm{Cu}-\mathrm{Ag}$ bimetallic electrodes using a pulsed $\mathrm{CO}_{2}$ electroreduction technique, which yielded higher Faradaic efficiencies for the production of $\mathrm{C}_{2+}$ compounds such as $\mathrm{CH}_{3} \mathrm{CHO}$, $\mathrm{C}_{2} \mathrm{H}_{5} \mathrm{OH}$, and $\mathrm{C}_{2} \mathrm{H}_{4}$ compared to pure $\mathrm{Cu}$ and $\mathrm{Ag}$ metal electrodes. ${ }^{123}$ By alternating cathodic and anodic biases, it was expected that desorption of $\mathrm{CO}$ occurred on $\mathrm{Ag}$ but not from $\mathrm{Cu}$, allowing for facile $\mathrm{C}-\mathrm{C}$ coupling on $\mathrm{Cu}$ due to a higher partial pressure of $\mathrm{CO}$ near the surface of the electrode. Another group electrodeposited $\mathrm{Cu}-\mathrm{Au}$ alloys onto a nanoporous $\mathrm{Cu}$ film (NCF) and found these led to higher alcohol selectivities compared to the NCF alone. ${ }^{399}$ They also compared the best $\mathrm{Cu}-\mathrm{Au}$ alloy composition electrodeposited on the NCF to that deposited on a flat copper plate and found that the alcohol selectivity was much lower using the copper plate substrate, suggesting that the nanostructured morphology and/or surface area has a strong impact on this effect. Linear sweep voltammetry in $\mathrm{CO}$ yielded a higher current than in Ar, so while CO spillover is not mentioned explicitly, the authors use this result to suggest that catalyzing conversion of intermediary $\mathrm{CO}$ could be the cause for the improved alcohol selectivity. ${ }^{399}$ Unfortunately, the results are only presented in terms of selectivity (Faradaic efficiency), no current density information for the bulk electrolysis was provided, so we were unable to determine if the intrinsic activity towards alcohols was improved.

There have been a few recent reports that have more directly linked observed improvements in $\mathrm{C}_{2+}$ activity and/or selectivity to a CO spillover effect. ${ }^{128,130,132,400,401}$ One system that clearly exhibited synergistic activity and selectivity for $\mathrm{C}_{2+}$ alcohols superior to that of $\mathrm{Cu}, \mathrm{Au}$, or $\mathrm{CuAu}$ alloys was a polycrystalline $\mathrm{Cu}$ foil decorated with Au nanoparticles $(\mathrm{Au} / \mathrm{Cu}) \cdot{ }^{400} \mathrm{In}$ fact, the $\mathrm{Au} / \mathrm{Cu}$ catalyst reduced the overpotential by $>260 \mathrm{mV}$ for the production of alcohols and had a $>100 \mathrm{x}$ higher rate of $\mathrm{CO}_{2}$ reduction to $>2 \mathrm{e}^{-}$products in this low overpotential region, as shown in Figure 33. These improvements are attributed to a tandem catalysis mechanism, where these two separate metals operate in close proximity with complementary surface chemistry. This hypothesis was supported by a control experiment performed on a surface alloy $\left(\mathrm{Au}_{0.01} \mathrm{Cu}_{0.99}\right)$, which did not reproduce the trend of enhanced alcohol production at low overpotentials. The $\mathrm{CO}$ spillover effect was further corroborated by transport modeling, which indicated that the $\mathrm{Au}$ nanoparticles provide a higher concentration of $\mathrm{CO}$ near the 
$\mathrm{Au} / \mathrm{Cu}$ surface than what $\mathrm{Cu}$ could produce alone or than what could be sustained through transport of $\mathrm{CO}$ from the bulk. ${ }^{400}$ Having this local $\mathrm{CO}$ source within the electrode double layer sidesteps the issue of low $\mathrm{CO}$ solubility in aqueous electrolytes, leading to the increased activity for $\mathrm{C}_{2+}$ products at low overpotentials. A similar $\mathrm{Au}$ on $\mathrm{Cu}$ system mentioned earlier ${ }^{395}$ only reported on electrolysis data at $1.85 \mathrm{~V}$ vs. SCE, in the high overpotential region where this enhancement in minimized. In addition, Morales-Guio et al. found that there was an optimal range for Au nanoparticle loading required to observe the synergistic effect to $>2 \mathrm{e}^{-}$products. ${ }^{400}$ These reasons, as well as differences in product detection capabilities, could possibly explain why this effect was not observed previously.
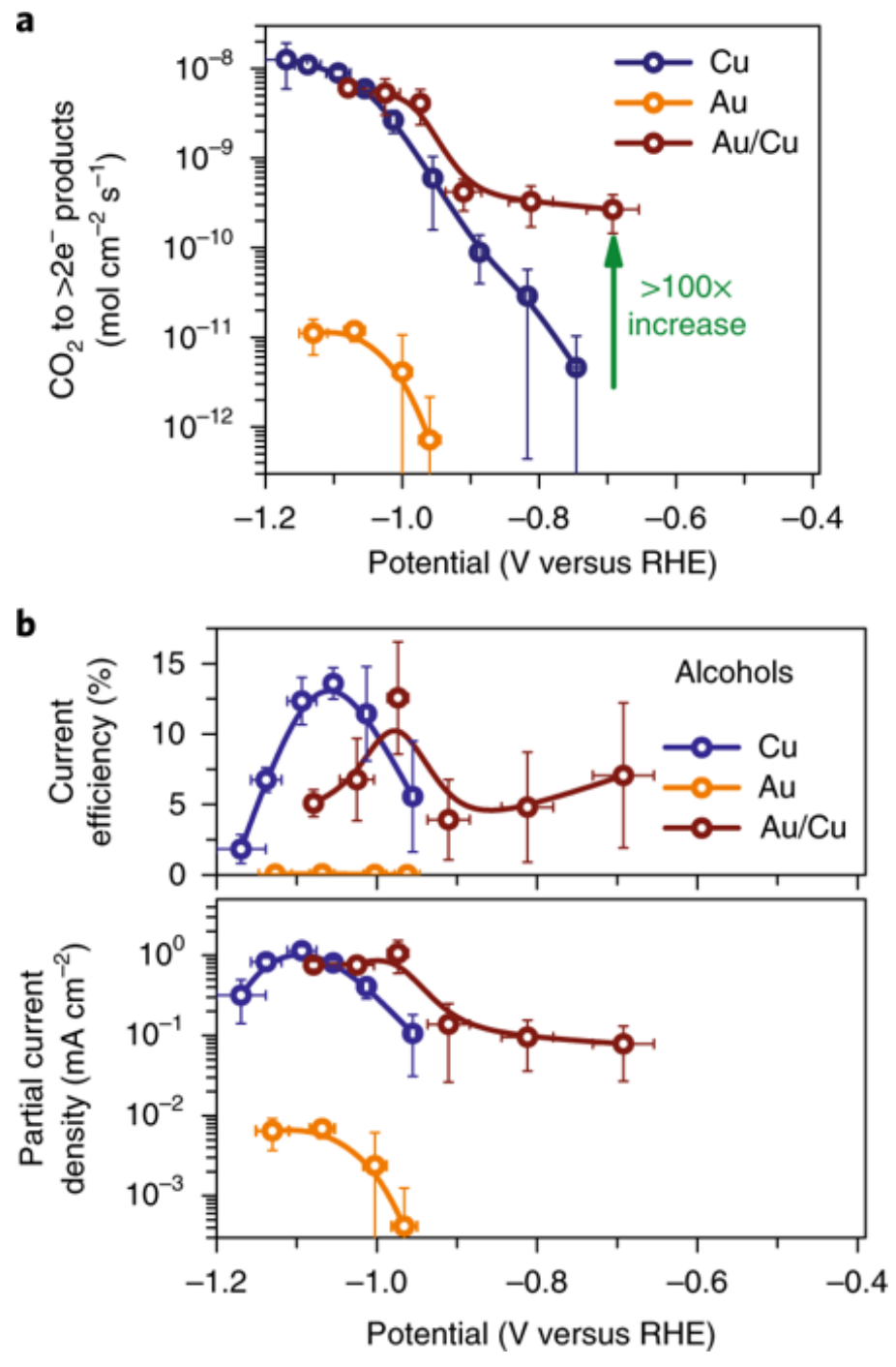

Figure 33: a) $\mathrm{CO}_{2} \mathrm{R}$ rate to $>2 \mathrm{e}^{-}$products; b) Current efficiencies and partial current densities to alcohols. Reprinted with permission from Morales-Guio et al. ${ }^{400}$ Copyright $\odot$ 2018, Springer Nature. 
Investigations of oxide-derived $\mathrm{Cu}_{\mathrm{x}} \mathrm{Zn}$ phase separated electrodes ${ }^{130}$ and $\mathrm{Ag}-\mathrm{Cu}_{2} \mathrm{O}$ phase blended and phase separated electrodes ${ }^{128}$ showed enhancements in $\mathrm{C}_{2+}$ selectivity, particularly for ethanol, and a CO spillover mechanism was again proposed. These bimetallic electrodes achieve some of the highest ethanol Faradaic efficiencies reported $\left(\mathrm{Ag}-\mathrm{Cu}_{2} \mathrm{O}\right.$ phase blended: $34 \%$ at $-1.2 \mathrm{~V} v s$. $\mathrm{RHE}^{128} ; \mathrm{Cu}_{4} \mathrm{Zn}: 29 \%$ at $-1.05 \mathrm{~V}$ vs. $\left.\mathrm{RHE}^{130}\right)$. It is noteworthy, however, that the enhancements in ethanol selectivity compared to their monometallic $\mathrm{Cu}$ control samples only occur at high overpotentials $(\mathrm{E} \leq-$ $0.9 \mathrm{~V} v s \mathrm{RHE})$. This is in stark contrast to the $\mathrm{Au} / \mathrm{Cu}$ system, which achieves higher selectivities at lower overpotentials (-1.0 V vs. RHE $\leq \mathrm{E} \leq-0.7 \mathrm{~V} v s$. RHE) and reaches a maximum in alcohol partial current density $90 \mathrm{mV}$ earlier than on $\mathrm{Cu}^{400}$ This can likely be rationalized by the higher overpotentials required for $\mathrm{CO}$ production on $\mathrm{Ag}$ and $\mathrm{Zn}$ compared to $\mathrm{Au}$ (see Table 3), ${ }^{1}$ which may shift the potential region where $\mathrm{CO}$ spillover can be a benefit. As for why ethanol is preferred over ethylene in these systems, it is likely that the lower rates of hydrogen production on the $\mathrm{Cu}_{\mathrm{x}} \mathrm{Zn}$ and $\mathrm{Ag}-\mathrm{Cu}_{2} \mathrm{O}$ electrodes and operation in the low overpotential region on $\mathrm{Au} / \mathrm{Cu}$ could shift the selectivity towards oxygenated C-C coupled products (i.e. ethanol) rather than hydrogenated C-C coupled products (i.e. ethylene). As discussed previously, while the selectivity is an important metric to consider, this alone does not tell you about the activity of the catalyst for $\mathrm{CO}_{2}$ reduction; for this, we must examine the (partial) current densities. Since the authors primarily discuss activity based on the geometric current density despite the different roughness factors (RF) of these catalysts ${ }^{130}$ or do not report the catalysts' $R F,{ }^{128}$ we will address their activity in Section 7.4.

Finally, it is worth discussing another report that has studied this tandem/sequential catalysis mechanism in detail using a series of microfabricated electrodes (Figure 34).$^{401}$ In the first design, separate, interdigitated $\mathrm{Au}$ and $\mathrm{Cu}$ electrodes on an insulating $\mathrm{SiO}_{2}$ substrate allow for each electrode to be actuated individually or simultaneously. When the electrodes were actuated simultaneously, there was a significant drop in the $\mathrm{CO}$ partial current density compared to when only Au was actuated, which suggests that the $\mathrm{CO}$ formed on $\mathrm{Au}$ lines is consumed by the $\mathrm{Cu}$ lines, validating the sequential catalysis concept. ${ }^{401}$ Interestingly, there was also a subtle shift in $\mathrm{C}_{2+}$ product distribution to favor oxygenates 
over ethylene production with decreasing $\mathrm{Cu}$ coverage, while all variations of the device maintained a lower oxygenates to ethylene ratio when only $\mathrm{Cu}$ was actuated.

(a) Interdigitated, side view

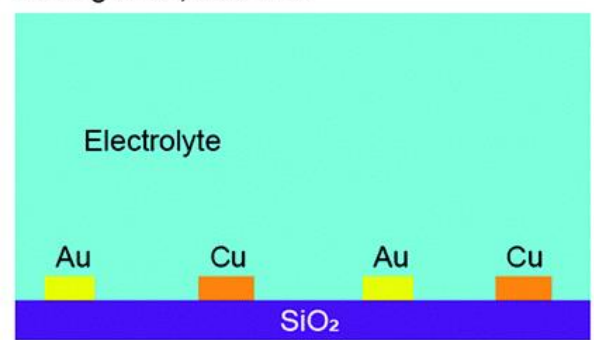

(c) dots, top view

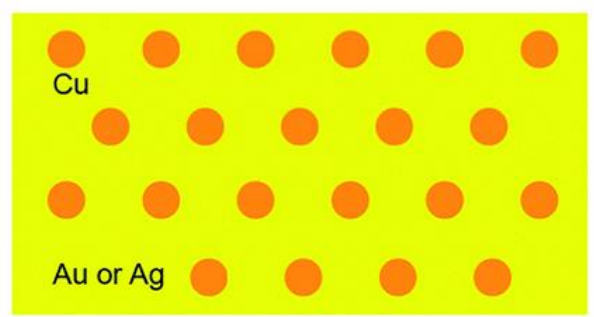

(b)

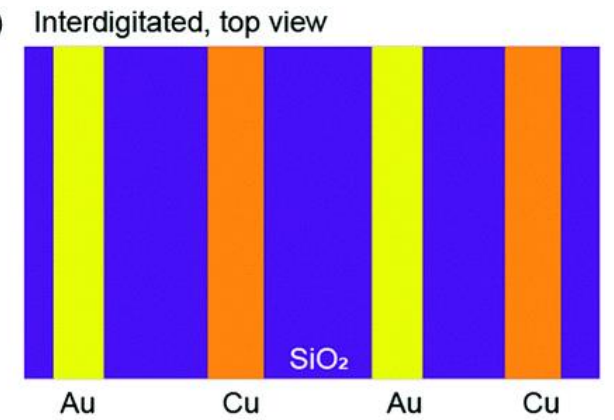

(d) lines, top view

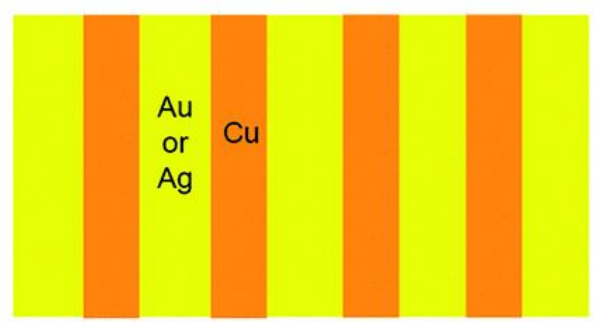

Figure 34: Schematics of interdigitated bimetallic electrodes, a) and b), and micropatterned electrodes, c) and d). Separation of the two metals in the interdigitated design allow for their independent operation. In all three designs, the areal ratio of the CO-producing metal, $\mathrm{Au}$ or $\mathrm{Ag}$, to $\mathrm{Cu}$ can be varied. Reproduced from Lum et al. ${ }^{401}$ with permission from the Royal Society of Chemistry.

The researches then probed this effect further by systematically fabricating a series of electrodes where $\mathrm{Cu}$ was patterned directly onto $\mathrm{Au}$ or $\mathrm{Ag}$ substrates, which allowed for fine control over the spacing and coverage of $\mathrm{Cu}$. As the coverage of $\mathrm{Cu}$ was decreased from $77.2 \%$ to $2.4 \%$, the ratio of oxygenates to ethylene Faradaic efficiency increased from 0.72 to 2.39 , which mainly resulted from a decrease in ethylene and increases in acetaldehyde, acetate, and propionaldehyde selectivities. ${ }^{401}$ Meanwhile, the total $\mathrm{C}_{2+}$ Faradaic efficiency was maintained in the 50-65\% range regardless of $\mathrm{Cu}$ coverage in these tandem/sequential catalyst devices; although, as one may expect, the partial current densities dropped with decreasing $\mathrm{Cu}$ coverage. Through a combination of control experiments and modeling, the reason behind the improved selectivity towards oxygenates in these devices was proposed to be due to $\mathrm{CO}$ transfer from the $\mathrm{Au}$ or $\mathrm{Ag}$ substrate inducing a higher coverage of $* \mathrm{CO}$ on the $\mathrm{Cu}$ surface. ${ }^{401}$ This increased $* \mathrm{CO}$ would likely come at the expense of ${ }^{*} \mathrm{H}$ coverage, which could result in slower surface hydrogenation rates and, in turn, favor the formation of oxygenates instead. All in all, the studies discussed thus far in this subsection showcase a new design principle for tandem or 
sequential catalysis; by employing a co-catalyst that generates an in-situ source of mobile $\mathrm{CO}, \mathrm{CO}_{2} \mathrm{R}$ to $>2 \mathrm{e}^{-}$products, particularly $\mathrm{C}_{2+}$ oxygenates, can be enhanced. Future work can advance tandem catalysis system designs by tuning the materials' surface structures, morphologies, and compositions.

Bimetallic $\mathrm{Cu}-\mathrm{Ag}$ thin films have also been reported to exhibit HER suppression and higher selectivity for $\mathrm{C}_{2+}$ oxygenates through other means. ${ }^{73,402}$ While $\mathrm{CO}$ spillover could still be a contributor in these systems, the dominant cause thought to be leading to these selectivity enhancements was tuning of adsorbate and reaction intermediate binding energies by incorporation of $\mathrm{Ag}$ into $\mathrm{Cu}$. In the first case, $\mathrm{CuAg}$ metal foils consisting of segregated $\mathrm{Cu}$ and $\mathrm{Ag}$ phases were prepared by arc melting, quenching, and cold rolling, and it was suggested that formation of $\mathrm{CuAg}$ surface alloys under electrochemical reaction conditions leads to compressive strain on $\mathrm{Cu}$ surface atoms. ${ }^{73}$ This compressive strain, as evidenced by an observable shift of the valence band structure of $\mathrm{Cu}$ to deeper levels, weakens the adsorption energy of $\mathrm{H}^{*}$, resulting in a $60-75 \%$ reduction in the HER activity. Interestingly, this was also correlated to a $10-15 \%$ increase in the total selectivity for $\mathrm{C}_{2+}$ products and a product distribution shift from hydrocarbons to oxygenates. Moreover, acetate and acetaldehyde accounted for a substantial portion of the $\mathrm{C}_{2+}$ oxygenate selectivity, reaching up to a combined $15 \%$ Faradaic efficiency, which is noteworthy given that these are typically minor $(<1 \%)$ products on pure $\mathrm{Cu}$. This selectivity modification is attributed to reduced rates of $\mathrm{C}-\mathrm{O}$ bond scission resulting from the HER suppression and the reduced oxophilicity of the compressively strained $\mathrm{Cu}$, which presumably inhibits the ability of $\mathrm{Cu}$ to reduce these carbonyl-containing products further. ${ }^{73}$ It is worth noting though that this did not impact the ability of $\mathrm{Cu}$ to make products derived from $\mathrm{CO}$ (i.e. the activity to $>2 \mathrm{e}^{-}$products remained unchanged when normalized to the nominal $\mathrm{Cu}$ composition).$^{73}$

In the second case, $\mathrm{CuAg}$ thin films across a range of nominal $\mathrm{CuAg}$ compositions were formed via physical vapor deposition, and many of the same trends were observed including HER suppression, decreased hydrocarbon selectivity, and increased oxygenate selectivity, particularly for the carbonyl products. ${ }^{402}$ In this case, however, the overall intrinsic $\mathrm{CO}_{2} \mathrm{R}$ activity toward further reduced products was decreased for $\mathrm{CuAg}$ versus $\mathrm{Cu}$. This is attributed to the significant nonequilibrium bulk-phase miscibility between $\mathrm{Ag}$ and $\mathrm{Cu}$ achieved in these films, ${ }^{402}$ compared to the previous work which had 
minimal miscibility of $\mathrm{Ag}$ in $\mathrm{Cu}\left(<3\right.$ at.\%). ${ }^{73}$ This significant $\mathrm{Ag}$ miscibility in $\mathrm{Cu}$ is also thought to be responsible for the observed selectivity enhancements by tuning the electronic structure of $\mathrm{Cu}$, decreasing the binding energy of $\mathrm{H}^{*}$ and oxygenated reaction intermediates. Further trends in product distribution were also discussed, including the significant suppression of $\mathrm{CH}_{4}$ leading to an overall higher selectivity for $\mathrm{C}-\mathrm{C}$ coupled products in these $\mathrm{CuAg}$ films compared to pure $\mathrm{Cu} .{ }^{402}$ With careful catalyst characterization, these studies demonstrate how formation of alloys using two metals that are thermodynamically immiscible under ambient conditions can be an effective strategy to alter $\mathrm{CO}_{2} \mathrm{R}$ activity (reduced HER) and selectivity (increased $\mathrm{C}_{2+}$ oxygenates) in bimetallic systems.

Another $\mathrm{Cu}-\mathrm{Ag}$ system has also demonstrated a higher selectivity for acetate formation ( $21 \%$ at $-1.33 \mathrm{~V} v s . \mathrm{RHE}$ at $\left.0{ }^{\circ} \mathrm{C}\right) .{ }^{403} \mathrm{In}$ this case, however, they were separate $\sim 6 \mathrm{~nm} \mathrm{Cu}$ and $\mathrm{Ag}$ nanoparticles immobilized on a polymer/glassy carbon electrode, and acetate was only observed over the bimetallic when benzotriazole (BTA) was in the electrolyte (over the $\mathrm{Cu}$ control sample it was observed in minor quantities, $<1 \%$ Faradaic efficiency, both with and without BTA). While the selectivity changed with the ratio of Ag present, indicating the Ag clusters may play an important role through the $\mathrm{CO}$ spillover mechanism discussed earlier, clearly the electrolyte additive has a dominant impact on the product formation since without BTA, the main $\mathrm{CO}_{2} \mathrm{R}$ product over the bimetallic electrode was $\mathrm{CO} .{ }^{403}$

\subsection{Copper and HCOO- Producing Guest Metals (In, Sn)}

The $p$-block metals are generally characterized by their high selectivity for formate production at high overpotentials. ${ }^{1,85}$ They also require a relatively high overpotential for the HER, which has drawn significant interest in employing them in bimetallic $\mathrm{Cu}$ systems as a strategy to reduce the selectivity for this competing side reaction. In addition, In and Sn electrodes have previously been demonstrated to exhibit a kinetically meta-stable oxide at the electrode/electrolyte interface at potentials where $\mathrm{CO}_{2} \mathrm{R}$ is observed, which can strongly influence the electrocatalytic properties of these systems. ${ }^{142,407}$ This also widens the range of surface chemistries that can be created by these bimetallic systems, so careful characterization of the electrode surface is crucial. Unfortunately, ex-situ characterization methods are 
often complicated by the oxophilicity of these elements and are particularly difficult given the nanostructured nature of the bimetallic catalysts in this section. Thus, comparisons and conclusions based on composition and/or oxidation state need to be taken with caution. In addition, it is impossible to identify which catalysts have a higher average turnover frequency because almost all of these studies reported current densities using the geometric electrode area. Thus, we will focus much of the remaining discussion of these $\mathrm{Cu}-\mathrm{M}$ bimetallics (where $\mathrm{M}=\mathrm{In}, \mathrm{Sn}$ ) on selectivity trends.

The key trends observed in $\mathrm{Cu}-\mathrm{M}$ (where $\mathrm{M}=\mathrm{In}, \mathrm{Sn}$ ) systems include synergistic $\mathrm{CO}$ production, HER suppression, and suppression of further reduced $\left(>2 \mathrm{e}^{-}\right)$products. ${ }^{408-419}$ In addition, several bimetallics maintained a higher selectivity to $\mathrm{HCOO}^{-408,409,420,421}$ In two of these cases, thick $\mathrm{Sn}$ deposits were employed on top of $\mathrm{Cu}$ substrates and resulted in overall Sn-like behavior with high selectivity (>80-90\%) to $\mathrm{HCOO}^{-}$formation. ${ }^{420,421}$ Thus, the $\mathrm{Cu}$ here mainly seems to serve as a support to provide a route for making high surface area Sn electrodes to increase the yield of $\mathrm{HCOO}^{-}$, rather than engendering any bimetallic effects. On the other hand, $\mathrm{Cu}$ core $\mathrm{SnO}_{2}$ shell nanoparticles $\left(\mathrm{Cu} @ \mathrm{SnO}_{2}\right)$ were investigated for $\mathrm{CO}_{2} \mathrm{R}$, and while the thicker $(1.8 \mathrm{~nm}) \mathrm{Sn}$ shell was again more selective for $\mathrm{HCOO}^{-}(<1 \% \mathrm{CO})$, the thinner shell $(0.8 \mathrm{~nm})$ instead resulted in very high selectivity for CO (>90\% from -0.6 to $-0.9 \mathrm{~V}$ vs. RHE) ${ }^{408}$ DFT calculations suggested that the combination of compression $(\sim 10 \%)$ due to the lattice mismatch between the core and shell and self-doping of $\mathrm{Cu}$ atoms diffusing out from the core resulted in the selectivity towards $\mathrm{CO}$ over $\mathrm{HCOO}^{-}$on $\mathrm{SnO}_{2}$. An analogous study on $\mathrm{Cu}$ core $\operatorname{In}_{2} \mathrm{O}_{3}$ shell nanoparticles reported similar results and reasoning; lattice compression and $\mathrm{Cu}$ doping of the $\operatorname{In}_{2} \mathrm{O}_{3}$ shell led to enhanced $\mathrm{CO}$ generation, particularly for the thinnest shell (68\% at $-0.7 \mathrm{~V} v s$. RHE), while the thickest shell had a higher selectivity for $\mathrm{HCOO}^{-}{ }^{418}$ These core-shell catalysts both show that the existence of $\mathrm{Cu}$ at the surface is an important factor governing the $\mathrm{CO}$ selectivity; there is further support for this design criteria in the studies discussed subsequently.

Two studies of $\mathrm{Sn}$ deposited on $\mathrm{Cu}$ oxide nanowires ${ }^{412}$ and oxide derived $\mathrm{Cu}$ substrates ${ }^{413}$ both demonstrated very high CO selectivity for optimized samples $\left(90 \% \mathrm{CO}\right.$ at $-0.8 \mathrm{~V} v$ s. $\mathrm{RHE}^{412}$; >90\% CO between -0.5 to $-0.8 \mathrm{~V}$ vs. $\mathrm{RHE}^{413}$ ), which drastically falls after there is enough $\mathrm{Sn}$ on the surface to surpass a full monolayer of coverage on $\mathrm{Cu}$. Similarly, a $\mathrm{Cu}$ foam sparsely decorated with $\mathrm{Sn}$ (2-3 at.\%) was found to achieve $>90 \% \mathrm{CO}$ selectivity from -0.6 to $-1.0 \mathrm{~V} v s$. RHE. ${ }^{419}$ Two studies of $\mathrm{Cu}-\mathrm{In}$ 
alloys synthesized from reduction of an oxide-derived $\mathrm{Cu}$ substrate in $\mathrm{In}_{\text {solution }}{ }^{414}$ and reduction of $\mathrm{CuInO}_{2}{ }^{415}$ also demonstrated high $\mathrm{CO}$ selectivity ( $>75 \% \mathrm{CO}$ between -0.4 to $-0.7 \mathrm{~V}$ vs. $\mathrm{RHE}^{414} ; 70 \%$ at $-0.8 \mathrm{~V}$ vs. $\mathrm{RHE}^{415}$ ). While the resulting bimetallic structures range from no evidence of alloy formation, ${ }^{412,419}$ to near surface alloy formation, ${ }^{413,414}$ to bulk alloy formation, ${ }^{415}$ similar reasoning for the enhanced CO selectivity is proposed; namely, it is thought that incorporation of $\mathrm{In} / \mathrm{Sn}$ disrupts favorable multi-Cu binding sites for protons while leaving $\mathrm{CO}$ binding sites unaffected, leading to HER suppression and enhanced CO selectivity at moderate overpotentials. ${ }^{412-415,419}$ This is supported by complementary DFT studies by the Takanabe group. ${ }^{413-415}$

Alternatively, it has also been proposed that a meta-stable $\operatorname{In}(\mathrm{OH})_{3}$ phase generated in-situ is responsible for enhanced $\mathrm{CO}$ selectivity in oxide-derived $\mathrm{Cu}$-In bimetallics. ${ }^{416} \mathrm{In}$ this report, several CuIn catalysts were synthesized from different starting oxide materials, and while they initially showed different performance \& morphologies, these catalysts evolved over repeated catalytic cycles to achieve similar CO selectivity $(\sim 50 \%)$ in parallel with appearance of an $\operatorname{In}(\mathrm{OH})_{3}$ shell. Furthermore, when $\mathrm{In}(\mathrm{OH})_{3}$ was etched off, the $\mathrm{CO}$ selectivity decreased until the hydroxide reappeared. ${ }^{416}$ Thus, it was hypothesized that the synergistic effect observed in these systems was due to the existence of bifunctional active sites at metal-oxide interfaces. Therefore, following this study, the same researchers conducted systematic experiments on a series of microfabricated electrodes seeking to establish a relationship between the $\mathrm{CO}$ activity and the length of these metal-oxide interfaces. ${ }^{410}$ By varying the amount of $\mathrm{In} / \mathrm{In}_{2} \mathrm{O}_{3}$ dots patterned onto $\mathrm{Cu} / \mathrm{Cu}_{2} \mathrm{O}$ substrates, the interfacial density $\left(\rho_{\text {int }}\right)$ could be varied across 2 orders of magnitude; meanwhile, the total geometric surface area of each single-phase component was kept constant so as to avoid contributions from differences in coverage. In this way, the researchers did observe a positive correlation between the $\mathrm{CO}$ activity and $\rho_{\text {int }}$ for $2 / 3$ of the variations studied $\left(\mathrm{In}_{2} \mathrm{O}_{3} / \mathrm{Cu}_{2} \mathrm{O}\right.$ and $\mathrm{In} / \mathrm{Cu}_{2} \mathrm{O}$, not $\left.\mathrm{In}_{2} \mathrm{O}_{3} / \mathrm{Cu}\right)$, but not for the reason they initially expected.

Ultimately, it was found that oxidic indium was not important, but the presence of $\mathrm{Cu}_{2} \mathrm{O}$, which was reduced to metallic $\mathrm{Cu}$ under reaction conditions, was necessary for this synergistic effect to occur. By contrast, all electrodes using a $\mathrm{Cu}$ substrate showed similar activity for $\mathrm{CO}$ regardless of $\rho_{\text {int }}$ (Figure 35a). ${ }^{410}$ As shown in Figure 35b, only the two electrode versions using $\mathrm{Cu}_{2} \mathrm{O}$ substrates displayed significant diffusion of In from the dots into the surroundings. Taken together, these results indicate 
that these In-modified $\mathrm{Cu}$ "halos" around the dots were responsible for the high activity for $\mathrm{CO}$ evolution rather than metal-oxide interfaces (both $\mathrm{Cu}$ and $\mathrm{In}$ were found to be metallic under reaction conditions anyway). ${ }^{410}$ Thus, with regards to their previous work, it is now suggested that the succession of reduction/oxidation cycles would aid in the formation of highly active, indium-poor metallic $\mathrm{Cu}$ compositions similar to those found in this study by accumulating $\operatorname{In}$ into an $\operatorname{In}(\mathrm{OH})_{3}$ sink; although the possibility of active sites existing at metal-oxide interfaces cannot be completely ruled out at this time. ${ }^{410}$
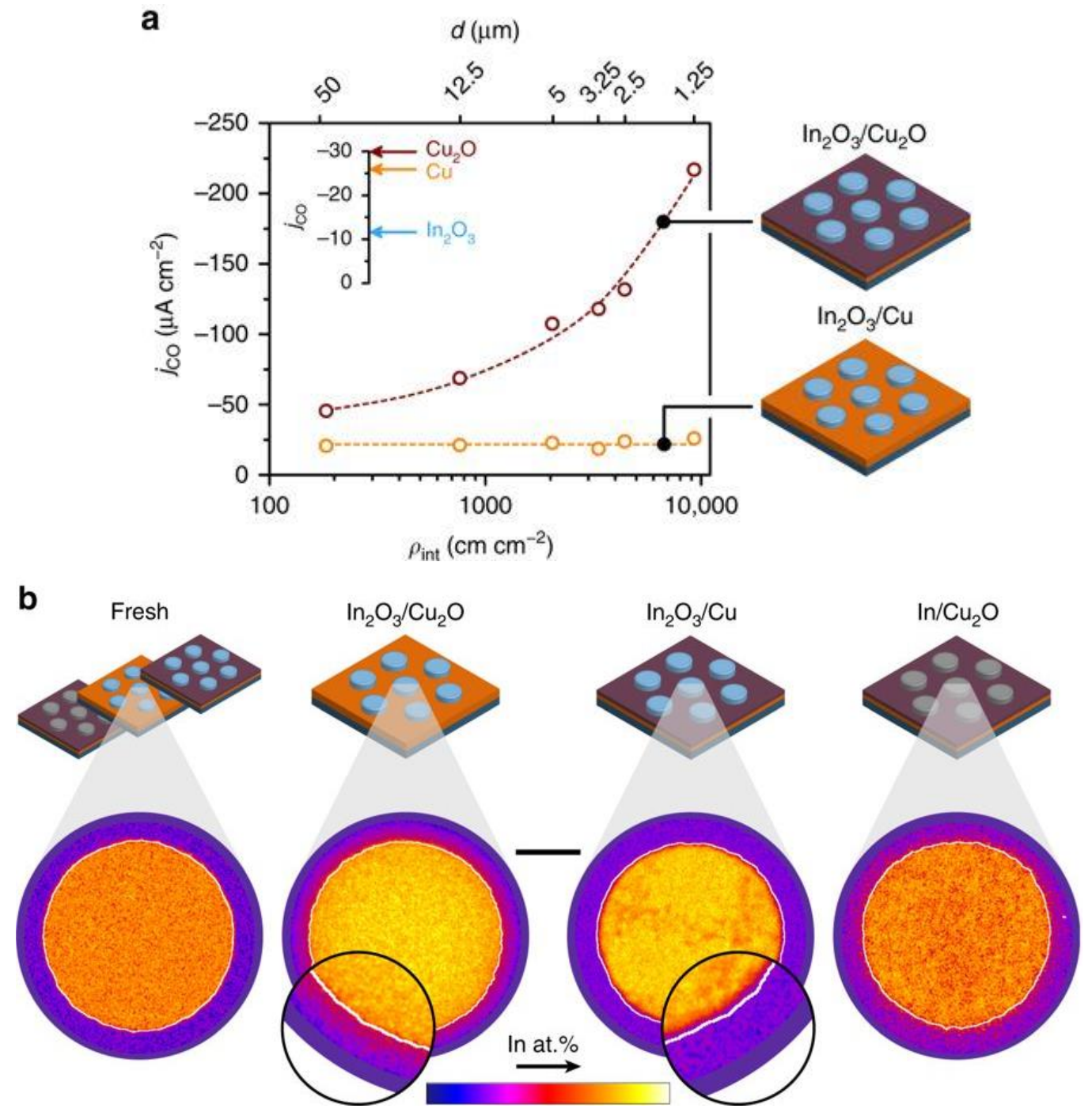

Figure 35: a) Partial current density for $\mathrm{CO}$ evolution ( $\mathrm{j}_{\mathrm{co}}$ ) over microfabricated $\operatorname{In}_{2} \mathrm{O}_{3} / \mathrm{Cu}_{2} \mathrm{O}$ and $\mathrm{In}_{2} \mathrm{O}_{3} / \mathrm{Cu}$ electrodes as a function of the interfacial density ( $\left.\rho_{\text {int }}\right)$ at $-0.6 \mathrm{~V} v s$. RHE. Note: the corresponding island diameter $(d)$ is indicated on the top x-axis, the trendlines were added as a visual aid, and the inset shows the corresponding partial current densities measured over the single-phase control samples. b) Elemental distribution of indium over individual islands for the 3 electrode variations $\left(\mathrm{In}_{2} \mathrm{O}_{3} / \mathrm{Cu}_{2} \mathrm{O}, \mathrm{In}_{2} \mathrm{O}_{3} / \mathrm{Cu}, \mathrm{In} / \mathrm{Cu}_{2} \mathrm{O}\right)$, with a representative island prior to the reaction shown as reference (Fresh). Note: the white contours indicating the border of the corresponding fresh islands were added as a visual aid, and the scale bar is $5 \mu \mathrm{m}$. Reprinted from Larrazábal et al. ${ }^{410}$ This work is licensed 
under a Creative Commons Attribution 4.0 International License (https://creativecommons.org/licenses/by/4.0/).

In light of this follow-up study by Larrazábal et al., it is clear that the interfaces between $\mathrm{In} / \mathrm{Sn}$ and $\mathrm{Cu}$ on the surface is emerging as a key factor governing the high $\mathrm{CO}$ selectivity observed in these bimetallic electrodes. This is further supported by a combined theory-experiment study on In-modified $\left(\mathrm{Cu}(\mathrm{OH})_{2}\right.$-derived $) \mathrm{Cu}$ nanowires. ${ }^{411}$ The composition where the highest number of $\mathrm{Cu}$-In interfacial sites were formed was found to be the optimal sample, achieving 93\% Faradaic efficiency for CO at 0.6 to $-0.8 \mathrm{~V}$ vs. RHE. DFT calculations also confirmed the crucial role of metallic $\mathrm{Cu}-\mathrm{In}$ interfaces in tuning the stability of key reaction intermediates; these interfacial sites were found to significantly decrease the barrier for $* \mathrm{COOH}$ formation while still allowing for facile release of $* \mathrm{CO}$, and weakly adsorbed $* \mathrm{H}$ and strongly adsorbed $* \mathrm{OCHO}$ on these sites could be the cause for suppression of $\mathrm{H}_{2}$ and $>2 \mathrm{e}^{-}$products. ${ }^{411}$

Finally, it is worth briefly revisiting the role of the oxide(s) in these bimetallic electrodes. Many of these studies suggested that the structure of the oxide precursor(s) governs the alloy formation and resulting CO activity several reasons including compositional ${ }^{417}$ and morphological effects. ${ }^{409,414,415}$ Interestingly, in the work by Larrazábal et al., a control experiment where indium was deposited on an already oxide-derived $\mathrm{Cu}$ substrate did not exhibit the same synergistic effect, which indicates that the in-situ reduction of the $\mathrm{Cu}_{2} \mathrm{O}$-based electrodes is required for diffusion of In to create the CO-selective, In-modified $\mathrm{Cu}$ "halos". ${ }^{410}$ This could perhaps explain the formation of highly active $\mathrm{Cu}-\mathrm{In}$ and $\mathrm{Cu}-\mathrm{Sn}$ surfaces that started with an oxide precursor mentioned earlier in this section. Although the oxidation state of $\mathrm{Cu}$, In, and $\mathrm{Sn}$ during $\mathrm{CO}_{2} \mathrm{R}$ is still an open area of debate, at least partial (if not full) reduction of these oxide species under reaction conditions seems beneficial to encourage diffusion of these components to create optimal interfacial sites. Unfortunately, untangling the complex tradeoff between the various possible effects in these nanostructured, bimetallic catalysts is incredibly difficult, so again, caution must be taken when trying to draw comparisons between systems or identify concrete structureactivity relationships. While there is clearly more work that needs to be done to better understand the factors governing the activity and selectivity of $\mathrm{Cu}-\mathrm{In}$ and $\mathrm{Cu}-\mathrm{Sn}$, these high surface area $\mathrm{Cu}$ bimetallics 
with p-block metals have certainly shown promising $\mathrm{CO}$ selectivities and geometric activities (Figure 37 in Section 7.4).

\subsection{Activity Comparisons}

In this chapter, we presented the main findings for different classes of $\mathrm{Cu}$-based bimetallic systems. Before reiterating the key selectivity trends and presenting activity comparisons, we wish to start by providing some overall perspectives on the challenges faced in studying and comparing the activity of bimetallic catalysts. First, as discussed in Section 2.4, benchmarking and reporting how the pure metal components perform in a given experimental setup is highly recommended to help validate trends observed for a given bimetallic catalyst. Second, identifying active sites on the surface of bimetallics under operando conditions can represent a major challenge. In particular, the composition at the surface of a bimetallic can be very different than in the bulk due to segregation of one component. This can be induced by fundamental differences in the materials' properties (oxophilicity, CO binding strength, miscibility, etc.) upon exposure to different environments (air ex-situ, electrolyte and/or applied potentials in-situ), as well as result from different catalyst synthesis methods and pretreatment procedures. Indeed, many studies discussed herein observed surface enrichment of one component either as a result of the synthesis $\operatorname{method}^{133,390,391,394,398,418}$ and/or electrochemical testing. ${ }^{73,382,391,396,405,406,414,416}$ Unfortunately, this makes comparisons between bimetallic catalysts more difficult; for example, two different bulk compositions may ultimately have similar surface compositions during electrolysis, and vice versa. This, in turn, can limit the scope of theoretical predictions and/or hinder our ability to match them with experimental results.

Third, while nanostructured bimetallics are interesting to explore in their own right, one must be careful when trying to draw structure-composition-activity relationships from these systems. Some reports on high surface area bimetallic electrodes noted different morphological features as a function of composition, which increases the difficulty of making systematic assessments. ${ }^{130,391,394,404,409}$ In addition, some studies tried testing a flat bimetallic catalyst to serve as a standard for comparison to their nanostructured catalyst, ${ }^{413,414}$ but this still has its pitfalls because the same surface ratio and 
dispersion of individual metal components may not be readily replicated between low and high surface area catalysts. In general, trying to decouple the impacts resulting from nanostructuring (increased surface area, faceting, grain boundaries, nanoparticle size and support effects, mass transport limitations) and those resulting from the bimetallic ensemble (composition/phase, atomic arrangement of each component, strain) is extremely difficult. Thus, when trying to draw conclusions about the intrinsic activity or selectivity of bimetallics, planar catalysts that minimize the effects of nanostructuring are ideal.

Finally, it is crucial to measure the ECSA of tested electrodes in order to determine whether a higher surface area catalyst truly has a greater average turnover frequency rather than greater activity simply resulting from an increased number of active sites. In light of the challenges in comparing bimetallic electrodes that were just discussed, getting an accurate measure of the ECSA of bimetallics can be especially difficult. As mentioned previously in Section 2.1, there exists a variety of methods for determining the ECSA, and researchers should keep in mind the limitations of each in order to choose the technique most suitable for the materials being studied. The most common method for estimating the ECSA of $\mathrm{CO}_{2} \mathrm{R}$ electrocatalysts has been to use double-layer capacitance $\left(\mathrm{C}_{\mathrm{dl}}\right)$ measurements. Ideally, a rough $\mathrm{Cu}-\mathrm{M}$ catalyst would be compared to a planar $\mathrm{Cu}-\mathrm{M}$ catalyst of the same surface composition; although as discussed earlier, this can be difficult to replicate and validate since changes in surface composition and morphology may also occur under reaction conditions. These discrepancies in composition may only have a small impact if the capacitance of the two materials are similar, but this introduces more uncertainty when the capacitance of the two materials are quite different. In the case of $\mathrm{Cu}-\mathrm{M}$ materials, since other $\mathrm{M}$ do not convert $\mathrm{CO}_{2}$ to $>2 \mathrm{e}^{-}$products to any appreciable degree, ECSA estimates for $\mathrm{Cu}-\mathrm{M}$ catalysts can compare the $\mathrm{C}_{\mathrm{dl}}$ of $\mathrm{Cu}-\mathrm{M}$ to the $\mathrm{C}_{\mathrm{dl}}$ of planar $\mathrm{Cu}$ when looking to see if the intrinsic activity has been improved beyond that of pure $\mathrm{Cu}$. In any case, it should be noted that the ECSA of bimetallic systems as determined by $\mathrm{C}_{\mathrm{dl}}$ ratios is typically a conservative estimate for the total number of active sites, since it is unlikely that the entire surface will be active in a bimetallic electrode. Still, any promotion in activity observed using this conservative estimate indicates that at least part of the enhancement is due to improved intrinsic reaction rates and not purely resulting from 
an increased number of active sites. Therefore, despite the difficulties and limitations, we urge researchers to make their best attempts to measure and report the ECSA of tested electrodes.

Unfortunately, only a limited number of $\mathrm{Cu}-\mathrm{M}$ bimetallics have been reported with the requisite information to compare catalytic activity on an average turnover frequency basis. Figures $\mathbf{3 6}$ and $\mathbf{3 7}$ provide the total $\mathrm{CO}_{2} \mathrm{R}$ current density to $>2 \mathrm{e}^{-}$products and $\mathrm{CO}$, respectively, normalized by both the exposed geometric area and ECSA when available for catalysts tested in aqueous-phase reactors. The few bimetallic $\mathrm{CO}_{2} \mathrm{R}$ catalysts that have been tested in vapor-fed reactors were not included for this direct comparison due to the vastly different reactant mass transport conditions. Also, in cases where multiple variations of $\mathrm{Cu}-\mathrm{M}$ bimetallics were studied, only the optimal material was generally included for clarity, in addition to the monometallic control sample if tested.

In Figure 36, both planar ${ }^{66}$ and high surface $\operatorname{area}^{90} \mathrm{Cu}$ control samples are included for reference. While a number of studies have reported bimetallics with increased selectivity to $>2 \mathrm{e}^{-}$ products, Figure 36a shows that this improvement is generally accompanied by a similar or lower geometric activity as compared to the corresponding $\mathrm{Cu}$ standard. It is worth noting that despite the decreased partial current densities, in some cases the $\mathrm{CO}_{2} \mathrm{R}$ energy efficiencies may be higher on the bimetallics compared to the $\mathrm{Cu}$ control samples. This was demonstrated by Lum et al.; the $4.3 \% \mathrm{Cu}$ dots on Ag sample shown in Figure 36a had the highest energy efficiency (21.4\%) for generation of $\mathrm{C}_{2+}$ products, while the $2.3 \% \mathrm{Cu}$ dots sample had the highest total $\mathrm{CO}_{2} \mathrm{R}$ energy efficiency $(32.8 \%){ }^{401}$

Looking at Figure 36b, it quickly becomes clear that the only aqueous-phase bimetallic catalyst system with a higher activity than pure $\mathrm{Cu}$ is the Au nanoparticles on $\mathrm{Cu}$ foil catalyst. ${ }^{400} \mathrm{The}$ OD-Cu $4 \mathrm{Zn}$ catalyst ${ }^{130}$ has a larger RF than the OD-Cu standard, so while it achieves an equivalent $\mathrm{CO}_{2} \mathrm{R}$ rate to $>2 \mathrm{e}^{-}$products when normalized by the geometric electrode area, this results in a lower average turnover frequency when normalized by the ECSA. Another bimetallic catalyst system that demonstrated higher activity than pure $\mathrm{Cu}$ was the $\mathrm{CuAg}$ wire reported by Hoang et al. ${ }^{132}$ While the $\mathrm{Cu}$ wire catalyst initially shows higher current densities to $\mathrm{C}_{2+}$ products at lower overpotentials then levels off (likely due to the lack of $* \mathrm{CO}$ resulting from the high turnover rate in this region), the $\mathrm{C}_{2+}$ activity of the $\mathrm{CuAg}$ wire catalyst continues to increase and surpasses that of the $\mathrm{Cu}$ wire at $-0.7 \mathrm{~V}$ vs. RHE. These results were not included in Figure 36 since they were obtained in a vapor-fed device, but it is worth noting the 
impressive $\mathrm{C}_{2+}$ activity and selectivity metrics achieved by the $\mathrm{CuAg}$ wire catalyst at this potential: $60 \%$ $\mathrm{C}_{2} \mathrm{H}_{4}$ and $25 \% \mathrm{C}_{2} \mathrm{H}_{5} \mathrm{OH}$ at a total current density of $\sim-300 \mathrm{~mA} / \mathrm{cm}^{2} .{ }^{132}$ This cannot be explained by surface area differences alone because the bimetallic has a 10\% higher ECSA but $60 \%$ higher current density than the $\mathrm{Cu}$ wire at this potential. Experiments where $\mathrm{CO}$ is mixed into the vapor feed during $\mathrm{CO}_{2} \mathrm{R}$ show that the $\mathrm{C}_{2+}$ saturation region displayed by the $\mathrm{Cu}$ wire catalyst disappears, and instead the current continues to increase like that of the $\mathrm{CuAg}$ wire. ${ }^{132}$ Therefore, $\mathrm{CO}$ spillover from Ag is likely a key mechanism by which the bimetallic wire achieves the improved performance at higher overpotentials. This once again highlights the potential of tandem catalyst systems that can effectively decouple multiple $\mathrm{CO}_{2} \mathrm{R}$ steps, bypassing design limitations intrinsic to monometallic electrodes.

In Figure 37, both planar ${ }^{66,422}$ and high surface area ${ }^{90,423} \mathrm{Cu}$ and $\mathrm{Au}$ control samples are included for reference. In Figure 37a we can see that many rough $\mathrm{Cu}$-based bimetallics have had activity comparable to pure $\mathrm{Au}$ on a geometric area-normalized basis. While this is encouraging for increasing cost-effectiveness for $\mathrm{CO}_{2} \mathrm{R}$ to $\mathrm{CO}$ catalysis by reducing noble metal usage, we can see in Figure 37b that pure $\mathrm{Au}$ is still orders of magnitude more active on an average turnover frequency basis. Also, it is interesting to note that the ECSA-normalized $\mathrm{CO}$ partial current densities for $\mathrm{Cu}-\mathrm{M}$ bimetallics with In or Sn essentially fall in between the planar and high surface area $\mathrm{Cu}$ standards; only those with Au have activity higher than $\mathrm{Cu}$, closer to that of Au. Thus, while many of these high surface area $\mathrm{Cu}-\mathrm{M}$ catalysts with p-block metals have demonstrated impressive CO selectivity (>90\%), the intrinsic activity for $\mathrm{CO}_{2} \mathrm{R}$ to $\mathrm{CO}$ on copper does not seem to have been enhanced in these systems. Again, the sample pool is very limited here though due to a lack of reporting the ECSA of these materials. Future studies on $\mathrm{Cu}-\mathrm{M}$ bimetallics should strive to include the roughness factors of these materials so that the intrinsic activity can be more readily compared. 


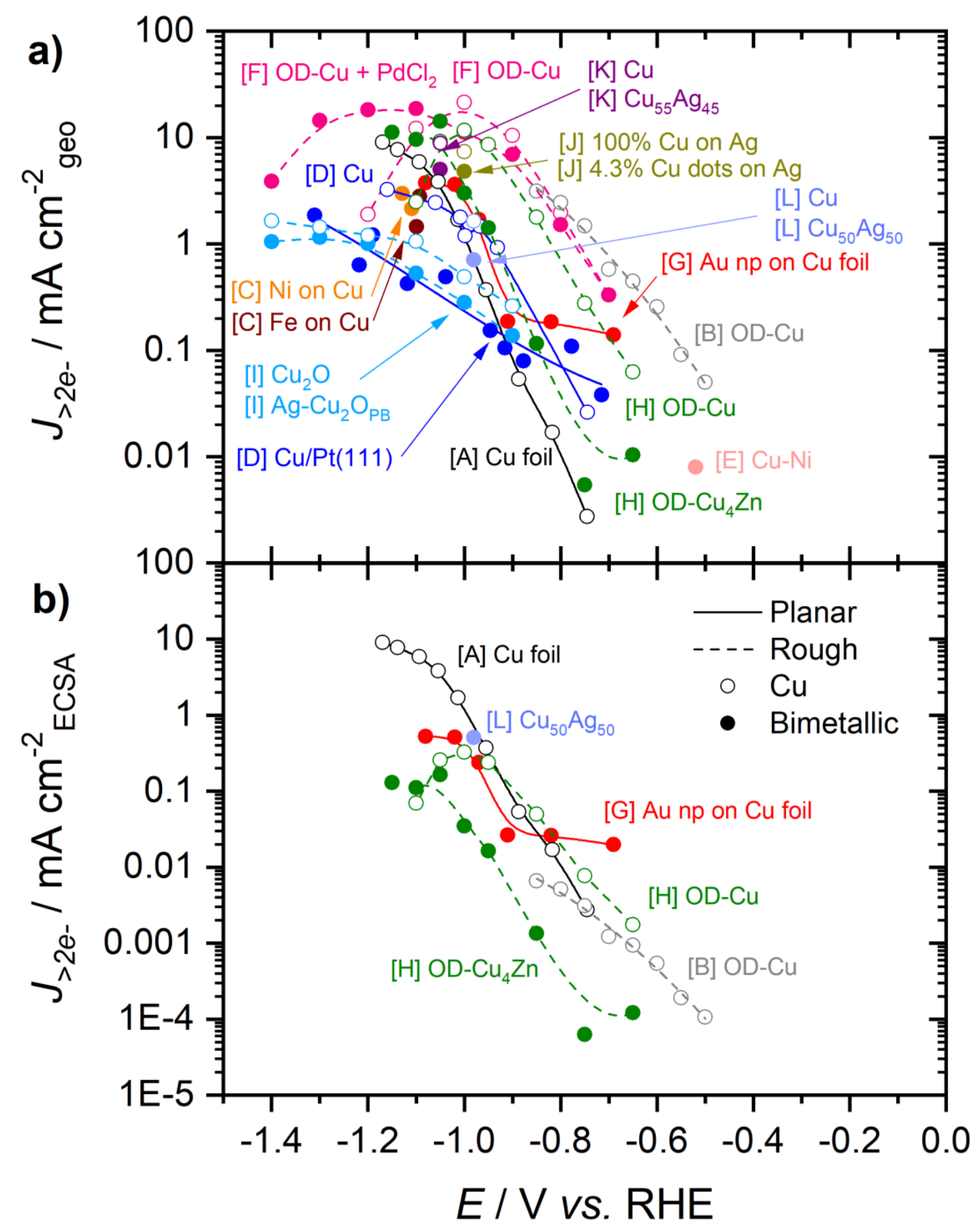

Figure 36: Cu-based bimetallic catalysts' total current density to further reduced $\left(>2 \mathrm{e}^{-}\right)$products normalized by a) the geometric area and $\mathbf{b}$ ) the ECSA. The data were obtained from the following studies: [A] Cu foil by Kuhl et al..$^{66}$; [B] OD-Cu by Li et al. ${ }^{90}$; [C] Ni and $\mathrm{Fe}$ deposits on $\mathrm{Cu}$ (two points shown for the beginning and end compositions formed by the in-situ electrodeposition) by Hori et al. ${ }^{378}$; [D] Cu overlayer on $\mathrm{Pt}(111)$ and $\mathrm{Cu}$ control sample by Varela et al. ${ }^{382}$; [E] $\mathrm{Cu}_{90.5} \mathrm{Ni}_{9.5}$ alloy by Watanabe et al. ${ }^{385}$; [F] OD-Cu with $\mathrm{PdCl}_{2}$ in the electrolyte forming deposits on the surface, as well as an OD-Cu control sample, by Chen et al. ${ }^{389}$; [G] Au nanoparticles on $\mathrm{Cu}$ foil by Morales-Guio et al. ${ }^{400}$; [H] OD$\mathrm{Cu}_{4} \mathrm{Zn}$ and OC-Cu control sample by Ren et al. ${ }^{130}$; [I] $\mathrm{Ag}_{-} \mathrm{Cu}_{2} \mathrm{O}_{\mathrm{PB}}(\mathrm{PB}=$ phase blended $)$ and $\mathrm{Cu}_{2} \mathrm{O}$ standard both deposited on Toray paper by Lee et al. ${ }^{128}$; [J] $4.3 \% \mathrm{Cu}$ dots patterned on an Ag substrate and a $100 \% \mathrm{Cu}$ (fully coated on Ag substrate) control sample by Lum et al. ${ }^{401}$; $[\mathrm{K}] \mathrm{Cu}_{55} \mathrm{Ag}_{45}$ bimetallic 
foil and $\mathrm{Cu}$ foil standard by Clark et al. ${ }^{73} ;[\mathrm{L}] \mathrm{Cu}_{50} \mathrm{Ag}_{50}$ thin film bimetallic and $\mathrm{Cu}$ control sample by Higgins et al. ${ }^{402}$

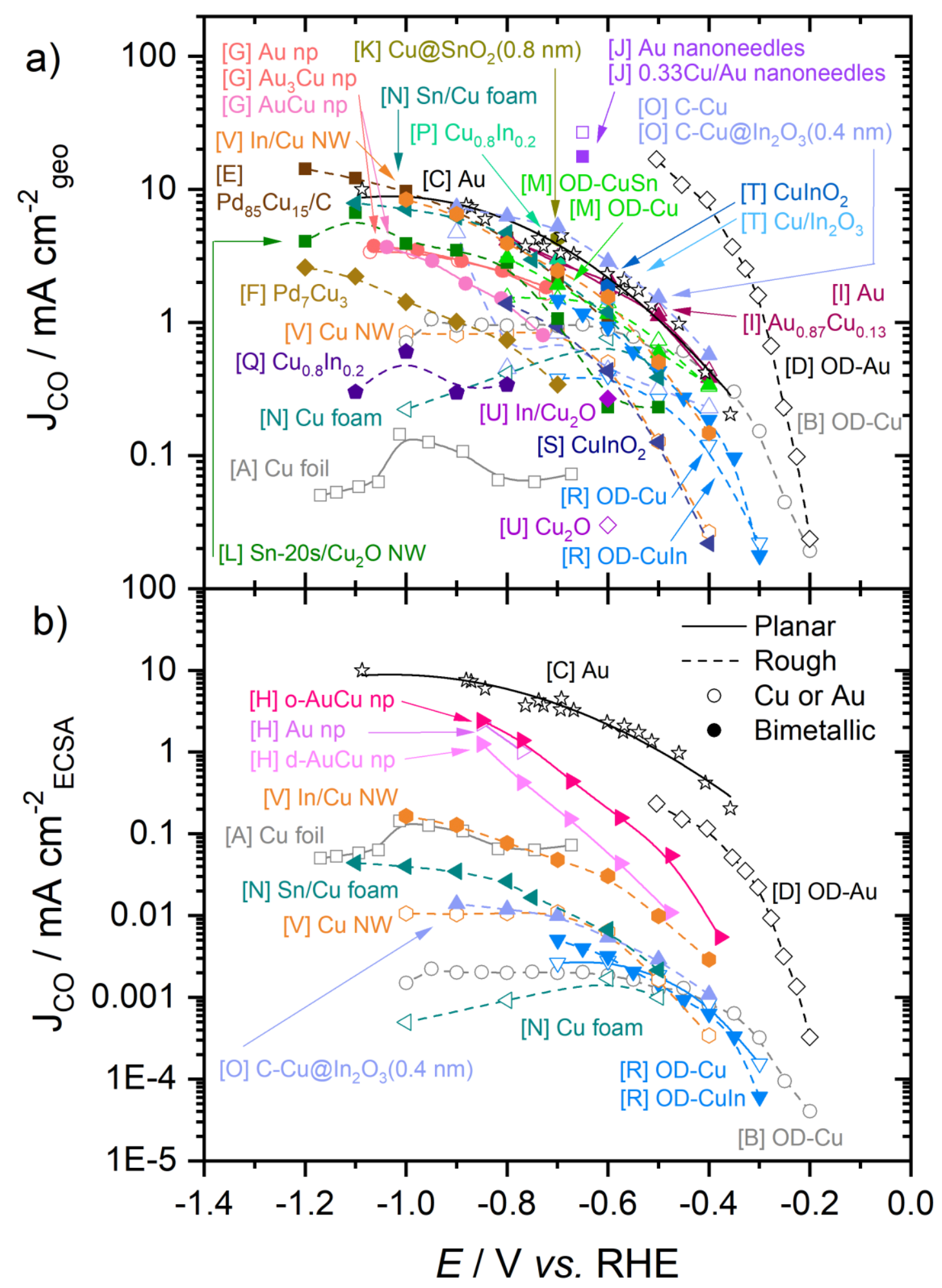


Figure 37: Cu-based bimetallic catalysts' total current density to $\mathrm{CO}$ production normalized by a) the geometric area and b) the ECSA. The data were obtained from the following studies: [A] $\mathrm{Cu}$ foil by Kuhl et al. ${ }^{66}$; [B] OD-Cu by Li et al. ${ }^{90}$; [C] Au by Hori et al. ${ }^{422}$; [D] OD-Au by Chen et al. ${ }^{423}$; [E] $\mathrm{Pd}_{85} \mathrm{Cu}_{15} / \mathrm{C}$ alloy nanoparticles supported on carbon black by Yin et al. ${ }^{390} ;[\mathrm{F}] \mathrm{Pd}_{7} \mathrm{Cu}_{3}$ electrodeposited mesoporous films by $\mathrm{Li}$ et al. ${ }^{391} ;[\mathrm{G}] \mathrm{AuCu}, \mathrm{Au}_{3} \mathrm{Cu}$, and Au nanoparticles by $\mathrm{Kim}$ et al. ${ }^{397} ;[\mathrm{H}]$ ordered (o-) and disordered (d-) AuCu nanoparticles and Au nanoparticle control sample by Kim et al. ${ }^{398}$; [I] $\mathrm{Au}$ and $\mathrm{Au}_{0.87} \mathrm{Cu}_{0.13}$ prepared by pulsed laser deposition in 220 mtorr $\mathrm{O}_{2}$ by Roy et al. ${ }^{396} ;[\mathrm{J}] 33 \% \mathrm{Cu}$ coverage on Au nanoneedles and Au nanoneedles by Ross et al. ${ }^{133} ;[\mathrm{K}] \mathrm{Cu} @ \mathrm{SnO}_{2}(0.8 \mathrm{~nm})$ core-shell nanoparticles by $\mathrm{Li}$ et al. ${ }^{408}$; [L] Sn deposited for $20 \mathrm{sec}$ on $\mathrm{Cu}_{2} \mathrm{O}$ nanowires by Zhao et al. ${ }^{412}$; [M] OD$\mathrm{CuSn}$ and OD-Cu control sample by Sarfraz et al. ${ }^{413}$; [N] 2-3 at.\% $\mathrm{Sn}$ on Cu10 foam (Sn/Cu foam) and $\mathrm{Cu} 10$ foam standard $\left(\mathrm{Cu} 10=10 \mathrm{~min}\right.$ electrodeposition) by Zeng et al. ${ }^{419}$; [O] Carbon-supported $\mathrm{Cu} @ \mathrm{In}_{2} \mathrm{O}_{3}(0.4 \mathrm{~nm})$ core-shell nanoparticles and carbon-supported $\mathrm{Cu}$ nanoparticle control sample by Xie et al. ${ }^{418}$; [P] $\mathrm{Cu}_{0.8} \mathrm{In}_{0.2}$ by He et al. ${ }^{417}$; [Q] $\mathrm{Cu}_{0.8} \mathrm{In}_{0.2}$ by Hoffman et al. ${ }^{409}$; [R] OD-CuIn and OD-Cu control sample by Rasul et al. ${ }^{414}$; [S] $\mathrm{CuInO}_{2}$-derived by Jedidi et al. ${ }^{415}$; [T] $\mathrm{Cu} / \mathrm{In}_{2} \mathrm{O}_{3}$-derived and $\mathrm{CuInO}_{2}$-derived by Larrazábal et al. ${ }^{416}$; [U] In dots microfabricated on $\mathrm{Cu}_{2} \mathrm{O}$ substrate $\left(\mathrm{In} / \mathrm{Cu}_{2} \mathrm{O}\right)$ and $\mathrm{Cu}_{2} \mathrm{O}$ control sample by Larrazábal et al. ${ }^{410}$; [V] In deposited on $\mathrm{Cu}$ nanowires $\left(\mathrm{Cu}(\mathrm{OH})_{2}\right.$ derived) using $20 \mathrm{mM} \mathrm{InCl}_{3}$ solution and $\mathrm{Cu}$ nanowire control sample by Luo et al. ${ }^{411}$

\subsection{Summary}

Here we examined $\mathrm{Cu}-\mathrm{M}$ bimetallic combinations across three groups, where the guest metal $\mathrm{M}$ is either highly selective to $\mathrm{H}_{2}, \mathrm{CO}$, or $\mathrm{HCOO}^{-}$. In all cases, the synergistic effects are generally diminished when the surface is dominated by the guest metal, causing the activity and selectivity to mimic that of the pure $\mathrm{M}$ component. For $\mathrm{H}_{2}$-selective metals, increased HER activity and decreased $\mathrm{CO}_{2} \mathrm{R}$ activity have typically been observed. Interestingly, the $\mathrm{C}_{2+}$ pathway(s) in particular get disrupted, as the main further reduced product formed in these systems was $\mathrm{CH}_{4}$, with $\mathrm{C}_{2} \mathrm{H}_{4}$ formation significantly suppressed. For CO-selective metals, high activity and selectivity for $\mathrm{CO}$ has been noted, as well as some synergistic activity and selectivity to $\mathrm{C}_{2+}$ products (especially oxygenates) resulting from $\mathrm{CO}$ spillover and electronic effects, respectively. Finally, for $\mathrm{HCOO}^{-}$-selective metals, HER suppression and high selectivity for $\mathrm{CO}$, along with inhibition of all further reduced products, have typically been observed.

At this time, it appears that the intrinsic activity of $\mathrm{Cu}$ (for $>2 \mathrm{e}^{-}$products) and $\mathrm{Au}$ (for $\mathrm{CO}$ ) have not been outmatched by bimetallic systems, except in the two demonstrations of tandem catalysis via $\mathrm{CO}$ spillover from $\mathrm{Au}$ nanoparticles on $\mathrm{Cu}$ foil ${ }^{400}$ and from $\mathrm{Ag}$ in a $\mathrm{CuAg}$ wire gas diffusion electrode. ${ }^{132}$ In these cases, the two monometallic catalysts operate in synergy whereby $\mathrm{Au} / \mathrm{Ag}$ provides a higher concentration of $\mathrm{CO}$ near the electrode surface than what $\mathrm{Cu}$ could produce alone or than what could 
be sustained through transport of $\mathrm{CO}$ from the bulk electrolyte, allowing for increased rates of $\mathrm{C}_{2+}$ product formation compared to pure $\mathrm{Cu}$. While there have been notable electronic and geometric effects demonstrated in $\mathrm{Cu}$ bimetallic systems, it appears that these have predominantly contributed to product distribution trends such as HER suppression, $>2 \mathrm{e}^{-}$products suppression, increased $\mathrm{CO}$ selectivity, and/or increased selectivity to $\mathrm{C}_{2+}$ oxygenates including ethanol or the carbonyl products. In these cases, weakening or disrupting sites for $* \mathrm{H}$ binding seems to be a key factor leading to enhanced $\mathrm{CO}_{2} \mathrm{R}$ selectivities. It is possible that poisoning of oxophillic sites by formate may prevent the predicted ability of bifunctional active sites to break scaling relations for $\mathrm{CO}_{2} \mathrm{R}$ to $>2 \mathrm{e}^{-}$products. ${ }^{203,366}$ Therefore, electrochemical $\mathrm{CO}$ reduction (COR) on $\mathrm{Cu}$-based bimetallics may be of interest for future work, since starting from $\mathrm{CO}$ as the reactant both eliminates the $\mathrm{HCOO}^{-}$pathway and allows for a broader range of $\mathrm{pH}$ conditions to be tested. Characterization and stability of these bimetallic system has also presented major challenges, as segregation and/or oxidation state changes of the individual metals can occur under reaction conditions and/or upon exposure to air for ex-situ characterization. Therefore, another area for future work includes further development of operando spectroscopic techniques to help elucidate the true chemical state and structure of active sites in $\mathrm{Cu}$ bimetallics during electrochemical $\mathrm{CO}_{2} \mathrm{R}$.

\section{Conclusions and Future Outlook}

Coupling electrochemical $\mathrm{CO}_{2}$ reduction $\left(\mathrm{CO}_{2} \mathrm{R}\right)$ with a renewable energy source to create high value fuels and chemicals is a promising strategy to help achieve a sustainable global energy economy. Not only could this serve as a renewable source of traditionally fossil-derived resources, but it could also enable storage of energy from intrinsically variable electricity generators such as wind and solar. In this review, we took a detailed look at the state of electrochemical $\mathrm{CO}_{2}$ reduction on $\mathrm{Cu}$ and the key levers that have been used to tune its activity \& selectivity, including surface faceting, electrolyte effects \& electrochemical conditions, nanostructuring, and preparation of bimetallics. First, we would like to emphasize the following key points:

1) Nanostructuring does not alter the intrinsic activity of copper in any significant manner, independent of the method used. While other morphological features may also play a role, 
we believe that increased surface area is the key factor altering the product selectivity of nanostructured materials, since this impacts the potential window where $\mathrm{CO}_{2} \mathrm{R} / \mathrm{COR}$ current densities are optimized.

2) a) Significant methods to increase the intrinsic activity of copper through bimetallic electronic or geometric effects have not yet been found. Notably, a tandem/sequential catalysis scheme has emerged as a promising paradigm for achieving higher activity and selectivity to $\mathrm{C}_{2+}$ products, particularly oxygenates. By co-locating domains of $\mathrm{Cu}$ with those of a $\mathrm{CO}$-generating metal (e.g. $\mathrm{Ag}, \mathrm{Au}$, and $\mathrm{Zn}$ ), additional $\mathrm{CO}$ can be supplied to the $\mathrm{Cu}$ surface.

b) Bimetallic electronic and geometric effects have, however, resulted in product distribution changes such as HER suppression, $>2 \mathrm{e}^{-}$products suppression, and/or increased $\mathrm{CO}$ selectivity compared to pure $\mathrm{Cu}$. It is also worth noting that higher selectivities to oxygenates such as acetate and acetaldehyde have been achieved in several $\mathrm{Cu}-\mathrm{Ag}$ systems.

3) It is important to consider the chemical potential of the reactants and electrolytes since this could easily change the catalyst surface structure and composition. The inherent mobility of copper and the ease with which it is oxidized present challenges in maintaining stable surfaces. This can explain the large observed dependency on the catalyst preparation and testing methods.

4) Theoretical studies have indicated that step sites may very well dominate $\mathrm{CO}_{2} \mathrm{R}$ activity, although experimental validation of this remains a challenge. Copper surfaces have been shown to reconstruct under reaction conditions. Thus, the formation of undercoordinated defects or step sites during preparation or testing of single crystal electrodes could explain why they have demonstrated similar overall activity.

5) It is crucial to report the electrochemically active surface area (ECSA) of tested electrodes whenever possible in order to determine if greater catalytic activity can be attributed to improved intrinsic activity (higher average turnover frequency) rather than an increased number of active sites (larger surface area). It is also important to keep in mind the ambiguities of measuring ECSA and choose the method most appropriate for the system at hand. 
6) Catalysts with different roughness factors and/or catalysts tested under different electrolyte and electrochemical conditions will reach mass transport limitations at different rates. Thus, it is not always straightforward to compare electrodes tested at the same applied potential, since these different conditions may lead to different kinetic overpotentials. When investigating the intrinsic behavior of novel electrocatalyst materials, we underline the importance of comparing data in potential windows governed by the intrinsic reaction kinetics, so as not to convolute the impacts of catalyst properties and mass transport effects.

Electrochemical $\mathrm{CO}_{2}$ reduction on copper catalysts holds significant promise, but there is still much work to be done before low-cost, scalable deployment of $\mathrm{CO}_{2} \mathrm{R}$ technologies can be realized. Towards this end, we not only need greater fundamental understanding of $\mathrm{CO}_{2} \mathrm{R}$ mechanisms, but increased practical performance metrics as well. We will briefly discuss our outlook on key areas for future studies in these spaces.

To date, most of the catalyst characterization techniques have been conducted ex-situ before and/or after $\mathrm{CO}_{2} \mathrm{R}$ due to the complexity of the electrified electrode/electrolyte interface. There has been an increasing trend, however, in the development and implementation of in-situ/operando characterization techniques, with recent studies providing new insights highlighted in each section of this review. We urge the further advancement of these techniques, as they can provide crucial insights regarding the catalyst surface structure, composition, oxidation state, and adsorbed intermediates under reaction conditions. These will be invaluable tools for providing better understanding of active sites, elucidation of reaction mechanisms, and validation of predictions from theoretical studies. For more details on the state of surface characterization techniques for $\mathrm{CO}_{2} \mathrm{R}$ and the progress in this area of research, we direct the reader to a reference module by Soriaga et al. ${ }^{424}$ in addition to a recent review article by Handoko et al. ${ }^{10}$

The developments in theoretical modeling of the electrochemical interface have yielded significant progress in identifying key active site motifs, reaction intermediates and descriptors, solvation and ion promotion effects, and $\mathrm{CO}_{2} \mathrm{R}$ pathways. While much of the previous focus has been 
on analyzing the reaction energetics and thermodynamics, further developments in kinetic modeling will be required to better understand the interplay between coverage, potential dependence of activation energies, adsorbate-adsorbate interactions, and $\mathrm{pH}$ effects. The development of machine-learning algorithms also presents an opportunity to develop predictive capabilities and screening methods, which can aid materials discovery efforts and promote the rational design of $\mathrm{CO}_{2} \mathrm{R}$ catalysts. ${ }^{425-427}$ Challenges remain in the reconciliation of conclusions between existing theoretical approaches, the advancement of functionals for describing charge at the electrochemical interface, and the evaluation of kinetics and reaction barriers of elementary steps under reaction conditions. Multi-scale modeling of transport effects will also be critical for better understanding of interfacial processes, especially for more practical high surface area catalysts and vapor-fed reactors. These developments will lead to increased and improved coupling between experimental and theoretical $\mathrm{CO}_{2} \mathrm{R}$ studies.

Next, we feel that the field must begin developing the necessary catalysts and reactor systems to enable scalable (low cost), selective and energy efficient conversion of $\mathrm{CO}_{2}$ to useful fuels and chemicals. It is often cited how $\mathrm{Cu}$ typically requires $\sim 1 \mathrm{~V}$ overpotential to form valuable hydrocarbons and alcohols, ${ }^{1}$ but this mainly applies to planar systems. Nanostructuring, along with the use of alkaline electrolytes and larger-sized alkali cations, has provided a route to decreasing the required overpotential and altering the selectivity to certain products. Most notably, immense progress has been made in recent years in development of gas diffusion electrode (GDE) and continuous flow devices. ${ }^{13,127,132,293,331,348,351-}$ ${ }^{355,428}$ For example, a recent report achieved a very high $\mathrm{C}_{2} \mathrm{H}_{4}$ selectivity of $\sim 70 \%$ over 150 hours of operation between 75 and $100 \mathrm{~mA} / \mathrm{cm}^{2}$, and a full-cell energy efficiency for $\mathrm{CO}_{2}$ conversion to $\mathrm{C}_{2} \mathrm{H}_{4}$ of $34 \% .{ }^{352}$ While this value is lower than the $60-80 \%$ energy efficiency typically achieved for water splitting, this is quite reasonable based on the relative maturity of that field, as well as the significant difference in potential product values. Thus, while there is always a need for fundamental research, the field is at a stage where we can start accelerating the development of practical devices. This includes optimization of device components (catalysts, supports, membrane, electrolyte) and conditions (flow rate, temperature, pressure, etc.), as well as testing the tolerance to more realistic conditions. For example, many potential $\mathrm{CO}_{2}$ sources for initial commercial applications (such as industrial plant flue 
gas, biogas, etc.) will have diluted $\mathrm{CO}_{2}$ streams with different potential contaminants. ${ }^{429}$ Renewablypowered $\mathrm{CO}_{2} \mathrm{R}$ may also have interrupted/variable power input depending on the source (such as intermittent wind \& solar), so tolerance to factors like these must be taken into account for large scale deployment of $\mathrm{CO}_{2} \mathrm{R}$ technology. In addition, complicated product separations may result in significant additional energy requirements, which should ideally be minimized. ${ }^{429}$

Lastly, we will briefly discuss three other focus areas for improving $\mathrm{CO}_{2} \mathrm{R}$ activity \& selectivity. The competing HER remains a significant issue for $\mathrm{CO}_{2} \mathrm{R}$ in aqueous systems. While progress has been made in improving $\mathrm{CO}_{2} \mathrm{R}$ selectivity by employing basic electrolytes, we additionally suggest the investigation of $\mathrm{CO}_{2} \mathrm{R}$ in nonaqueous and solid polymer electrolytes. While this review focused on $\mathrm{Cu}$ catalysts, there are also several emerging classes of catalysts that hold the potential to break scaling relations between reaction intermediates, including stable alloys, single atom catalysts, (doped) carbon materials, metal organic frameworks (MOFs) and covalent organic frameworks (COFs), materials with 3 dimensional active sites that can induce confinement effects, and functionalized surfaces (tethering promoters/ligands to the electrode surface).$^{58,203,374,430}$ Materials discovery efforts should continue in these directions to identify novel catalyst materials with enhanced properties. Finally, CO reduction has been increasingly studied in recent years, both as a proxy to better understand $\mathrm{CO}_{2}$ reduction mechanisms, as well as for fundamental understanding of this important reaction in its own right. If viable COR catalysts are developed, this could enable tandem or cascaded reaction systems in which $\mathrm{CO}_{2} \mathrm{R}$ is split into two separate steps: 1) $\mathrm{CO}_{2}$ reduction to $\mathrm{CO}$, then 2) $\mathrm{CO}$ reduction to the desired product(s). Unique synergies could also be achieved by coupling $\mathrm{CO}_{2}$ electroreduction with alternate types of catalytic processes (biological, thermal, etc.).

In summary, while many challenges and opportunities remain in developing catalysts and reactor systems with high activity, selectivity, stability, and scalability, much progress has also been attained in recent years, priming this technology for commercial application. With continued research $\&$ development, electrochemical $\mathrm{CO}_{2}$ reduction could have a substantial impact on the sustainability of our global energy economy. 


\section{Acknowledgements}

The material from Stanford/SLAC researchers is based upon work performed by the Joint Center for Artificial Photosynthesis, a DOE Energy Innovation Hub, supported through the Office of Science of the U.S. Department of Energy under Award No. DE-SC0004993.

The work by researchers at the Technical University of Denmark was supported by a research grant (9455) from VILLUM FONDEN.

B.S. also acknowledges a grant from Innovation Fund Denmark (8057-00018B ECOEthylene).

\section{Supporting Information}

1. Thermochemistry: Raw Data and Calculations. Includes Table S1: Thermochemistry data for carbon products and copper phases, calculation of equilibrium potentials, energy content of carbon products calculations, and discussion/calculations of $\mathrm{pH}$ dependence on the RHE and SHE scales.

2. Economics: Raw Data and Calculations. Includes descriptions of global non-energy carbon in industry, economics of $\mathrm{CO}_{2}$ reduction products

3. Computational Studies: Energetics and Kinetics of Elementary Steps. Includes Table S2: Calculated barriers for $\mathrm{CO}_{2} \mathrm{R}$ to $\mathrm{CO}$ via various methods, Table S3: Calculated barriers for $\mathrm{CO}$ reduction to $\mathrm{C}_{1}$ products via various methods, Table S4: Calculated barriers for $\mathrm{C}-\mathrm{C}$ coupling via various methods.

\section{References}

(1) Hori, Y. Electrochemical CO2 Reduction on Metal Electrodes. In Modern Aspects of Electrochemistry; Vayenas, C. G., White, R. E., Gamboa-Aldeco, M. E., Eds.; Springer, 2008; Vol. 42, pp 89-189.

(2) Gattrell, M.; Gupta, N.; Co, A. A Review of the Aqueous Electrochemical Reduction of CO2 to Hydrocarbons at Copper. J. Electroanal. Chem. 2006, 594, 1-19.

(3) Vasileff, A.; Xu, C.; Jiao, Y.; Zheng, Y.; Qiao, S.-Z. Surface and Interface Engineering in Copper-Based Bimetallic Materials for Selective CO2 Electroreduction. Chem 2018, 4, 18091831.

(4) Gamler, J. T. L.; Ashberry, H. M.; Skrabalak, S. E.; Koczkur, K. M. Random Alloyed versus Intermetallic Nanoparticles: A Comparison of Electrocatalytic Performance. Adv. Mater. 2018, $30,1801563$.

(5) Birhanu, M. K.; Tsai, M.-C.; Kahsay, A. W.; Chen, C.-T.; Zeleke, T. S.; Ibrahim, K. B.; Huang, C.-J.; Su, W.-N.; Hwang, B.-J. Copper and Copper-Based Bimetallic Catalysts for Carbon Dioxide Electroreduction. Adv. Mater. Interfaces 2018, 5, 1800919.

(6) Xie, H.; Wang, T.; Liang, J.; Li, Q.; Sun, S. Cu-Based Nanocatalysts for Electrochemical Reduction of CO2. Nano Today 2018, 21, 41-54. 
(7) Gu, Z.; Shen, H.; Shang, L.; Lv, X.; Qian, L.; Zheng, G. Nanostructured Copper-Based Electrocatalysts for CO2 Reduction. Small Methods 2018, 2, 1800121.

(8) Rendón-Calle, A.; Builes, S.; Calle-Vallejo, F. A Brief Review of the Computational Modeling of $\mathrm{CO} 2$ Electroreduction on Cu Electrodes. Curr. Opin. Electrochem. 2018, 9, 158-165.

(9) Raciti, D.; Wang, C. Recent Advances in CO2 Reduction Electrocatalysis on Copper. ACS Energy Lett. 2018, 3, 1545-1556.

(10) Handoko, A. D.; Wei, F.; Jenndy; Yeo, B. S.; Seh, Z. W. Understanding Heterogeneous Electrocatalytic Carbon Dioxide Reduction through Operando Techniques. Nat. Catal. 2018, $1,922-934$.

(11) Lee, C. W.; Yang, K. D.; Nam, D.-H.; Jang, J. H.; Cho, N. H.; Im, S. W.; Nam, K. T. Defining a Materials Database for the Design of Copper Binary Alloy Catalysts for Electrochemical CO2 Conversion. Adv. Mater. 2018, 30, 1704717.

(12) Arán-Ais, R. M.; Gao, D.; Roldan Cuenya, B. Structure- and Electrolyte-Sensitivity in CO2 Electroreduction. Acc. Chem. Res. 2018, 51, 2906-2917.

(13) Jhong, H. R. M.; Ma, S.; Kenis, P. J. A. Electrochemical Conversion of CO2 to Useful Chemicals: Current Status, Remaining Challenges, and Future Opportunities. Curr. Opin. Chem. Eng. 2013, 2, 191-199.

(14) Xu, S.; Carter, E. A. Theoretical Insights into Heterogeneous (Photo)Electrochemical CO 2 Reduction. Chem. Rev. 2018, 10.1021/acs.chemrev.8b00481.

(15) Qiao, J.; Liu, Y.; Hong, F.; Zhang, J. A Review of Catalysts for the Electroreduction of Carbon Dioxide to Produce Low-Carbon Fuels. Chem. Soc. Rev. 2014, 43, 631-675.

(16) Kortlever, R.; Shen, J.; Schouten, K. J. P.; Calle-Vallejo, F.; Koper, M. T. M. Catalysts and Reaction Pathways for the Electrochemical Reduction of Carbon Dioxide. J. Phys. Chem. Lett. 2015, 6, 4073-4082.

(17) Zhu, D. D.; Liu, J. L.; Qiao, S. Z. Recent Advances in Inorganic Heterogeneous Electrocatalysts for Reduction of Carbon Dioxide. Adv. Mater. 2016, 28, 3423-3452.

(18) Zhang, B.; Zhang, J. Rational Design of Cu-Based Electrocatalysts for Electrochemical Reduction of Carbon Dioxide. J. Energy Chem. 2017, 26, 1050-1066.

(19) Vickers, J. W.; Alfonso, D.; Kauffman, D. R. Electrochemical Carbon Dioxide Reduction at Nanostructured Gold, Copper, and Alloy Materials. Energy Technol. 2017, 5, 775-795.

(20) Pander, J. E.; Ren, D.; Huang, Y.; Loo, N. W. X.; Hong, S. H. L.; Yeo, B. S. Understanding the Heterogeneous Electrocatalytic Reduction of Carbon Dioxide on Oxide-Derived Catalysts. ChemElectroChem 2018, 5, 219-237.

(21) He, J.; Johnson, N. J. J.; Huang, A.; Berlinguette, C. P. Electrocatalytic Alloys for CO 2 Reduction. ChemSusChem 2018, 11, 48-57.

(22) Intergovernmental Panel on Climate Change. Climate Change 2014: Synthesis Report; 2014.

(23) Conference of the Parties to the United Nations Framework Convention on Climate Change. Adoption of the Paris Agreement; 2015; pp 1-32.

(24) Intergovernmental Panel on Climate Change. IPCC Special Report on the Impacts of Global Warming of $1.5^{\circ} \mathrm{C}$ - Summary for Policy Makers; Incheon, 2018.

(25) International Energy Agency. IEA2017.Pdf. IEA Newsroom. 2017.

(26) Obama, B. The Irreversible Momentum of Clean Energy. Science 2017, 355, 126-129. 
(27) World Bank Group: IEA Statistics. GDP per unit of energy use https://data.worldbank.org/indicator/EG.GDP.PUSE.KO.PP?contextual=default\&locations=U S-CN-EU-1W.

(28) Chu, S.; Cui, Y.; Liu, N. The Path towards Sustainable Energy. Nat. Mater. 2016, 16, 16-22.

(29) Randall, T. Wind and Solar Are Crushing Fossil Fuels. Bloomberg. 2016.

(30) Davis, S. J.; Lewis, N. S.; Shaner, M.; Aggarwal, S.; Arent, D.; Azevedo, I. L.; Benson, S. M.; Bradley, T.; Brouwer, J.; Chiang, Y.-M.; et al. Net-Zero Emissions Energy Systems. Science 2018, 360, eaas9793.

(31) Yang, Z.; Zhang, J.; Kintner-Meyer, M. C. W.; Lu, X.; Choi, D.; Lemmon, J. P.; Liu, J. Electrochemical Energy Storage for Green Grid. Chem. Rev. 2011, 111, 3577-3613.

(32) Gyuk, I.; Johnson, M.; Vetrano, J.; Lynn, K.; Parks, W.; Handa, R.; Kannberg, L. D.; Hearne, S.; Waldrip, K.; Braccio, R. Grid Energy Storage; 2013.

(33) van Oss, H. G. Cement in January 2018; 2018.

(34) World Steel Association. Worl Steel Figures 2018; Brussels and Beijing, 2018.

(35) Geyer, R.; Jambeck, J. R.; Law, K. L. Production, Use, and Fate of All Plastics Ever Made. Sci. Adv. 2017, 3, 19-24.

(36) Apodaca, L. E. Mineral Commodity Summaries - Ammonia; 2016; Vol. 32.

(37) Bray, E. L. Mineral Commodity Summaries - Aluminum; 2017; Vol. 1.

(38) Houghton, R. A. Balancing the Global Carbon Budget. Annu. Rev. Earth Planet. Sci. 2007, 35, 313-347.

(39) Le Quéré, C.; Moriarty, R.; Andrew, R. M.; Canadell, J. G.; Sitch, S.; Korsbakken, J. I.; Friedlingstein, P.; Peters, G. P.; Andres, R. J.; Boden, T. A.; et al. Global Carbon Budget 2015. Earth Syst. Sci. Data 2015, 7, 349-396.

(40) Majumdar, A.; Deutch, J.; Bras, R.; Benson, S.; Carter, E.; Ort, D.; Ramage, M.; Socolow, R.; Toone, E.; Whitesides, G.; et al. Task Force on the RD\&D Strategy for CO2 Utilization and/or Negative Emissions at the Gigatonne Scale; 2016.

(41) IPCC. IPCC Special Report on the Impacts of Global Warming of $1.5^{\circ} \mathrm{C}$ - Summary for Policy Makers. 2018.

(42) Global CCS Institute. The Global Status of CCS; 2013.

(43) North American Carbon Atlas Partnership. The North American Carbon Storage Atlas. 2012.

(44) Rubin, E. S.; Davison, J. E.; Herzog, H. J. The Cost of CO2 Capture and Storage. Int. J. Greenh. Gas Control 2015, 40, 378-400.

(45) Appel, A. M.; Bercaw, J. E.; Bocarsly, A. B.; Dobbek, H.; DuBois, D. L.; Dupuis, M.; Ferry, J. G.; Fujita, E.; Hille, R.; Kenis, Paul J, A.; et al. Frontiers, Opportunities, and Challenges in Biochemical and Chemical Catalysis of CO2 Fixation. Chem. Rev. 2013, 113, 6621-6658.

(46) Searchinger, T.; Heimlich, R.; Houghton, R. A.; Dong, F.; Elobeid, A.; Fabiosa, J.; Tokgoz, S.; Hayes, D.; Yu, T.-H. Use of U.S. Croplands for Biofuels Increases Greenhouse Gases Through Emissions from Land-Use Change. Science 2008, 319, 1238-1240.

(47) Al-Riffai, P.; Dimaranan, B.; Laborde, D. Global Trade and Environmental Impact Study of the EU Biofuels Mandate; 2010.

(48) Baik, E.; Sanchez, D. L.; Turner, P. A.; Mach, K. J.; Field, C. B.; Benson, S. M. Geospatial 
Analysis of Near-Term Potential for Carbon-Negative Bioenergy in the United States. Proc. Natl. Acad. Sci. 2018, 115, 3290-3295.

(49) Turner, P. A.; Mach, K. J.; Lobell, D. B.; Benson, S. M.; Baik, E.; Sanchez, D. L.; Field, C. B. The Global Overlap of Bioenergy and Carbon Sequestration Potential. Clim. Change 2018, $148,1-10$.

(50) Kaul, M.; Mohren, G. M. J.; Dadhwal, V. K. Carbon Storage and Sequestration Potential of Selected Tree Species in India. Mitig. Adapt. Strateg. Glob. Chang. 2010, 15, 489-510.

(51) Eckert, M.; Fleischmann, G.; Jira, R.; Bolt, H. M.; Golka, K. Ullmann's Encyclopedia of Industrial Chemistry; Wiley-VCH Verlag GmbH \& Co. KGaA: Weinheim, Germany, 2000.

(52) Chorkendorff, I.; Niemantsverdriet, J. W. Concepts of Modern Catalysis and Kinetics; WileyVCH Verlag GmbH \& Co. KGaA: Weinheim, FRG, 2003.

(53) Rönsch, S.; Schneider, J.; Matthischke, S.; Schlülter, M.; Götz, M.; Lefebvre, J.; Prabhakaran, P.; Bajohr, S. Review on Methanation - From Fundamentals to Current Projects. Fuel 2016, 166, 276-296.

(54) Yang, J.; Ma, W.; Chen, D.; Holmen, A.; Davis, B. H. Fischer-Tropsch Synthesis: A Review of the Effect of CO Conversion on Methane Selectivity. Appl. Catal. A Gen. 2014, 470, 250 260.

(55) Jadhav, S. G.; Vaidya, P. D.; Bhanage, B. M.; Joshi, J. B. Catalytic Carbon Dioxide Hydrogenation to Methanol: A Review of Recent Studies. Chem. Eng. Res. Des. 2014, 92, $2557-2567$.

(56) Olah, G. A.; Goeppert, A.; Prakash, G. K. S. Beyond Oil and Gas: The Methanol Economy: Second Edition; 2009.

(57) Studt, F.; Behrens, M.; Kunkes, E. L.; Thomas, N.; Zander, S.; Tarasov, A.; Schumann, J.; Frei, E.; Varley, J. B.; Abild-Pedersen, F.; et al. The Mechanism of CO and CO2 Hydrogenation to Methanol over Cu-Based Catalysts. ChemCatChem 2015, 7, 1105-1111.

(58) Seh, Z. W.; Kibsgaard, J.; Dickens, C. F.; Chorkendorff, I.; Nørskov, J. K.; Jaramillo, T. F. Combining Theory and Experiment in Electrocatalysis: Insights into Materials Design. Science 2017, 355, eaad4998.

(59) Carmo, M.; Fritz, D. L.; Mergel, J.; Stolten, D. A Comprehensive Review on PEM Water Electrolysis. Int. J. Hydrogen Energy 2013, 38, 4901-4934.

(60) Whipple, D. T.; Kenis, P. J. A. Prospects of CO2 Utilization via Direct Heterogeneous Electrochemical Reduction. J. Phys. Chem. Lett. 2010, 1, 3451-3458.

(61) Graves, C.; Ebbesen, S. D.; Mogensen, M.; Lackner, K. S. Sustainable Hydrocarbon Fuels by Recycling $\mathrm{CO} 2$ and $\mathrm{H} 2 \mathrm{O}$ with Renewable or Nuclear Energy. Renew. Sustain. Energy Rev. 2011, 15, 1-23.

(62) Kondratenko, E. V.; Mul, G.; Baltrusaitis, J.; Larrazábal, G. O.; Pérez-Ramírez, J. Status and Perspectives of $\mathrm{CO} 2$ Conversion into Fuels and Chemicals by Catalytic, Photocatalytic and Electrocatalytic Processes. Energy Environ. Sci. 2013, 6, 3112-3135.

(63) Götz, M.; Lefebvre, J.; Mörs, F.; McDaniel Koch, A.; Graf, F.; Bajohr, S.; Reimert, R.; Kolb, T. Renewable Power-to-Gas: A Technological and Economic Review. Renew. Energy 2016, 85, 1371-1390.

(64) Dau, H.; Limberg, C.; Reier, T.; Risch, M.; Roggan, S.; Strasser, P. The Mechanism of Water Oxidation: From Electrolysis via Homogeneous to Biological Catalysis. ChemCatChem 2010, $2,724-761$. 
(65) NIST Chemistry WebBook, NIST Standard Reference Database Number 69; Mallard, P. J., Linstrom, W. G., Eds.; National Institute of Standards and Technology: Gaithersburg MD, 20899.

(66) Kuhl, K. P.; Cave, E. R.; Abram, D. N.; Jaramillo, T. F. New Insights into the Electrochemical Reduction of Carbon Dioxide on Metallic Copper Surfaces. Energy Environ. Sci. 2012, 5 , 7050-7059.

(67) Verma, S.; Kim, B.; Jhong, H. R. M.; Ma, S.; Kenis, P. J. A. A Gross-Margin Model for Defining Technoeconomic Benchmarks in the Electroreduction of CO2. ChemSusChem 2016, 9, 1972-1979.

(68) Oloman, C.; Li, H. Electrochemical Processing of Carbon Dioxide. ChemSusChem 2008, 1, $385-391$.

(69) Spurgeon, J. M.; Kumar, B. A Comparative Technoeconomic Analysis of Pathways for Commercial Electrochemical CO2 Reduction to Liquid Products. Energy Environ. Sci. 2018, $11,1536-1551$.

(70) Jouny, M.; Luc, W.; Jiao, F. General Techno-Economic Analysis of CO2 Electrolysis Systems. Ind. Eng. Chem. Res. 2018, 57, 2165-2177.

(71) Li, C. W.; Ciston, J.; Kanan, M. W. Electroreduction of Carbon Monoxide to Liquid Fuel on Oxide-Derived Nanocrystalline Copper. Nature 2014, 508, 504-507.

(72) Bushuyev, O. S.; De Luna, P.; Dinh, C. T.; Tao, L.; Saur, G.; van de Lagemaat, J.; Kelley, S. O.; Sargent, E. H. What Should We Make with CO2 and How Can We Make It? Joule 2018, 2, $825-832$.

(73) Clark, E. L.; Hahn, C.; Jaramillo, T. F.; Bell, A. T. Electrochemical CO2 Reduction over Compressively Strained CuAg Surface Alloys with Enhanced Multi-Carbon Oxygenate Selectivity. J. Am. Chem. Soc. 2017, 139, 15848-15857.

(74) Lewis, N. S.; Nocera, D. G. Powering the Planet: Chemical Challenges in Solar Energy Utilization. Proc. Natl. Acad. Sci. 2006, 103, 15729-15735.

(75) Afshar, A. A. N. Chemical Profile: Formic Acid; 2014.

(76) British Petroleum (BP). Natural Gas: 67th Edition; 2018.

(77) True, W. R. Global Ethylene Capacity Poised for Major Expansion. Oil Gas J. 2008, 110, 9095.

(78) Eckert, M.; Fleischmann, G.; Jira, R.; Bolt, H. M.; Golka, K. Ullmann's Encyclopedia of Industrial Chemistry; WILEY-VCH Verlag, 2007.

(79) Kniel, L.; Stork, K.; Winter, O. Ethylene - A Keystone to the Petrochemical Industry; New York: M. Dekker, 1980.

(80) Mussatto, S. I.; Dragone, G.; Guimarães, P. M. R.; Silva, J. P. A.; Carneiro, L. M.; Roberto, I. C.; Vicente, A.; Domingues, L.; Teixeira, J. A. Technological Trends, Global Market, and Challenges of Bio-Ethanol Production. Biotechnol. Adv. 2010, 28, 817-830.

(81) Teeter, T. E.; Van Rysselberghe, P. Reduction of Carbon Dioxide on Mercury Cathodes. $J$. Chem. Phys. 1954, 22, 759-760.

(82) Hori, Y.; Kikuchi, K.; Suzuki, S. Production of CO and CH4 in Electrochemical Reduction of $\mathrm{CO} 2$ at Metal Electrodes in Aqueous Hydrogencarbonate Solutions. Chem. Lett. 1985, 14, $1695-1698$.

(83) Hori, Y.; Kikuchi, K.; Murata, A.; Suzuki, S. Production of Methane and Ethylene in 
Electrochemical Reduction of Carbon Dioxide At Copper Electrode in Aqueous Hydrogencarbonate Solution. Chem. Lett. 1986, 15, 897-898.

(84) Hori, Y.; Murata, A.; Takahashi, R.; Suzuki, S. Enhanced Formation of Ethylene and Alcohols at Ambient Temperature and Pressure in Electrochemical Reduction of Carbon Dioxide at a Copper Electrode. J. Chem. Soc. Chem. Commun. 1988, O, 17.

(85) Hori, Y.; Wakebe, H.; Tsukamoto, T.; Koga, O. Electrocatalytic Process of CO Selectivity in Electrochemical Reduction of $\mathrm{CO} 2$ at Metal Electrodes in Aqueous Media. Electrochim. Acta 1994, 39, 1833-1839.

(86) Hori, Y.; Murata, A.; Takahashi, R. Formation of Hydrocarbons in the Electrochemical Reduction of Carbon Dioxide at a Copper Electrode in Aqueous Solution. J. Chem. Soc. Faraday Trans. I 1989, 85, 2309-2326.

(87) Bagger, A.; Ju, W.; Varela, A. S.; Strasser, P.; Rossmeisl, J. Electrochemical CO2 Reduction: A Classification Problem. ChemPhysChem 2017, 18, 3266-3273.

(88) Clark, E. L.; Resasco, J.; Landers, A.; Lin, J.; Chung, L.-T.; Walton, A.; Hahn, C.; Jaramillo, T. F.; Bell, A. T. Standards and Protocols for Data Acquisition and Reporting for Studies of the Electrochemical Reduction of Carbon Dioxide. ACS Catal. 2018, 8, 6560-6570.

(89) Pander, J. E.; Ren, D.; Yeo, B. S. Practices for the Collection and Reporting of Electrocatalytic Performance and Mechanistic Information for the CO2 Reduction Reaction. Catal. Sci. Technol. 2017, 7, 5820-5832.

(90) Li, C. W.; Kanan, M. W. CO2 Reduction at Low Overpotential on Cu Electrodes Resulting from the Reduction of Thick Cu2O Films. J. Am. Chem. Soc. 2012, 134, 7231-7234.

(91) Bertheussen, E.; Verdaguer-Casadevall, A.; Ravasio, D.; Montoya, J. H.; Trimarco, D. B.; Roy, C.; Meier, S.; Wendland, J.; Nørskov, J. K.; Stephens, I. E. L.; et al. Acetaldehyde as an Intermediate in the Electroreduction of Carbon Monoxide to Ethanol on Oxide-Derived Copper. Angew. Chemie Int. Ed. 2016, 55, 1450-1454.

(92) Raciti, D.; Livi, K. J.; Wang, C. Highly Dense Cu Nanowires for Low-Overpotential CO2 Reduction. Nano Lett. 2015, 15, 6829-6835.

(93) Ma, M.; Djanashvili, K.; Smith, W. A. Selective Electrochemical Reduction of CO2 to CO on CuO-Derived Cu Nanowires. Phys. Chem. Chem. Phys. 2015, 17, 20861-20867.

(94) Min, S.; Yang, X.; Lu, A. Y.; Tseng, C. C.; Hedhili, M. N.; Li, L. J.; Huang, K. W. Low Overpotential and High Current CO2 Reduction with Surface Reconstructed Cu Foam Electrodes. Nano Energy 2016, 27, 121-129.

(95) Roberts, F. S.; Kuhl, K. P.; Nilsson, A. High Selectivity for Ethylene from Carbon Dioxide Reduction over Copper Nanocube Electrocatalysts. Angew. Chemie Int. Ed. 2015, 54, 51795182.

(96) Kwon, Y.; Lum, Y.; Clark, E. L.; Ager, J. W.; Bell, A. T. CO2 Electroreduction with Enhanced Ethylene and Ethanol Selectivity by Nanostructuring Polycrystalline Copper. ChemElectroChem 2016, 3, 1012-1019.

(97) Singh, M. R.; Clark, E. L.; Bell, A. T. Effects of Electrolyte, Catalyst, and Membrane Composition and Operating Conditions on the Performance of Solar-Driven Electrochemical Reduction of Carbon Dioxide. Phys. Chem. Chem. Phys. 2015, 17, 18924-18936.

(98) Lum, Y.; Yue, B.; Lobaccaro, P.; Bell, A. T.; Ager, J. W. Optimizing C-C Coupling on OxideDerived Copper Catalysts for Electrochemical CO2 Reduction. J. Phys. Chem. C 2017, 121, 14191-14203.

(99) Raciti, D.; Mao, M.; Wang, C. Mass Transport Modelling for the Electroreduction of CO2 on 
Cu Nanowires. Nanotechnology 2018, 29, 044001.

(100) Gupta, N.; Gattrell, M.; MacDougall, B. Calculation for the Cathode Surface Concentrations in the Electrochemical Reduction of $\mathrm{CO} 2$ in KHCO3 Solutions. J. Appl. Electrochem. 2006, 36, $161-172$.

(101) Clark, E. L.; Bell, A. T. Direct Observation of the Local Reaction Environment during the Electrochemical Reduction of CO2. J. Am. Chem. Soc. 2018, 140, 7012-7020.

(102) Hashiba, H.; Yotsuhashi, S.; Deguchi, M.; Yamada, Y. Systematic Analysis of Electrochemical CO2 Reduction with Various Reaction Parameters Using Combinatorial Reactors. ACS Comb. Sci. 2016, 18, 203-208.

(103) Hara, K.; Tsuneto, A.; Kudo, A.; Sakata, T. Electrochemical Reduction of CO2 on a Cu Electrode under High Pressure: Factors That Determine the Product Selectivity. J. Electrochem. Soc. 1994, 141, 2097-2103.

(104) Dewulf, D. W.; Jin, T.; Bard, A. J. Electrochemical and Surface Studies of Carbon Dioxide Reduction to Methane and Ethylene at Copper Electrodes in Aqueous Solutions. $J$. Electrochem. Soc. 1989, 136, 1686-1691.

(105) Wasmus, S.; Cattaneo, E.; Vielstich, W. Reduction of Carbon Dioxide to Methane and Ethene - An On-Line MS Study with Rotating Electrodes. Electrochim. Acta 1990, 35, 771-775.

(106) Yano, H.; Shirai, F.; Nakayama, M.; Ogura, K. Efficient Electrochemical Conversion of CO2 to $\mathrm{CO}, \mathrm{C} 2 \mathrm{H} 4$ and $\mathrm{CH} 4$ at a Three-Phase Interface on a $\mathrm{Cu}$ Net Electrode in Acidic Solution. $J$. Electroanal. Chem. 2002, 519, 93-100.

(107) Xie, J. F.; Huang, Y. X.; Li, W. W.; Song, X. N.; Xiong, L.; Yu, H. Q. Efficient Electrochemical CO2 Reduction on a Unique Chrysanthemum-like Cu Nanoflower Electrode and Direct Observation of Carbon Deposite. Electrochim. Acta 2014, 139, 137-144.

(108) Kyriacou, G.; Anagnostopoulos, A. Electroreduction of CO2 on Differently Prepared Copper Electrodes: The Influence of Electrode Treatment on the Current Efficiences. J. Electroanal. Chem. 1992, 322, 233-246.

(109) Jermann, B.; Augustynski, J. Long-Term Activation of the Copper Cathode in the Course of CO2 Reduction. Electrochim. Acta 1994, 39, 1891-1896.

(110) Kas, R.; Kortlever, R.; Y1lmaz, H.; Koper, M. T. M.; Mul, G. Manipulating the Hydrocarbon Selectivity of Copper Nanoparticles in $\mathrm{CO} 2$ Electroreduction by Process Conditions. ChemElectroChem 2015, 2, 354-358.

(111) Shiratsuchi, R.; Aikoh, Y.; Nogami, G. Pulsed Electroreduction of CO2 on Copper Electrodes. J. Electrochem. Soc. 1993, 140, 3479-3482.

(112) Hori, Y.; Konishi, H.; Futamura, T.; Murata, A.; Koga, O.; Sakurai, H.; Oguma, K. "Deactivation of Copper Electrode" in Electrochemical Reduction of CO2. Electrochim. Acta 2005, 50, 5354-5369.

(113) Schouten, K. J. P.; Kwon, Y.; van der Ham, C. J. M.; Qin, Z.; Koper, M. T. M. A New Mechanism for the Selectivity to C1 and C2 Species in the Electrochemical Reduction of Carbon Dioxide on Copper Electrodes. Chem. Sci. 2011, 2, 1902-1909.

(114) Wuttig, A.; Surendranath, Y. Impurity Ion Complexation Enhances Carbon Dioxide Reduction Catalysis. ACS Catal. 2015, 5, 4479-4484.

(115) Bertheussen, E.; Hogg, T. V.; Abghoui, Y.; Engstfeld, A. K.; Chorkendorff, I.; Stephens, I. E. L. Electroreduction of $\mathrm{CO}$ on Polycrystalline Copper at Low Overpotentials. ACS Energy Lett. 2018, 3, 634-640. 
(116) Kas, R.; Kortlever, R.; Milbrat, A.; Koper, M. T. M.; Mul, G.; Baltrusaitis, J. Electrochemical $\mathrm{CO} 2$ Reduction on Cu2O-Derived Copper Nanoparticles: Controlling the Catalytic Selectivity of Hydrocarbons. Phys. Chem. Chem. Phys. 2014, 16, 12194-12201.

(117) Chen, C. S.; Handoko, A. D.; Wan, J. H.; Ma, L.; Ren, D.; Yeo, B. S. Stable and Selective Electrochemical Reduction of Carbon Dioxide to Ethylene on Copper Mesocrystals. Catal. Sci. Technol. 2015, 5, 161-168.

(118) Lee, J.; Tak, Y. Electrocatalytic Activity of Cu Electrode in Electroreduction of CO2. Electrochim. Acta 2001, 46, 3015-3022.

(119) Dunwell, M.; Lu, Q.; Heyes, J. M.; Rosen, J.; Chen, J. G.; Yan, Y.; Jiao, F.; Xu, B. The Central Role of Bicarbonate in the Electrochemical Reduction of Carbon Dioxide on Gold. $J$. Am. Chem. Soc. 2017, 139, 3774-3783.

(120) Yano, J.; Yamasaki, S. Pulse-Mode Electrochemical Reduction of Carbon Dioxide Using Copper and Copper Oxide Electrodes for Selective Ethylene Formation. J. Appl. Electrochem. 2008, 38, 1721-1726.

(121) Kumar, B.; Brian, J. P.; Atla, V.; Kumari, S.; Bertram, K. A.; White, R. T.; Spurgeon, J. M. Controlling the Product Syngas H2:CO Ratio through Pulsed-Bias Electrochemical Reduction of $\mathrm{CO} 2$ on Copper. ACS Catal. 2016, 6, 4739-4745.

(122) Le Duff, C. S.; Lawrence, M. J.; Rodriguez, P. Role of the Adsorbed Oxygen Species in the Selective Electrochemical Reduction of $\mathrm{CO} 2$ to Alcohols and Carbonyls on Copper Electrodes. Angew. Chemie Int. Ed. 2017, 56, 12919-12924.

(123) Ishimaru, S.; Shiratsuchi, R.; Nogami, G. Pulsed Electroreduction of CO2 on Cu-Ag Alloy Electrodes. J. Electrochem. Soc. 2000, 147, 1864-1867.

(124) Kimura, K. W.; Fritz, K. E.; Kim, J.; Suntivich, J.; Abruña, H. D.; Hanrath, T. Controlled Selectivity of $\mathrm{CO} 2$ Reduction on Copper by Pulsing the Electrochemical Potential. ChemSusChem 2018, 11, 1781-1786.

(125) Eilert, A.; Roberts, F. S.; Friebel, D.; Nilsson, A. Formation of Copper Catalysts for CO2 Reduction with High Ethylene/Methane Product Ratio Investigated with In Situ X-Ray Absorption Spectroscopy. J. Phys. Chem. Lett. 2016, 7, 1466-1470.

(126) Mistry, H.; Varela, A. S.; Bonifacio, C. S.; Zegkinoglou, I.; Sinev, I.; Choi, Y.; Kisslinger, K.; Stach, E. A.; Yang, J. C.; Strasser, P.; et al. Highly Selective Plasma-Activated Copper Catalysts for Carbon Dioxide Reduction to Ethylene. Nat. Commun. 2016, 7, 1-8.

(127) De Luna, P.; Quintero-Bermudez, R.; Dinh, C.-T.; Ross, M. B.; Bushuyev, O. S.; Todorovic, P.; Regier, T.; Kelley, S. O.; Yang, P.; Sargent, E. H. Catalyst Electro-Redeposition Controls Morphology and Oxidation State for Selective Carbon Dioxide Reduction. Nat. Catal. 2018, 1, $103-110$.

(128) Lee, S.; Park, G.; Lee, J. Importance of Ag-Cu Biphasic Boundaries for Selective Electrochemical Reduction of CO2 to Ethanol. ACS Catal. 2017, 7, 8594-8604.

(129) Ren, D.; Deng, Y.; Handoko, A. D.; Chen, C. S.; Malkhandi, S.; Yeo, B. S. Selective Electrochemical Reduction of Carbon Dioxide to Ethylene and Ethanol on Copper(I) Oxide Catalysts. ACS Catal. 2015, 5, 2814-2821.

(130) Ren, D.; Ang, B. S.-H.; Yeo, B. S. Tuning the Selectivity of Carbon Dioxide Electroreduction toward Ethanol on Oxide-Derived CuxZn Catalysts. ACS Catal. 2016, 6, 8239-8247.

(131) Hoang, T. T. H.; Ma, S.; Gold, J. I.; Kenis, P. J. A.; Gewirth, A. A. Nanoporous Copper Films by Additive-Controlled Electrodeposition: CO2 Reduction Catalysis. ACS Catal. 2017, 7, 3313-3321. 
(132) Hoang, T. T. H.; Verma, S.; Ma, S.; Fister, T. T.; Timoshenko, J.; Frenkel, A. I.; Kenis, P. J. A.; Gewirth, A. A. Nanoporous Copper-Silver Alloys by Additive-Controlled Electrodeposition for the Selective Electroreduction of CO 2 to Ethylene and Ethanol. J. Am. Chem. Soc. 2018, 140, 5791-5797.

(133) Ross, M. B.; Dinh, C. T.; Li, Y.; Kim, D.; De Luna, P.; Sargent, E. H.; Yang, P. Tunable Cu Enrichment Enables Designer Syngas Electrosynthesis from CO2. J. Am. Chem. Soc. 2017, 139, 9359-9363.

(134) Gunathunge, C. M.; Li, X.; Li, J.; Hicks, R. P.; Ovalle, V. J.; Waegele, M. M. Spectroscopic Observation of Reversible Surface Reconstruction of Copper Electrodes under CO2 Reduction. J. Phys. Chem. C 2017, 121, 12337-12344.

(135) Chernyshova, I. V.; Somasundaran, P.; Ponnurangam, S. On the Origin of the Elusive First Intermediate of CO2 Electroreduction. Proc. Natl. Acad. Sci. 2018, 115, E9261-E9270.

(136) Mandal, L.; Yang, K. R.; Motapothula, M. R.; Ren, D.; Lobaccaro, P.; Patra, A.; Sherburne, M.; Batista, V. S.; Yeo, B. S.; Ager, J. W.; et al. Investigating the Role of Copper Oxide in Electrochemical CO2 Reduction in Real Time. ACS Appl. Mater. Interfaces 2018, 10, 85748584.

(137) Figueiredo, M. C.; Ledezma-Yanez, I.; Koper, M. T. M. In Situ Spectroscopic Study of CO2 Electroreduction at Copper Electrodes in Acetonitrile. ACS Catal. 2016, 6, 2382-2392.

(138) Gunathunge, C. M.; Ovalle, V. J.; Waegele, M. M. Probing Promoting Effects of Alkali Cations on the Reduction of $\mathrm{CO}$ at the Aqueous Electrolyte/Copper Interface. Phys. Chem. Chem. Phys. 2017, 19, 30166-30172.

(139) Wuttig, A.; Yaguchi, M.; Motobayashi, K.; Osawa, M.; Surendranath, Y. Inhibited Proton Transfer Enhances Au-Catalyzed CO2-to-Fuels Selectivity. Proc. Natl. Acad. Sci. 2016, 113, E4585-E4593.

(140) Zhu, S.; Jiang, B.; Cai, W.-B.; Shao, M. Direct Observation on Reaction Intermediates and the Role of Bicarbonate Anions in CO2 Electrochemical Reduction Reaction on Cu Surfaces. $J$. Am. Chem. Soc. 2017, 139, 15664-15667.

(141) Pérez-Gallent, E.; Figueiredo, M. C.; Calle-Vallejo, F.; Koper, M. T. M. Spectroscopic Observation of a Hydrogenated CO Dimer Intermediate During CO Reduction on $\mathrm{Cu}(100)$ Electrodes. Angew. Chemie Int. Ed. 2017, 56, 3621-3624.

(142) Baruch, M. F.; Pander, J. E.; White, J. L.; Bocarsly, A. B. Mechanistic Insights into the Reduction of CO2 on Tin Electrodes Using in Situ ATR-IR Spectroscopy. ACS Catal. 2015, 5, $3148-3156$.

(143) Pander, J. E.; Baruch, M. F.; Bocarsly, A. B. Probing the Mechanism of Aqueous CO2 Reduction on Post-Transition-Metal Electrodes Using ATR-IR Spectroelectrochemistry. ACS Catal. 2016, 6, 7824-7833.

(144) Phillips, K. R.; Katayama, Y.; Hwang, J.; Shao-Horn, Y. Sulfide-Derived Copper for Electrochemical Conversion of CO2 to Formic Acid. J. Phys. Chem. Lett. 2018, 9, 4407-4412.

(145) Hori, Y.; Murata, A.; Tsukamoto, T.; Wakebe, H.; Koga, O.; Yamazaki, H. Adsorption of Carbon Monoxide at a Copper Electrode Accompanied by Electron Transfer Observed by Voltammetry and IR Spectroscopy. Electrochim. Acta 1994, 39, 2495-2500.

(146) Hori, Y.; Koga, O.; Yamazaki, H.; Matsuo, T. Infrared Spectroscopy of Adsorbed CO and Intermediate Species in Electrochemical Reduction of $\mathrm{CO} 2$ to Hydrocarbons on a $\mathrm{Cu}$ Electrode. Electrochim. Acta 1995, 40, 2617-2622.

(147) Wuttig, A.; Liu, C.; Peng, Q.; Yaguchi, M.; Hendon, C. H.; Motobayashi, K.; Ye, S.; Osawa, 
M.; Surendranath, Y. Tracking a Common Surface-Bound Intermediate during CO2-to-Fuels Catalysis. ACS Cent. Sci. 2016, 2, 522-528.

(148) Gunathunge, C. M.; Ovalle, V. J.; Li, Y.; Janik, M. J.; Waegele, M. M. Existence of an Electrochemically Inert CO Population on Cu Electrodes in Alkaline PH. ACS Catal. 2018, 8, 7507-7516.

(149) Ayemoba, O.; Cuesta, A. Spectroscopic Evidence of Size-Dependent Buffering of Interfacial PH by Cation Hydrolysis during CO2 Electroreduction. ACS Appl. Mater. Interfaces 2017, 9, 27377-27382.

(150) Dunwell, M.; Yang, X.; Setzler, B. P.; Anibal, J.; Yan, Y.; Xu, B. Examination of NearElectrode Concentration Gradients and Kinetic Impacts on the Electrochemical Reduction of CO2 Using Surface-Enhanced Infrared Spectroscopy. ACS Catal. 2018, 8, 3999-4008.

(151) Hori, Y.; Wakebe, H.; Tsukamoto, T.; Koga, O. Adsorption of CO Accompanied with Simultaneous Charge Transfer on Copper Single Crystal Electrodes Related with Electrochemical Reduction of CO2 to Hydrocarbons. Surf. Sci. 1995, 335, 258-263.

(152) Hori, Y.; Takahashi, I.; Koga, O.; Hoshi, N. Selective Formation of C2 Compounds from Electrochemical Reduction of CO2 at a Series of Copper Single Crystal Electrodes. J. Phys. Chem. B 2002, 106, 15-17.

(153) Takahashi, I.; Koga, O.; Hoshi, N.; Hori, Y. Electrochemical Reduction of CO2 at Copper Single Crystal $\mathrm{Cu}(\mathrm{S})-[\mathrm{n}(111) \times(111)]$ and $\mathrm{Cu}(\mathrm{S})-[\mathrm{n}(110) \times(100)]$ Electrodes. J. Electroanal. Chem. 2002, 533, 135-143.

(154) Hori, Y.; Takahashi, I.; Koga, O.; Hoshi, N. Electrochemical Reduction of Carbon Dioxide at Various Series of Copper Single Crystal Electrodes. J. Mol. Catal. A Chem. 2003, 199, 39-47.

(155) Christophe, J.; Doneux, T.; Buess-Herman, C. Electroreduction of Carbon Dioxide on CopperBased Electrodes: Activity of Copper Single Crystals and Copper-Gold Alloys.

Electrocatalysis 2012, 3, 139-146.

(156) Huang, Y.; Handoko, A. D.; Hirunsit, P.; Yeo, B. S. Electrochemical Reduction of CO2 Using Copper Single-Crystal Surfaces: Effects of CO* Coverage on the Selective Formation of Ethylene. ACS Catal. 2017, 7, 1749-1756.

(157) Yoshihara, N.; Arita, M.; Noda, M. Electrochemical Reduction of Carbon Dioxide on SingleCrystal Copper Membrane. ECS Trans. 2015, 66, 83-89.

(158) Hahn, C.; Hatsukade, T.; Kim, Y. G.; Vailionis, A.; Baricuatro, J. H.; Higgins, D. C.; Nitopi, S. A.; Soriaga, M. P.; Jaramillo, T. F. Engineering Cu Surfaces for the Electrocatalytic Conversion of CO2: Controlling Selectivity toward Oxygenates and Hydrocarbons. Proc. Natl. Acad. Sci. U. S. A. 2017, 114, 5918-5923.

(159) Frese, K. W. Electrochemical Reduction of CO2 at Solid Electrodes; Elsevier: Amsterdam, 1993.

(160) Hori, Y.; Takahashi, R.; Yoshinami, Y.; Murata, A. Electrochemical Reduction of CO at a Copper Electrode. J. Phys. Chem. B 1997, 101, 7075-7081.

(161) Hori, Y.; Murata, A.; Takahashi, R.; Suzuki, S. Electroreduction of CO To CH4 and C2H4 At a Copper Electrode in Aqueous-Solutions At Ambient-Temperature and Pressure. J. Am. Chem. Soc. 1987, 109, 5022-5023.

(162) Kim, J. J.; Summers, D. P.; Frese Jr., K. W. Reduction of Carbon Dioxide and Carbon Monoxide to Methane on Copper Foil Electrodes. J. Electroanal. Chem. Interfacial Electrochem. 1988, 245, 223-244.

(163) Hori, Y.; Koga, O.; Watanabe, Y.; Matsuo, T. FTIR Measurements of Charge Displacement 
Adsorption of CO on Poly- and Single Crystal (100) of Cu Electrodes. Electrochim. Acta 1998, 44, 1389-1395.

(164) Schouten, K. J. P.; Qin, Z.; Pérez Gallent, E.; Koper, M. T. M. Two Pathways for the Formation of Ethylene in CO Reduction on Single-Crystal Copper Electrodes. J. Am. Chem. Soc. 2012, 134, 9864-9867.

(165) Schouten, K. J. P.; Gallent, E. P.; Koper, M. T. M. The Electrochemical Characterization of Copper Single-Crystal Electrodes in Alkaline Media. J. Electroanal. Chem. 2013, 699, 6-9.

(166) Schouten, K. J. P.; Pérez Gallent, E.; Koper, M. T. M. The Influence of PH on the Reduction of $\mathrm{CO}$ and $\mathrm{CO} 2$ to Hydrocarbons on Copper Electrodes. J. Electroanal. Chem. 2014, 716, $53-$ 57.

(167) Schouten, K. J. P.; Pérez Gallent, E.; Koper, M. T. M.; Schouten, K., Gallent, E., Koper, M. Structure Sensitivity of the Electrochemical Reduction of Carbon Monoxide on Copper Single Crystals. ACS Catal. 2013, 3, 1292-1295.

(168) Dahl, S.; Logadottir, A.; Egeberg, R. C.; Larsen, J. H.; Chorkendorff, I.; Törnqvist, E.; Nørskov, J. K. Role of Steps in N2 Activation on Ru(0001). Phys. Rev. Lett. 1999, 83, 1814 1817.

(169) Liu, X.; Xiao, J.; Peng, H.; Hong, X.; Chan, K.; Nørskov, J. K. Understanding Trends in Electrochemical Carbon Dioxide Reduction Rates. Nat. Commun. 2017, 8, 15438.

(170) Climent, V.; Feliu, J. M. Thirty Years of Platinum Single Crystal Electrochemistry. J. Solid State Electrochem. 2011, 15, 1297-1315.

(171) Gómez-Marín, A. M.; Feliu, J. M. Pt(111) Surface Disorder Kinetics in Perchloric Acid Solutions and the Influence of Specific Anion Adsorption. Electrochim. Acta 2012, 82, 558569.

(172) Engstfeld, A. K.; Maagaard, T.; Horch, S.; Chorkendorff, I.; Stephens, I. E. L. Polycrystalline and Single-Crystal $\mathrm{Cu}$ Electrodes: Influence of Experimental Conditions on the Electrochemical Properties in Alkaline Media. Chem. - A Eur. J. 2018, 24, 17743-17755.

(173) Schlaup, C.; Horch, S. In-Situ STM Study of Phosphate Adsorption on Cu(111), Au(111) and $\mathrm{Cu} / \mathrm{Au}(111)$ Electrodes. Surf. Sci. 2013, 608, 44-54.

(174) Kim, Y.-G.; Javier, A.; Baricuatro, J. H.; Soriaga, M. P. Regulating the Product Distribution of $\mathrm{CO}$ Reduction by the Atomic-Level Structural Modification of the $\mathrm{Cu}$ Electrode Surface. Electrocatalysis 2016, 7, 391-399.

(175) Eren, B.; Zherebetskyy, D.; Patera, L. L.; Wu, C. H.; Somorjai, G. A.; Salmeron, M. Activation of $\mathrm{Cu}(111)$ Surface by Decomposition into Nanoclusters Driven by CO Adsorption. Science 2016, 351, 475-478.

(176) Tang, M. T.; Ulissi, Z. W.; Chan, K. Theoretical Investigations of Transition Metal Surface Energies under Lattice Strain and CO Environment. J. Phys. Chem. C 2018, 122, 1448114487.

(177) Scott, S. B.; Hogg, T. V; Landers, A. T.; Maagaard, T.; Bertheussen, E.; Lin, J. C.; Davis, R. C.; Beeman, J. W.; Higgins, D.; Drisdell, W. S.; et al. Absence of Oxidized Phases in Cu under CO Reduction Conditions. ACS Energy Lett. 2019, 4, 803-804.

(178) Hori, Y.; Murata, A.; Yoshinami, Y. Adsorption of CO, Intermediately Formed in Electrochemical Reduction of CO2, at a Copper Electrode. J. Chem. Soc. Faraday Trans. 1991, 87, 125-128.

(179) Kim, Y. G.; Baricuatro, J. H.; Javier, A.; Gregoire, J. M.; Soriaga, M. P. The Evolution of the Polycrystalline Copper Surface, First to $\mathrm{Cu}(111)$ and Then to $\mathrm{Cu}(100)$, at a Fixed CO2RR 
Potential: A Study by Operando EC-STM. Langmuir 2014, 30, 15053-15056.

(180) Kim, Y. G.; Soriaga, M. P. Cathodic Regeneration of a Clean and Ordered Cu(100)-(1×1) Surface from an Air-Oxidized and Disordered Electrode: An Operando STM Study. $J$. Electroanal. Chem. 2014, 734, 7-9.

(181) Kim, Y. G.; Javier, A.; Baricuatro, J. H.; Torelli, D.; Cummins, K. D.; Tsang, C. F.; Hemminger, J. C.; Soriaga, M. P. Surface Reconstruction of Pure-Cu Single-Crystal Electrodes under CO-Reduction Potentials in Alkaline Solutions: A Study by Seriatim ECSTM-DEMS. J. Electroanal. Chem. 2016, 780, 290-295.

(182) Kim, Y.; Baricuatro, J. H.; Soriaga, M. P. Surface Reconstruction of Polycrystalline Cu Electrodes in Aqueous KHCO3 Electrolyte at Potentials in the Early Stages of CO2 Reduction. Electrocatalysis 2018, 9, 526-530.

(183) Gasteiger, H. A.; Kocha, S. S.; Sompalli, B.; Wagner, F. T. Activity Benchmarks and Requirements for Pt, Pt-Alloy, and Non-Pt Oxygen Reduction Catalysts for PEMFCs. Appl. Catal. B Environ. 2005, 56, 9-35.

(184) Droog, J. M. M.; Schlenter, B. Oxygen Electrosorption on Copper Single Crystal Electrodes in Sodium Hydroxide Solution. J. Electroanal. Chem. Interfacial Electrochem. 1980, 112, 387390.

(185) Jović, V. D.; Jović, B. M. Surface Reconstruction during the Adsorption/Desorption of OH Species onto $\mathrm{Cu}(111)$ and $\mathrm{Cu}(100)$ in $0.1 \mathrm{M} \mathrm{NaOH}$ Solution. J. Serbian Chem. Soc. 2002, 67, $531-546$.

(186) Jovic, V. D.; Jovic, B. M. EIS and Differential Capacitance Measurements onto Single Crystal Faces in Different Solutions Part II : $\mathrm{Cu}$ ( 111 ) and $\mathrm{Cu}(100)$ in $0.1 \mathrm{M} \mathrm{NaOH} . J$. Electroanal. Chem. 2003, 541, 13-21.

(187) Matsuoka, O.; Ono, S. S.; Nozoye, H.; Yamamoto, S. Structure and Dynamics of OxyOverlayer on $\mathrm{Cu}\left(\begin{array}{lll}1 & 1 & 1\end{array}\right)$ Electrode Surfaces in Alkaline Aqueous Solution Revealed by Electrochemical STM and Quartz Crystal Microbalance Measurement. Surf. Sci. 2003, 545, 8 18.

(188) Bockris, J. O.; Pentland, N. The Mechanism of Hydrogen Evolution at Copper Cathodes in Aqueous Solutions. Trans. Faraday Soc. 1952, 48, 833-839.

(189) Pentland, N.; Bockris, J. O.; Sheldon, E. Hydrogen Evolution Reaction on Copper, Gold, Molybdenum, Palladium, Rhodium, and Iron. J. Electrochem. Soc. 1957, 104, 182-194.

(190) Sharifi-Asl, S.; Macdonald, D. D. Investigation of the Kinetics and Mechanism of the Hydrogen Evolution Reaction on Copper. J. Electrochem. Soc. 2013, 160, H382-H391.

(191) Łukomska, A.; Sobkowski, J. Potential of Zero Charge of Monocrystalline Copper Electrodes in Perchlorate Solutions. J. Electroanal. Chem. 2004, 567, 95-102.

(192) Siegenthaler, H.; Jüttner, K. Voltammetric Investigation of Lead Adsorption on $\mathrm{Cu}(111)$ Single Crystal Substrates. J. Electroanal. Chem. 1984, 163, 327-343.

(193) Creus, A. H.; Souto, R. M.; Gonz, S.; Laz, M. M.; Salvarezza, R. C.; Arvia, A. J. The Electrochemical Faceting of Copper in $85 \%$ Aqueous O-Phosphoric Acid by Using a Potential Reversal Technique. Appl. Surf. Sci. 1994, 81, 387-398.

(194) Burke, L. D.; Collins, J. A.; Horgan, M. A.; Hurley, L. M.; O’Mullane, A. P. Importance of the Active States of Surface Atoms with Regard to the Electrocatalytic Behaviour of Metal Electrodes in Aqueous Media. Electrochim. Acta 2000, 45, 4127-4134.

(195) Hampson, N. A.; Latham, R. J.; Lee, J. B.; Macdonald, K. I. Oxidations at Copper Electrodes: Part I. The Differential Capacitance of Polycrystalline Copper in Alkali. J. Electroanal. Chem. 
Interfacial Electrochem. 1971, 31, 57-62.

(196) Nakahara, S.; Okinaka, Y. The Hydrogen Effect in Copper. Mater. Sci. Eng. A 1988, 101, 227-230.

(197) Rosca, V.; Duca, M.; de Groot, M. T.; Koper, M. T. M. Nitrogen Cycle Electrocatalysis. Chem. Rev. 2009, 109, 2209-2244.

(198) Duca, M.; Koper, M. T. M. Powering Denitrification: The Perspectives of Electrocatalytic Nitrate Reduction. Energy Environ. Sci. 2012, 5, 9726-9742.

(199) Vvedenskii, A.; Grushevskaya, S.; Ganzha, S.; Eliseev, D.; Abakumova, L. I. Copper Oxides: Kinetics of Formation and Semiconducting Properties. Part II. Copper Single Crystals. J. Solid State Electrochem. 2014, 18, 3437-3451.

(200) Strehblow, H. H. Passivity of Metals Studied by Surface Analytical Methods, a Review. Electrochim. Acta 2016, 212, 630-648.

(201) Wang, L.; Nitopi, S. A.; Bertheussen, E.; Orazov, M.; Morales-Guio, C. G.; Liu, X.; Higgins, D. C.; Chan, K.; Nørskov, J. K.; Hahn, C.; et al. Electrochemical Carbon Monoxide Reduction on Polycrystalline Copper: Effects of Potential, Pressure, and PH on Selectivity toward Multicarbon and Oxygenated Products. ACS Catal. 2018, 8, 7445-7454.

(202) Peterson, A. A.; Abild-Pedersen, F.; Studt, F.; Rossmeisl, J.; Nørskov, J. K. How Copper Catalyzes the Electroreduction of Carbon Dioxide into Hydrocarbon Fuels. Energy Environ. Sci. 2010, 3, 1311-1315.

(203) Peterson, A. A.; Nørskov, J. K. Activity Descriptors for CO2 Electroreduction to Methane on Transition-Metal Catalysts. J. Phys. Chem. Lett. 2012, 3, 251-258.

(204) Kuhl, K. P.; Hatsukade, T.; Cave, E. R.; Abram, D. N.; Kibsgaard, J.; Jaramillo, T. F. Electrocatalytic Conversion of Carbon Dioxide to Methane and Methanol on Transition Metal Surfaces. J. Am. Chem. Soc. 2014, 136, 14107-14113.

(205) Sabatier, P. Hydrogenations et Deshydrogenations Par Catalyse. Berichte der Dtsch. Chem. Gesellschaft 1911, 44, 1984-2001.

(206) Ooka, H.; Figueiredo, M. C.; Koper, M. T. M. M. Competition between Hydrogen Evolution and Carbon Dioxide Reduction on Copper Electrodes in Mildly Acidic Media. Langmuir 2017, 33, 9307-9313.

(207) Cook, R. L.; MacDuff, R. C.; Sammells, A. F. Evidence for Formaldehyde, Formic Acid, and Acetaldehyde as Possible Intermediates during Electrochemical Carbon Dioxide Reduction at Copper. J. Electrochem. Soc. 1989, 136, 1982-1984.

(208) Garza, A.; Bell, A. T.; Head-Gordon, M. On the Mechanism of CO2 Reduction at Copper Surfaces: Pathways to C2 Products. ACS Catal. 2018, 8, 1490-1499.

(209) Resasco, J.; Chen, L. D.; Clark, E.; Tsai, C.; Hahn, C.; Jaramillo, T. F.; Chan, K.; Bell, A. T. Promoter Effects of Alkali Metal Cations on the Electrochemical Reduction of Carbon Dioxide. J. Am. Chem. Soc. 2017, 139, 11277-11287.

(210) Ledezma-Yanez, I.; Gallent, E. P.; Koper, M. T. M.; Calle-Vallejo, F. Structure-Sensitive Electroreduction of Acetaldehyde to Ethanol on Copper and Its Mechanistic Implications for CO and CO2 Reduction. Catal. Today 2016, 262, 90-94.

(211) Lum, Y.; Cheng, T.; Goddard, W. A.; Ager, J. W. Electrochemical CO Reduction Builds Solvent Water into Oxygenate Products. J. Am. Chem. Soc. 2018, 140, 9337-9340.

(212) Birdja, Y. Y.; Koper, M. T. M. The Importance of Cannizzaro-Type Reactions during Electrocatalytic Reduction of Carbon Dioxide. J. Am. Chem. Soc. 2017, 139, 2030-2034. 
(213) Liu, X.; Schlexer, P.; Xiao, J.; Ji, Y.; Wang, L.; Sandberg, R. B.; Tang, M.; Brown, K. S.; Peng, H.; Ringe, S.; et al. PH Effects on the Electrochemical Reduction of $\mathrm{CO}(2)$ towards $\mathrm{C} 2$ Products on Stepped Copper. Nat. Commun. 2019, 10, 32.

(214) Pinsent, B. R. W.; Pearson, L.; Roughton, F. J. W. The Kinetics of Combination of Carbon Dioxide with Hydroxide Ions. Trans. Faraday Soc. 1956, 1, 1512-1520.

(215) Singh, M. R.; Kwon, Y.; Lum, Y.; Ager, J. W.; Bell, A. T. Hydrolysis of Electrolyte Cations Enhances the Electrochemical Reduction of $\mathrm{CO} 2$ over $\mathrm{Ag}$ and Cu. J. Am. Chem. Soc. 2016, $138,13006-13012$.

(216) Handoko, A. D.; Ong, C. W.; Huang, Y.; Lee, Z. G.; Lin, L.; Panetti, G. B.; Yeo, B. S. Mechanistic Insights into the Selective Electroreduction of Carbon Dioxide to Ethylene on Cu2O-Derived Copper Catalysts. J. Phys. Chem. C 2016, 120, 20058-20067.

(217) Lim, C. F. C.; Harrington, D. A.; Marshall, A. T. Effects of Mass Transfer on the Electrocatalytic CO2 Reduction on Cu. Electrochim. Acta 2017, 238, 56-63.

(218) Resasco, J.; Lum, Y.; Clark, E.; Zeledon, J. Z.; Bell, A. T. Effects of Anion Identity and Concentration on Electrochemical Reduction of CO2. ChemElectroChem 2018, 5, 1064-1072.

(219) Strmcnik, D.; Uchimura, M.; Wang, C.; Subbaraman, R.; Danilovic, N.; Vliet, D. van der; Paulikas, A. P.; Stamenkovic, V. R.; Markovic, N. M. Improving the Hydrogen Oxidation Reaction Rate by Promotion of Hydroxyl Adsorption. Nat. Chem. 2013, 5, 300-306.

(220) Strmcnik, D.; Lopes, P. P.; Genorio, B.; Stamenkovic, V. R.; Markovic, N. M. Design Principles for Hydrogen Evolution Reaction Catalyst Materials. Nano Energy 2016, 29, 29-36.

(221) Grozovski, V.; Vesztergom, S.; Láng, G. G.; Broekmann, P. Electrochemical Hydrogen Evolution: H+ or H2O Reduction? A Rotating Disk Electrode Study. J. Electrochem. Soc. 2017, 164, E3171-E3178.

(222) Hall, A. S.; Yoon, Y.; Wuttig, A.; Surendranath, Y. Mesostructure-Induced Selectivity in CO2 Reduction Catalysis. J. Am. Chem. Soc. 2015, 137, 14834-14837.

(223) Wuttig, A.; Yoon, Y.; Ryu, J.; Surendranath, Y. Bicarbonate Is Not a General Acid in AuCatalyzed CO2 Electroreduction. J. Am. Chem. Soc. 2017, 139, 17109-17113.

(224) Yoon, Y.; Hall, A. S.; Surendranath, Y. Tuning of Silver Catalyst Mesostructure Promotes Selective Carbon Dioxide Conversion into Fuels. Angew. Chemie - Int. Ed. 2016, 55, 1528215286.

(225) Murata, A.; Hori, Y. Product Selectivity Affected by Cationic Species in Electrochemical Reduction of CO2 and CO at a Cu Electrode. Bull. Chem. Soc. Jpn. 1991, 64, 123-127.

(226) Kyriacou, G. Z.; Anagnostopoulos, A. K. Influence of CO2 Partial Pressure and the Supporting Electrolyte Cation on the Product Distribution in CO2 Electroreduction. J. Appl. Electrochem. 1993, 23, 483-486.

(227) Pérez-Gallent, E.; Marcandalli, G.; Figueiredo, M. C.; Calle-Vallejo, F.; Koper, M. T. M. Structure- and Potential-Dependent Cation Effects on CO Reduction at Copper Single-Crystal Electrodes. J. Am. Chem. Soc. 2017, 139, 16412-16419.

(228) Frumkin, A. N. Influence of Cation Adsorption on the Kinetics of Electrode Processes. Trans. Faraday Soc. 1959, 55, 156-167.

(229) Mills, J. N.; McCrum, I. T.; Janik, M. J. Alkali Cation Specific Adsorption onto Fcc(111) Transition Metal Electrodes. Phys. Chem. Chem. Phys. 2014, 16, 13699-13707.

(230) Strmcnik, D.; Kodama, K.; Van Der Vliet, D.; Greeley, J.; Stamenkovic, V. R.; Marković, N. M. The Role of Non-Covalent Interactions in Electrocatalytic Fuel-Cell Reactions on 
Platinum. Nat. Chem. 2009, 1, 466-472.

(231) Strmenik, D.; van der Vliet, D. F.; Chang, K.-C.; Komanicky, V.; Kodama, K.; You, H.; Stamenkovic, V. R.; Marković, N. M. Effects of Li+, K+, and Ba2+ Cations on the ORR at Model and High Surface Area Pt and Au Surfaces in Alkaline Solutions. J. Phys. Chem. Lett. 2011, 2, 2733-2736.

(232) Akhade, S. A.; McCrum, I. T.; Janik, M. J. The Impact of Specifically Adsorbed Ions on the Copper-Catalyzed Electroreduction of CO2. J. Electrochem. Soc. 2016, 163, F477-F484.

(233) Chen, L. D.; Urushihara, M.; Chan, K.; Nørskov, J. K. Electric Field Effects in Electrochemical CO2 Reduction. ACS Catal. 2016, 6, 7133-7139.

(234) Montoya, J. H.; Shi, C.; Chan, K.; Nørskov, J. K. Theoretical Insights into a CO Dimerization Mechanism in CO2 Electroreduction. J. Phys. Chem. Lett. 2015, 6, 2032-2037.

(235) Thorson, M. R.; Siil, K. I.; Kenis, P. J. A. Effect of Cations on the Electrochemical Conversion of CO2 to CO. J. Electrochem. Soc. 2013, 160, F69-F74.

(236) Schizodimou, A.; Kyriacou, G. Acceleration of the Reduction of Carbon Dioxide in the Presence of Multivalent Cations. Electrochim. Acta 2012, 78, 171-176.

(237) Rosen, B. A.; Salehi-Khojin, A.; Thorson, M. R.; Zhu, W.; Whipple, D. T.; Kenis, P. J.; Masel, R. I. Ionic Liquid-Mediated Selective Conversion of $\mathrm{CO} 2$ to $\mathrm{CO}$ at Low Overpotentials. Science 2011, 334, 643-644.

(238) Rosen, B. A.; Haan, J. L.; Mukherjee, P.; Braunschweig, B.; Zhu, W.; Salehi-Khojin, A.; Dlott, D. D.; Masel, R. I. In Situ Spectroscopic Examination of a Low Overpotential Pathway for Carbon Dioxide Conversion to Carbon Monoxide. J. Phys. Chem. C 2012, 116, 1530715312.

(239) Asadi, M.; Kumar, B.; Behranginia, A.; Rosen, B. A.; Baskin, A.; Repnin, N.; Pisasale, D.; Phillips, P.; Zhu, W.; Haasch, R.; et al. Robust Carbon Dioxide Reduction on Molybdenum Disulphide Edges. Nat. Commun. 2014, 5, 4470.

(240) Feaster, J. T.; Jongerius, A. L.; Liu, X.; Urushihara, M.; Nitopi, S. A.; Hahn, C.; Chan, K.; Nørskov, J. K.; Jaramillo, T. F. Understanding the Influence of [EMIM]Cl on the Suppression of the Hydrogen Evolution Reaction on Transition Metal Electrodes. Langmuir 2017, 33, 9464-9471.

(241) Neubauer, S. S.; Schmid, B.; Reller, C.; Guldi, D. M.; Schmid, G. Alkalinity Initiated Decomposition of Mediating Imidazolium Ions in High Current Density CO2 Electrolysis. ChemElectroChem 2017, 4, 160-167.

(242) Varela, A. S.; Kroschel, M.; Reier, T.; Strasser, P. Controlling the Selectivity of CO2 Electroreduction on Copper: The Effect of the Electrolyte Concentration and the Importance of the Local PH. Catal. Today 2016, 260, 8-13.

(243) Ogura, K.; Oohara, R.; Kudo, Y. Reduction of CO2 to Ethylene at Three-Phase Interface Effects of Electrode Substrate and Catalytic Coating. J. Electrochem. Soc. 2005, 152, D213D219.

(244) Yano, H.; Tanaka, T.; Nakayama, M.; Ogura, K. Selective Electrochemical Reduction of CO2 to Ethylene at a Three-Phase Interface on Copper(I) Halide-Confined Cu-Mesh Electrodes in Acidic Solutions of Potassium Halides. J. Electroanal. Chem. 2004, 565, 287-293.

(245) Ogura, K.; Ferrell, J. R.; Cugini, A. V.; Smotkin, E. S.; Salazar-Villalpando, M. D. CO2 Attraction by Specifically Adsorbed Anions and Subsequent Accelerated Electrochemical Reduction. Electrochim. Acta 2010, 56, 381-386.

(246) Ogura, K.; Salazar-Villalpando, M. D. CO2 Electrochemical Reduction via Adsorbed Halide 
Anions. Jom 2011, 63, 35-38.

(247) McCrum, I. T.; Akhade, S. A.; Janik, M. J. Electrochemical Specific Adsorption of Halides on $\mathrm{Cu}$ 111,100, and 211: A Density Functional Theory Study. Electrochim. Acta 2015, 173, 302309.

(248) Varela, A. S.; Ju, W.; Reier, T.; Strasser, P. Tuning the Catalytic Activity and Selectivity of Cu for CO2 Electroreduction in the Presence of Halides. ACS Catal. 2016, 6, 2136-2144.

(249) Gao, D.; Scholten, F.; Roldan Cuenya, B. Improved CO2 Electroreduction Performance on Plasma-Activated Cu Catalysts via Electrolyte Design: Halide Effect. ACS Catal. 2017, 7, $5112-5120$.

(250) Lee, S.; Kim, D.; Lee, J. Electrocatalytic Production of C3-C4 Compounds by Conversion of $\mathrm{CO} 2$ on a Chloride-Induced Bi-Phasic Cu2O-Cu Catalyst. Angew. Chemie Int. Ed. 2015, 54, 14701-14705.

(251) Lobaccaro, P.; Singh, M. R.; Clark, E. L.; Kwon, Y.; Bell, A. T.; Ager, J. W. Effects of Temperature and Gas-liquid Mass Transfer on the Operation of Small Electrochemical Cells for the Quantitative Evaluation of CO2 Reduction Electrocatalysts. Phys. Chem. Chem. Phys. 2016, 18, 26777-26785.

(252) Kaneco, S.; Hiei, N.; Xing, Y.; Katsumata, H.; Ohnishi, H.; Suzuki, T.; Ohta, K. Electrochemical Conversion of Carbon Dioxide to Methane in Aqueous NaHCO3 Solution at Less than 273 K. Electrochem. Acta 2002, 48, 51-55.

(253) Kaneco, S.; Hiei, N.; Xing, Y.; Katsumata, H.; Ohnishi, H.; Suzuki, T.; Ohta, K. HighEfficiency Electrochemical CO2-to-Methane Reduction Method Using Aqueous KHCO3 Media at Less than 273 K. J. Solid State Electrochem. 2003, 7, 152-156.

(254) Azuma, M.; Hashimoto, K.; Watanabe, M. Electrochemical Reduction of Carbon Dioxide on Various Metal Electrodes in Low-Temperature Aqueous KHC03 Media. J. Electrochem. Soc. 1990, 137, 1772-1778.

(255) Ahn, S. T.; Abu-Baker, I.; Palmore, G. T. R. Electroreduction of CO2 on Polycrystalline Copper: Effect of Temperature on Product Selectivity. Catal. Today 2017, 288, 24-29.

(256) Hatsukade, T. Investigating the Effects of Chemical Composition and Surface Structure of Catalysts on the Electrochemical Reduction of Carbon Dioxide, Stanford University, 2016.

(257) Nørskov, J. K.; Rossmeisl, J.; Logadottir, A.; Lindqvist, L.; Kitchin, J. R.; Bligaard, T.; Jónsson, H. Origin of the Overpotential for Oxygen Reduction at a Fuel-Cell Cathode. J. Phys. Chem. B 2004, 108, 17886-17892.

(258) Karlberg, G. S.; Rossmeisl, J.; Nørskov, J. K. Estimations of Electric Field Effects on the Oxygen Reduction Reaction Based on the Density Functional Theory. Phys. Chem. Chem. Phys. 2007, 9, 5158-5161.

(259) Karlberg, G. S.; Jaramillo, T. F.; Skúlason, E.; Rossmeisl, J.; Bligaard, T.; Nørskov, J. K. Cyclic Voltammograms for $\mathrm{H}$ on $\mathrm{Pt}(111)$ and $\mathrm{Pt}(100)$ from First Principles. Phys. Rev. Lett. 2007, 99, 126101.

(260) Cheng, T.; Xiao, H.; Goddard, W. A. Full Atomistic Reaction Mechanism with Kinetics for $\mathrm{CO}$ Reduction on $\mathrm{Cu}(100)$ from Ab Initio Molecular Dynamics Free-Energy Calculations at 298 K. Proc. Natl. Acad. Sci. 2017, 114, 1795-1800.

(261) Goodpaster, J. D.; Bell, A. T.; Head-Gordon, M. Identification of Possible Pathways for C-C Bond Formation during Electrochemical Reduction of CO2: New Theoretical Insights from an Improved Electrochemical Model. J. Phys. Chem. Lett. 2016, 7, 1471-1477.

(262) Xiao, H.; Cheng, T.; Goddard, W. A.; Sundararaman, R. Mechanistic Explanation of the PH 
Dependence and Onset Potentials for Hydrocarbon Products from Electrochemical Reduction of $\mathrm{CO}$ on $\mathrm{Cu}$ (111). J. Am. Chem. Soc. 2016, 138, 483-486.

(263) Nie, X.; Luo, W.; Janik, M. J.; Asthagiri, A. Reaction Mechanisms of CO2 Electrochemical Reduction on $\mathrm{Cu}(111)$ Determined with Density Functional Theory. J. Catal. 2014, 312, 108 122.

(264) Nie, X.; Esopi, M. R.; Janik, M. J.; Asthagiri, A. Selectivity of CO2 Reduction on Copper Electrodes: The Role of the Kinetics of Elementary Steps. Angew. Chemie Int. Ed. 2013, 52, 2459-2462.

(265) Mathew, K.; Sundararaman, R.; Letchworth-Weaver, K.; Arias, T. A.; Hennig, R. G. Implicit Solvation Model for Density-Functional Study of Nanocrystal Surfaces and Reaction Pathways. J. Chem. Phys. 2014, 140, 084106.

(266) Schmickler, W.; Santos, E. Interfacial Electrochemistry; Springer Berlin Heidelberg, 2010.

(267) Mathew, K.; Hennig, R. Implicit Self-Consistent Description of Electrolyte in Plane-Wave Density-Functional Theory. 2016.

(268) Grün, F.; Jardat, M.; Turq, P.; Amatore, C. Relaxation of the Electrical Double Layer after an Electron Transfer Approached by Brownian Dynamics Simulation. J. Chem. Phys. 2004, 120, 9648-9655.

(269) Kling, M. F.; Vrakking, M. J. J. Attosecond Electron Dynamics. Annu. Rev. Phys. Chem. 2008, $59,463-492$.

(270) Chan, K.; Nørskov, J. K. Electrochemical Barriers Made Simple. J. Phys. Chem. Lett. 2015, 6, 2663-2668.

(271) Chan, K.; Nørskov, J. K. Potential Dependence of Electrochemical Barriers from Ab Initio Calculations. J. Phys. Chem. Lett. 2016, 7, 1686-1690.

(272) Björketun, M. E.; Zeng, Z.; Ahmed, R.; Tripkovic, V.; Thygesen, K. S.; Rossmeisl, J. Avoiding Pitfalls in the Modeling of Electrochemical Interfaces. Chem. Phys. Lett. 2013, 555, $145-148$.

(273) Chen, L. D.; Bajdich, M.; Martirez, J. M. P.; Krauter, C. M.; Gauthier, J. A.; Carter, E. A.; Luntz, A. C.; Chan, K.; Nørskov, J. K. Understanding the Apparent Fractional Charge of Protons in the Aqueous Electrochemical Double Layer. Nat. Commun. 2018, 9, 1-8.

(274) Shi, C.; Chan, K.; Yoo, J. S.; Nørskov, J. K. Barriers of Electrochemical CO2 Reduction on Transition Metals. Org. Process Res. Dev. 2016, 20, 1424-1430.

(275) Cheng, T.; Xiao, H.; Goddard, W. A. Free-Energy Barriers and Reaction Mechanisms for the Electrochemical Reduction of $\mathrm{CO}$ on the $\mathrm{Cu}(100)$ Surface, Including Multiple Layers of Explicit Solvent at PH 0. J. Phys. Chem. Lett. 2015, 6, 4767-4773.

(276) Luo, W.; Nie, X.; Janik, M. J.; Asthagiri, A. Facet Dependence of CO2 Reduction Paths on Cu Electrodes. ACS Catal. 2016, 6, 219-229.

(277) Shi, C.; Hansen, H. A.; Lausche, A. C.; Nørskov, J. K. Trends in Electrochemical CO2 Reduction Activity for Open and Close-Packed Metal Surfaces. Phys. Chem. Chem. Phys. 2014, 16, 4720-4727.

(278) Nørskov, J. K.; Studt, F.; Abild-Pedersen, F.; Bligaard, T. Fundamental Concepts in Heterogeneous Catalysis; John Wiley \& Sons, Inc: Hoboken, NJ, USA, 2014.

(279) Cheng, T.; Xiao, H.; Goddard, W. A. Reaction Mechanisms for the Electrochemical Reduction of CO2 to $\mathrm{CO}$ and Formate on the $\mathrm{Cu}(100)$ Surface at $298 \mathrm{~K}$ from Quantum Mechanics Free Energy Calculations with Explicit Water. J. Am. Chem. Soc. 2016, 138, 13802-13805. 
(280) Nie, X.; Griffin, G. L.; Janik, M. J.; Asthagiri, A. Surface Phases of Cu2O(111) under CO2 Electrochemical Reduction Conditions. Catal. Commun. 2014, 52, 88-91.

(281) Montoya, J. H.; Peterson, A. A.; Nørskov, J. K. Insights into C-C Coupling in CO2 Electroreduction on Copper Electrodes. ChemCatChem 2013, 5, 737-742.

(282) Sandberg, R. B.; Montoya, J. H.; Chan, K.; N??rskov, J. K.; Nørskov, J. K. CO-CO Coupling on Cu Facets: Coverage, Strain and Field Effects. Surf. Sci. 2016, 654, 56-62.

(283) Jiang, K.; Sandberg, R. B.; Akey, A. J.; Liu, X.; Bell, D. C.; Nørskov, J. K.; Chan, K.; Wang, $\mathrm{H}$. Metal Ion Cycling of $\mathrm{Cu}$ Foil for Selective $\mathrm{C}-\mathrm{C}$ Coupling in Electrochemical CO2 Reduction. Nat. Catal. 2018, 1, 111-119.

(284) Calle-Vallejo, F.; Koper, M. T. M. Theoretical Considerations on the Electroreduction of CO to $\mathrm{C} 2$ Species on $\mathrm{Cu}(100)$ Electrodes. Angew. Chemie - Int. Ed. 2013, 52, 7282-7285.

(285) Lausche, A. C.; Medford, A. J.; Khan, T. S.; Xu, Y.; Bligaard, T.; Abild-Pedersen, F.; Nørskov, J. K.; Studt, F. On the Effect of Coverage-Dependent Adsorbate-adsorbate Interactions for CO Methanation on Transition Metal Surfaces. J. Catal. 2013, 307, 275-282.

(286) Roberts, F. S.; Kuhl, K. P.; Nilsson, A. Electroreduction of Carbon Monoxide over a Copper Nanocube Catalyst: Surface Structure and PH Dependence on Selectivity. ChemCatChem 2016, 8, 1119-1124.

(287) Singh, M. R.; Goodpaster, J. D.; Weber, A. Z.; Head-gordon, M.; Bell, A. T. Mechanistic Insights into Electrochemical Reduction of $\mathrm{CO} 2$ over Ag Using Density Functional Theory and Transport Models. Proc. Natl. Acad. Sci. 2017, 114, E8812-E8821.

(288) Feaster, J. T.; Shi, C.; Cave, E. R.; Hatsukade, T.; Abram, D. N.; Kuhl, K. P.; Hahn, C.; Nørskov, J. K.; Jaramillo, T. F. Understanding Selectivity for the Electrochemical Reduction of Carbon Dioxide to Formic Acid and Carbon Monoxide on Metal Electrodes. ACS Catal. 2017, 7, 4822-4827.

(289) Schmid, B.; Reller, C.; Neubauer, S. S.; Fleischer, M.; Dorta, R.; Schmid, G. Reactivity of Copper Electrodes towards Functional Groups and Small Molecules in the Context of CO2 Electro-Reductions. Catalysts 2017, 7, 161.

(290) Ren, D.; Fong, J.; Yeo, B. S. The Effects of Currents and Potentials on the Selectivities of Copper toward Carbon Dioxide Electroreduction. Nat. Commun. 2018, 9, 925.

(291) Scott, S. B. Investigating the Electrochemical Reduction of Carbon Dioxide Using In Situ Mass Spectrometry, Technical University of Denmark, 2016.

(292) Akhade, S. A.; Luo, W.; Nie, X.; Asthagiri, A.; Janik, M. J. Theoretical Insight on Reactivity Trends in CO2 Electroreduction across Transition Metals. Catal. Sci. Technol. 2016, 6, 10421053.

(293) Ma, S.; Sadakiyo, M.; Luo, R.; Heima, M.; Yamauchi, M.; Kenis, P. J. A. One-Step Electrosynthesis of Ethylene and Ethanol from $\mathrm{CO} 2$ in an Alkaline Electrolyzer. J. Power Sources 2016, 301, 219-228.

(294) Handoko, A. D.; Chan, K. W.; Yeo, B. S. -CH3 Mediated Pathway for the Electroreduction of $\mathrm{CO} 2$ to Ethane and Ethanol on Thick Oxide-Derived Copper Catalysts at Low Overpotentials. ACS Energy Lett. 2017, 2, 2103-2109.

(295) Smith, B. D.; Irish, D. E.; Kedzierzawski, P.; Augustynski, J. A Surface Enhanced Roman Scattering Study of the Intermediate and Poisoning Species Formed during the Electrochemical Reduction of CO2 on Copper. J. Electrochem. Soc. 1997, 144, 4288-4296.

(296) Mistry, H.; Varela, A. S.; Kühl, S.; Strasser, P.; Cuenya, B. R. Nanostructured Electrocatalysts with Tunable Activity and Selectivity. Nat. Rev. Mater. 2016, 1, 16009. 
(297) Lu, Q.; Rosen, J.; Jiao, F. Nanostructured Metallic Electrocatalysts for Carbon Dioxide Reduction. ChemCatChem 2015, 7, 38-47.

(298) Vesborg, P. C. K.; Jaramillo, T. F. Addressing the Terawatt Challenge: Scalability in the Supply of Chemical Elements for Renewable Energy. RSC Adv. 2012, 2, 7933-7947.

(299) Reske, R.; Mistry, H.; Behafarid, F.; Roldan Cuenya, B.; Strasser, P. Particle Size Effects in the Catalytic Electroreduction of $\mathrm{CO} 2$ on Cu Nanoparticles. J. Am. Chem. Soc. 2014, 136, 6978-6986.

(300) Manthiram, K.; Beberwyck, B. J.; Alivisatos, A. P. Enhanced Electrochemical Methanation of Carbon Dioxide with a Dispersible Nanoscale Copper Catalyst. J. Am. Chem. Soc. 2014, 136, 13319-13325.

(301) Loiudice, A.; Lobaccaro, P.; Kamali, E. A.; Thao, T.; Huang, B. H.; Ager, J. W.; Buonsanti, R. Tailoring Copper Nanocrystals towards $\mathrm{C} 2$ Products in Electrochemical CO2 Reduction. Angew. Chemie Int. Ed. 2016, 55, 5789-5792.

(302) Mistry, H.; Behafarid, F.; Reske, R.; Varela, A. S.; Strasser, P.; Roldan Cuenya, B. Tuning Catalytic Selectivity at the Mesoscale via Interparticle Interactions. ACS Catal. 2016, 6, 10751080.

(303) Kim, D.; Kley, C. S.; Li, Y.; Yang, P. Copper Nanoparticle Ensembles for Selective Electroreduction of CO2 to C2-C3 Products. Proc. Natl. Acad. Sci. 2017, 114, 10560-10565.

(304) Li, Y.; Cui, F.; Ross, M. B.; Kim, D.; Sun, Y.; Yang, P. Structure-Sensitive CO2 Electroreduction to Hydrocarbons on Ultrathin Five-Fold Twinned Copper Nanowires. Nano Lett. 2017, 17, 1312-1317.

(305) Wang, Z.; Yang, G.; Zhang, Z.; Jin, M.; Yin, Y. Selectivity on Etching: Creation of HighEnergy Facets on Copper Nanocrystals for CO2 Electrochemical Reduction. ACS Nano 2016, $10,4559-4564$.

(306) Baturina, O. A.; Lu, Q.; Padilla, M. A.; Xin, L.; Li, W.; Serov, A.; Artyushkova, K.; Atanassov, P.; Xu, F.; Epshteyn, A.; et al. CO2 Electroreduction to Hydrocarbons on CarbonSupported Cu Nanoparticles. ACS Catal. 2014, 4, 3682-3695.

(307) Gupta, K.; Bersani, M.; Darr, J. A. Highly Efficient Electro-Reduction of CO2 to Formic Acid by Nano-Copper. J. Mater. Chem. A 2016, 4, 13786-13794.

(308) Stephens, I. E. L.; Bondarenko, A. S.; Grønbjerg, U.; Rossmeisl, J.; Chorkendorff, I. Understanding the Electrocatalysis of Oxygen Reduction on Platinum and Its Alloys. Energy Environ. Sci. 2012, 5, 6744-6762.

(309) Hammer, B.; Morikawa, Y.; Nørskov, J. K. CO Chemisorption at Metal Surfaces and Overlayers. Phys. Rev. Lett. 1996, 76, 2141-2144.

(310) Grosse, P.; Gao, D.; Scholten, F.; Sinev, I.; Mistry, H.; Roldan Cuenya, B. Dynamic Changes in the Structure, Chemical State and Catalytic Selectivity of Cu Nanocubes during CO2 Electroreduction: Size and Support Effects. Angew. Chemie Int. Ed. 2018, 57, 6192-6197.

(311) Waugh, K. C. Methanol Synthesis. Catal. Today 1992, 15, 51-75.

(312) Song, Y.; Peng, R.; Hensley, D. K.; Bonnesen, P. V.; Liang, L.; Wu, Z.; Meyer, H. M.; Chi, M.; Ma, C.; Sumpter, B. G.; et al. High-Selectivity Electrochemical Conversion of $\mathrm{CO} 2$ to Ethanol Using a Copper Nanoparticle/N-Doped Graphene Electrode. ChemistrySelect 2016, 1, $1-8$.

(313) Yuan, J.; Zhang, J.-J.; Yang, M.-P.; Meng, W.-J.; Wang, H.; Lu, J.-X. CuO Nanoparticles Supported on $\mathrm{TiO} 2$ with High Efficiency for $\mathrm{CO} 2$ Electrochemical Reduction to Ethanol. Catalysts 2018, 8, 171. 
(314) Huo, Y.; Peng, X.; Liu, X.; Li, H.; Luo, J. High Selectivity Toward C2H4 Production over Cu Particles Supported by Butterfly-Wing-Derived Carbon Frameworks. ACS Appl. Mater.

Interfaces 2018, 10, 12618-12625.

(315) Yang, H.-J.; Yang, H.; Hong, Y.-H.; Zhang, P.-Y.; Wang, T.; Chen, L.-N.; Zhang, F.-Y.; Wu, Q.-H.; Tian, N.; Zhou, Z.-Y.; et al. Promoting Ethylene Selectivity from CO2 Electroreduction on $\mathrm{CuO}$ Supported onto CO2 Capture Materials. ChemSusChem 2018, 11, 881-887.

(316) Feng, X.; Jiang, K.; Fan, S.; Kanan, M. W. A Direct Grain-Boundary-Activity Correlation for CO Electroreduction on Cu Nanoparticles. ACS Cent. Sci. 2016, 2, 169-174.

(317) Gao, D.; Zhang, Y.; Zhou, Z.; Cai, F.; Zhao, X.; Huang, W.; Li, Y.; Zhu, J.; Liu, P.; Yang, F.; et al. Enhancing CO2 Electroreduction with the Metal-Oxide Interface. J. Am. Chem. Soc. 2017, 139, 5652-5655.

(318) Rogers, C.; Perkins, W. S.; Veber, G.; Williams, T. E.; Cloke, R. R.; Fischer, F. R. Synergistic Enhancement of Electrocatalytic $\mathrm{CO} 2$ Reduction with Gold Nanoparticles Embedded in Functional Graphene Nanoribbon Composite Electrodes. J. Am. Chem. Soc. 2017, 139, 40524061.

(319) Velázquez, A.; Centellas, F.; Garrido, J. A.; Arias, C.; Rodríguez, R. M.; Brillas, E.; Cabot, P. L. Structure of Carbon-Supported Pt-Ru Nanoparticles and Their Electrocatalytic Behavior for Hydrogen Oxidation Reaction. J. Power Sources 2010, 195, 710-719.

(320) Frese, K. W. Electrochemical Reduction of CO2 at Intentionally Oxidized Copper Electrodes. J. Electrochem. Soc. 1991, 138, 3338-3340.

(321) Ma, M.; Djanashvili, K.; Smith, W. A. Controllable Hydrocarbon Formation from the Electrochemical Reduction of $\mathrm{CO} 2$ over $\mathrm{Cu}$ Nanowire Arrays. Angew. Chemie Int. Ed. 2016, $55,6680-6684$.

(322) Rahaman, M.; Dutta, A.; Zanetti, A.; Broekmann, P. Electrochemical Reduction of CO2 into Multicarbon Alcohols on Activated Cu Mesh Catalysts: An Identical Location (IL) Study. ACS Catal. 2017, 7, 7946-7956.

(323) Raciti, D.; Cao, L.; Livi, K. J. T.; Rottmann, P. F.; Tang, X.; Li, C.; Hicks, Z.; Bowen, K. H.; Hemker, K. J.; Mueller, T.; et al. Low-Overpotential Electroreduction of Carbon Monoxide Using Copper Nanowires. ACS Catal. 2017, 7, 4467-4472.

(324) Haynes, W. M. Handbook of Chemistry and Physics, 96th ed.; Haynes, W. M., Bruno, T. J., Lide, D. R., Eds.; CRC Press: Boca Raton, FL, 2015.

(325) Verdaguer-Casadevall, A.; Li, C. W.; Johansson, T. P.; Scott, S. B.; McKeown, J. T.; Kumar, M.; Stephens, I. E. L.; Kanan, M. W.; Chorkendorff, I. Probing the Active Surface Sites for CO Reduction on Oxide-Derived Copper Electrocatalysts. J. Am. Chem. Soc. 2015, 137, 98089811.

(326) Wang, Y.; Raciti, D.; Wang, C. High-Flux CO Reduction Enabled by Three-Dimensional Nanostructured Copper Electrodes. ACS Catal. 2018, 8, 5657-5663.

(327) Tang, W.; Peterson, A. A.; Varela, A. S.; Jovanov, Z. P.; Bech, L.; Durand, W. J.; Dahl, S.; Nørskov, J. K.; Chorkendorff, I. The Importance of Surface Morphology in Controlling the Selectivity of Polycrystalline Copper for CO2 Electroreduction. Phys. Chem. Chem. Phys. 2012, 14, 76-81.

(328) Ren, D.; Wong, N. T.; Handoko, A. D.; Huang, Y.; Yeo, B. S. Mechanistic Insights into the Enhanced Activity and Stability of Agglomerated Cu Nanocrystals for the Electrochemical Reduction of Carbon Dioxide to N-Propanol. J. Phys. Chem. Lett. 2016, 7, 20-24.

(329) Gao, D.; Zegkinoglou, I.; Divins, N. J.; Scholten, F.; Sinev, I.; Grosse, P.; Roldan Cuenya, B. 
Plasma-Activated Copper Nanocube Catalysts for Efficient Carbon Dioxide Electroreduction to Hydrocarbons and Alcohols. ACS Nano 2017, 11, 4825-4831.

(330) Kim, D.; Lee, S.; Ocon, J. D.; Jeong, B.; Lee, J. K.; Lee, J. Insights into Autonomously Formed Oxygen-Evacuated Cu2O Electrode for the Selective Production of C2H4 from CO2. Phys. Chem. Chem. Phys. 2015, 17, 824-830.

(331) Reller, C.; Krause, R.; Volkova, E.; Schmid, B.; Neubauer, S.; Rucki, A.; Schuster, M.; Schmid, G. Selective Electroreduction of CO2 toward Ethylene on Nano Dendritic Copper Catalysts at High Current Density. Adv. Energy Mater. 2017, 7, 1602114.

(332) Yang, K. D.; Ko, W. R.; Lee, J. H.; Kim, S. J.; Lee, H.; Lee, M. H.; Nam, K. T. MorphologyDirected Selective Production of Ethylene or Ethane from $\mathrm{CO} 2$ on a $\mathrm{Cu}$ Mesopore Electrode. Angew. Chemie Int. Ed. 2016, 56, 796-800.

(333) Dutta, A.; Rahaman, M.; Luedi, N. C.; Mohos, M.; Broekmann, P. Morphology Matters: Tuning the Product Distribution of $\mathrm{CO} 2$ Electroreduction on Oxide-Derived Cu Foam Catalysts. ACS Catal. 2016, 6, 3804-3814.

(334) Dutta, A.; Rahaman, M.; Mohos, M.; Zanetti, A.; Broekmann, P. Electrochemical CO2 Conversion Using Skeleton (Sponge) Type of Cu Catalysts. ACS Catal. 2017, 7, 5431-5437.

(335) Gonçalves, M. R.; Gomes, A.; Condeço, J.; Fernandes, T. R. C.; Pardal, T.; Sequeira, C. A. C.; Branco, J. B. Electrochemical Conversion of CO2 to C2 Hydrocarbons Using Different Ex Situ Copper Electrodeposits. Electrochim. Acta 2013, 102, 388-392.

(336) Peng, Y.; Wu, T.; Sun, L.; Nsanzimana, J. M. V.; Fisher, A. C.; Wang, X. Selective Electrochemical Reduction of CO2 to Ethylene on Nanopores Modified Copper Electrodes in Aqueous Solution. ACS Appl. Mater. Interfaces 2017, 9, 32782-32789.

(337) Song, H.; Im, M.; Song, J. T.; Lim, J. A.; Kim, B. S.; Kwon, Y.; Ryu, S.; Oh, J. Effect of Mass Transfer and Kinetics in Ordered Cu-Mesostructures for Electrochemical CO2 Reduction. Appl. Catal. B Environ. 2018, 232, 391-396.

(338) Favaro, M.; Xiao, H.; Cheng, T.; Goddard, W. A.; Yano, J.; Crumlin, E. J. Subsurface Oxide Plays a Critical Role in $\mathrm{CO} 2$ Activation by $\mathrm{Cu}(111)$ Surfaces to Form Chemisorbed CO2, the First Step in Reduction of CO2. Proc. Natl. Acad. Sci. 2017, 114, 6706-6711.

(339) Liu, C.; Lourenço, M. P.; Hedström, S.; Cavalca, F.; Diaz-Morales, O.; Duarte, H. A.; Nilsson, A.; Pettersson, L. G. M. Stability and Effects of Subsurface Oxygen in Oxide-Derived $\mathrm{Cu}$ Catalyst for CO2 Reduction. J. Phys. Chem. C 2017, 121, 25010-25017.

(340) Garza, A.; Bell, A. T.; Head-Gordon, M. Is Subsurface Oxygen Necessary for the Electrochemical Reduction of CO 2 on Copper? J. Phys. Chem. Lett. 2018, 9, 601-606.

(341) Fields, M.; Hong, X.; Nørskov, J. K.; Chan, K. Role of Subsurface Oxygen on Cu Surfaces for CO2 Electrochemical Reduction. J. Phys. Chem. C 2018, 122, 16209-16215.

(342) Terunuma, Y.; Saitoh, A.; Momose, Y. Relationship between Hydrocarbon Production in the Electrochemical Reduction of $\mathrm{CO} 2$ and the Characteristics of the Cu Electrode. J. Electroanal. Chem. 1997, 434, 69-75.

(343) Le, M.; Ren, M.; Zhang, Z.; Sprunger, P. T.; Kurtz, R. L.; Flake, J. C. Electrochemical Reduction of $\mathrm{CO} 2$ to $\mathrm{CH} 3 \mathrm{OH}$ at Copper Oxide Surfaces. J. Electrochem. Soc. 2011, 158, E45E49.

(344) Cavalca, F.; Ferragut, R.; Aghion, S.; Eilert, A.; Diaz-Morales, O.; Liu, C.; Koh, A. L.; Hansen, T. W.; Pettersson, L. G. M.; Nilsson, A. Nature and Distribution of Stable Subsurface Oxygen in Copper Electrodes During Electrochemical CO2 Reduction. J. Phys. Chem. C 2017, $121,25003-25009$. 
(345) Lee, S. Y.; Jung, H.; Kim, N.-K.; Oh, H.-S.; Min, B. K.; Hwang, Y. J. Mixed Copper States in Anodized $\mathrm{Cu}$ Electrocatalyst for Stable and Selective Ethylene Production from CO2 Reduction. J. Am. Chem. Soc. 2018, 140, 8681-8689.

(346) Platzman, I.; Brener, R.; Haick, H.; Tannenbaum, R. Oxidation of Polycrystalline Copper Thin Films at Ambient Conditions. J. Phys. Chem. C 2008, 112, 1101-1108.

(347) Lum, Y.; Ager, J. W. Stability of Residual Oxides in Oxide-Derived Copper Catalysts for Electrochemical CO2 Reduction Investigated with 180 Labeling. Angew. Chemie Int. Ed. 2018, 57, 551-554.

(348) Zhuang, T.-T.; Liang, Z.-Q.; Seifitokaldani, A.; Li, Y.; De Luna, P.; Burdyny, T.; Che, F.; Meng, F.; Min, Y.; Quintero-Bermudez, R.; et al. Steering Post-C-C Coupling Selectivity Enables High Efficiency Electroreduction of Carbon Dioxide to Multi-Carbon Alcohols. Nat. Catal. 2018, 1, 421-428.

(349) Zhou, Y.; Che, F.; Liu, M.; Zou, C.; Liang, Z.; De Luna, P.; Yuan, H.; Li, J.; Wang, Z.; Xie, H.; et al. Dopant-Induced Electron Localization Drives CO2 Reduction to C2 Hydrocarbons. Nat. Chem. 2018, 10, 974-980.

(350) Eilert, A.; Cavalca, F.; Roberts, F. S.; Osterwalder, J.; Liu, C.; Favaro, M.; Crumlin, E. J.; Ogasawara, H.; Friebel, D.; Pettersson, L. G. M.; et al. Subsurface Oxygen in Oxide-Derived Copper Electrocatalysts for Carbon Dioxide Reduction. J. Phys. Chem. Lett. 2017, 8, 285-290.

(351) Kas, R.; Hummadi, K. K.; Kortlever, R.; de Wit, P.; Milbrat, A.; Luiten-Olieman, M. W. J.; Benes, N. E.; Koper, M. T. M.; Mul, G. Three-Dimensional Porous Hollow Fibre Copper Electrodes for Efficient and High-Rate Electrochemical Carbon Dioxide Reduction. Nat. Commun. 2016, 7, 10748.

(352) Dinh, C.; Burdyny, T.; Kibria, M. G.; Seifitokaldani, A.; Gabardo, C. M.; García de Arquer, F. P.; Kiani, A.; Edwards, J. P.; De Luna, P.; Bushuyev, O. S.; et al. CO2 Electroreduction to Ethylene via Hydroxide-Mediated Copper Catalysis at an Abrupt Interface. Science 2018, 360, 783-787.

(353) Lv, J.; Jouny, M.; Luc, W.; Zhu, W.; Zhu, J.; Jiao, F. A Highly Porous Copper Electrocatalyst for Carbon Dioxide Reduction. Adv. Mater. 2018, 30, 1803111.

(354) Han, L.; Zhou, W.; Xiang, C. High-Rate Electrochemical Reduction of Carbon Monoxide to Ethylene Using Cu-Nanoparticle-Based Gas Diffusion Electrodes. ACS Energy Lett. 2018, 3, 855-860.

(355) Higgins, D.; Hahn, C.; Xiang, C.; Jaramillo, T. F.; Weber, A. Z. Gas-Diffusion Electrodes for Carbon Dioxide Reduction: A New Paradigm. ACS Energy Lett. 2019, 4, 317-324.

(356) Weekes, D. M.; Salvatore, D. A.; Reyes, A.; Huang, A.; Berlinguette, C. P. Electrolytic CO2 Reduction in a Flow Cell. Acc. Chem. Res. 2018, 51, 910-918.

(357) Ponec, V. Alloy Catalysts: The Concepts. Appl. Catal. A Gen. 2001, 222, 31-45.

(358) Rodriguez, J. A. Physical and Chemical Properties of Bimetallic Surfaces. Surf. Sci. Rep. 1996, 24, 223-287.

(359) Sinfelt, J. H.; Carter, J. L.; Yates, D. J. C. Catalytic Hydrogenolysis and Dehydrogenation over Copper-Nickel Alloys. J. Catal. 1972, 24, 283-296.

(360) Kitchin, J. R.; Nørskov, J. K.; Barteau, M. A.; Chen, J. G. Role of Strain and Ligand Effects in the Modification of the Electronic and Chemical Properties of Bimetallic Surfaces. Phys. Rev. Lett. 2004, 93, 4-7.

(361) Mavrikakis, M.; Hammer, B.; Nørskov, J. K. Effect of Strain on the Reactivity of Metal Surfaces. Phys. Rev. Lett. 1998, 81, 2819-2822. 
(362) Gsell, M. Effect of Substrate Strain on Adsorption. Science 1998, 280, 717-720.

(363) Sachtler, W. M. H.; van der Plank, P. The Role of Individual Surface Atoms in Chemisorption and Catalysis by Nickel-Copper Alloys. Surf. Sci. 1969, 18, 62-79.

(364) Sachtler, J. W. A.; Somorjai, G. A. Influence of Ensemble Size on CO Chemisorption and Catalytic N-Hexane Conversion by Au-Pt(111) Bimetallic Single-Crystal Surfaces. J. Catal. 1983, 81, 77-94.

(365) Kitchin, J. R.; Nørskov, J. K.; Barteau, M. A.; Chen, J. G. Modification of the Surface Electronic and Chemical Properties of $\operatorname{Pt}(111)$ by Subsurface $3 \mathrm{~d}$ Transition Metals. J. Chem. Phys. 2004, 120, 10240-10246.

(366) Hansen, H. A.; Shi, C.; Lausche, A. C.; Peterson, A. A.; Nørskov, J. K. Bifunctional Alloys for the Electroreduction of CO2 and CO. Phys. Chem. Chem. Phys. 2016, 18, 9194-9201.

(367) M., K.; Tripkovic, V.; Rossmeisl, J. Intermetallic Alloys as CO Electroreduction Catalysts the Role of Isolated Active Sites. ACS Catal. 2014, 4, 2268-2273.

(368) Cheng, M. J.; Clark, E. L.; Pham, H. H.; Bell, A. T.; Head-Gordon, M. Quantum Mechanical Screening of Single-Atom Bimetallic Alloys for the Selective Reduction of CO2 to C1 Hydrocarbons. ACS Catal. 2016, 6, 7769-7777.

(369) Hirunsit, P. Electroreduction of Carbon Dioxide to Methane on Copper, Copper-Silver, and Copper-Gold Catalysts: A DFT Study. J. Phys. Chem. C 2013, 117, 8262-8268.

(370) Hirunsit, P.; Soodsawang, W.; Limtrakul, J. CO2 Electrochemical Reduction to Methane and Methanol on Copper-Based Alloys: Theoretical Insight. J. Phys. Chem. C 2015, 119, 82388249.

(371) Calle-Vallejo, F.; Koper, M. T. M. Accounting for Bifurcating Pathways in the Screening for CO2 Reduction Catalysts. ACS Catal. 2017, 7, 7346-7351.

(372) Hahn, C.; Abram, D. N.; Hansen, H. A.; Hatsukade, T.; Jackson, A.; Johnson, N. C.; Hellstern, T. R.; Kuhl, K. P.; Cave, E. R.; Feaster, J. T.; et al. Synthesis of Thin Film AuPd Alloys and Their Investigation for Electrocatalytic CO2 Reduction. J. Mater. Chem. A 2015, 3, 2018520194.

(373) Kortlever, R.; Peters, I.; Balemans, C.; Kas, R.; Kwon, Y.; Mul, G.; Koper, M. T. M. Palladium-gold Catalyst for the Electrochemical Reduction of $\mathrm{CO} 2$ to $\mathrm{C} 1-\mathrm{C} 5$ Hydrocarbons. Chem. Commun. 2016, 52, 10229-10232.

(374) Jovanov, Z. P.; Hansen, H. A.; Varela, A. S.; Malacrida, P.; Peterson, A. A.; Nørskov, J. K.; Stephens, I. E. L.; Chorkendorff, I. Opportunities and Challenges in the Electrocatalysis of $\mathrm{CO} 2$ and CO Reduction Using Bifunctional Surfaces: A Theoretical and Experimental Study of Au-Cd Alloys. J. Catal. 2016, 343, 215-231.

(375) Torelli, D. A.; Francis, S. A.; Crompton, J. C.; Javier, A.; Thompson, J. R.; Brunschwig, B. S.; Soriaga, M. P.; Lewis, N. S. Nickel-Gallium-Catalyzed Electrochemical Reduction of CO2 to Highly Reduced Products at Low Overpotentials. ACS Catal. 2016, 6, 2100-2104.

(376) Paris, A. R.; Bocarsly, A. B. Ni-Al Films on Glassy Carbon Electrodes Generate an Array of Oxygenated Organics from CO2. ACS Catal. 2017, 7, 6815-6820.

(377) Sun, K.; Cheng, T.; Wu, L.; Hu, Y.; Zhou, J.; Maclennan, A.; Jiang, Z.; Gao, Y.; Goddard, W. A.; Wang, Z. Ultrahigh Mass Activity for Carbon Dioxide Reduction Enabled by Gold-Iron Core-Shell Nanoparticles. J. Am. Chem. Soc. 2017, 139, 15608-15611.

(378) Hori, Y.; Murata, A.; Ito, S.; Yoshinami, Y.; Koga, O. Nickel and Iron Modified Copper Electrode for Electroreduction of CO2 by In-Situ Electrodeposition. Chem. Lett. 1989, 18, $1567-1570$. 
(379) Todoroki, N.; Yokota, N.; Nakahata, S.; Nakamura, H.; Wadayama, T. Electrochemical Reduction of $\mathrm{CO} 2$ on Ni- and Pt-Epitaxially Grown Cu(111) Surfaces. Electrocatalysis 2016, 7, 97-103.

(380) Zhao, X.; Luo, B.; Long, R.; Wang, C.; Xiong, Y. Composition-Dependent Activity of Cu-Pt Alloy Nanocubes for Electrocatalytic CO2 Reduction. J. Mater. Chem. A 2015, 3, 4134-4138.

(381) Guo, X.; Zhang, Y.; Deng, C.; Li, X.; Xue, Y.; Yan, Y.-M.; Sun, K. Composition Dependent Activity of $\mathrm{Cu}-\mathrm{Pt}$ Nanocrystals for Electrochemical Reduction of CO2. Chem. Commun. 2015, $51,1345-1348$.

(382) Varela, A. S.; Schlaup, C.; Jovanov, Z. P.; Malacrida, P.; Horch, S.; Stephens, I. E. L.; Chorkendorff, I. CO2 Electroreduction on Well-Defined Bimetallic Surfaces: Cu Overlayers on $\operatorname{Pt}(111)$ and $\operatorname{Pt}(211)$. J. Phys. Chem. C 2013, 117, 20500-20508.

(383) Reske, R.; Duca, M.; Oezaslan, M.; Schouten, K. J. P.; Koper, M. T. M.; Strasser, P. Controlling Catalytic Selectivities during CO2 Electroreduction on Thin Cu Metal Overlayers. J. Phys. Chem. Lett. 2013, 4, 2410-2413.

(384) Watanabe, M.; Shibata, M.; Kato, A. Design of Alloy Electrocatalysts for CO2 Reduction: Improved Energy Efficiency, Selectivity, and Reaction Rate for the CO2 Electroreduction on $\mathrm{Cu}$ Alloy Electrodes. J. Electroanal. Chem. Interfacial Electrochem. 1991, 305, 319-328.

(385) Watanabe, M. Design of Alloy Electrocatalysts for CO2 Reduction: III. The Selective and Reversible Reduction of CO2 on Cu Alloy Electrodes. J. Electrochem. Soc. 1991, 138, 3382 3389.

(386) Yoshitake, H.; Takahashi, K.; Ota, K. Electrochemical Reduction of CO2 on HydrogenEnriched and Hydrogen-Depleted Surfaces. J. Chem. Soc. Trans. 1994, 90, 155-159.

(387) Ohkawa, K.; Noguchi, Y.; Nakayama, S.; Hashimoto, K.; Fujishima, A. Electrochemical Reduction of Carbon Dioxide on Hydrogen-Storing Materials. Part II. Copper-Modified Palladium Electrode. J. Electroanal. Chem. 1993, 348, 459-464.

(388) Ohkawa, K.; Noguchi, Y.; Nakayama, S.; Hashimoto, K.; Fujishima, A. Electrochemical Reduction of Carbon Dioxide on Hydrogen-Storing Materials. Part 3. The Effect of the Absorption of Hydrogen on the Palladium Electrodes Modified with Copper. J. Electroanal. Chem. 1994, 367, 165-173.

(389) Chen, C. S.; Wan, J. H.; Yeo, B. S. Electrochemical Reduction of Carbon Dioxide to Ethane Using Nanostructured Cu2O-Derived Copper Catalyst and Palladium(II) Chloride. J. Phys. Chem. C 2015, 119, 26875-26882.

(390) Yin, Z.; Gao, D.; Yao, S.; Zhao, B.; Cai, F.; Lin, L.; Tang, P.; Zhai, P.; Wang, G.; Ma, D.; et al. Highly Selective Palladium-Copper Bimetallic Electrocatalysts for the Electrochemical Reduction of CO2 to CO. Nano Energy 2016, 27, 35-43.

(391) Li, M.; Wang, J. J.; Li, P.; Chang, K.; Li, C. L.; Wang, T.; Jiang, B.; Zhang, H. B.; Liu, H. M.; Yamauchi, Y.; et al. Mesoporous Palladium-Copper Bimetallic Electrodes for Selective Electrocatalytic Reduction of Aqueous CO2 to CO. J. Mater. Chem. A 2016, 4, 4776-4782.

(392) Rochefort, A.; Abon, M.; Delichère, P.; Bertolini, J. C. Alloying Effect on the Adsorption Properties of Pd50Cu50\{111\} Single Crystal Surface. Surf. Sci. Lett. 1993, 294, 43-52.

(393) Ma, S.; Sadakiyo, M.; Heima, M.; Luo, R.; Haasch, R. T.; Gold, J. I.; Yamauchi, M.; Kenis, P. J. A. Electroreduction of Carbon Dioxide to Hydrocarbons Using Bimetallic Cu-Pd Catalysts with Different Mixing Patterns. J. Am. Chem. Soc. 2017, 139, 47-50.

(394) Choi, J.; Kim, M. J.; Ahn, S. H.; Choi, I.; Jang, J. H.; Ham, Y. S.; Kim, J. J.; Kim, S. K. Electrochemical CO2 Reduction to CO on Dendritic Ag-Cu Electrocatalysts Prepared by 
Electrodeposition. Chem. Eng. J. 2016, 299, 37-44.

(395) Kyriacou, G.; Anagnostopoulos, A. Electrochemical Reduction of $\mathrm{CO} 2$ at $\mathrm{Cu}+\mathrm{Au}$ Electrodes. J. Electroanal. Chem. 1992, 328, 233-243.

(396) Roy, C.; Galipaud, J.; Fréchette-Viens, L.; Garbarino, S.; Qiao, J.; Guay, D. CO2 Electroreduction at AuxCu1-x Obtained by Pulsed Laser Deposition in O2 Atmosphere. Electrochim. Acta 2017, 246, 115-122.

(397) Kim, D.; Resasco, J.; Yu, Y.; Asiri, A. M.; Yang, P. Synergistic Geometric and Electronic Effects for Electrochemical Reduction of Carbon Dioxide Using Gold-Copper Bimetallic Nanoparticles. Nat. Commun. 2014, 5, 4948.

(398) Kim, D.; Xie, C.; Becknell, N.; Yu, Y.; Karamad, M.; Chan, K.; Crumlin, E. J.; Nørskov, J. K.; Yang, P. Electrochemical Activation of CO2 through Atomic Ordering Transformations of AuCu Nanoparticles. J. Am. Chem. Soc. 2017, 139, 8329-8336.

(399) Jia, F.; Yu, X.; Zhang, L. Enhanced Selectivity for the Electrochemical Reduction of CO2 to Alcohols in Aqueous Solution with Nanostructured $\mathrm{Cu}-\mathrm{Au}$ Alloy as Catalyst. J. Power Sources 2014, 252, 85-89.

(400) Morales-Guio, C. G.; Cave, E. R.; Nitopi, S. A.; Feaster, J. T.; Wang, L.; Kuhl, K. P.; Jackson, A.; Johnson, N. C.; Abram, D. N.; Hatsukade, T.; et al. Improved CO2 Reduction Activity towards C2+ Alcohols on a Tandem Au on Cu Electrocatalyst. Nat. Catal. 2018, 1, 764-771.

(401) Lum, Y.; Ager, J. W. Sequential Catalysis Controls Selectivity in Electrochemical CO2 Reduction on Cu. Energy Environ. Sci. 2018, 11, 2935-2944.

(402) Higgins, D.; Landers, A. T.; Ji, Y.; Nitopi, S.; Morales-Guio, C. G.; Wang, L.; Chan, K.; Hahn, C.; Jaramillo, T. F. Guiding Electrochemical Carbon Dioxide Reduction toward Carbonyls Using Copper Silver Thin Films with Interphase Miscibility. ACS Energy Lett. 2018, 3, 2947-2955.

(403) Wang, Y.; Wang, D.; Dares, C. J.; Marquard, S. L.; Sheridan, M. V.; Meyer, T. J. CO2 Reduction to Acetate in Mixtures of Ultrasmall $(\mathrm{Cu}) \mathrm{n},(\mathrm{Ag}) \mathrm{m}$ Bimetallic Nanoparticles. Proc. Natl. Acad. Sci. 2017, 115, 278-283.

(404) Monzó, J.; Malewski, Y.; Kortlever, R.; Vidal-Iglesias, F. J.; Solla-Gullón, J.; Koper, M. T. M.; Rodriguez, P. Enhanced Electrocatalytic Activity of Au@Cu Core@ shell Nanoparticles towards CO2 Reduction. J. Mater. Chem. A 2015, 3, 23690-23698.

(405) Schlaup, C.; Horch, S.; Chorkendorff, I. On the Stability of Copper Overlayers on Au(111) and $\mathrm{Au}(100)$ Electrodes under Low Potential Conditions and in the Presence on CO and CO2. Surf. Sci. 2015, 631, 155-164.

(406) Friebel, D.; Mbuga, F.; Rajasekaran, S.; Miller, D. J.; Ogasawara, H.; Alonso-Mori, R.; Sokaras, D.; Nordlund, D.; Weng, T. C.; Nilsson, A. Structure, Redox Chemistry, and Interfacial Alloy Formation in Monolayer and Multilayer $\mathrm{Cu} / \mathrm{Au}(111)$ Model Catalysts for CO2 Electroreduction. J. Phys. Chem. C 2014, 118, 7954-7961.

(407) Detweiler, Z. M.; White, J. L.; Bernasek, S. L.; Bocarsly, A. B. Anodized Indium Metal Electrodes for Enhanced Carbon Dioxide Reduction in Aqueous Electrolyte. Langmuir 2014, $30,7593-7600$.

(408) Li, Q.; Fu, J.; Zhu, W.; Chen, Z.; Shen, B.; Wu, L.; Xi, Z.; Wang, T.; Lu, G.; Zhu, J.; et al. Tuning Sn-Catalysis for Electrochemical Reduction of $\mathrm{CO} 2$ to $\mathrm{CO}$ via the Core/Shell Cu/SnO2 Structure. J. Am. Chem. Soc. 2017, 139, 4290-4293.

(409) Hoffman, Z. B.; Gray, T. S.; Moraveck, K. B.; Gunnoe, T. B.; Zangari, G. The Electrochemical Reduction of Carbon Dioxide to Syngas and Formate at Dendritic Copper- 
Indium Electrocatalysts. ACS Catal. 2017, 7, 5381-5390.

(410) Larrazábal, G. O.; Shinagawa, T.; Martín, A. J.; Pérez-Ramírez, J. Microfabricated Electrodes Unravel the Role of Interfaces in Multicomponent Copper-Based CO2 Reduction Catalysts. Nat. Commun. 2018, 9, 1477.

(411) Luo, W.; Xie, W.; Mutschler, R.; Oveisi, E.; De Gregorio, G. L.; Buonsanti, R.; Züttel, A. Selective and Stable Electroreduction of $\mathrm{CO} 2$ to $\mathrm{CO}$ at the Copper/Indium Interface. ACS Catal. 2018, 8, 6571-6581.

(412) Zhao, Y.; Wang, C.; Wallace, G. G. Tin Nanoparticles Decorated Copper Oxide Nanowires for Selective Electrochemical Reduction of Aqueous CO2 to CO. J. Mater. Chem. A 2016, 4, 10710-10718.

(413) Sarfraz, S.; Garcia-Esparza, A. T.; Jedidi, A.; Cavallo, L.; Takanabe, K. Cu-Sn Bimetallic Catalyst for Selective Aqueous Electroreduction of CO2 to CO. ACS Catal. 2016, 6, 28422851.

(414) Rasul, S.; Anjum, D. H.; Jedidi, A.; Minenkov, Y.; Cavallo, L.; Takanabe, K. A Highly Selective Copper-Indium Bimetallic Electrocatalyst for the Electrochemical Reduction of Aqueous CO2 to CO. Angew. Chemie Int. Ed. 2015, 54, 2146-2150.

(415) Jedidi, A.; Rasul, S.; Masih, D.; Cavallo, L.; Takanabe, K. Generation of Cu-In Alloy Surfaces from CuInO 2 as Selective Catalytic Sites for CO 2 Electroreduction. J. Mater. Chem. A 2015, 3, 19085-19092.

(416) Larrazábal, G. O.; Martín, A. J.; Mitchell, S.; Hauert, R.; Pérez-Ramírez, J. Enhanced Reduction of $\mathrm{CO} 2$ to $\mathrm{CO}$ over $\mathrm{Cu}-\mathrm{In}$ Electrocatalysts: Catalyst Evolution Is the Key. ACS Catal. 2016, 6, 6265-6274.

(417) He, J.; Dettelbach, K. E.; Salvatore, D. A.; Li, T.; Berlinguette, C. P. High-Throughput Synthesis of Mixed-Metal Electrocatalysts for CO 2 Reduction. Angew. Chemie Int. Ed. 2017, $56,6068-6072$.

(418) Xie, H.; Chen, S.; Ma, F.; Liang, J.; Miao, Z.; Wang, T.; Wang, H.-L.; Huang, Y.; Li, Q. Boosting Tunable Syngas Formation via Electrochemical CO2 Reduction on $\mathrm{Cu} / \mathrm{In} 2 \mathrm{O} 3$ Core/Shell Nanoparticles. ACS Appl. Mater. Interfaces 2018, 10, 36996-37004.

(419) Zeng, J.; Bejtka, K.; Ju, W.; Castellino, M.; Chiodoni, A.; Sacco, A.; Farkhondehfal, M. A.; Hernández, S.; Rentsch, D.; Battaglia, C.; et al. Advanced Cu-Sn Foam for Selectively Converting CO2 to CO in Aqueous Solution. Appl. Catal. B Environ. 2018, 236, 475-482.

(420) Lv, W.; Zhou, J.; Kong, F.; Fang, H.; Wang, W. Porous Tin-Based Film Deposited on Copper Foil for Electrochemical Reduction of Carbon Dioxide to Formate. Int. J. Hydrogen Energy 2016, 41, 1585-1591.

(421) Wang, Y.; Zhou, J.; Lv, W.; Fang, H.; Wang, W. Electrochemical Reduction of CO2 to Formate Catalyzed by Electroplated Tin Coating on Copper Foam. Appl. Surf. Sci. 2016, 362, 394-398.

(422) Hori, Y.; Murata, A.; Kikuchi, K.; Suzuki, S. Electrochemical Reduction of Carbon Dioxides to Carbon Monoxide at a Gold Electrode in Aqueous Potassium Hydrogen Carbonate. $J$. Chem. Soc. Chem. Commun. 1987, 0, 728.

(423) Chen, Y.; Li, C. W.; Kanan, M. W. Aqueous CO2 Reduction at Very Low Overpotential on Oxide-Derived Au Nanoparticles. J. Am. Chem. Soc. 2012, 134, 19969-19972.

(424) Soriaga, M. P.; Baricuatro, J. H.; Javier, A. C.; Kim, Y.-G.; Cummins, K. D.; Tsang, C. F.; Hemminger, J. C.; Bui, N. N.; Stickney, J. L. Electrochemical Surface Science of CO2 Reduction at Well-Defined Cu Electrodes: Surface Characterization by Emersion, Ex Situ, In 
Situ, and Operando Methods; Elsevier Inc., 2017.

(425) Ulissi, Z. W.; Tang, M. T.; Xiao, J.; Liu, X.; Torelli, D. A.; Karamad, M.; Cummins, K.; Hahn, C.; Lewis, N. S.; Jaramillo, T. F.; et al. Machine-Learning Methods Enable Exhaustive Searches for Active Bimetallic Facets and Reveal New Active Site Motifs for CO2 Reduction. ACS Catal. 2017, 7, 6600-6608.

(426) Tran, K.; Ulissi, Z. W. Active Learning across Intermetallics to Guide Discovery of Electrocatalysts for CO2 Reduction and $\mathrm{H} 2$ Evolution. Nat. Catal. 2018, 1, 696-703.

(427) Ma, X.; Li, Z.; Achenie, L. E. K.; Xin, H. Machine-Learning-Augmented Chemisorption Model for CO2 Electroreduction Catalyst Screening. J. Phys. Chem. Lett. 2015, 6, 3528-3533.

(428) Endrődi, B.; Bencsik, G.; Darvas, F.; Jones, R.; Rajeshwar, K.; Janáky, C. Continuous-Flow Electroreduction of Carbon Dioxide. Prog. Energy Combust. Sci. 2017, 62, 133-154.

(429) Greenblatt, J. B.; Miller, D. J.; Ager, J. W.; Houle, F. A.; Sharp, I. D. The Technical and Energetic Challenges of Separating (Photo)Electrochemical Carbon Dioxide Reduction Products. Joule 2018, 2, 381-420.

(430) Duan, X.; Xu, J.; Wei, Z.; Ma, J.; Guo, S.; Wang, S.; Liu, H.; Dou, S. Metal-Free Carbon Materials for CO 2 Electrochemical Reduction. Adv. Mater. 2017, 29, 1701784. 


\section{Biographies}

Stephanie A. Nitopi was in the Schreyer Honors College at The Pennsylvania State University where she received her B.S. in Chemical Engineering in 2014. She is now a Ph.D. candidate in the Chemical Engineering department at Stanford University, and a member of Professor Tom Jaramillo's group and the SUNCAT Center for Interface Science and Catalysis. She was a recipient of a Stanford Graduate Fellowship in Science \& Engineering, and her current research is focused on the electrochemical conversion of carbon dioxide to fuels and chemicals on copper catalysts.

Erlend Bertheussen received his Bachelor's degree in Nanotechnology from the University of Bergen in 2012. He then moved to Denmark to do a Master's in Physics and Nanotechnology at the Technical University of Denmark (DTU), where his interest for electrochemical energy conversion started. He subsequently started a PhD degree in Physics at DTU, where he performed work within the field of CO reduction on copper electrodes. After graduating in the spring of 2018, he took a position as an R\&D Engineer in the start-up company RenCat ApS.

Soren B. Scott received a Bachelor's in Chemistry from the University of Copenhagen in 2013 before being introduced to the idea of $\mathrm{CO} 2$ electroreduction while working as a research assistant at the Joint Center for Artificial Photosynthesis at Lawrence Berkeley National Lab. He returned to Denmark for a Master's in Chemical Engineering at the Technical University of Denmark (DTU, 2016) and has since then been a Ph.D. student at DTU's Department of Physics. In 2018, Soren was awarded an EliteForsk travel grant from the Danish Ministry of Higher Education and Science. His current research uses insitu mass spectrometry to probe electrocatalytic interfaces.

Xinyan Liu received her B.S. in chemical engineering from Tsinghua University (Beijing, China) in 2013. She then obtained her Ph.D. in chemical engineering from Stanford University in 2018 under the supervision of Prof. Jens K. Nørskov, during which she has worked on density functional theory based microkinetic modeling of Fischer-Tropsch synthesis, electrochemical hydrogen evolution reaction, and 
electrochemical carbon monoxide/dioxide reduction reactions. Her expertise is in microkinetic modeling and data mining.

Albert K. Engstfeld (1983, Germany) graduated from the Ulm University in 2009 in chemistry with specialization in physical chemistry and surface science. He received his Ph.D. in 2015 from Ulm University on "Design and electrochemical / -catalytic properties of well defined bimetallic model catalyst electrodes," research conducted under the supervision of Prof. R.J. Behm. After a Post Doc position at the Institute for Surface Physics \& Catalysis (Technical University of Denmark), he returned in 2017 to Ulm University. He is currently working as a group leader (Nano-structured metal surfaces) at the Institute of Surface Chemistry and Catalysis. Combining an ultrahigh vacuum system with electrochemical methods he focusses on a fundamental understanding of restructuring/corrosion processes and electrocatalytic properties of nanostructured (bi)metallic single crystal model electrodes on an atomic scale level.

Sebastian Horch received his Ph.D. degree in Physics (1994) from Bonn University, Germany. He was Feodor Lynen Research Fellow at Arizona State University in 1995. Until 1999, he was Assistant Research Professor in the Center for Atomic-scale Materials Physics (CAMP) at Aarhus University, Denmark. Since 1999, he is Associate Professor at the Department of Physics, Denmarks Technical University (DTU). His main area of research is Surface Science using high resolution scanning probe microscopy.

Brian Seger completed his $\mathrm{PhD}$ in chemical engineering from Notre Dame under Prof. Prashant Kamat in 2009. After completing postdocs at the University of Queensland under Lian Zhou Wang and at DTU under Prof. Ib Chorkendorff, he became an assistant professor in the Surface Science and Catalysis section of DTU Physics in 2014 and then promoted to associated professor in 2017. He has published 45 peer reviewed papers that have been cited over 5,000 times. His research focuses on electrochemical $\mathrm{CO}_{2}$ reduction, photoelectrochemistry, semiconductor-electrolyte interfaces, and electro-synthesis reactions. 
Ifan E.L. Stephens was appointed as Senior Lecturer at the Department of Materials at Imperial College London in July 2017. Prior to Imperial, he was at the Department of Physics at the Technical University of Denmark (DTU); he was first employed as a postdoctoral researcher, then as assistant professor and finally as associate professor and leader of the Electrocatalysis Group there. In 2015, the Department of Mechanical Engineering at Massachusetts Institute of Technology (MIT) awarded Ifan the Peabody Visiting Associate Professorship. His team conducts model electrocatalysis experiments to enable the large-scale conversion of renewable energy to fuels and valuable chemicals and vice versa.

Karen Chan is an Associate Professor at the Department of Physics, Technical University of Denmark. Prior to this she was Associate Staff Scientist at SLAC National Accelerator Laboratory as part of the Joint Center for Artificial Photosynthesis. Her expertise is in the atomistic and continuum level modeling of electrochemical interfaces with applications in sustainable energy conversion. She obtained her PhD in 2013, advised by Michael Eikerling and held a postdoc fellowship from 20132016 at Stanford University, advised by Jens K. Nørskov.

Christopher Hahn completed his doctorate at the University of California Berkeley in 2012, where he studied bandgap engineering of 1-dimensional nitride and oxynitride materials for solar water splitting. After receiving his Ph.D., he conducted his postdoctoral research on catalyst discovery for electrochemical CO2 reduction at Stanford University within the SUNCAT Center for Interface Science and Catalysis. He began his current position at SLAC National Accelerator Laboratory in 2015, where he is conducting research with the Joint Center for Artificial Photosynthesis on catalyst discovery and understanding reaction mechanisms for electrochemical $\mathrm{CO} 2$ reduction.

Jens K. Nørskov is the Villum Kann Rasumussen professor at the Technical University of Denmark. His research aims at developing theoretical methods and concepts to understand and predict properties of materials. He is particularly interested in surface chemical properties, heterogeneous catalysis, electro-catalysis, and applications in energy conversion. 
Thomas Francisco Jaramillo is an Associate Professor of Chemical Engineering at Stanford University and of Photon Sciences at SLAC National Accelerator Laboratory, where he is the Director of the SUNCAT Center for Interface Science and Catalysis. A native of Puerto Rico, Prof. Jaramillo earned his B.S. in Chemical Engineering at Stanford University, followed by M.S. and Ph.D. in Chemical Engineering at the University of California at Santa Barbara (UCSB). Prof. Jaramillo conducted post-doctoral research in the Department of Physics at the Technical University of Denmark (DTU) as a Hans Christian Ørsted Post-doctoral Fellow. Prof. Jaramillo's research efforts are aimed at developing catalysts and processes that can accelerate important chemical transformations related to energy conversion with improved sustainability, efficiency, and durability. Prof. Jaramillo has earned a number of honors and awards for his efforts, including the Resonate Award from the Resnick Institute (2014), the Presidential Early Career Award for Scientists \& Engineers (PECASE, 2011), the U.S. Dept. of Energy Hydrogen and Fuel Cell Program Research \& Development Award (2011), the National Science Foundation (NSF) CAREER Award (2011), and the Mohr-Davidow Ventures (MDV) Innovator Award (2009).

Ib Chorkendorff is Professor in Heterogeneous Catalysis at DTU Physics. He earned his PhD in 1985 at Odense University Denmark, and after a post-doc at University of Pittsburgh, USA, he was employed in 1987 at DTU as Associate Professor for starting up the field of experimental surface science and catalysis. From 2005-2016 he was director of the Danish National Research Foundation Center for Individual Nanoparticle Functionality (CINF) and from 2016 he has been director of The Villum Center for the Science of Sustainable Fuels and Chemicals (V-SUSTAIN). Ib Chorkendorff's research activities focus on finding new catalysts for improving energy production/conversion and for environmental protection. He has author or coauthored more than 330 scientific papers, 18 patents and one textbook "Concepts of Modern Catalysis and Kinetics". He is co-founder of three start-up companies RENCAT APS, HPNOW APS and Spectroinlets APS. Ib Chorkendorff has received numerous prices and honors among others the Ulrik and Marie Brinch award and the Ingeborg and Leo 
Dannin's Prize for Natural Science Research. He is elected Fellow of The Academy for Technical Sciences (ATV) and Elected member of the Royal Danish Academy of Sciences and Letters.

\section{TOC Figure}

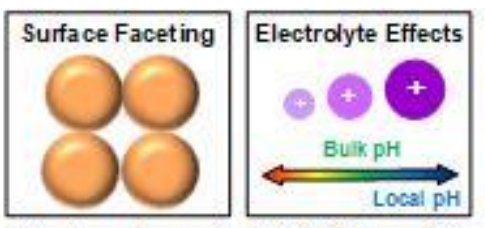

Electrochemical $\mathrm{CO}_{2} \mathrm{R}$ on $\mathrm{Cu}$

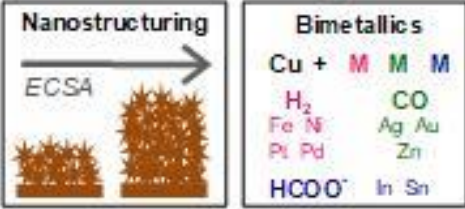

